\title{
Regulation and function of actin nucleators Dia and FMNL in the early Drosophila embryo
}

\author{
Dissertation \\ for the award of the degree \\ "Doctor rerum naturalium" (Dr.rer.nat.) \\ of the Georg-August Universität Göttingen \\ within the doctoral program "Biology" \\ submitted by \\ Anja Schmidt \\ from Bad Hersfeld, Germany
}

Göttingen, August 2018 


\section{Thesis Committee}

\section{Prof. Dr. J. Großhans}

Institute for Developmental Biochemistry, Medical School, Georg-August University Göttingen

Prof. Dr. R. Schuh

Department of Molecular Developmental Biology / Max-Planck-Institute for biophysical Chemistry, Göttingen

\section{Prof. Dr. R. Grosse}

Institute of Pharmacology / Philipps-University Marburg

\section{Members of the Examination Board}

Reviewer: Prof. Dr. J. Großhans

Institute for Developmental Biochemistry, Medical School, Georg-August University Göttingen

Second Reviewer: Prof. Dr. R. Schuh

Department of Molecular Developmental Biology / Max-Planck-Institute for biophysical Chemistry, Göttingen

Further members of the examination board:

\section{Prof. Dr. Gregor Bucher}

Evolutionary Developmental Genetics / Department of Developmental Biology, Georg-August University Göttingen

\section{Prof. Dr. Sigrid Hoyer-Fender}

Department of Developmental Biology / Georg-August University Göttingen

\section{Dr. Nico Posnien}

Department of Developmental Biology / Georg-August University Göttingen

\section{PD Dr. Gerd Vorbrüggen}

Department of Molecular Cell Dynamics / Max-Planck-Institute for biophysical Chemistry, Göttingen 


\section{Affidavit}

I hereby declare that I prepared the $\mathrm{PhD}$ thesis "Regulation and function of actin nucleators Dia and FMNL in the early Drosophila embryo" on my own with no other sources and aids than quoted.

Parts of this thesis were already published in Schmidt, A. and Grosshans, J. (2018). Dynamics of cortical domains in early Drosophila development. J Cell Sci 131, jcs212795 and Schmidt, A.*, Lv, Z.* and Großhans, J. (2018). ELMO and Sponge specify subapical restriction of Canoe and formation of the subapical domain in early Drosophila embryos. Development 145, dev157909 ( ${ }^{*}$ These authors contributed equally to this work).

The manuscript of the review Schmidt and Großhans (2018) was written by both authors.

The contributions to Schmidt et al., 2018 were as following: Dr. Zhiyi Lv mapped and characterized the mutant allele of ELMO and created the transgenic ELMO-GFP line. Furthermore, he did the crossings and imaging of Utrophin-GFP in wild type and ELMO background and the staining and imaging against Dlg, Slam and DNA of ELMO mutant embryos and prepared the figure. Both results are shown in Figure 15. The resulting experiments and figures shown in this paper were prepared by myself. The manuscript was written by Prof. J. Großhans and myself.

Dr. Shuling Yan contributed to Part B by performing early experiments on Dia and its role in domain formation. Henrik Steffen contributed to Part $C$ by the expression and purification of FMNL for the generation of antibodies. 


\section{Table of Contents}

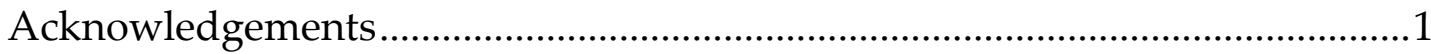

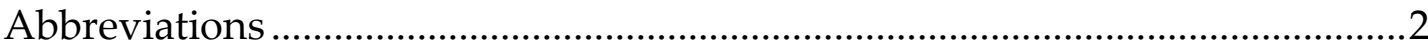

Part A Role of the unconventional Rap1 GEF ELMO-Sponge in the formation of the subapical domain ..........................................................................4

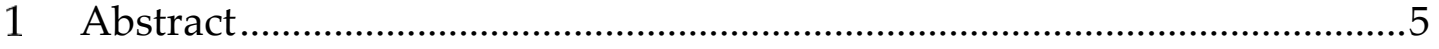

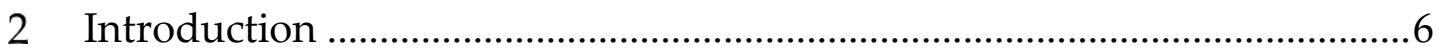

2.1 Cortex and Cortical domains ..........................................................6

2.2 Dynamics of cortical domains in early Drosophila embryos .................8

2.3 Cap and intercap regions in syncytial blastoderm embryos ..............10

2.4 Cortical domains in the metaphase furrow ........................................12

2.5 Cortical organization during cellularization......................................13

2.6 Establishment of the basal domain....................................................... 15

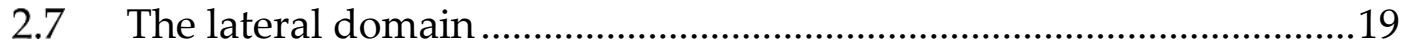

2.8 Emergence of the subapical domain ................................................19

2.9 Transition to epithelial organization during gastrulation ..................22

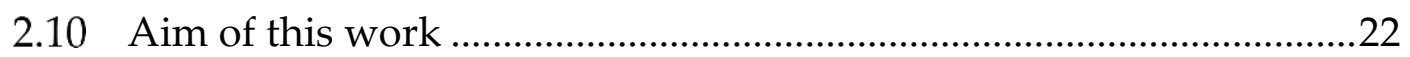

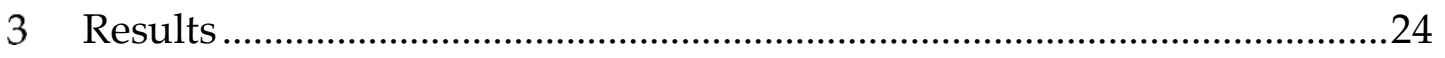

3.1 The organization of cortical domains changes with onset of

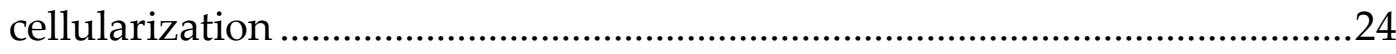

3.2 The unconventional GEF ELMO-Sponge complex controls subapical

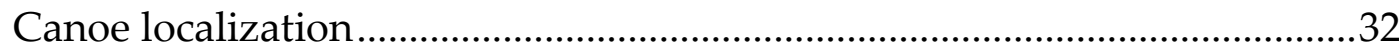

3.3 ELMO-Sponge localization changes from disc-like to ring-like with onset of cellularization

3.4 Bazooka gets enriched at the subapical domain during cellularization

3.5 Bazooka is required for subapical localization of Canoe 47

3.6 The onset of zygotic gene expression is necessary for correct localization of Canoe 49

4 Discussion .53

Part B Role of Dia for the formation of cortical domains and organization of cortical actin 60

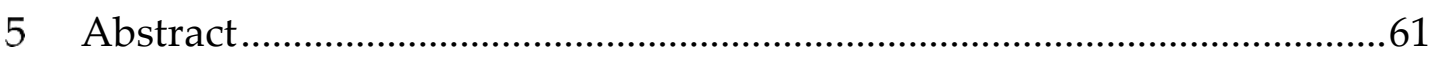

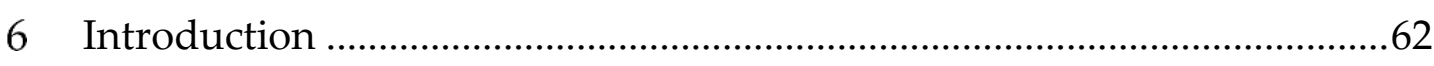

6.1 Actin dynamics are controlled by actin binding proteins ...................62 
6.2 The formin Dia nucleates and elongates filamentous actin and binds to plus ends 63

6.3 Multiple functions of Dia in Drosophila development ......................... 64

6.4 Aim of the thesis...................................................................................... 64

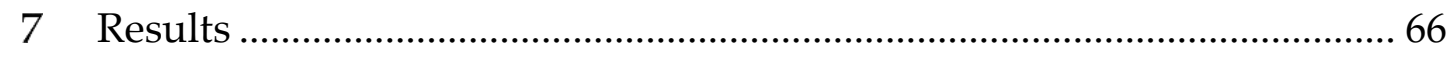

7.1 dia and bazooka show antagonist genetic interaction.............................. 66

7.2 Dia is required for subapical localization of marker proteins ........... 69

7.3 Dia and F-actin are enriched at the basal domain during cellularization ........................................................................................... 78

7.4 Diffusion of Canoe and integral membrane proteins is not affected in

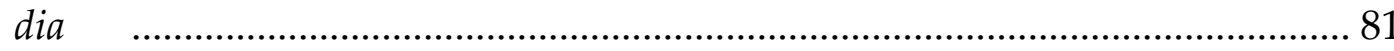

7.5 The effect of endocytosis on the formation of the subapical domain is

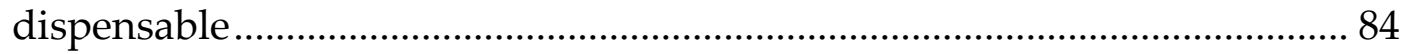

7.6 Cortical F-actin is polarized and this organization depends on Dia 88

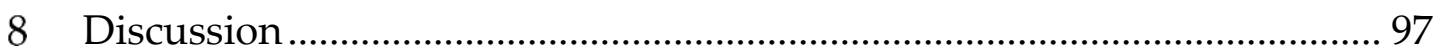

8.1 Introduction ................................................................................... 97

8.2 Dia acts indirectly on subapical cues................................................... 97

8.3 Less Dia does not lead to less cortical F-actin ....................................... 98

8.4 The polarization of cortical F-actin defines the interphase of cap and

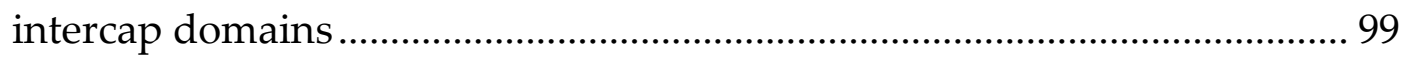

8.5 Midblastula transition as a switch for introduction of a new domain . 101

Part C Role of FMNL during early Drosophila embryogenesis.................. 103

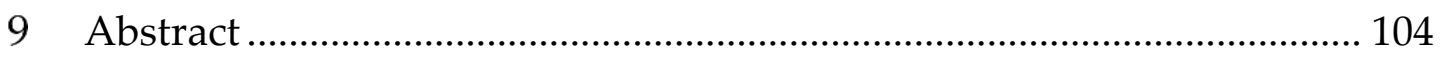

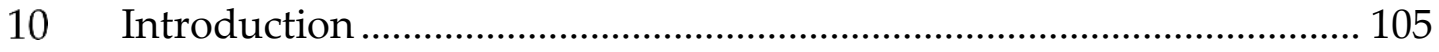

10.1 Proteins of the formin family in Drosophila ........................................ 105

10.2 Formin-like (FMNL) is the sole homolog in Drosophila ................... 105

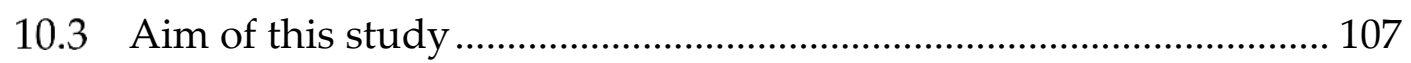

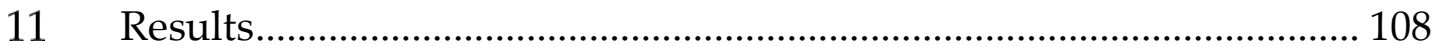

11.1 FMNL was found to be an interactor of Slam by Yeast-Two-Hybrid-

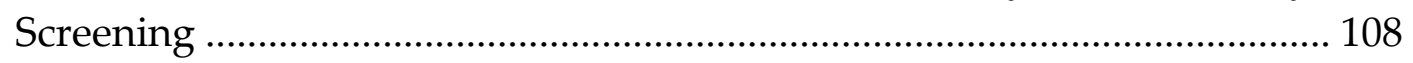

11.2 Embryos from P/Def flies show reduced amount of FMNL-RNA and

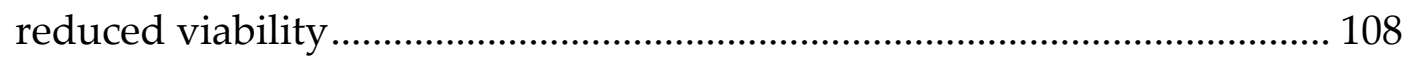

11.3 The purification of an FMNL-antibody did not lead to a specific signal

11.4 FMNL is expressed during embryonic development 
11.5 FMNL is expressed in larval and adult tissues................................119

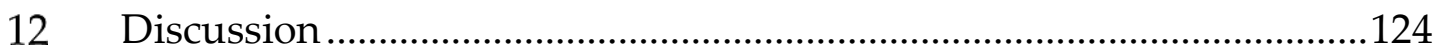

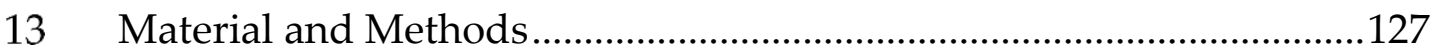

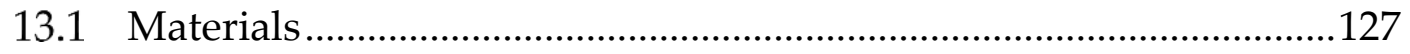

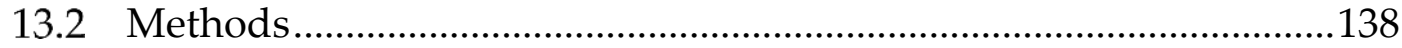

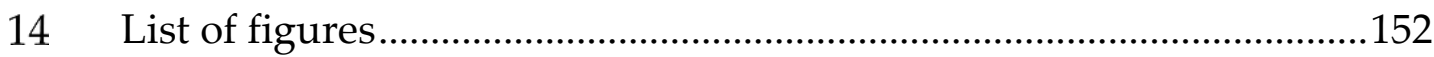

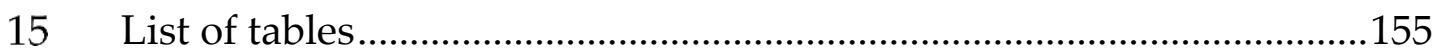

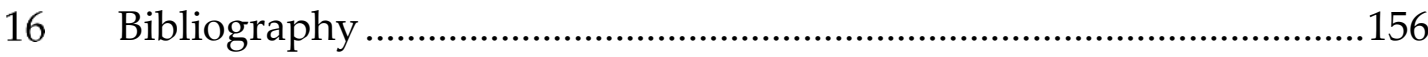

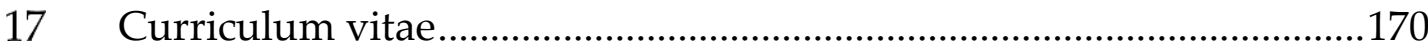

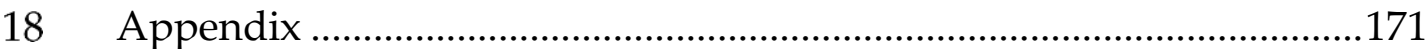




\section{Acknowledgements}

I would like to thank Prof. Dr. Jörg Großhans for giving me the opportunity to carry out my $\mathrm{PhD}$ in his group. I am very thankful for being always available for discussions, answering questions and for always being positive. I also would like to thank my thesis committee Prof. Dr. Reinhard Schuh (MPI-BPC) and Prof. Dr. Robert Grosse (Philipps-University Marburg) for fruitful and helpful discussions.

Furthermore, I would like to thank Prof. Dr. Mark Peifer, Dr. Teresa Bonello and Kia Perez-Vale (University of North Carolina) for discussions and sharing results and materials. I am also grateful to Prof. Dr. André Fiala (University Göttingen), Dr. Thomas Riemensperger and Dr. Carlotta Martinelli for allowing to use the two-photon microscope and for their introduction and help. I am thankful to Prof. Dr. Gaia Tavosanis and Tomke Stürner (DZNE, Bonn) for their stainings of larval brains. I want to thank the DFG for funding and members of the SPP1464 for fruitful discussions and advice.

I would like to thank current and former colleagues of the Department for Developmental Biology for the nice working atmosphere. I am especially grateful to the fly group. I am thankful to Dr. Shuling Yan and Dr. Zhiyi Lv for early work on Dia. A special thank goes to Dr. Zhiyi Lv for the work on ELMO, sharing results, his advice and answering a lot of questions during all 5 years of my PhD. I am thankful to Dr. Zhiyi Lv, Dr. Deqing Kong, Dr. Boyang Liu and Long Li for all the practical help with fly crosses, plate changing and heat shocks. I furthermore would like to thank Dr. Sreemukta Acharya, Stephanie Gröning and Dr. Zhiyi Lv for help with protein purification and Dr. Shuling Yan for help with in situ hybridizations. I really enjoyed working in this group, sharing results, getting advice and open discussions. I am also grateful to Johannes Sattmann for taking care of the microscopes. Furthermore, I want to thank him and our "HiWis" for the preparation of media, fly food and their work in the stock collection.

A special thank goes to my parents and my sister for their support, open arms and believe in me. And I want to thank Torben Erichsen for his support and love. 


\section{Abbreviations}

${ }^{\circ} \mathrm{C}$

$\alpha$

$\mu$

a.u.

aPKC

Arm

Baz

cAMP

cDNA

Cno

CNS

Cpa

DAD

DAPI

Dia

DID

Dlg

DNA

DOCK

Dzy

ELMO

EMS

ER

$\exp$

F-actin

FH

FLIP

FMNL

FRAP

GAP

GDP

GEF

GFP

glc

GTP

h

int.

kDa

L

m

m

Mbc degree Celsius

alpha

micro

arbitrary unit

atypical protein kinase $\mathrm{C}$

Armadillo

Bazooka

cyclic adenosine monophosphate complementary DNA

Canoe

central nervous system

Capping protein alpha

Dia autoregulatory domain

4',6'-Diamidino-2-phenylindole

Diaphanous

Dia inhibitory domain

Discs-large

deoxyribonucleic acid

dedicator of cytokinesis

Dizzy

Engulfment of cell mobility

ethyl methanesulfonate

endoplasmic reticulum

exponential

filamentous actin

Formin homology

Fluorescence loss in photobleaching

Formin-like protein

fluorescence recovery after photobleaching

GTPase-activating proteins

Guanosine diphosphate

Guanine nucleotide exchange factor

green fluorescent protein

germ line clone

Guanosine-5'-triphosphate

hour

intensity

kilo Dalton

liter

meter

milli

Myoblast city 
MBT

min

mRNA

MyoII

n

N

nls

PBS

PCR

PIP

RBD

RE

rel.

RNA

RT-qPCR

S

Scrib

Shi

Slam

Spg

Utr

WT

YFP midblastula transition

minute

messenger RNA

Myosin II

nano

Number of

nuclear localization signal

Phosphate buffered saline

polymerase chain reaction

Phosphatidylinositol phosphate

Rho binding domain

recycling endosome

relative

ribonucleic acid

real time quantitative PCR

second

Scribbled

Shibire

Slow as molasses

Sponge

Utrophin

wild type

yellow fluorescent protein 


\section{Part A}

Role of the unconventional Rap1 GEF ELMOSponge in the formation of the subapical domain 


\section{Abstract}

The formation of the subapical domain during cellularization in Drosophila embryogenesis is mediated by a pathway including the proteins Rap1, Canoe and their downstream effectors Bazooka and Armadillo. The cortical domain at the subapical position gives rise to the zonula adherens with adherens junctions after cellularization. The timing of the subapical domain formation remained unclear. It is possible that the introduction happens gradually with ongoing cellularization or that the domain is introduced at the beginning of cellularization. The localization of Bazooka leads to the first hypothesis as it gets enriched gradually at the subapical domain over the course of cellularization. However, I could show that Bazookas upstream factor Canoe localizes to the subapical domain already during early cellularization. Furthermore, I could show by live imaging with CanoeYFP and the basal marker CherrySlam, that the segregation of subapical and basal domains happens during the first minutes of cellularization.

The upstream factor of Canoe Rap1 localizes to all cortical domains without an enrichment at the subapical domain and the factor activating the GTPase locally remained unclear. I could show that the unconventional Rap1 GEF ELMO-Sponge localizes to the subapical domain and that the formation of this domain is perturbed in ELMO and sponge mutants. This led to the hypothesis, that ELMO-Sponge locally activates Rap1 leading to the formation of the subapical domain. ELMO-Sponge both localize to actin caps before cellularization in a disc-like fashion, labeling the whole cap. This localization changes with onset of cellularization to a more ring-like pattern defining the position for the formation of the subapical domain. I further could show, that the introduction of the subapical domain depends on midblastula transition and with this onset of zygotic gene expression. In embryos in which zygotic gene expression was repressed by injection of the drug $\alpha$-amanitin, cellularization was blocked and subapical Canoe localization was lost. 


\section{Introduction}

The introduction, including Figures 1-4, has been published as an invited review by JCS in Schmidt and Großhans (2018) "Dynamics of cortical domains in early Drosophila development".

\subsection{Cortex and Cortical domains}

Below the plasma membrane in eukaryotic cells, an actin cortex containing a meshwork of actin filaments and associated proteins can be found. In all polarized and epithelial cells, cortical domains exist, which are characterized by specific sets of proteins, and, typically, these are the apical, subapical, lateral and basal domains. These sets of domain-specific proteins contain celltype-specific proteins, as well as proteins that are conserved throughout evolution, among which are the Par proteins, which had originally been identified in Caenorhabditis elegans based on their function in establishing zygotic anterior-posterior polarity (reviewed in Lang and Munro, 2017), the adherens junctions complex of the zonula adherens and markers for the lateral domain, Scribbled (Scrib), Discs large 1 (Dlg) and Lethal giant larvae (Lgl) (reviewed in Campanale et al., 2017).

Among the proteins in the cortex are cortical proteins and actin-associated proteins, such as nucleators, crosslinkers and motors, as well as integral membrane proteins (Figure 1A) (reviewed in Honigmann and Pralle, 2016). The cortex is able to react to external and internal signals and has important functions in cell division, motility, cell shape changes, cell rearrangement and mechanical stability. Cortical domain organization is linked to cell polarity and is important for cell behavior, and, consequent with this, tissue morphogenesis and embryonic development, in a variety of species (reviewed in Munjal and Lecuit, 2014). Cortical domains are set up by the differential localization of proteins that confer identity to cortical domains and are maintained, for example, by lateral diffusion barriers (Figure 1A) (reviewed in Honigmann and Pralle, 2016). Besides their function in epithelial cells, Par proteins also define anterior-posterior polarity in the C. elegans zygote and Drosophila oocyte (reviewed in Nance and Zallen, 2011), as well as separating the inner and outer cells in early mouse embryos, which give rise to the first cell lineages (Korotkevich et al., 2017) (Figure 1). Further functions of cortical domains in non-epithelial cells include axon specification and polarization of neurons, for example, with Par-3 [Bazooka (Baz) in flies] and Par-6 proteins 
being restricted to the apical tip growth cone of axons (reviewed in Insolera et al., 2011), and directed migration of astrocytes, where localization of the Par complex to the leading edge is seen (reviewed in Suzuki and Ohno, 2006) (Figure 1B).
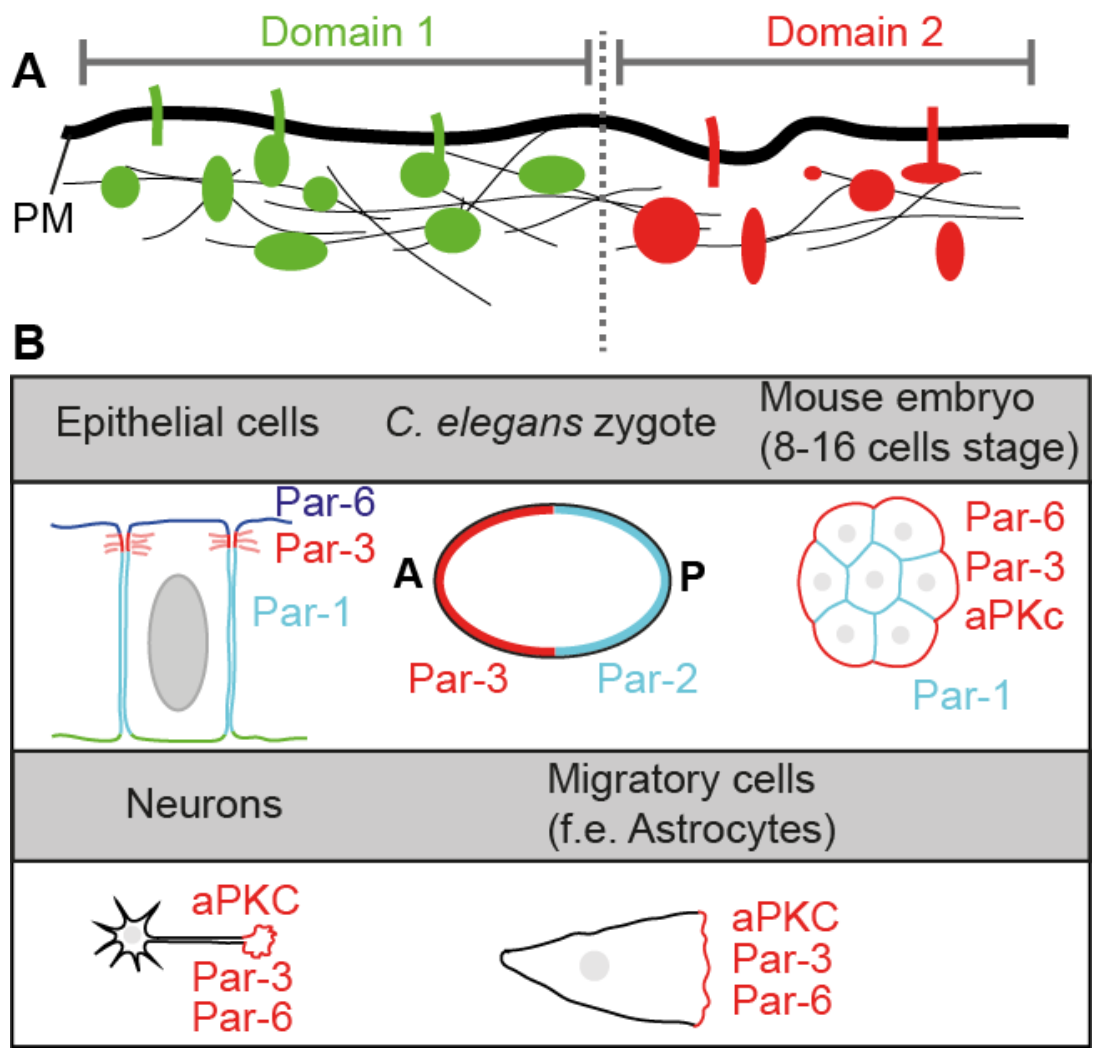

Figure 1 Cortex and cortical domains.

(A) Schematic representation of the cortex. A thin layer of F-actin lies below the plasma membrane, which includes myosin motors, regulators, nucleators and crosslinkers of F-actin. Embedded in the cortex are specific components that are associated with or integrated in the plasma membrane. Different cortical domains are generated by domain-specific sets of cortical components. Lateral diffusion of cortical domain components across the domain boundary is inhibited as shown by the dashed line. (B) Examples of cortical domains. Epithelial cells show a typical distribution of Par proteins with Par-1 localizing to the lateral domain (light blue) and Par-3 to the zonula adherens with adherens junctions (red) (Harris, 2012). In the C. elegans zygote, anterior-posterior polarity is defined by two cortical domains, with Par-3 localizing to the anterior cortical domain (red) and Par-2 defining the posterior half of the zygote (light blue) (Nance and Zallen, 2011). In eight-cell stage mouse embryos, apical-basal polarity becomes defined by the localization of Par-3, Par-6 proteins and aPKC to apical domains (red), whereas Par-1 localizes to the baso-lateral cortex (light blue) (Korotkevich et al., 2017; Vinot et al., 2005). Neurons show polarized cortical domains with aPKC, Par-3 and Par-6 proteins localizing to the apical tip (red) (Insolera et al., 2011). Migratory cells exhibit localization of aPKC, Par-3 and Par-6 to the leading edge (Suzuki and Ohno, 2006).

Several mechanisms for the establishment and maintenance of cortical domains and the molecular factors involved have been delineated. These include mutual exclusion, as the lateral proteins Scribbled, Lgl, Dlg and Par-1, 
exclude apical proteins and adherens junctions from the lateral domain (Bilder et al., 2000; McKinley and Harris, 2012; Tanentzapf and Tepass, 2003; Yamanaka et al., 2006). Directional transport and vesicle trafficking are also assumed to have an important role in the establishment and maintenance of cortical domains. As the generic mechanisms for the establishment and maintenance of typical cortical domains in epithelial cells have been covered in several excellent reviews (Goldstein and Macara, 2007; Krämer, 2000; Laprise and Tepass, 2011; Lecuit, 2004; Mazumdar and Mazumdar, 2002), we will focus here on the dynamic nature of cortical domains and emphasize the relevance of their remodeling in early development of Drosophila.

\subsection{Dynamics of cortical domains in early Drosophila embryos}

Cortical organization and remodeling are tightly linked to embryonic development (Figure 2). From the single uniform cortical domain during the pre-blastoderm stage, the first cortical differentiation takes place during syncytial blastoderm development, where two cortical domains are seen during the interphase and three domains during mitosis (Foe et al., 1993). With mid-blastula transition and the switch to cellularization in interphase 14, a subapical domain is added to give rise to the typical epithelial organization with four cortical domains (reviewed in Harris, 2012).

Following fertilization, the nuclei and their associated centrosomes reside deep within the yolk where they undergo the first nine nuclear cycles. During this stage, the embryonic surface is covered by microprojections of the plasma membrane that are comparable to microvilli (Turner and Mahowald, 1976). The cortex is uniformly organized with an even distribution of F-actin and Myosin II (MyoII) (Karr and Alberts, 1986; Warn et al., 1980; Warn et al., 1984; Young et al., 1991). Cortical Myosin II localization occurs in cycles linked to embryonic mitotic cycles and, along with this, cortical contractions and elongation of the anterior-posterior axis take place (Royou et al., 2002). Staining for endoplasmic reticulum (ER) markers has shown that the cortex is associated with the ER (Frescas et al., 2006) that appears to be organized in a continuous and interconnected membrane system. Fluorescence loss in photobleaching (FLIP) of a cortical ER marker indicates that it is mobile and its diffusion is not delimited by diffusion barriers (Frescas et al., 2006). ER morphology generally depends on microtubules (Terasaki et al., 1986; Waterman-Storer and Salmon, 1998), and, consistent with this, microtubules 
were detected at or close to the cortex (Frescas et al., 2006; Karr and Alberts, 1986). Unpolymerized tubulin and short microtubules that surround small particles, likely yolk granules, can be detected (Frescas et al., 2006; Karr and Alberts, 1986) despite the absence of an obvious microtubule-organizing center (Karr and Alberts, 1986). These microtubules are important for ER localization, as nocodazole treatment leads to a loss of the cortical association of the ER (Frescas et al., 2006). Although the pre-blastoderm cortex has this simple and unstructured organization, it exhibits plasticity and can actively respond to signals like wounding (Abreu-Blanco et al., 2011, 2014).

No. of cortical

domains

1

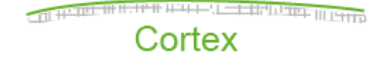

2

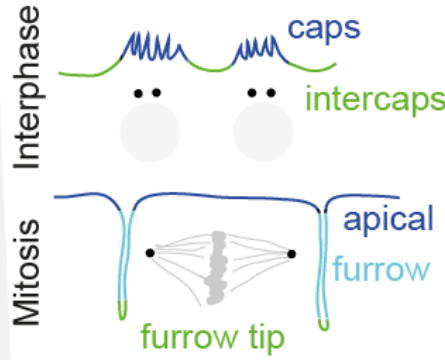

4

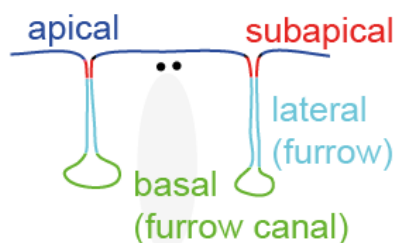

(furrow canal)

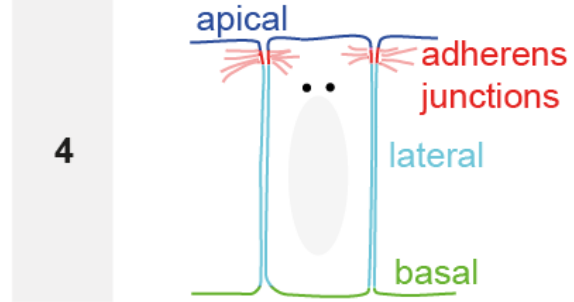

Developmental stage

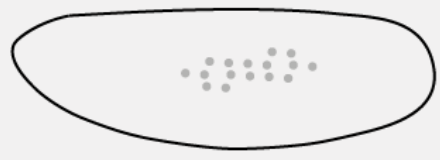

Preblastoderm nuclear cycle 1-9

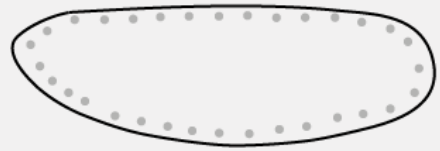

Syncytial blastoderm nuclear cycle 10-13

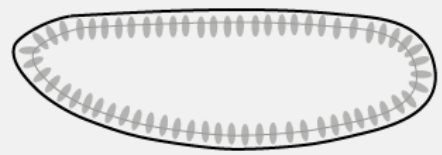

Cellularization cell cycle 14

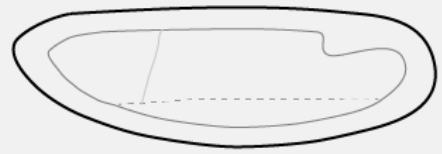

Gastrulation cell cycle 14-

Figure 2 Dynamics of cortical domains in Drosophila embryos.

Schematic representation of cortical domains in early Drosophila embryos in relation to the developmental stages and nuclear cycles. During embryonic development, the number of cortical domains increase from one during pre-blastoderm stage to two (caps and intercaps) in the syncytial blastoderm stage during the interphase and three (apical, lateral and basal) during mitosis. During early cellularization, the new subapical domain emerges between apical and lateral domain. The subapical region matures and contains adherens junctions, which are introduced during gastrulation. 


\subsection{Cap and intercap regions in syncytial blastoderm embryos}

During nuclear cycles 7-9, the nuclei together with their associated centrosomes and cytoplasm migrate from the interior of the yolk towards the cortex (Foe et al., 1993). As soon as the nuclei appear at the cortex, cytoplasmic buds are formed above the nuclei and its associated pair of centrosomes (Figure 2). Although they are most prominent at the anterior pole, the cytoplasmic buds uniformly cover the entire embryonic surface (Foe and Alberts, 1983). This represents the first morphological and molecular differentiation of the embryonic cortex into distinct domains, designated here as caps and intercaps. Within the buds or caps, the plasma membrane forms extended microvilli-like membrane folds (Turner and Mahowald, 1976). Consistent with this, caps are strongly enriched for F-actin (Karr and Alberts, 1986; Kellogg et al., 1988; Warn et al., 1984, 1987), actin-binding proteins such as Arp2/3, suppressor of cAMP receptor (SCAR) (Stevenson et al., 2002; Zallen et al., 2002) and Moesin (Rikhy et al., 2015), as well as proteins functionally related to the actin cytoskeleton, such as spectrins (Thomas and Williams, 1999) and the unconventional guanine nucleotide exchange factor (GEF) complex of ELMO (also named Ced-12 in flies) and Sponge (Spg) (Schmidt et al., 2018). Despite the high F-actin content of caps, Myosin II is not specifically enriched in caps but in intercaps instead (Royou et al., 2002; Warn et al., 1980).

The plasma membrane in the region between the caps (intercaps) appears relatively smooth with only occasional bulbous projections (Turner and Mahowald, 1976) and forms a fold, which becomes more prominent during cycles 12 and 13. In addition to membrane morphology and F-actin content, the separation into two cortical domains is indicated by segregation of marker proteins. GAP43, which attaches to the membrane through a palmitoylated residue (Zacharias et al., 2002), is uniformly distributed over caps and intercaps, whereas Toll (Tl) and Slow as molasses (Slam) segregate to the intercap region (Mavrakis et al., 2009; Schmidt et al., 2018).

The centrosomes are responsible for the segregation of the cortex into caps and intercaps, as there is a strict correlation between emergence of centrosomes with nuclei at the cortex and bud formation (Foe and Alberts, 1983; Karr and Alberts, 1986; Warn et al., 1987). In addition, embryos with 'lonesome' centrosomes (i.e. not associated with a nucleus) are sufficient to induce caps (Peel et al., 2007; Raff and Glover, 1989; Yasuda et al., 1991). 
The link between centrosomes and the cortex is unclear. The increase in F-actin within the caps depends on Arp2/3, which is activated by SCAR (Zallen et al., 2002). SCAR and Arp2/3 activity and, subsequently, actin polymerization in the caps might be controlled through activation of Rac1 by the unconventional GEF complex ELMO-Sponge (Figure 3A). ELMO and Sponge are required for cap formation, as the plasma membrane remains flat without any cytoplasmic buds and an uniformly distributed cortical F-actin in ELMO and sponge mutant embryos (Postner et al., 1992; Schmidt et al., 2018; Winkler et al., 2015). The function of centrosomes at the cortex may involve microtubule-based transport or anchoring, as Kinesin-1 and the Dynein complex are enriched at the caps (Cytrynbaum et al., 2005; Winkler et al., 2015) (Figure 3A). Alternatively, a microtubule-independent mechanism is supported by the observation that the actin caps form even in embryos where the microtubules are depolymerized through treatment with colchicine (Stevenson et al., 2001).

A

Interphase
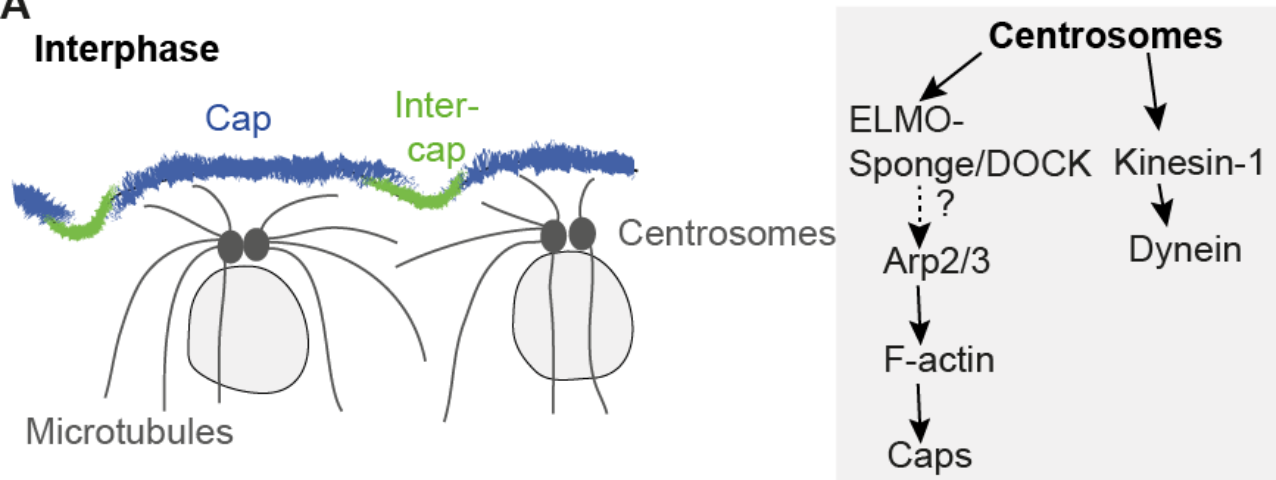

B

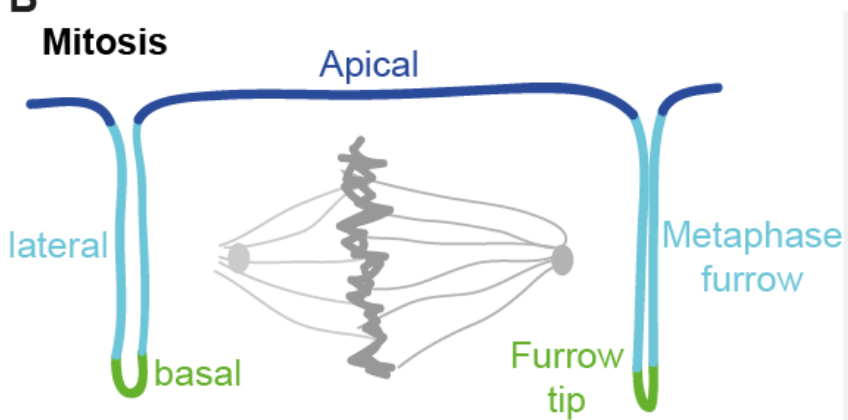

Nuf/ recycling endosome

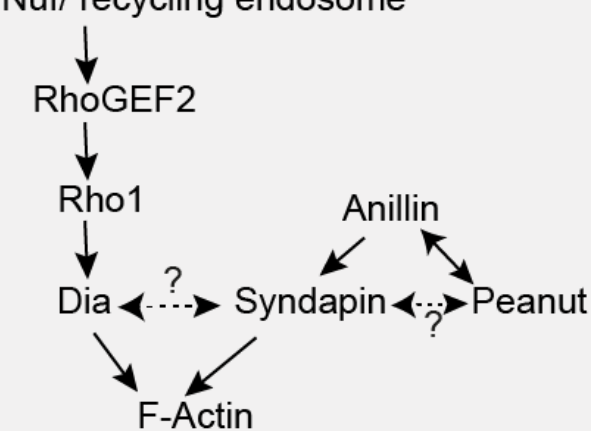

Figure 3 Cortical domains during syncytial blastoderm.

(A) Two cortical domains are present during interphase, named the cap (blue) and intercap (green). The genetic pathways linking centrosomes to domain formation are depicted on the right. (B) Three cortical domains are observed during mitosis, the apical domain (dark blue), lateral domain (metaphase furrow, light blue) and basal domain (furrow tip, green). The genetic pathways responsible for formation of the metaphase furrow are depicted on the right.

The separation into two cortical domains may be linked to the compartmentalization of the plasma membrane, that is, the generation of 
boundaries that limit the movement and spreading of membrane and cortical components between adjacent caps. This has been shown by photobleaching experiments in syncytial blastoderm embryos, in which a cap and its connected intercap region do not exchange cortical and membrane components with the neighboring domains (Mavrakis et al., 2009). The restricted mobility of cortical components depends on the F-actin network, as treatment with latranculin A, which prevents F-actin assembly, alleviated the mobility of cortical markers (Mavrakis et al., 2009).

The differentiation into cortical domains may also be linked to a segregated distribution of phospholipids, as has been observed in generic epithelial cells (reviewed in Gassama-Diagne and Payrastre, 2009; Shewan et al., 2011). However, no such polarized distribution has so far been reported for the syncytial embryo.

\subsection{Cortical domains in the metaphase furrow}

During mitosis 10 to 13 , the individual spindles and their associated chromosomes are separated by transient invaginations of the plasma membrane, termed metaphase or pseudo-cleavage furrows (Foe et al., 1993; Karr and Alberts, 1986) (Figure 2). These transient and dynamic furrows reach a maximum extension of $\sim 10 \mu \mathrm{m}$ during metaphase 13 , and form and retract within a short time frame of $\sim 5$ min (Cao et al., 2008; Karr and Alberts, 1986; Sherlekar and Rikhy, 2016). The metaphase furrows are important for proper chromosome segregation, as mutant embryos that lack the metaphase furrows [e.g. diaphanous (dia) mutants] show a mis-segregation of chromosomes with low frequency (Afshar et al., 2000; reviewed in Sullivan and Theurkauf, 1995).

During mitosis, three cortical domains are present, an apical, lateral and basal domain, as visualized by segregation of respective marker proteins. F-actin and the cortical proteins Amphiphysin (Amph), Anilin, Dia, Syndapin, Myosin II and Patj are strongly enriched at the tip of metaphase furrows (basal domain) (Afshar et al., 2000; Field and Alberts, 1995; Mavrakis et al., 2009; Sherlekar and Rikhy, 2016). Toll and Dlg are found at the lateral furrow and are excluded from the apical side and the basal tip (Cao et al., 2008; Lee et al., 2003; Mavrakis et al., 2009), whereas Canoe, Peanut and Scrambled all localize to lateral and apical domains and are excluded from the basal tip (Harris and Peifer, 2004; Mavrakis et al., 2009; Sawyer et al., 2009; Stevenson et al., 2001). In contrast, the markers GAP43 and the pleckstrin homology domain of 
phospholipase C- $\delta 1$ (PLC 81 ), which binds with high affinity to phosphatidylinositol 4,5-bisphosphate $\left[\mathrm{PI}(4,5) \mathrm{P}_{2}\right]$, are evenly distributed throughout the plasma membrane (Gay and Keith, 1992; Mavrakis et al., 2009; Rikhy et al., 2015). However, the metaphase furrows are highly dynamic, and the reports for protein localization might be incomplete as most of the reports are based on fixed specimens.

Membrane trafficking might be important for the differentiation of cortical domains in the metaphase furrow (Figure 3B). The localization and activity of Dynamin during the syncytial embryo divisions plays an important role in maintaining early embryonic compartmentalization as its inhibition leads to an impaired metaphase furrow and perturbed compartmentalization during interphase (Rikhy et al., 2015). Further evidence for a role of membrane trafficking comes from the observation that the F-BAR protein Syndapin is involved in the maintenance and organization of the metaphase furrow, as syndapin mutants have short metaphase furrows with mislocalized Peanut, Dia and Amphiphysin, leading to a misorganized F-actin network (Sherlekar and Rikhy, 2017).

The lack of metaphase furrows in embryos mutant for dia could be explained by the function of this formin in nucleating and elongating F-actin (Yan et al., 2013). Indeed, several studies show that proper F-actin polymerization is required for the elongation of the metaphase furrow (Cao et al., 2008; Webb et al., 2009), which is mediated by Dia and its activator RhoGEF2 (Großhans et al., 2005; Padash Barmchi et al., 2005). Interestingly, the correct localization of RhoGEF2 to the furrow has been found to be mediated by RE-derived vesicles, whose transport is dependent on the RE-associated proteins Nuclear fallout (Nuf) and Rab11 (Cao et al., 2008), further pointing to the importance of membrane trafficking in establishing the metaphase furrow (Figure 3B).

\subsection{Cortical organization during cellularization}

Cellularization during interphase 14 is a special stage in fly embryonic development. It mediates the transition from syncytial to cellular development and from a maternal to zygotic control of gene expression (reviewed in Blythe and Wieschaus, 2015; Liu and Grosshans, 2017). This stage is generally referred to as the mid-blastula transition (reviewed in Farrell and O'Farrell, 2014; Yuan et al., 2016). 
In contrast to the preceding nuclear cycles, the plasma membrane forms a stable furrow between adjacent nuclei at the onset of interphase 14 (termed the cellularization furrow). Over the following hour, the furrow slowly ingresses to its final length of $\sim 35 \mu \mathrm{m}$, which encloses each of the cortical nuclei into the resulting individual cells, thereby giving rise to a polarized and single-layered columnar epithelium surrounding the yolk (Foe et al., 1993) (Figure 2).

During initial cellularization, two types of furrows are observed, newly emerging furrows between corresponding daughter nuclei of mitosis 13 and 'old' furrows. The old furrows are derived from metaphase furrows that retract to $\sim 3 \mu \mathrm{m}$ in length (He et al., 2016) before they transform into a cellularization furrow and then ingress in synchrony with the 'new' furrows. For correct positioning of the new furrows, a flow of Myosin II towards the new furrow is required during the first minutes of cellularization, which is mediated by the zygotic gene dunk in an unknown manner (He et al., 2016). Following Dunk-dependent flow, Myosin II is recruited by Slam, which then drives further ingression of the cellularization furrow independently of Dunk (He et al., 2016). As no pre-patterning is present at these sites, de novo polarization of the cortex and the emergence of cortical domains occurs at the new furrows, and a segregation of cortical markers can be observed at the onset of cellularization. Importantly, the difference between old and new furrows vanishes as soon as ingression starts (Acharya et al., 2014).

The cellularization furrow is distinct from the metaphase furrow in several aspects. The metaphase furrow is linked to the mitosis, whereas the cellularization furrow forms in interphase (Foe et al., 1993). Beside a clear difference in the kinetics of elongation and retraction of the furrows, a striking difference is the emergence of a subapical domain, which is introduced as a region between apical and lateral domains.

Similar to the cortical differentiation in syncytial blastoderm embryos into caps and intercaps, the centrosomes also trigger the cortical polarization during cellularization (Acharya et al., 2014). Indeed, lonesome centrosomes are sufficient to induce and organize cellularization furrows, as observed by the segregation of the lateral and basal cortical markers, Dlg and Slam. Accordingly, centrosome ablation inhibits marker segregation, and the basal marker Slam remains distributed along the entire membrane (Acharya et al., 2014), suggesting that centrosomes provide the initial information for cortical 
differentiation and the restriction of cortical markers to their respective domain (Figure 4).
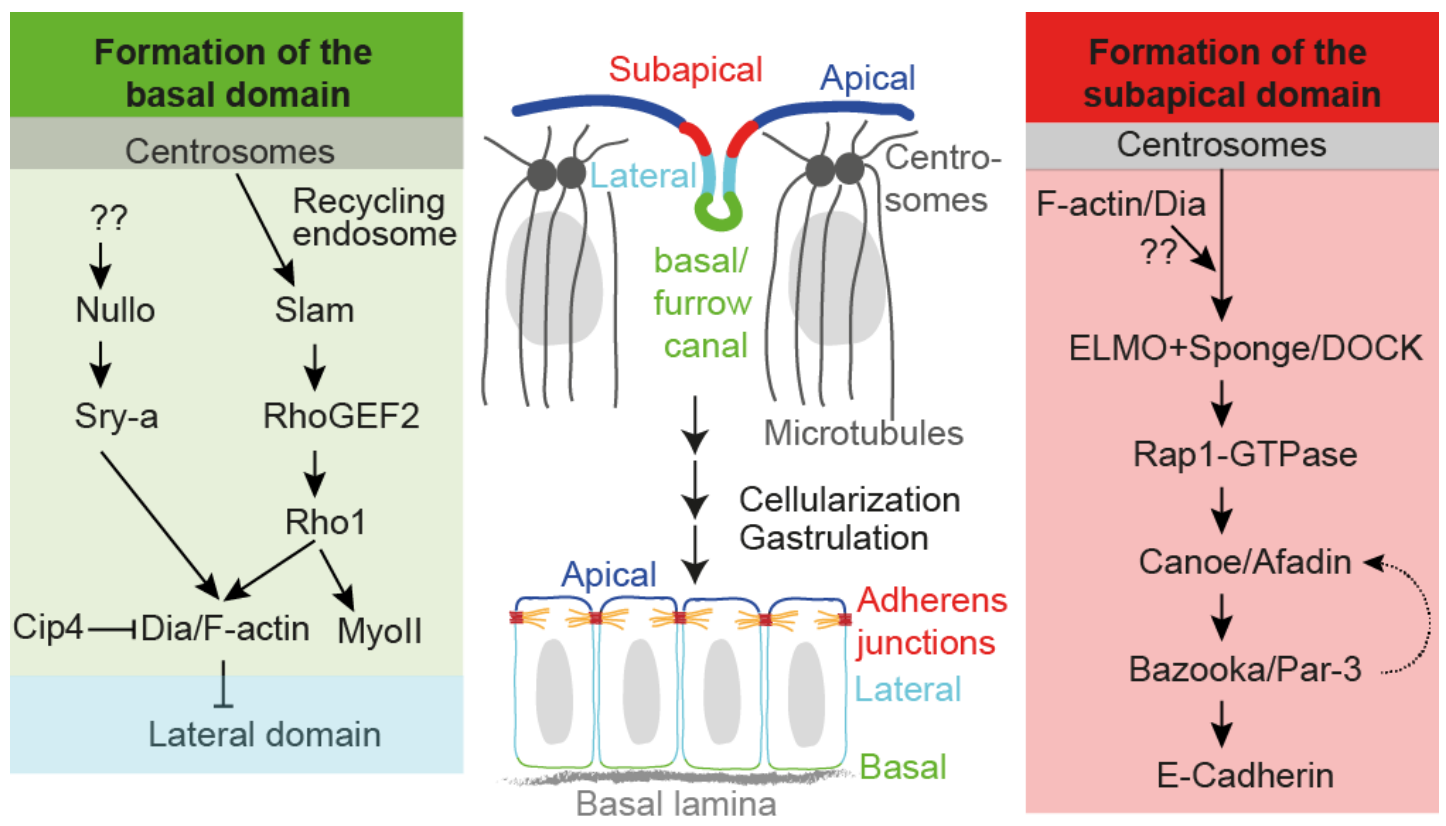

Figure 4 Cortical domains during cellular blastoderm/cellularization.

Schematic illustration of a furrow during early cellularization with apical (dark blue), subapical (red), lateral (light blue) and basal (green, furrow canal) domains depicted. Centrosomes are apical to the nuclei and constitute the anchor for the microtubule basket as depicted. Cellularization results in an epithelium with apical, lateral and basal domains, which is later connected to the basal lamina (gray) and contains adherens junctions (red). The genetic pathways for formation of the basal domain and separation from the lateral domain, and those involved in the formation of the subapical domain are shown on the left and right, respectively.

In the following sections, we will discuss the signaling pathways that contribute to the establishment of the cortical domains that emerge during cellularization in more detail. However, we will not discuss the apical domain as the function of this domain during cellularization has not been studied in detail and no specific marker proteins have been reported yet.

\subsection{Establishment of the basal domain}

The basal domain forms a specific morphological structure. The furrow canal leads the ingressing furrow and will broaden during the second half of cellularization before it finally encloses the adjacent cells (Foe et al., 1993) (Figure 4). In this way, the basal domain of the cellularization furrow is different from the basal tip of the metaphase furrow and also distinct from the generic basal domain of epithelial cells that arises later. 
The basal domain is specified by two redundant signaling pathways (Figure 4). The first pathway is triggered by a complex between Slam and its mRNA, which localizes to the basal domain throughout cellularization (Acharya et al., 2014; Wenzl et al., 2010; Yan et al., 2017). The restriction of Slam to the prospective basal domain depends on recycling endosomes (RE) and the arfophilin Nuf, which is necessary for cycling of Rab11 (Riggs et al., 2003). The requirement for Nuf and/or REs for the exclusion from Slam from the apical and lateral domains is demonstrated by what is seen in nuf mutants, which have impaired and disorganized REs, and in which Slam is uniformly distributed over the plasma membrane (Acharya et al., 2014). In hypomorphic nuf situations, when a furrow forms, Slam is detected at the lateral and basal domain instead of being restricted to the basal domain, indicating that domain segregation is impaired (Acharya et al., 2014). Slam activates Rho signaling by recruiting RhoGEF2 to the prospective basal domain through a physical interaction that involves the PDZ domain of RhoGEF2 and an unconventional PDZ-binding motif within the C-terminal part of Slam (Wenzl et al., 2010) (Figure 4). Downstream of Rho1, actin polymerization is mediated by Dia and actomyosin contractility induced by Myosin II (Afshar et al., 2000; Großhans et al., 2005; Padash Barmchi et al., 2005; Wenzl et al., 2010), resulting in furrow invagination. Furthermore, other polarity markers such as Patj become basally restricted in a manner that depends on their direct or indirect interaction with Slam (Wenzl et al., 2010).

In parallel, a second signaling pathway is established by Nullo, which accumulates in the basal domain, depending on N-terminal myristoylation and an N-terminal cluster of positively charged amino acids (Hunter and Wieschaus, 2000; Postner and Wieschaus, 1994) (Figure 4). Depending on Nullo, Serendipity- $\alpha$ (Sry- $\alpha$ ) becomes restricted to the prospective basal domain (Postner and Wieschaus, 1994; Schweisguth et al., 1990). Nullo and Sry- $\alpha$ control F-actin, possibly also through the formin Dia, as RhoGEF2 nullo and slam nullo double mutants exhibit a stronger phenotype than single mutants, with a uniform distribution of Dia and loss of the basal domain (Acharya et al., 2014; Großhans et al., 2005). Importantly, both nullo and slam are zygotic genes that are expressed early in cellularization (Lecuit et al., 2002; Postner and Wieschaus, 1994), which distinguishes this stage from the preceding nuclear cycles. Therefore, their expression may confer the timing information that controls the new cortical organization in cellularization. 
Both, the Slam and Nullo pathways contribute to specification of the basal domain, as loss of a single pathway, for instance in the single mutants of nullo or RhoGEF2, leads to a dispersed pattern of F-actin with regions that have a proper cortical organization and regions without any specified cortical domains (Acharya et al., 2014; Wenzl et al., 2010). This, in turn, leads to a disrupted furrow array and, ultimately, to the formation of multinuclear cells (Hunter and Wieschaus, 2000; Wenzl et al., 2010). Therefore, the specification of the basal domain appears to be essential for furrow formation and ingression of the plasma membrane.

The basal domain also has a peculiar morphological structure. During the onset of cellularization, dynamic transient tubular extensions in the micrometer range arise from the basal membrane spanning into the cytoplasm as observed by staining with the N-BAR protein Amphiphysin or other markers of the basal domain such as Slam (Sokac and Wieschaus, 2008a; Yan et al., 2013). With progression of cellularization, these tubular extensions disappear. In embryos, in which F-actin is reduced, such as owing to cytochalasin treatment or in dia or nullo mutants, the tubular extensions persist throughout cellularization (Bogdan et al., 2013; Sokac and Wieschaus, 2008a; Yan et al., 2013). Three not mutually exclusive models have been proposed with regard to the dynamics of the tubular extensions. First, that tubules act as membrane reservoirs that regulate furrow ingression rates, given that absence of tubules in amphiphysin mutants leads to increased ingression rates (Su et al., 2013). Second, according to the so-called endocytosis model, that tubular extensions give rise to endocytic vesicles and so promote the turnover of the basal domain, thereby restraining the endocytosis of proteins, such as Peanut, Patj and Myosin II (Sokac and Wieschaus, 2008a). Third, the cortex model suggests that the tubular extensions reflect a weak cortex that allows the infolding of the plasma membrane (Yan et al., 2013). With the formation of the basal domain and progression of cellularization, cortical F-actin builds up and suppresses tubulation in the region of the basal domain (Simpson and Wieschaus, 1990; Sokac and Wieschaus, 2008b). In support of the cortex model, the ultrastructure of the furrow canal in dia mutants shows extensive blebbing and folding, in contrast to the wild-type membrane, which is straight and flat (Großhans et al., 2005). These reports indicate that F-actin produced and organized by Dia is needed to suppress tubulation. Further studies are needed to distinguish between the models, which are not mutually exclusive, in order 
to delineate the function of the tubular extensions and to reveal mechanistic insights.

The ability to segregate the basal markers from the lateral factors appears to be linked to the micrometer-sized tubular extensions within the basal domain. In all situations, in which these tubular extensions persist throughout cellularization, lateral markers were found to invade the basal domain, while the basal domain remained intact, as judged by the restriction of basal markers (Sokac and Wieschaus, 2008a; Yan et al., 2013). Physical barriers within the membrane such as cell-cell junctions are unlikely to contribute to the exclusion of lateral markers, as embryos with impaired E-Cadherin-based junctions are still able to exclude lateral markers (Sokac and Wieschaus, 2008b).

Alternatively, the interaction of the actin cortex with the plasma membrane might mediate the segregation of the basal and lateral domains. Indeed, the FBAR protein Cip4, which links the plasma membrane to the actin cytoskeleton (Fricke et al., 2009), antagonizes basal-lateral domain segregation, as overexpression of Cip4 resulted in the spreading of lateral markers into the basal domain, similar to what is seen for dia mutants (Yan et al., 2013) (Figure 4). Mechanistically, Cip4 has been shown to directly bind to Dia and to inhibit Dia-mediated F-actin nucleation and elongation in vitro (Yan et al., 2013; reviewed in Bogdan et al., 2013). However, it remains unclear how the inhibition of Dia by Cip4 would lead to an exclusion of lateral markers. Dia may promote a stable actin cortex with corresponding lower turnover of the plasma membrane, whereas Cip4 may counteract this. Owing to the large amounts of Dia and F-actin at the basal domain, this model would predict a softer cortex and higher rates of membrane turnover in the lateral domain than in the basal domain, as well as a uniformly soft cortex with high membrane turnover in dia or nullo mutants. However, further research is needed to test whether this hypothesis is indeed true.

Taken together, these findings suggest that two pathways triggered by the zygotically expressed proteins Slam and Nullo specify the basal domain and also help to establish a stable actomyosin network that is needed to stabilize the basal furrow and to execute the contractions that eventually close the adjacent cells. 


\subsection{The lateral domain}

The lateral domain, which is located above the basal domain already contains lateral marker proteins (e.g. Scribbled), in metaphase furrows during syncytial nuclear cycles as discussed above. With the formation of the cellularization furrow at the position between the daughter nuclei of the last nuclear division, a new lateral domain will arise (Schmidt et al., 2018). Similar to their localization in the metaphase furrow, Dlg, Scribbled and Lgl mark the region apical to the basal domain (Bilder et al., 2000). However, they do not function in furrow ingression or the formation of the subapical domain during early cellularization, as the localization of the subapical marker Canoe is not affected in scribbled mutants (Schmidt et al., 2018). In fact, the function for the DlgScribbled-Lgl complex in cortical organization emerges later (Bilder and Perrimon, 2000; Bilder et al., 2000). Par-1 is uniformly distributed at the cortex during early cellularization, but by mid-to-late cellularization, Par-1 decreases apically and basally and thus becomes restricted to the lateral domain (McKinley and Harris, 2012). The role of Par-1 for cortical domains and cellularization is complex. Par-1-depleted embryos lack some of the cellularization furrows, indicating an early function in furrow ingression. Par1 also functions in clearing Bazooka from the lateral domain as Bazooka spreads into the lateral furrow in Par-1-depleted embryos (McKinley and Harris, 2012). As Par-1 is excluded from the subapical domain only late in cellularization, it is likely that clearing of Bazooka (Baz) from the lateral domain by Par- 1 is a gradual process that functions in addition to Canoe (Cno)-dependent subapical recruitment of Bazooka.

Taken together, cortical domains appear to be formed largely independently of each other in early cellularization. During the course of cellularization, however, they mutually interact, which then leads to lateral exclusion of Bazooka from the lateral domain, for example.

\subsection{Emergence of the subapical domain}

The lateral, basal and apical domains all have predecessors in the cap and intercap regions, or the metaphase furrow during the syncytial blastoderm. However, the subapical domain is special in that it emerges between the apical and lateral domains as a new feature during cellularization.

The subapical domain is specified by a signaling pathway that involves the small GTPase Rap1 and the actin-binding protein Canoe (Afadin in mammals) 
(Figure 4). It controls the subapical localization of the conserved markers Bazooka (Par-3 in mammals), Par-6 and atypical protein kinase C (aPKC), as well as accumulation of the complex between E-Cadherin, Armadillo (Arm) and $\alpha$-Catenin during the course of cellularization through an unknown mechanism (Choi et al., 2013; reviewed in Harris, 2012) (Figure 4). Whereas Canoe is restricted to the subapical domain from the onset of cellularization (Schmidt et al., 2018), Bazooka and E-Cadherin only gradually accumulate there (Harris and Peifer, 2004). A prominent subapical localization of Bazooka and the E-Cadherin complex is only apparent by the end of cellularization. The subapical accumulation of Bazooka depends on cytoskeletal cues, such as binding to an actin scaffold and Dynein-mediated transport in basal to apical direction (Harris and Peifer, 2005).

Although it is known that Canoe is needed for proper subapical localization of Bazooka (Choi et al., 2013), it is still unclear how Bazooka is recruited by Canoe (Figure 4). Canoe might recruit Bazooka to the subapical domain by direct binding, as supported by a protein recruitment assay performed in S2 cells (Choi et al., 2013). Alternatively, Canoe might act indirectly and/or transiently with Bazooka, as they do not show obligatory colocalization during cellularization (Choi et al., 2013). For instance, Canoe could control the microtubule-dependent apical transport of Bazooka through an unknown mechanism (McKinley and Harris, 2012). Another model has been proposed based on the recruitment of Bazooka by membrane lipids, as Bazooka contains a PH domain in its C-terminus (Krahn et al., 2010a,b). However, as no specific subapical enrichment of phosphatidylinositols (PIPs) has been detected (Reversi et al., 2014), it is unlikely that these phospholipids are involved in the subapical restriction of Bazooka during cellularization.

The source of the information that initially positions the subapical domain between the apical and lateral domains is unknown. The GTPase Rap1 is known to act upstream of subapical protein Canoe as restricted Canoe localization during cellularization is lost in Rap1 mutant embryos (Sawyer et al., 2009). Rap1 requires an initial signal for positioning Canoe to the newly emerging subapical domain, as it is uniformly distributed over the entire membrane (Sawyer et al., 2009). Rap1 activation is most likely spatially restricted, which subsequently leads to the subapical restriction of Canoe (Figure 4). Indeed, the expression of a constitutively active form of Rap1 leads 
to mislocalization of Canoe, as well as of Bazooka and Arm, to the lateral domain (Bonello et al., 2018).

The dynamics and function of the upstream regulators of Rap1 may provide clues to the origin of the positional information, and multiple GEFs and GTPase-activating proteins have been described for Rap1. A promising candidate is the GEF Dizzy (Dzy), which has been shown to be required for Rap1 activity in the assembly of apical adherens junction in the mesoderm anlage (Spahn et al., 2012). During late cellularization, Dizzy is involved in the localization of Canoe to tricellular junctions (Bonello et al., 2018), but not in its subapical restriction (Schmidt et al., 2018). This latter function appears to be fulfilled by the ELMO-Sponge complex, which is an unconventional GEF, as subapical restriction of Canoe is perturbed in ELMO and sponge mutant embryos (Schmidt et al., 2018). It is assumed that ELMO (Ced-12) provides the $\mathrm{PH}$ domain for membrane association and Sponge (the homolog of mammalian Dock180) entails the enzymatic activity and confers specificity for Rap1 and also Rac (Biersmith et al., 2011; Komander et al., 2008; Yajnik et al., 2003). The ELMO-Sponge complex is enriched at the prospective subapical domain during the onset of cellularization (Schmidt et al., 2018), so the complex could provide local activation of Rap1 and, through this, spatial information for the introduction of the subapical domain (Figure 4).

Although a signaling pathway involving ELMO-Sponge has been defined to act through Rap1 to restrict Canoe, and consequently Bazooka and ECadherin, to the subapical domain, the mechanism that changes the distribution of the ELMO-Sponge complex during onset of cellularization is much less clear. Strikingly, the localization of ELMO-Sponge changes from a disc-like pattern at the caps in the syncytial blastoderm to a ring-like pattern during onset of cellularization. The molecular basis for this is unknown but is likely to be linked to the structure and dynamics of actin caps and to the midblastula transition and possibly newly transcribed zygotic factors (Schmidt et al., 2018).

An important open question is the role of the cytoskeleton. The subapical restriction of Bazooka and Canoe requires F-actin assembly, as drug-induced F-actin depolymerization results in the dispersion of Canoe and Bazooka (Choi et al., 2013; Harris and Peifer, 2005). However, a direct function of F-actin in the positioning of subapical cues is unlikely, as F-actin is not visibly enriched 
at the subapical domain during early cellularization, but instead accumulates only later in development at adherens junctions (Choi et al., 2013).

\subsection{Transition to epithelial organization during gastrulation}

The make-up of the cortex in generic epithelia includes the apical, lateral and basal domains. The region between the apical and lateral domain is further differentiated into the extreme apical region or marginal zone as marked by Crumbs and the region containing the adherens junctions (Harris and Peifer, 2004; reviewed in Tepass, 2012). However, this organization is partially independent of the cortical organization that is set up during cellularization. Mutants that exhibit an impaired subapical domain during cellularization such as canoe or Dynein heavy chain $64 \mathrm{C}$, recover during gastrulation with a clearly subapically restricted localization of Bazooka and E-Cadherin (Choi et al., 2013; Harris and Peifer, 2005). It appears that upon transition from cellularization to gastrulation, the conserved components for epithelial and cortical organization, such as the Par proteins, take over control from the cellularization-specific mechanism based on Rap1 and Canoe.

Such a transition is also obvious in the dynamics of the subapical marker proteins. Bazooka and E-cadherin localize to a more apical position where they form the zonula adherens (reviewed in Harris, 2012), and the localization of Bazooka becomes independent of Canoe (Choi et al., 2013). During gastrulation, Bazooka localization is mainly governed by the mutual exclusion of factors between the different cortical domains, in that, Bazooka is excluded from the lateral domain by the presence of Par-1 (McKinley and Harris, 2012), and from the apical domain by Par-6 and Crumbs (Bilder and Perrimon, 2000; Hutterer et al., 2004; Krahn et al., 2010a, b; Morais-de-Sá et al., 2010). Similarly, the lateral proteins Dlg, Scribbled and Lgl are important for the lateral exclusion of subapical and apical proteins (Bilder and Perrimon, 2000; Bilder et al., 2003; Hutterer et al., 2004; Tanentzapf and Tepass, 2003). We will not cover this aspect here in more detail, as several excellent reviews have recently been published addressing epithelial organization (e.g. (Coopman and Djiane, 2016; Harris, 2012; Laprise and Tepass, 2011; Nance, 2014).

\subsection{Aim of this work}

The formation, dynamics and maintenance of cortical domains are crucial for morphogenesis and functionality of differentiated cells and tissues. Cortical domains are linked to cell polarity and remodeling of adherens junctions 
during mesenchymal-epithelial transition and vice versa. The understanding how cortical domains form can help to understand these processes better. However, the formation of cortical domains in an epithelium can only be analyzed in a forming epithelium, which makes the process of cellularization during Drosophila development to a suitable model. Cellularization is especially suited to investigate the formation of epithelial domains because it allows to track the segregation of marker proteins with stereotypic timing.

Although, the pathway for the formation of adherens junctions is well understood, the formation of the preceding subapical domain was not well investigated yet. In the first part of my thesis I defined the timing of subapical Canoe accumulation and segregation with the lateral marker Scribble and the basal marker Slam. Furthermore, I was able to show, that the unconventional GEF complex formed by ELMO-Sponge is acting upstream of Rap1 and responsible for its local activation during onset of cellularization leading to the formation of the subapical domain. 


\section{Results}

Large parts of this results section have been published by Development in Schmidt et al. (2018) “ELMO and Sponge specify subapical restriction of Canoe and formation of the subapical domain in early Drosophila embryos" including Figures 5-13 and 15-21, 25 and 30.

\subsection{The organization of cortical domains changes with onset of cellularization}

After the fertilization of the Drosophila egg, 9 nuclear divisions take place in the interior of the preblastoderm embryo (reviewed in Foe et al., 1993). During preblastoderm stage, the actin cortex, underlying the plasma membrane, was unstructured and only one cortical domain could be found (Figure 5B) (reviewed in Schmidt and Grosshans, 2018). With nuclear cycle 10 in syncytial blastoderm, when the nuclei reach the periphery of the embryo, cortical domains, which are dependent on the cell cycle, could be detected (Figure 5). During interphase every nucleus was covered by an actin cap formed by membrane infoldings called microvilli. The caps showed enriched F-actin staining (Figure 5A, C) whereas intercap regions were stained by markers such as Slam (Figure 5A, C). During metaphase around $10 \mu \mathrm{m}$ long furrows reach into the interior of the syncytial blastoderm embryo to separate the neighboring spindles from each other. The so-called metaphase furrows were stained by Canoe that localized to apical and lateral regions whereas the lateral membrane was stained by Dlg. Slam stained the basal domain of the furrows (Figure 5A, D). With midblastula transition, the onset of zygotic gene expression and start of cellularization a fourth cortical domain is introduced between apical and lateral domain in interphase 14. During cellularization Canoe stained the newly introduced subapical domain, whereas it was restricted from apical and lateral domains (Figure 5A, E). Lateral and basal domains were stained by Dlg and Slam. This domain organization with four domains was stable throughout cellularization (Figure 5A, F). After cellularization, the subapical localized Canoe leads the way for setup of adherens junctions to build an epithelium with apical, lateral and basal cortical domains and the zonula adherens between lateral and apical domain (reviewed in Harris, 2012). 


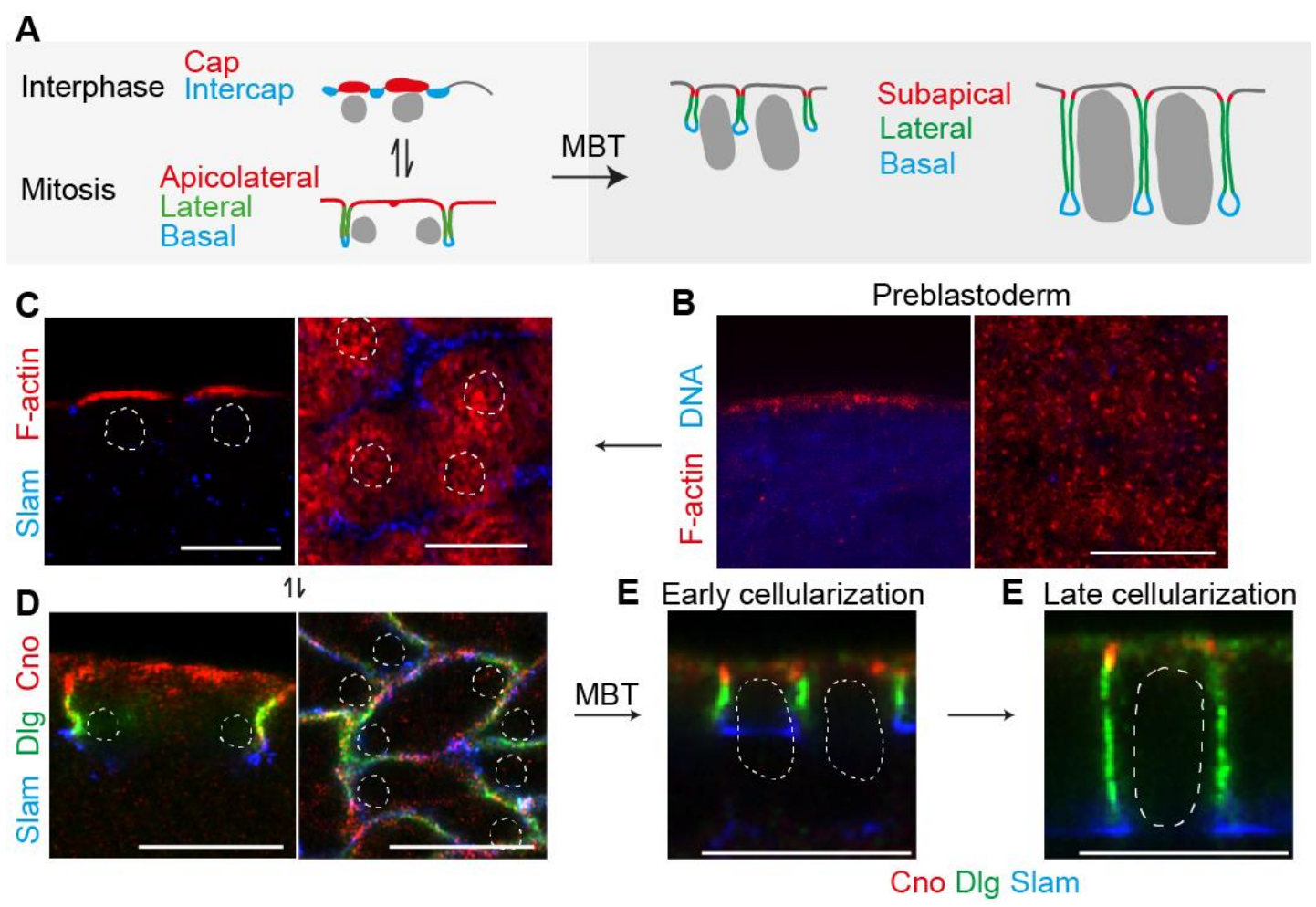

Figure 5 Dynamics of cortical domains in the Drosophila blastoderm embryo.

(A) Scheme (sagittal view) illustrating cortical domains before and after midblastula transition (MBT). Cap, apicolateral and subapical domains are marked in red, intercap and basal domain, in blue and lateral domain, in green. (B-F) Images of embryos stained for domain marker before and after MBT. (B) Preblastoderm embryo stained for F-actin (red) and DNA (blue) in sagittal and planar view (C) Interphase 13, stained for caps (F-actin, red) and intercap regions (Slam, blue) in sagittal and planar view. (D) Mitosis 12, stained for the apical-lateral (Canoe, red), lateral (Dlg, green) and basal domains (Slam, blue). (E, F) Early (E) and late (F) cellularization (interphase 14), sagittal view, stained for subapical (Canoe, red), lateral (Dlg, green) and basal domains (Slam, blue). Dashed lines represent nuclei. Scale bars $10 \mu \mathrm{m}$.

\section{Canoe marks the subapical domain with onset of cellularization}

With the staining of Canoe and Slam in fixed embryos, I could show, that both proteins were functional markers for the subapical and basal domains. To further analyze the formation of the subapical domain and the separation from the basal domain in living embryos during cellularization, I made use of YFPand mCherry-tagged versions of both proteins. I used embryos, expressing CanoeYFP and CherrySlam and imaged them during the transition from metaphase 13 and interphase 14, during which cellularization takes place. To show the introduction of the new subapical domain I imaged z-stacks from the top view with a step size of $0.5 \mu \mathrm{m}$ and in intervals of one minute (Figure 6). Timepoint zero was defined by the emergence of the new furrow between a pair of daughter nuclei. Figure 6 shows merged images of an embryo expressing CanoeYFP and CherrySlam. During the end of metaphase 13 (timepoint-1) CanoeYFP could still be detected along the whole lateral 
domain of the metaphase furrow, which was still retracting at beginning of interphase 14 to a length of around $5 \mu \mathrm{m}$. With beginning of interphase, the new furrow was immediately marked with CanoeYFP (0 min) whereas the basal marker CherrySlam was not yet visible at the new furrow (Figure 6).

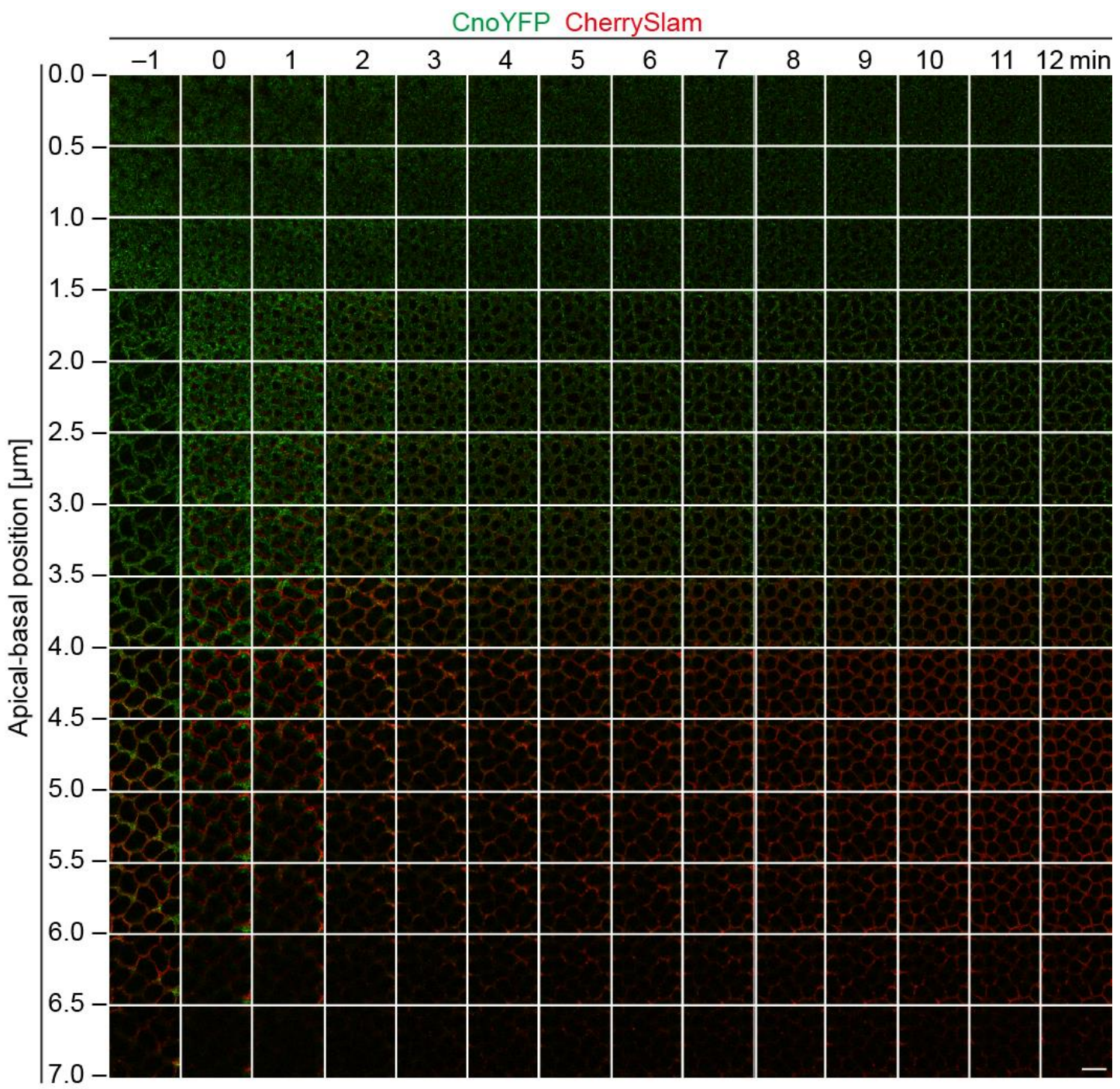

Figure 6 CanoeYFP and CherrySlam dynamics during mitosis 13 and interphase 14 .

Images from time lapse recording of an embryo expressing CanoeYFP (green) and CherrySlam (red). Time from left to right, apical basal position from up to down. Scale bar $10 \mu \mathrm{m}$.

To get a better insight into the separation of subapical and basal domain, I took fewer timepoints and significant z-positions from Figure 6 and separated the channels from each other (Figure 7B). A scheme of the sagittal view during metaphase 13 and the transition to cellularization and the localization of CanoeYFP and CherrySlam is shown in Figure 7A. Figure 7B shows at 0 min, that CanoeYFP marked the new furrow at an apical-basal position of 1.5$3.0 \mu \mathrm{m}$ (upper panel), whereas CherrySlam still marked the basal part of the retracting metaphase furrow at an apical-basal position of 4.0-4.5 $\mu \mathrm{m}$ but the 
new furrow was not marked yet (middle panel). With CanoeYFP signal getting sharper in the subapical region at around $7 \mathrm{~min}$ and an apical-basal position of 1.5-3.0 $\mu \mathrm{m}$, the new furrow further invaginated and CherrySlam got visible at the furrow canal of the new furrow at an apical-basal position of around 4.0$4.5 \mu \mathrm{m}$ (yellow arrowhead).

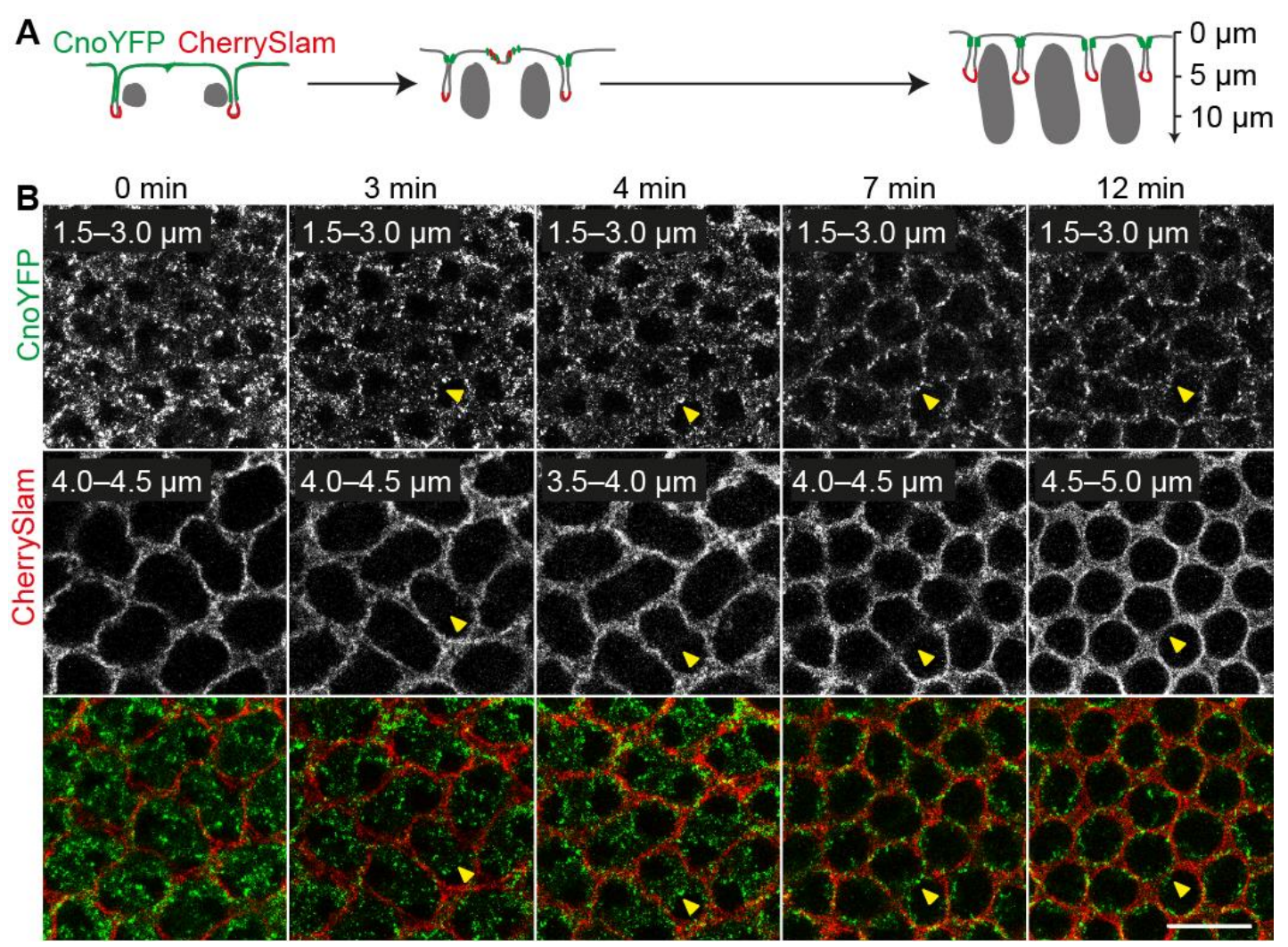

Figure 7 Localization of Canoe and Slam at onset of cellularization.

(A) Scheme for furrow formation and invagination in early cellularization. CanoeYFP (green) and CherrySlam (red) mark subapical and basal domains respectively. Axial (apical-basal) axis with appropriate scale is indicated on right side. (B) Images from time lapse recordings including axial stacks of embryo expressing CanoeYFP (grey/green) and CherrySlam (grey/red) during mitosis 13 and early interphase 14. Axial position is indicated on upper left. Yellow arrowheads point to position of "new" furrows. Scale bar $10 \mu \mathrm{m}$.

To quantify the separation of CanoeYFP and CherrySlam, I measured the fluorescence intensity along the apical-basal axis of three new furrows at $4 \mathrm{~min}$, when the new furrow was not yet marked by CherrySlam and at $8 \mathrm{~min}$, when CherrySlam was clearly visible at the basal furrow canal (Figure 8A, arrowheads depict measured furrows). As comparing the total numbers of fluorescence intensity was not possible, I normalized the numbers for each protein to their peak to get relative values and plotted these values as a graph against the position the apical-basal axis (Figure 8B). A weak CherrySlam signal that did not show a clear peak was already visible at $4 \mathrm{~min}$ (red dashed 
line) whereas CanoeYFP already showed a clear subapical peak at $4 \mathrm{~min}$ (green dashed line), which also stayed subapical (green line) although the new furrow further invaginated as a clear basal peak of CherrySlam (red line) could be detected at $8 \mathrm{~min}$. Both signals were already separated after $8 \mathrm{~min}$ even though the new furrow was, with a length of around $5.5 \mu \mathrm{m}$, still very short. With these results I could show that domain separation takes place during the first minutes of furrow invagination.

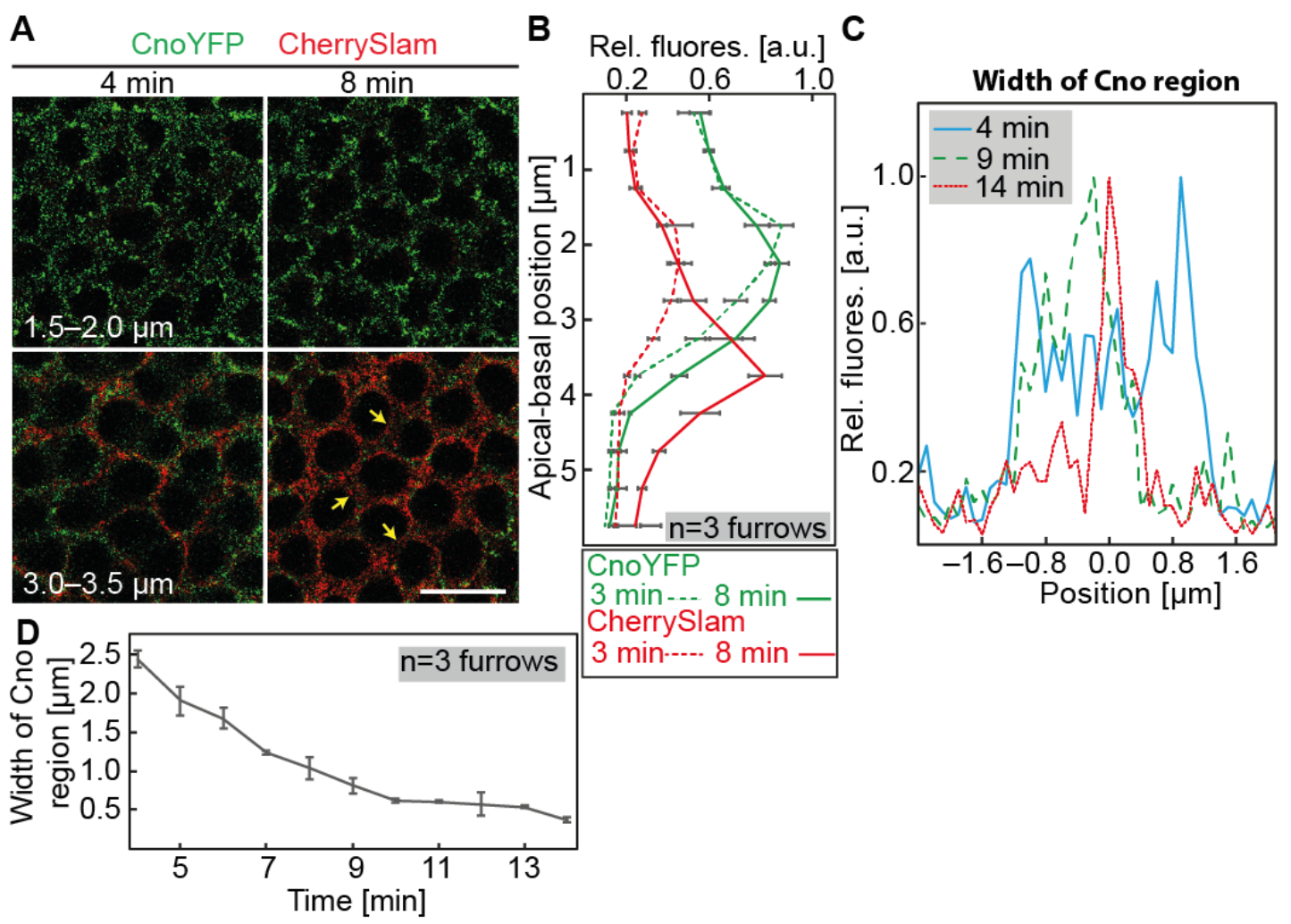

Figure 8 Dynamics of Canoe and Slam at onset of cellularization.

(A) Images from time laps recording of embryos expressing CanoeYFP (green) and CherrySlam (red) at two different axial positions indicated on lower left and two timepoints during onset of cellularization. Yellow arrowheads point to "new" furrows. (B) Relative fluorescence intensity of CanoeYFP (green) and CherrySlam (red) at "new" furrows measured along the apical-basal axis at indicated times. (C) Distribution of CnoYFP (relative fluorescence) across a "new" furrow measured at indicated timepoints. (D) Width of CanoeYFP signal in $\mu \mathrm{m}$ measured at indicated times at three "new" furrows. Error bars represent SEM. Scale bar $10 \mu \mathrm{m}$.

As the CanoeYFP signal also changed during the first minutes of cellularization from a broader distribution to a sharp subapical signal (Figure 7, Figure 8A), I quantified the signal distribution along a new furrow by measuring the fluorescence intensity and plotted it against the relative position left and right to the furrow, with zero marking the middle of the new furrow (Figure 8C). At 4 min (blue line), the signal indeed showed a broad 
distribution of around $1.2 \mu \mathrm{m}$ left and right to the middle of the new furrow, the signal already got sharper at 9 min (green dashed line) with a clear peak in the middle of the new furrow $(0 \mu \mathrm{m})$ but with some remaining signal left to the furrow. At $14 \mathrm{~min}$ a very clear and sharp peak at $0 \mu \mathrm{m}$ could be detected (red dotted line). To show the sharpening of the Canoe signal in a more quantitative way, I measured the width of the Canoe signal at three new furrows and plotted the averages against the time (Figure $8 \mathrm{D}$ ). The signal width decreased from around 2.5 to only $0.5 \mu \mathrm{m}$ in a time of $10 \mathrm{~min}$ and then stayed stable. The sharpening of the CanoeYFP signal could be explained with the fact, that the new furrow first appears as a broad and shallow groove as shown in Figure 6A. When the furrow then further invaginates into the embryo, the membranes are pulled together and come closer to each other, so that also the subapical Canoe signal gets sharper.

\section{Scribble marks the elongating lateral domain with onset of cellularization} After investigating the formation of basal and subapical cortical domains, I also analyzed the formation of the lateral domain using the lateral marker protein Scribble tagged with a GFP. I imaged embryos expressing ScribbleGFP as explained before but with a z-step size of $1 \mu \mathrm{m}$. Figure 9 shows z-stacks along the apical-basal axis over time in which $0 \mathrm{~min}$ is defined by the emergence of new furrows in interphase 14. During the end of metaphase 13 (-1 min) ScribbleGFP marked the lateral domain of the retracting metaphase furrows. After onset of cellularization, a broad signal could be detected, marking the new furrow (0-2 min). The signal got then stronger at a more lateral position with elongation of the furrows (3-11 $\mathrm{min})$. 


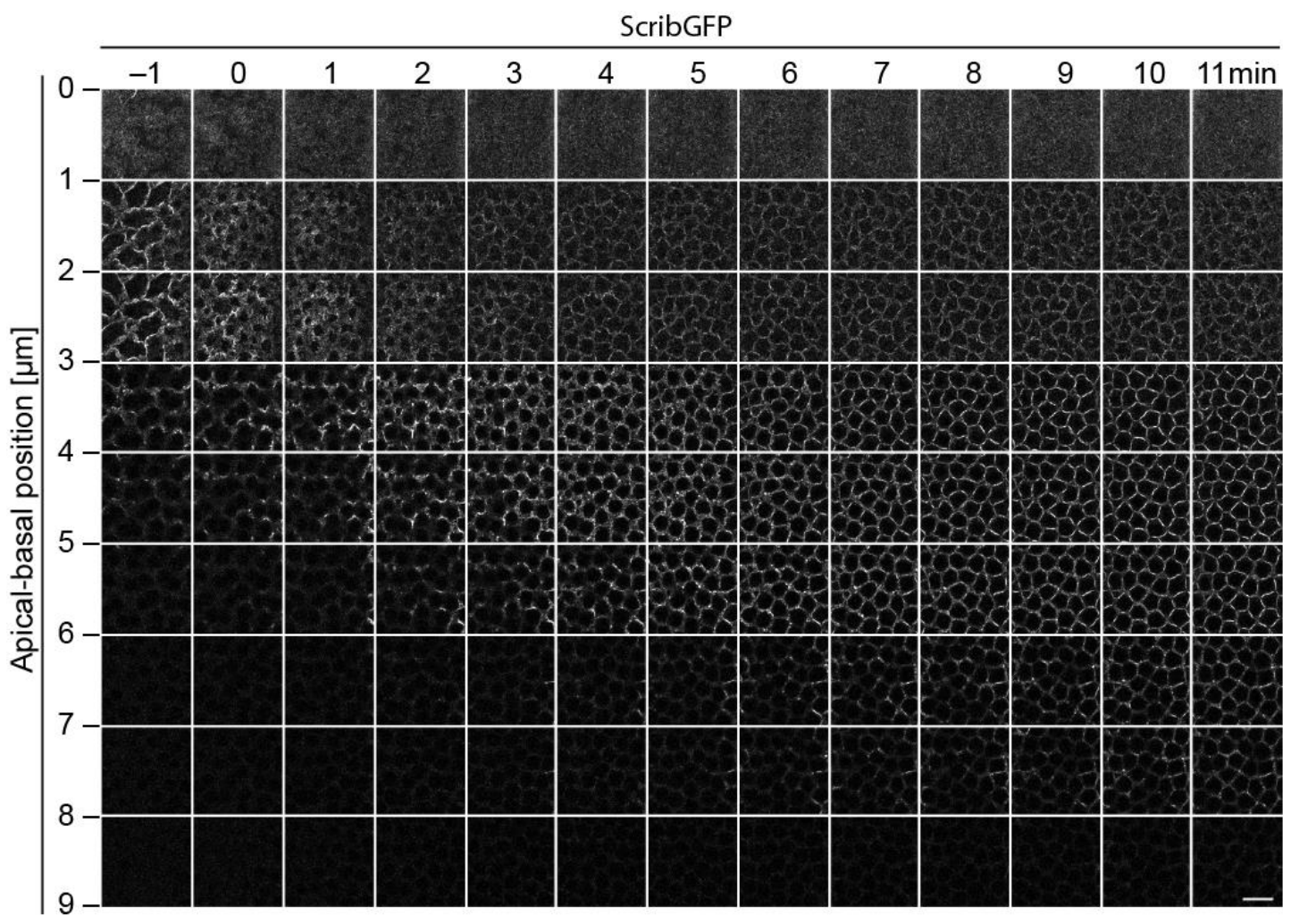

Figure 9 ScribbleGFP dynamics during mitosis 13 and interphase 14.

Images from a time lapse recording of an embryo expressing ScribbleGFP. Time from left to right, apical basal position from up to down. Scale bar $10 \mu \mathrm{m}$.

A selection of significant z-positions and timepoints is shown in Figure 10A. In the first minute of cellularization shallow grooves were visible, which were marked by a broad ScribbleGFP signal at an apical-basal position of 2-3 $\mu \mathrm{m}$. This signal got sharper at $3 \mathrm{~min}$ at a position of 3-4 $\mu \mathrm{m}$ but some broad signal was still visible, which vanished in the next minutes of cellularization. To analyze the dynamics of ScribbleGFP, I quantified the ScribbleGFP signal of three new furrows along the apical-basal axis as described before (Figure 10B). At $3 \mathrm{~min}$ (dashed line) the short furrow showed ScribbleGFP signal from 1$3 \mu \mathrm{m}$, whereas the region of the increased ScribbleGFP signal elongated with the furrow to $5 \mu \mathrm{m}$ at $8 \mathrm{~min}$ (solid line). Comparing the behavior of ScribbleGFP, CherrySlam and CanoeYFP one can conclude, that ScribbleGFP and CanoeYFP marked the new emerging furrow in contrast to CherrySlam which emerged when the new furrow elongated and formed a furrow canal. With elongation of the furrows CanoeYFP stayed at its subapical position, whereas ScribbleGFP also elongated with the furrow and marked the lateral domain. 

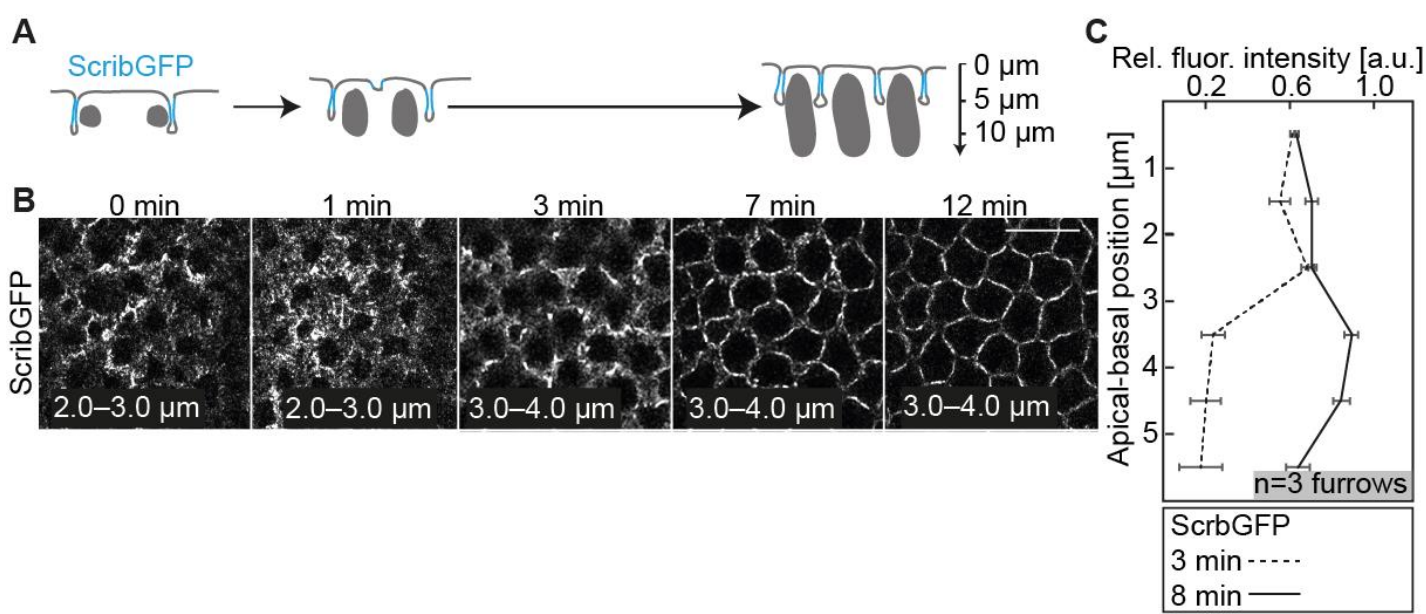

Figure 10 Dynamics of ScribbleGFP during early cellularization.

(A) Scheme for furrow formation and invagination at early cellularization. Scribble (blue) marks lateral domain. Axial (apical-basal) axis with approximate scale is indicated on the right side. (B) Images of time lapse recordings of an embryo expressing ScribbleGFP during early cellularization. Axial position is indicated. (C) Relative fluorescence intensity of ScribbleGFP at "new" furrows measured along the apical-basal axis at indicated times. Error bars represent SEM. Scale bar $10 \mu \mathrm{m}$.

\section{Subapical Canoe localization is not dependent on basal and lateral marker proteins}

To test if lateral or basal components of cortical domains affect the subapical localization and lateral restriction of Canoe, I analyzed fixed embryos mutant for scribble and slam by staining against Canoe and Dlg (Figure 11). Like in wild type embryos (Figure 11A), Canoe showed subapical enrichment and lateral exclusion in scribble mutants, although the invaginating furrows appeared weakened and Dlg spread into the apical domain (Figure 11B). Embryos mutant for slam had no or only short cellularization furrows as shown in Figure 11C. The maternal and zygotic mutation of slam was confirmed by antibody staining against Slam protein (not shown). However, also in these embryos Canoe was excluded from the lateral domain and showed subapical enrichment, although not as strong as in wild type embryos. Taken together, subapical Canoe localization was not dependent on basal and lateral markers slam and scribble. 


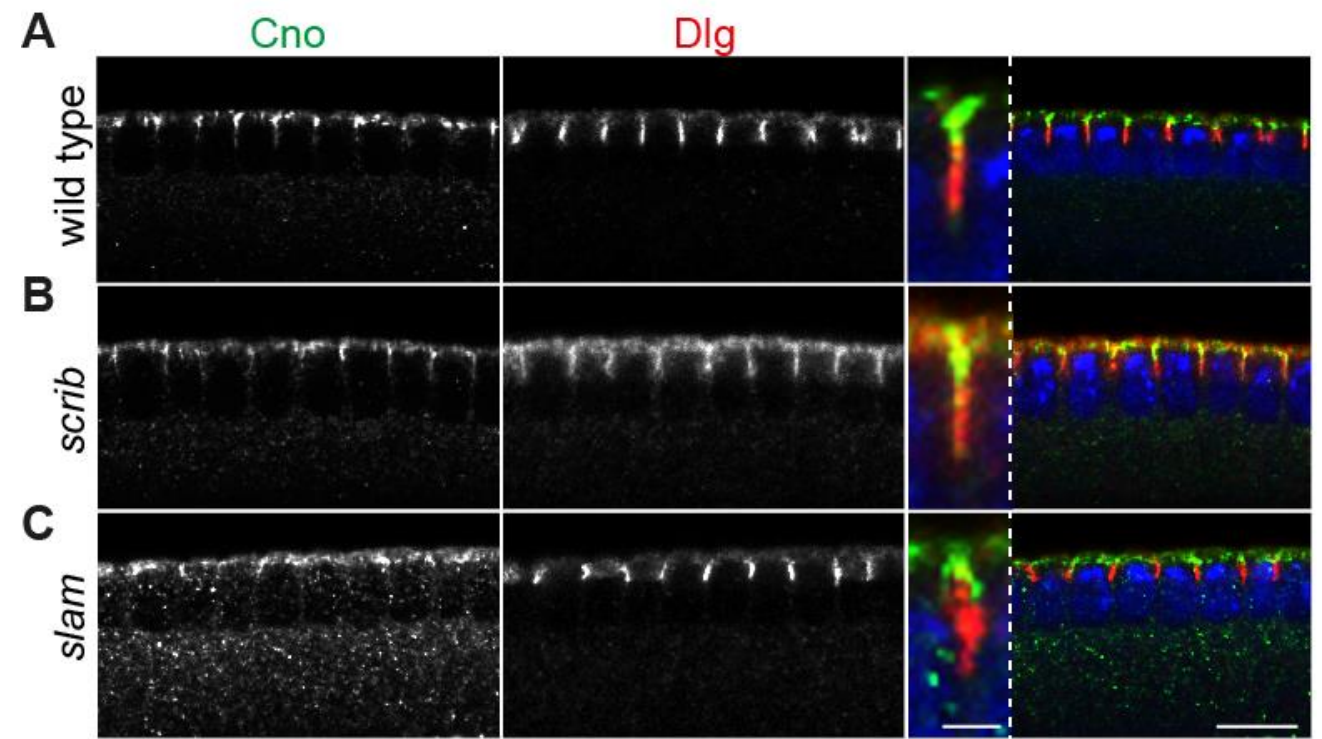

Figure 11 Genetic control of subapical Canoe by lateral and basal factors.

Images of fixed embryos in early cellularization stained for Canoe (grey/green), Dlg (grey/red) and DNA (blue). Merged images are shown in right panel. Inserts show zoom in of one furrow. Genotypes are (A) wild type, embryos from germline clones for (B) scribble and (C) slam. Scale bar $10 \mu \mathrm{m}$, insets $2 \mu \mathrm{m}$.

\subsection{The unconventional GEF ELMO-Sponge complex controls subapical Canoe localization}

The localization of the subapical proteins Canoe and Bazooka and the mechanism for positioning adherens junctions at onset of gastrulation was intensively studied in the past years. Although it is known, that the GTPase Rap1 is responsible for the subapical restriction of Canoe (Choi et al., 2013; Sawyer et al., 2009), Rap1 does not show a specific subapical localization or enrichment but is found all over the membrane during late cellularization (Sawyer et al., 2009).

\section{Rap1 controls Canoe localization but is not activated by Dizzy}

As the localization of Rap1 was only tested during mid- and late cellularization, I imaged embryos expressing GFP-Rap1 controlled by its endogenous promoter during different stages of cellularization (Figure 12). During early (5 min), mid- (35 min) and late (50 min) cellularization, GFPRap1 could be detected along the whole cellularization furrows and even along the apical membrane and no enrichment in any cortical domain was visible. Thus, I could confirm, that Rap1 shows no specific localization or enrichment also in early cellularization when the new subapical domain was formed. 


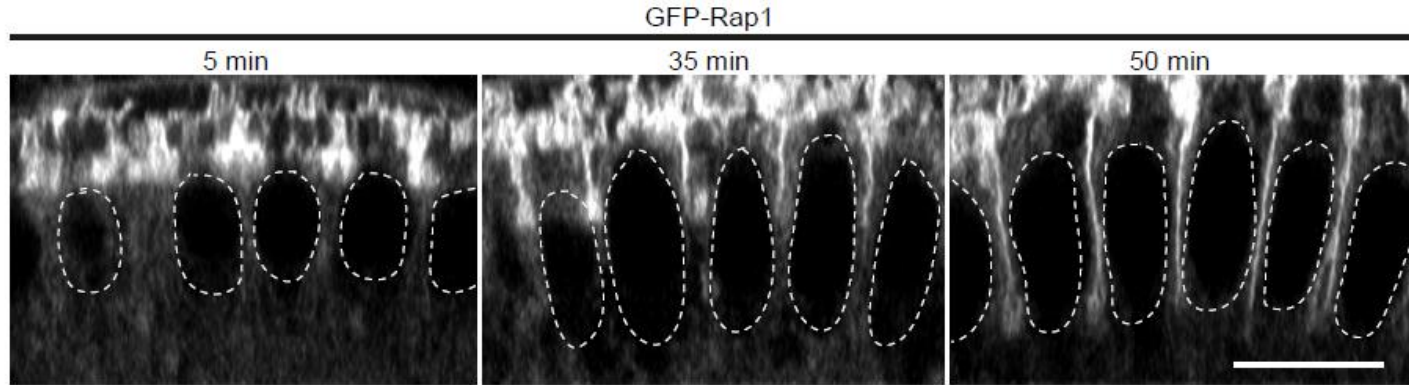

Figure 12 Dynamics of GFP-Rap1 during cellularization.

Living embryos expressing GFP-Rap1 were imaged by conducting z-stacks from the top with a step size of $0.4 \mu \mathrm{m}$ in an interval of $5 \mathrm{~min}$. Shown are orthogonal views during early ( $5 \mathrm{~min}$, left), mid (35 min, middle) and late (50 min, right) cellularization. The nuclei are indicated by dashed lines. Scale bar $10 \mu \mathrm{m}$.

To confirm, that Rap1 is also needed for Canoe localization during early cellularization, I stained fixed wild type embryos and Rap1 germ line clones against Canoe and Dlg, which was used to mark the lateral domain (Figure 13A, B). In early cellularization, Canoe localized to the subapical region and was restricted from the lateral membrane in wild type embryos (Figure 13A), whereas Canoe did not show any membrane localization at all in Rap1 mutants (Figure 13B) according to findings before (Sawyer et al., 2009).
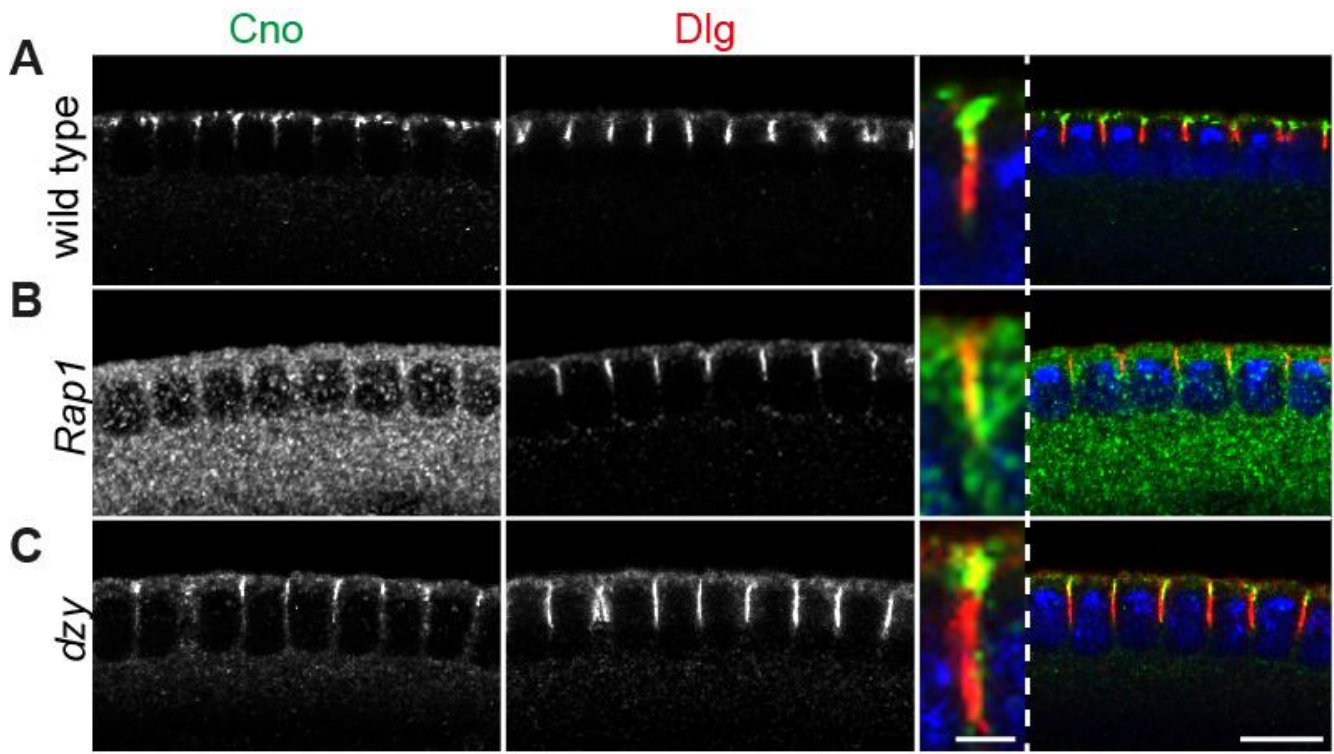

Figure 13 Genetic control of subapical Canoe by potential upstream factors.

Images of fixed embryos in early cellularization stained for Canoe (grey/green), Dlg (grey/red) and DNA (blue). Merged images are shown in right panel, insets show zoom in of one furrow. Genotypes (A) wild type, embryos from germline clones for (B) Rap1 and (C) $d z y$. Scale bars $10 \mu \mathrm{m}$, insets $2 \mu \mathrm{m}$.

As Rap1 showed no subapical enrichment it was likely that it is activated locally by a yet unknown GEF. One possible candidate for local Rap1 
activation was the GEF Dizzy, which also acts on Rap1 during gastrulation (Spahn et al., 2012). To analyze if Dizzy plays a role for Canoe activation, I stained fixed embryos maternally mutant for dizzy against Canoe and Dlg (Figure 13C). The restriction of Canoe from the lateral and apical domain and its subapical localization was not affected in dizzy mutant embryos confirming that Dizzy is not responsible for local activation of Rap1.

\section{The unconventional GEF ELMO-Sponge affects early embryogenesis and localization of Canoe during cellularization}

Several GEFs for the activation of Rap1 are known to play diverse roles during the development of various organs in Drosophila morphogenesis. The described GEFs and one GAP are depicted in Figure 14. The well characterized GEF Dizzy does not only play a role in ventral furrow formation, as its depletion led to delayed formation of adherens junctions and therefore defective furrow formation, its downregulation is also needed for normal mesoderm formation (Spahn et al., 2012). Although I could show, that Dizzy did not act on Canoe localization during early cellularization, it is needed for the localization of Canoe to tricellular junctions during the end of cellularization and by this also explains the delayed formation of adherens junctions in $d z y$ mutants (Bonello et al., 2018). Next to Dizzy, the Rap1 GEF C3G is expressed in developing muscles in Drosophila embryos and has a function in the development of body wall muscles in larvae, acting by Rap1 on integrins (Shirinian et al., 2010). Furthermore, the Rap1GEF EPAC is responsible for cAMP regulated fluid secretion in different cell types in Malpighian tubes (Efetova et al., 2013). As shown in Figure 14A, these proteins share a C-terminal GEF domain and Ras-association domains/Ras exchange motifs, which are needed for the activation of Rap1 (Efetova et al., 2013; Lee et al., 2002; Shirinian et al., 2010). Furthermore, Dizzy and EPAC share Nterminal cyclic nucleotide binding domains, meaning that they can be activated by cAMP, for example.

Figure 14B depicts the domain structure of an unconventional Rap1 GEF, which consists of the two proteins ELMO and Sponge that activate Rap1 in a complex. In this complex, ELMO provides the membrane-binding PH-domain at its C-terminus. The function of the ELMO domain is not known. With its $\mathrm{N}$ terminal SH3 domain, Sponge binds to the proline-rich C-terminus of ELMO. The GEF activity of Sponge is mediated by its DHR domains (Yajnik et al., 2003). The ELMO-Sponge complex is known to play a role in axonal patterning 
and heart development during Drosophila embryogenesis by acting on the actin cytoskeleton via Rap1 (Biersmith et al., 2011, 2015). Furthermore, it was shown, that maternal mutations of sponge and ELMO disrupt the formation of actin caps in syncytial blastoderm embryos and that these mutations are lethal during cellularization (Postner et al., 1992; Schmidt et al., 2018; Winkler et al., 2015). The domain structure of the mammalian homologue of the only known Rap1 GAP in Drosophila is depicted in Figure 14C. It was shown that RapGAP1 regulates Rap1 during posterior dorsal fold formation in embryogenesis, leading to uncoupling of actin from the junctions and by this deeper invagination than the anterior fold where RapGAP1 is not expressed (Wang et al., 2013).

For none of the conventional GEFs a function during early cellularization was described and I could also show, that Dizzy does not function on Canoe localization during early cellularization. However, as maternal mutations of both ELMO and sponge lead to phenotypes during syncytial blastoderm and cellularization, I further analyzed the functions of these proteins.

\section{AConventional Rap1 GEFs}

\begin{tabular}{|c|c|c|c|c|c|c|c|}
\hline Dzy & cNMP & REM & PDZ & & RA & GEF & 1573 аa \\
\hline C3G & & & & REM & & RasGEF & 1571 aа \\
\hline $\begin{array}{l}\text { Epac2 } \\
\text { (murine) }\end{array}$ & CNB & DEP & CNB & REM & RA & GEF & 011 aа \\
\hline
\end{tabular}

BUnconventional Rap1 GEF

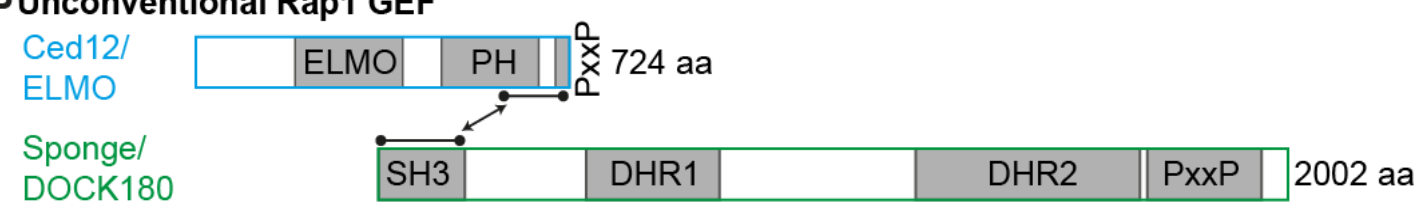

CRap1 GAP

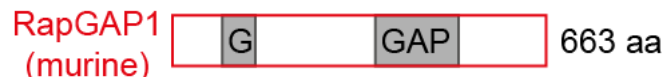

Figure 14 GEFs and GAP for Rap1.

(A) Scheme of domain structure of conventional GEFs Dizzy (Spahn et al., 2012), C3G (Shirinian et al., 2010) and Epac2 (Efetova et al., 2013) known to activate Rap1. (B) Domain structure of the unconventional Rap1 and Rac GEF ELMO-Sponge (Biersmith et al., 2011; Yajnik et al., 2003). The interaction domain is marked with a line. (C) Murine domain structure of the sole known Rap1 GAP in Drosophila (Wang et al., 2013). Domains are as follows: cNMP: cyclic nucleotide monophosphate binding domain; REM: Ras exchange motif; PDZ: PDZdomain; RA: Ras-associating domain; GEF: Guanine nucleotide exchange factor domain; RasGEF: Ras guanine nucleotide exchange factor domain; CNB: cyclic (AMP or GMP) nucleotide binding domain; DEP: Dishevelled-Egl10-pleckstrin domain; ELMO: ELMO domain; PH: Pleckstrin homology domain; SH3: SRC homology 3 domain; DHR: Dock homology region; PxxP: Proline rich region; G: GoLoco interaction motif; GAP: GTPaseactivating protein domain. Schemes show $\mathrm{N}$-termini to C-termini from left to right. 
To clarify if the ELMO-Sponge complex may be responsible for the local activation of Rap1 during cellularization, I analyzed $E L M O^{367}$ germline clones, in the following named as ELMO mutants. The ELMO ${ }^{367}$ allele was generated in an EMS screen (Luschnig et al., 2004) and mapped and characterized by Dr. Zhiyi Lv. Embryos mutant for ELMO, did not form actin caps during blastoderm interphases but had an unstructured actin cortex (Figure 15A, images made by Dr. Zhiyi Lv), furthermore cellularization did only occur at the terminal poles of the embryos as shown by staining of fixed ELMO mutant embryos against Dlg, Slam and DNA (Figure 15B, images made by Dr. Zhiyi $\mathrm{Lv})$.
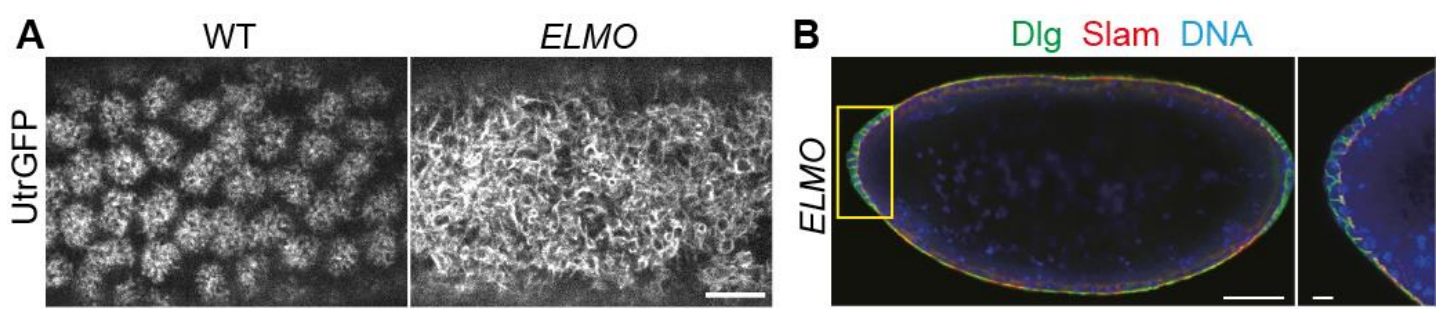

Figure 15 Blastoderm phenotype of embryos from ELMO germline clones.

(A) Images of wild type and ELMO embryos expressing the F-actin marker UtrGFP. (B) Image of fixed ELMO embryo stained for Dlg (green), Slam (red) and DNA (blue). Cellularizing terminus marked by yellow rectangle and magnified in inset. Scale bar $10 \mu \mathrm{m}$ in A and inset of $\mathrm{B}, 50 \mu \mathrm{m}$ in B. Images were made by Dr. Zhiyi Lv

As ELMO mutants did not form the two cortical domains with cap and intercap during syncytial blastoderm stage, I further analyzed if also the formation of the subapical domain was defective during onset of cellularization. For this I made use of ELMO and sponge mutant embryos to stain them against Canoe and Dlg (Figure 16). Sponge mutants resembled the phenotype with missing actin caps during syncytial blastoderm stage and only terminal cellularization (Postner et al., 1992) as described for ELMO mutants. In ELMO mutants Canoe localization was indeed perturbed as Canoe spread along the whole lateral and partially basal domains (Figure 16B). This phenotype was also visible in sponge mutants, even in late cellularization (Figure 16C). To quantify the effects of ELMO on Canoe localization, I measured the fluorescence intensity of the Canoe signal along the apicallateral membrane in in total nine cellularization furrows of three wild type and ELMO embryos and normalized the data to its peak to be able to compare intensities (Figure 16D). All measured furrows were axially aligned to their peaks which was defined as $0 \mu \mathrm{m}$. Each measured furrow is represented in the heatmaps (left panel) for wild type and ELMO mutants. In wildtype the 
highest intensities were present at the subapical domain, around $0 \mu \mathrm{m}$ along the apical-basal axis, whereas there was only weak signal detectable along the lateral domain. In ELMO mutants, a peak was still detectable at the subapical region, however also high intensities along the lateral domain were present. This was also shown by averaging the intensities measured along each membrane and plotted against the apical-basal position (Figure 16D, right panel). Taken together, it is likely that the unconventional GEF ELMO-Sponge is responsible for local activation of Rap1 to position Canoe and define the subapical domain at onset of cellularization, as Canoe localization is perturbed in ELMO and Sponge mutants.
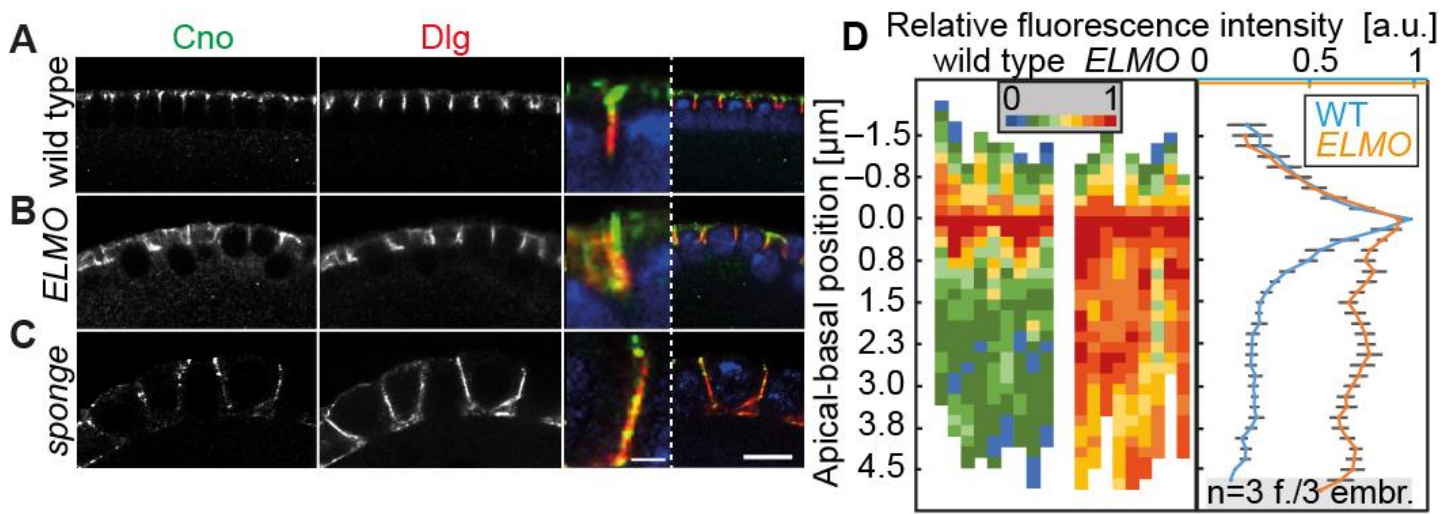

Figure 16 ELMO and Sponge are required for subapical restriction of Canoe.

(A-C) Images of fixed (A) wild type, (B) ELMO and (C) sponge embryos stained for Canoe (grey/green), Dlg (grey/red) and DNA (blue). Merged images are shown in right panel with a furrow in higher magnification in insets. (D) Heat maps and averaged values of relative fluorescence intensity along apical-basal axis aligned to the peak value measured for 9 furrows in 3 embryos. Error bars represent SEM. Scale bars $10 \mu \mathrm{m}$, insets $2 \mu \mathrm{m}$.

\subsection{ELMO-Sponge localization changes from disc-like to ring- like with onset of cellularization}

\section{Sponge localizes to actin caps, metaphase furrows and subapical domains of cellularization furrows}

The ELMO-Sponge complex as an unconventional Rap1 GEF provided a reasonable explanation for localization of Canoe and its downstream factors. As I could already show the functional relevance of ELMO-Sponge I wanted to analyze if ELMO and Sponge also showed a subapical localization during cellularization. To analyze this aspect, I stained fixed wild type embryos for Sponge (Figure 17) as its localization during early embryogenesis was not described yet. Figure 17 shows Sponge staining during different nuclear cycles in sagittal and top views. During interphases of syncytial blastoderm embryos, 
Sponge was localized at the actin caps but not at intercap regions (Figure 17, interphase 11).

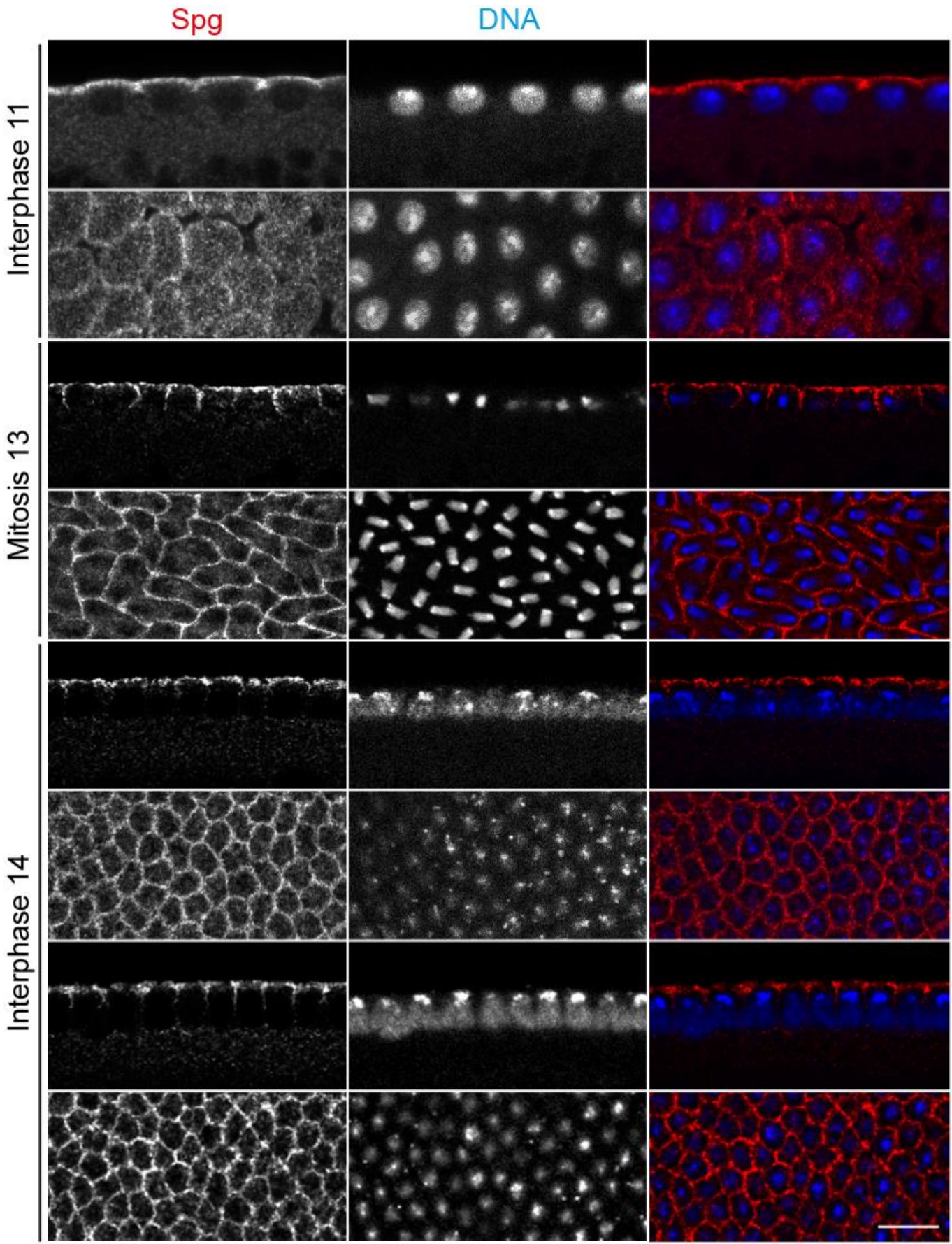

Figure 17 Sponge localization during early embryonic development.

Images of fixed wild type embryos at indicated stages stained for Sponge (grey/red) and DNA (blue). Sagittal and planar views. Scale bar $10 \mu \mathrm{m}$.

During mitosis, Sponge localized to the lateral domain of the metaphase furrow and was also found at the apical domain (Figure 17, mitosis 13). Also, at onset of cellularization in interphase 14, before nuclear elongation and with still very short furrows, Sponge was found at the actin caps with some 
enrichment at the rims of the caps, as the top view shows (Figure 17, interphase 14). With start of nuclear elongation, which still resembles early cellularization, Sponge reorganized and was enriched at the newly introduced subapical domain, which is shown in the side view (Figure 17, interphase 14). The top views of interphase 14 show a more ring like distribution of Sponge compared to its localization in interphase 11, during which the whole actin cap was stained resembling a disc-like localization. From the localization of Sponge, it is possible that it could activate Rap1 locally at the subapical domain at onset of cellularization.

\section{Sponge localization depends on ELMO but not on Rap1, Canoe and Scribble}

As described before, Sponge and ELMO build a complex in which ELMO is responsible for membrane binding (Côté et al., 2005). To test if ELMO is necessary for Sponge localization at the membrane in vivo, I stained fixed wild type and ELMO mutant embryos against Sponge and Dlg (Figure 18). Compared to the subapical Sponge enrichment in wild type embryos during early cellularization (Figure 18A), membrane localization of Sponge was indeed completely absent in ELMO mutant embryos (Figure 18B) showing, that ELMO is needed for membrane targeting of Sponge.
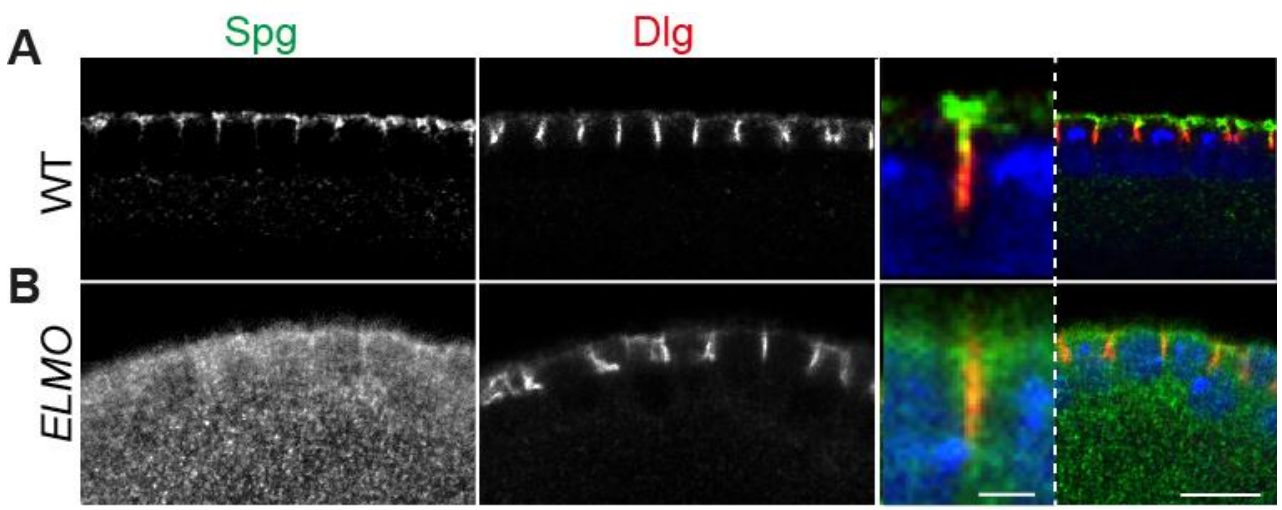

Figure 18 Membrane localization of Sponge is mediated by ELMO.

Images of fixed (A) wild type and (B) ELMO embryos in early cellularization stained for Sponge (grey/green), Dlg (grey/red) and DNA (blue). Merged images are shown in red panel with a furrow in higher magnification in inset. Scale bar $10 \mu \mathrm{m}$, insets $2 \mu \mathrm{m}$.

Furthermore, I tested if Sponge localization is affected by its downstream factors Rap1 and Canoe or by the lateral factor Scribble by staining of Sponge in the associated mutant situations (Figure 19). In Rap1 mutant embryos, Sponge enrichment was detected in the subapical region and did not spread into the lateral domain (Figure 19B), although it is possible that Sponge was 
not well restricted from the apical domain compared to wild type (Figure 19A). Also, in canoe mutants, Sponge was subapically enriched and did not spread into the lateral domain (Figure 19D). This was also the case for scribble mutants, in which Sponge enrichment was visible at the subapical domain and Sponge did not spread into the lateral domain (Figure 19C). Taken together, the subapical enrichment of Sponge was dependent on its interactor ELMO in vivo, but not on its downstream factors Rap1 and Canoe as well as on the lateral domain protein Scribble.

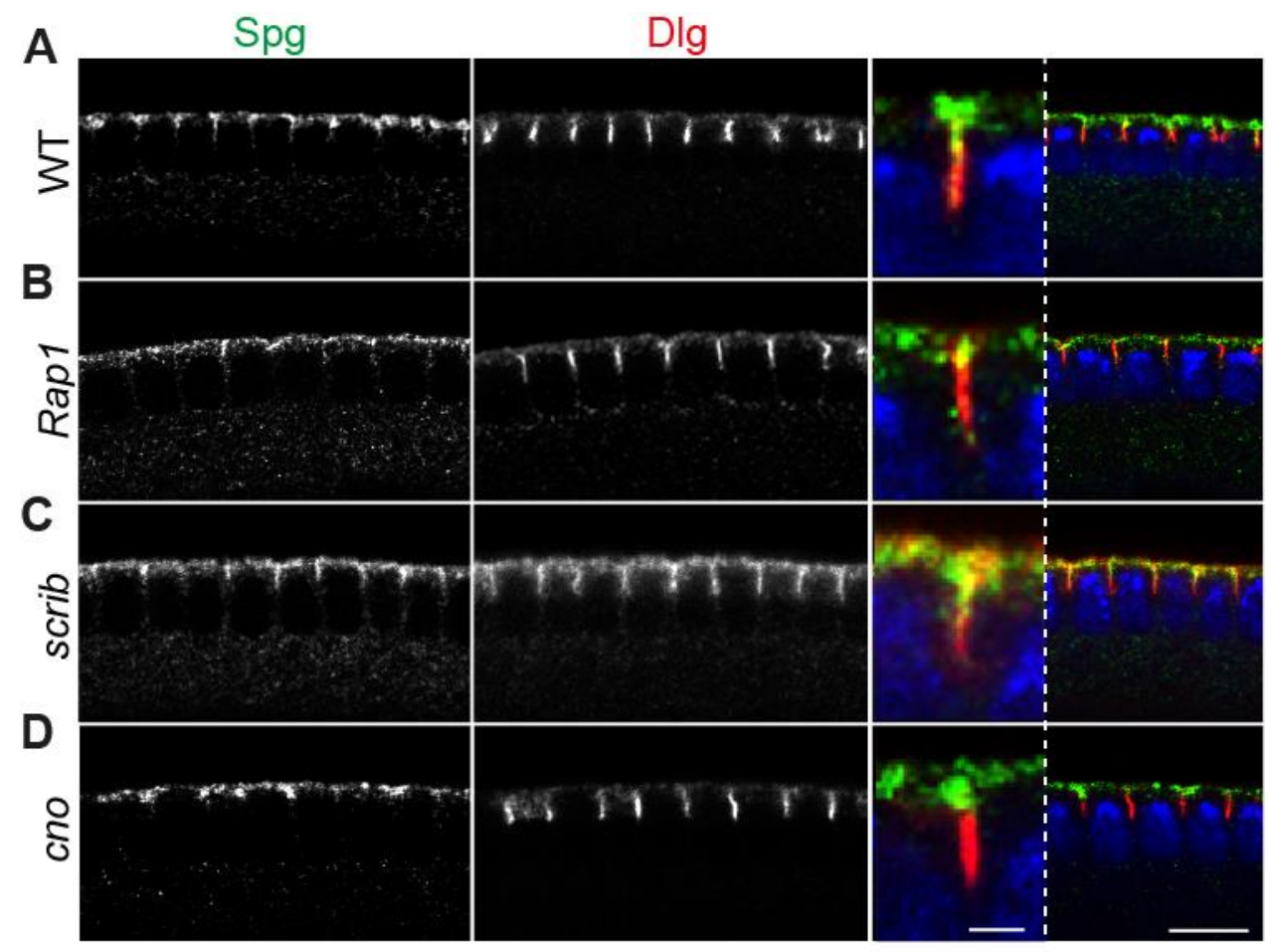

Figure 19 Genetic control of subapical Sponge.

Images of fixed embryos in early cellularization stained for Sponge (grey/green), Dlg (grey/red) and DNA (blue). Merged images are shown in right panel, inserts show zoom in of one furrow. Genotypes (A) wild type, embryos from germline clones for (B) Rap1, (C) scribble, (D) canoe. Scale bar $10 \mu \mathrm{m}$, insets $2 \mu \mathrm{m}$.

\section{ELMO shows a ring-like localization at onset of cellularization marking the position of the newly forming subapical domain}

To follow the dynamics of ELMO-Sponge in vivo, I made use of a transgenic line expressing GFP-tagged ELMO under the control of its own promoter. The transgene was made by Dr. Zhiyi Lv. Furthermore, I used CherrySlam as a marker for the basal domain. Imaging of living embryos expressing ELMOGFP and CherrySlam was done from the top view with z-stacks with a step size of $0.5 \mu \mathrm{m}$ and an interval of $1 \mathrm{~min}$ during end of mitosis 13 and beginning of interphase 14 and onset of cellularization (Figure 20). Timepoint zero was 
defined by the formation of new cellularization furrows. As shown in Figure 20, ELMO-GFP localized to metaphase furrows in metaphase 13.

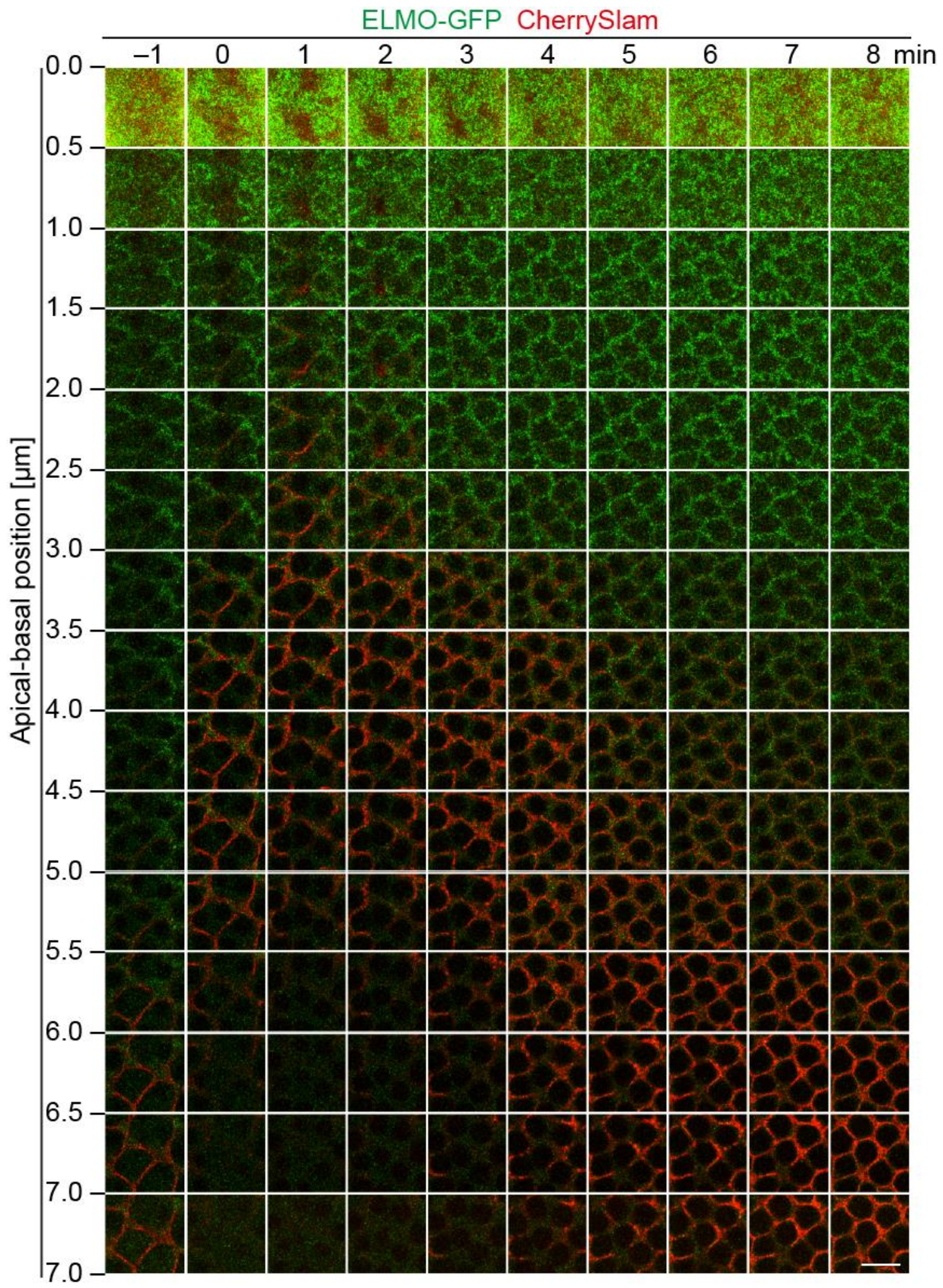

Figure 20 ELMO-GFP and CherrySlam dynamics during mitosis 13 and interphase 14.

Images of a time lapse recording of an embryo expressing Elmo-GFP (green) and CherrySlam (red). Time from left to right, apical basal position from up to down. Scale bar $10 \mu \mathrm{m}$. 
With onset of cellularization, ELMO-GFP localized to caps and showed an enrichment at the rims of the cap to form a ring-like structure, that was stable at least during the first 8 minutes of cellularization when furrows elongated, shown by basal CherrySlam signal, as indicated. Figure 21A shows ELMOGFP signal from an actin cap during an earlier interphase, that had no enrichment at the rim of the caps, forming a more disc-like localization.

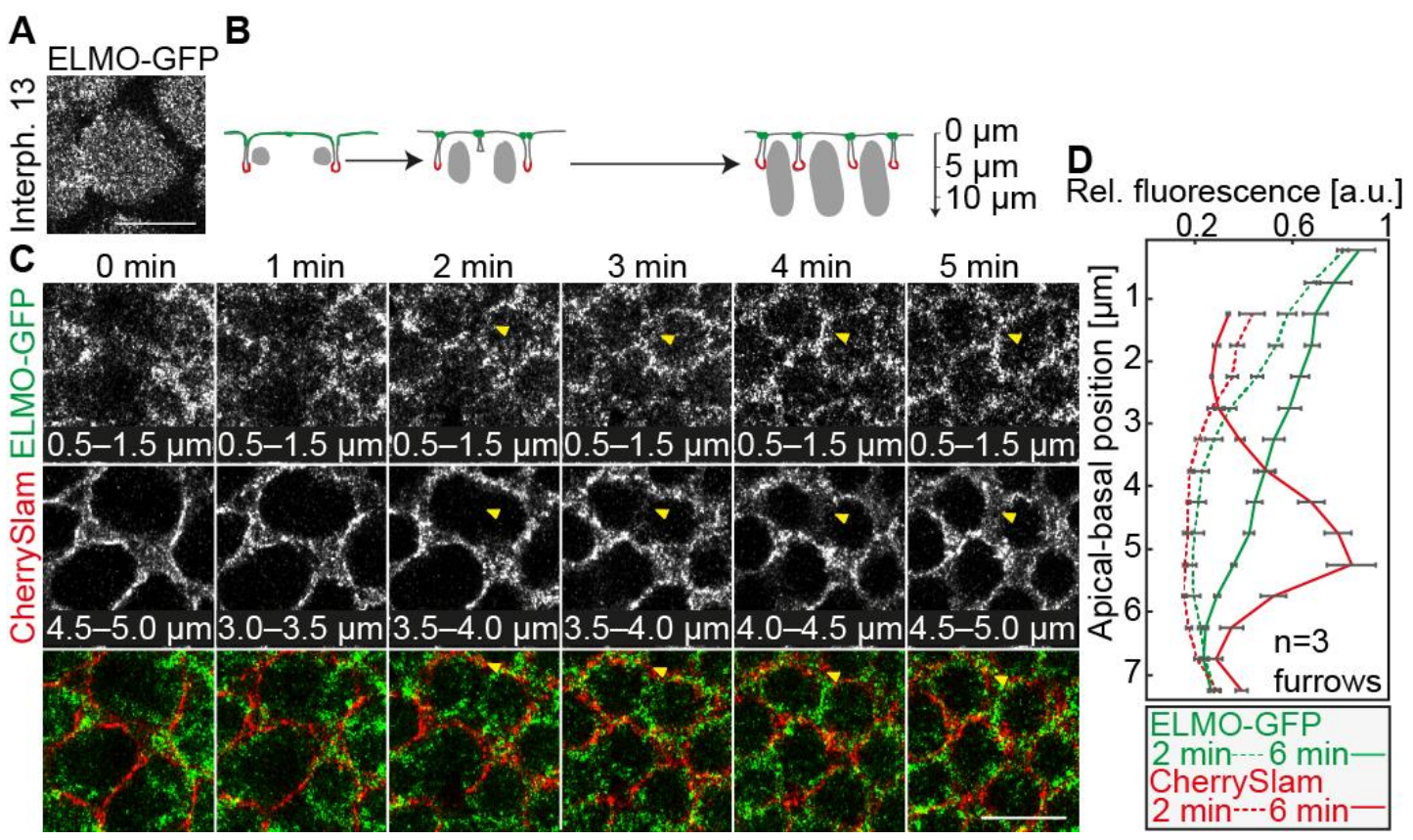

Figure 21 Dynamics of ELMO-GFP during early cellularization.

(A) Image from a time laps recording of an embryo expressing ELMO-GFP shows ELMO-GFP localization at the cap during interphase 13. (B) Scheme for furrow formation and invagination in early cellularization. Subapical and basal domains are marked in green and red, respectively. Axial (apical-basal) axis with approximate scale is indicated. (C) Images from time lapse recordings including axial stacks of embryo expressing ELMO-GFP (grey/green) and CherrySlam (grey/red) during mitosis 13 and early interphase 14. Axial position is indicated. Yellow arrowhead points to position of "new" furrows. (D) Relative fluorescence intensity of ELMO-GFP (green) and CherrySlam (red) at "new" furrows measured along the apical-basal axis at indicated times. Error bars represent SEM. Scale bar $10 \mu \mathrm{m}$.

To analyze the dynamics of ELMO-GFP in more detail I selected significant zpositions along the apical-basal axis and timepoints at onset of cellularization, which are depicted in Figure 21C, with a schematic overview of the sagittal view in Figure 21B. During interphases in syncytial blastoderm, ELMO-GFP localized to the whole cap domain in a disc like pattern (Figure 21A). At onset of interphase 14 (Figure 21C, $1 \mathrm{~min}$ ), ELMO-GFP got enriched at the rims of the actin caps at a z-position of around 0.5-1.5 $\mu \mathrm{m}$, as also described before for Sponge in fixed embryos. The new furrow was formed at the position where two rings came together as indicated by a yellow arrowhead. Similar to the 
quantifications I showed for CanoeYFP and ScribbleGFP, I measured the fluorescence intensity of ELMO-GFP and CherrySlam along the apical-basal axis of three new furrows in one embryo, normalized the data to their peaks and plotted the averages of the relative fluorescence intensity against the apical-basal position (Figure 21D). After 2 minutes, when the new furrow was still a shallow groove, revealed by the missing peak of CherrySlam intensity (dashed red line), ELMO-GFP already showed a subapical enrichment which was diminished in basal direction (dashed green line). After the formation and invagination of the new furrow at $6 \mathrm{~min}$, indicated by a peak of CherrySlam intensity at around $4.5-5.5 \mu \mathrm{m}$ (red line), ELMO-GFP intensity was still subapically enriched and decreased in direction of the basal domain (green line).

Taken together, I could show that the unconventional GEF ELMO-Sponge is a likely candidate to activate Rap1 locally to position Canoe and its downstream factors. The change of ELMO-Sponge localization from a more disc-like distribution during blastoderm interphases to the ring-like enrichment during onset of cellularization may provide an explanation for the switch from three cortical domains in metaphase and two cortical domains in interphase during syncytial blastoderm to four cortical domains in cellularization by the insertion of the new subapical domain.

\subsection{Bazooka gets enriched at the subapical domain during cellularization}

The PDZ-domain protein Bazooka is another known factor in the pathway for the upset of the subapical domain downstream of Canoe (Choi et al., 2013). To get a further insight into the formation of the subapical domain, I analyzed the localization and dynamics of Bazooka. As it is known, that Bazooka is transported to the subapical domain by Dynein transport along microtubules (Harris and Peifer, 2005) and gets enriched during cellularization, I wanted to analyze when Bazooka gets visible at the subapical domain. For this purpose, I used staged and fixed wild type embryos and stained against Bazooka and Armadillo as lateral marker and imaged with same imaging conditions (Figure 22). During mitosis 13, Bazooka was not visible at the metaphase furrows, although its localization at metaphase furrows has been described before (Harris and Peifer, 2004). This could be explained by different imaging set ups, as I wanted to compare protein localization during different stages of 
blastoderm embryos. At early cellularization, before nuclear elongation, Bazooka was still not visible at the subapical domain (Figure 22), at this stage a clear subapical localization of Canoe and Armadillo accumulation was already visible as described before. With nuclear elongation, Bazooka got enriched at the subapical domain (Figure 22, third panel), however also during mid cellularization there were still some Bazooka puncta visible at the lateral membrane (Figure 22, fourth panel). At the end of cellularization Bazooka formed then a tight subapical enrichment (Figure 22, bottom panel).

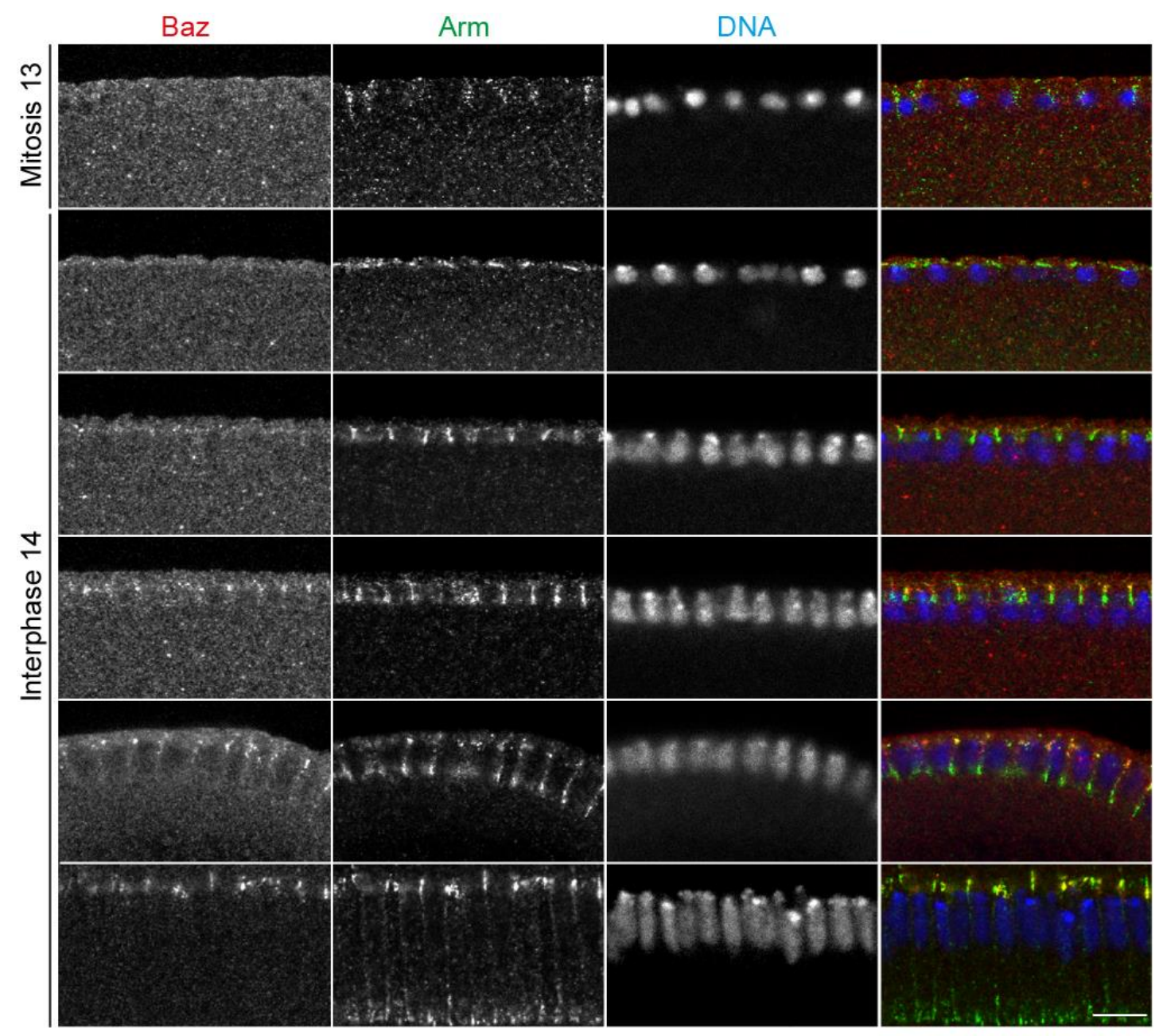

Figure 22 Subapical enrichment of Baz during cellularization in fixed embryos.

Images from wild type embryos stained for Bazooka (grey/red), Arm (grey/green) and DNA (gey/blue) during mitosis 13 and interphase 14. Images were conducted with same laser settings and processed with same brightness and contrast settings. Scale bar $10 \mu \mathrm{m}$.

I also detected BazookaGFP signal in living embryos. For this I made use of a fly line, which expressed Bazooka tagged with a GFP at the endogenous locus. Imaging of side views using a confocal laser scanning microscope was not possible as focusing around $75 \mu \mathrm{m}$ into the embryo led to non-detectable signal, even if I used the sensitive AiryScan detector. To analyze, Bazooka 
dynamics during the course of cellularization, I imaged the embryos during mitosis 13 and interphase 14 from the top view in intervals of 1 minute and made z-stacks with a step size of $0.5 \mu \mathrm{m}$. To collect all signal and visualize BazookaGFP puncta as early as possible, I summarized the z-stacks and selected several time points as shown in Figure 23. During mitosis 13, BazookaGFP puncta were only weakly detectable at the metaphase furrows, confirming the staining of the fixed embryos (Figure 23). During onset of cellularization in interphase 14, BazookaGFP puncta were not detectable (9$28 \mathrm{~min})$. The first visible puncta could be detected at a timepoint of $31 \mathrm{~min}$ after onset of cellularization, which got enriched with ongoing cellularization (34-40 min), resembling the staining of the fixed embryos shown before.

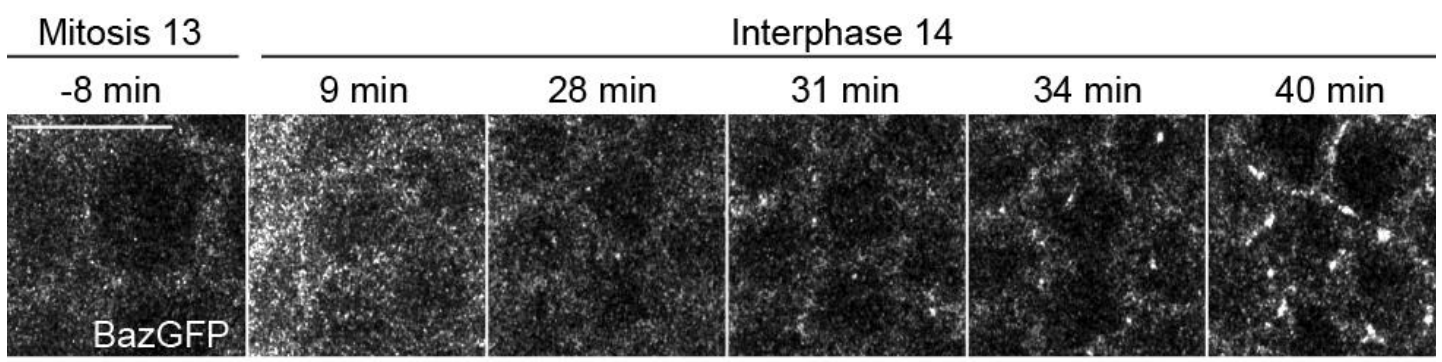

Figure 23 Subapical enrichment of Baz during cellularization in living embryos.

Summarized z-stacks from images of a movie of a wild type embryo expressing BazGFP during mitosis 13 (-8 min) and cellularization (9 min- $40 \mathrm{~min}$ ) at a room temperature of $\sim 20^{\circ} \mathrm{C}$. Accumulation of BazGFP puncta can be found at a time point of $31 \mathrm{~min}$. Scale bar $10 \mu \mathrm{m}$.

To image Bazooka dynamics in sagittal sections I made use of two-photon microscopy. By this technique the fluorophores are excited by two photons of a wavelength around $900 \mathrm{~nm}$ instead of one of $488 \mathrm{~nm}$, leading to the possibility to focus deep into the embryo. In Figure 24 BazookaGFP dynamics are shown in side views from onset of cellularization confirming the results as shown before with Bazooka getting visible around mid-cellularization (40 $\mathrm{min}$ ) and getting stronger and more enriched to the end of cellularization (60 $\mathrm{min})$ and onset of gastrulation $(80 \mathrm{~min})$.

From this data I could conclude, that Bazooka gets enriched at the subapical domain gradually during the course of cellularization with a clear subapical enrichment visible during mid-cellularization. 


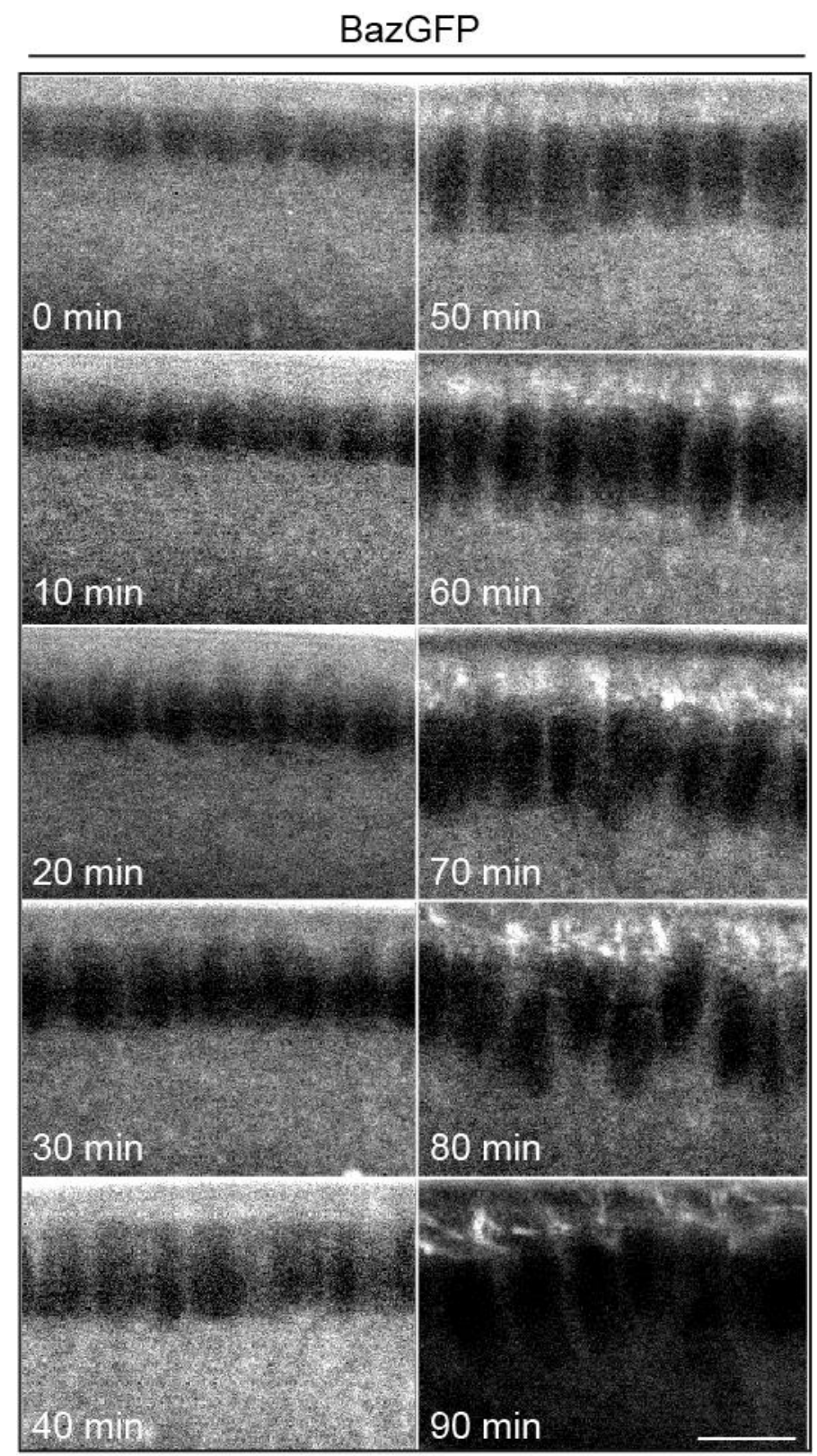

Figure 24 BazookaGFP dynamics imaged by two-photon microscopy.

Living embryos imaged with a two-photon microscope in intervals of $10 \mathrm{~min}$ and a room temperature of $\sim 20^{\circ} \mathrm{C}$. Timepoint 0 min was set by the appearance of interphase 14 nuclei. Time points are as indicated. Scale bar $10 \mu \mathrm{m}$.

\section{Localization of Bazooka and Armadillo are dependent on ELMO-Sponge}

As I investigated the role of the unconventional GEF complex ELMO-Sponge in chapters 3.2 and 3.3, I wanted to examine if ELMO-Sponge do not only play a role in formation of the subapical domain and correct localization of Canoe but also have a function on the subapical localization of Canoes downstream factors Armadillo and Bazooka. For this, I stained fixed wild type and ELMO mutant embryos and stained for Armadillo and Bazooka (Figure 25). As described before, Bazooka and Armadillo showed subapical enrichment 
during mid and late cellularization in wild type embryos (Figure 25A). Although staging of ELMO mutants was somehow vague due to their perturbed morphology, I could detect Bazooka and Armadillo staining dispersed over the whole cellularization furrow (Figure 25B). However, as I could show before, that also Canoe was mislocalized in ELMO mutants it is likely, that the effect of ELMO on Bazooka and Armadillo is more indirect and due to the mislocalization of the upstream factor Canoe.

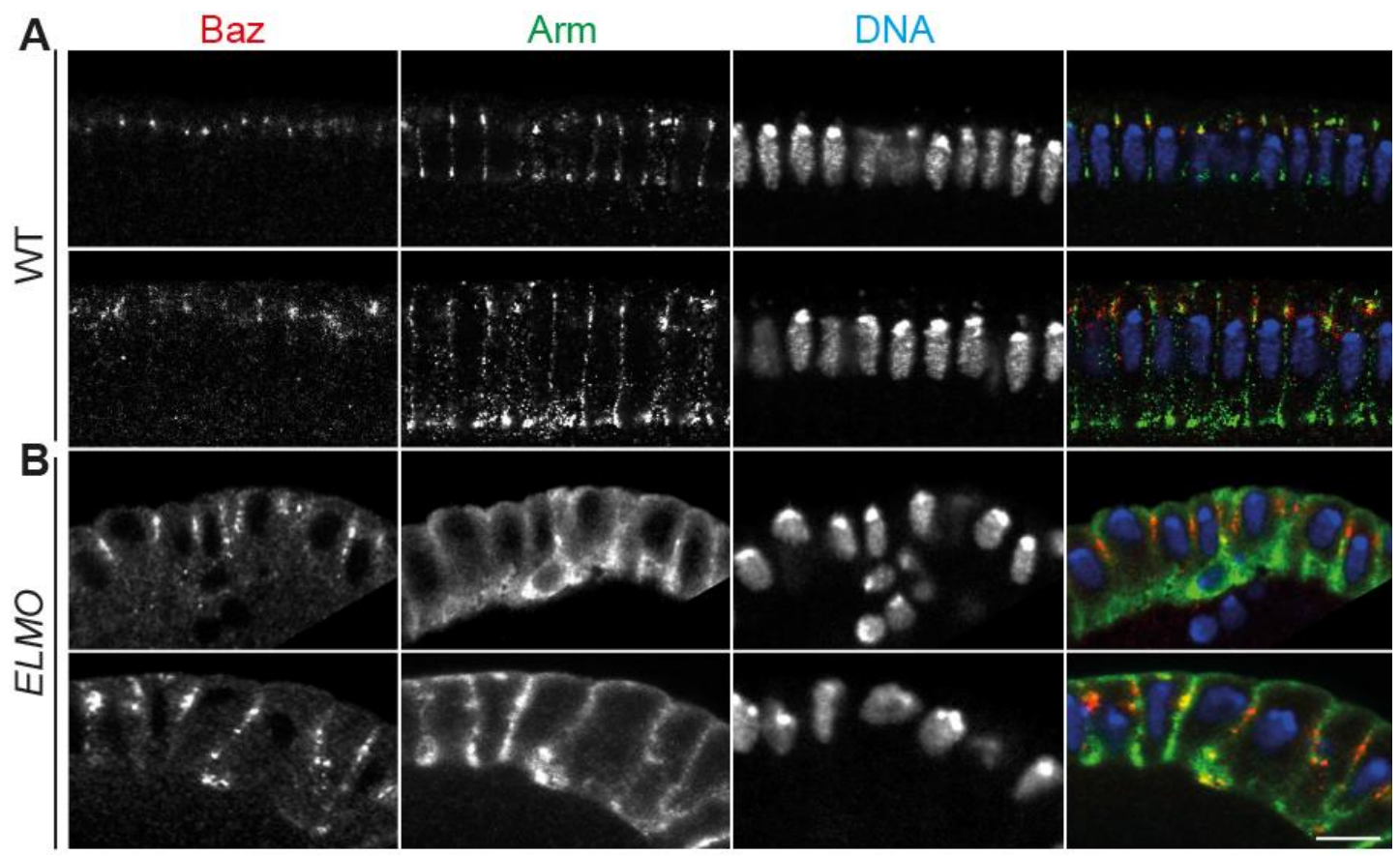

Figure 25 ELMO is required for subapical enrichment of Baz and Arm.

(A-B) Images of fixed (A) wild type and (B) ELMO embryos during mid and late cellularization stained for Baz (grey/red), Arm (grey/green) and DNA (grey/blue). Merged images are shown in right panel. Scale bar $10 \mu \mathrm{m}$.

\subsection{Bazooka is required for subapical localization of Canoe}

It was described before, that Bazooka is needed for subapical localization of Canoe during late cellularization and for its enrichment at tricellular junctions at onset of gastrulation (Choi et al., 2013). A function for Bazooka in the localization during early cellularization was not shown yet. For this purpose, I stained fixed wild type and bazooka RNAi embryos for Canoe and DNA (Figure 26). During mid-cellularization Canoe localized to the subapical region in wild type embryos that displayed a hexagonal enrichment in top views (Figure 26A). During early cellularization in bazooka RNAi embryos, the subapical Canoe enrichment was still detectable in sagittal sections, though the top view already revealed a broader Canoe enrichment (Figure 26B, upper panels). During mid-cellularization Canoe was also detectable along the 
lateral domain in sagittal sections of bazooka RNAi embryos and the top view revealed an even more dispersed Canoe signal than during early cellularization (Figure 26B, lower panels). The functionality of bazooka knock down by RNAi was shown by typical holes in the amnioserosa of stage 14 embryos (Figure 26C).

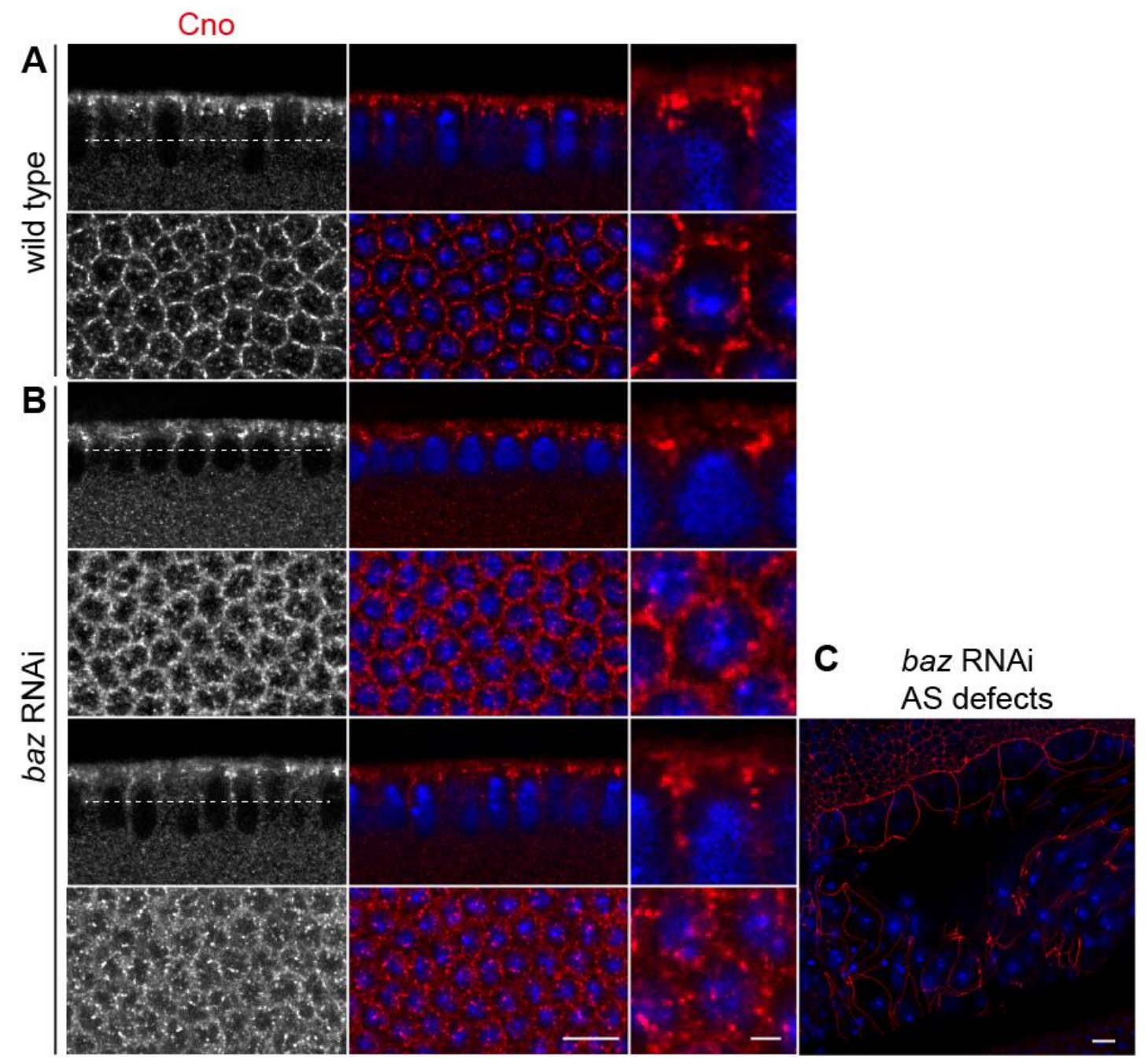

Figure 26 Baz functions in Cno localization during cellularization

(A-B) Fixed cellularizing (A) wild type and (B) baz RNAi embryos stained for Cno (grey/ red) and DNA (blue). Merged images are shown in right panels, zoom-ins in right panel. The depth of the cellularization furrow is marked by a dashed line. Sagittal views are shown in upper panels, top vies (maximum intensity projections of z-stacks) in lower panels. (C) baz RNAi embryo stained for Cno (red) and DNA (blue) with typical holes in the amnioserosa tissue. Scale bar $10 \mu \mathrm{m}$, zoom-ins $2 \mu \mathrm{m}$.

I also tested if the localization of Sponge depends on Bazooka by staining wild type and bazooka RNAi embryos against Sponge and Dlg (Figure 27). In wild type I was able to detect subapical Sponge enrichment during early cellularization in sagittal sections and also the hexagonal signal in top views (Figure 27A). Although I could detect a mislocalization of Dlg in baz RNAi 
embryos with continuous staining also at the tricellular junctions, which show reduced Dlg in wild type embryos, subapical Sponge localization was not affected in bazooka RNAi embryos (Figure 27B). From this data I could conclude, that not only Canoe affected Bazooka localization but that also Canoe was controlled by Bazooka already during early and midcellularization whereas it did not affect Sponge localization.

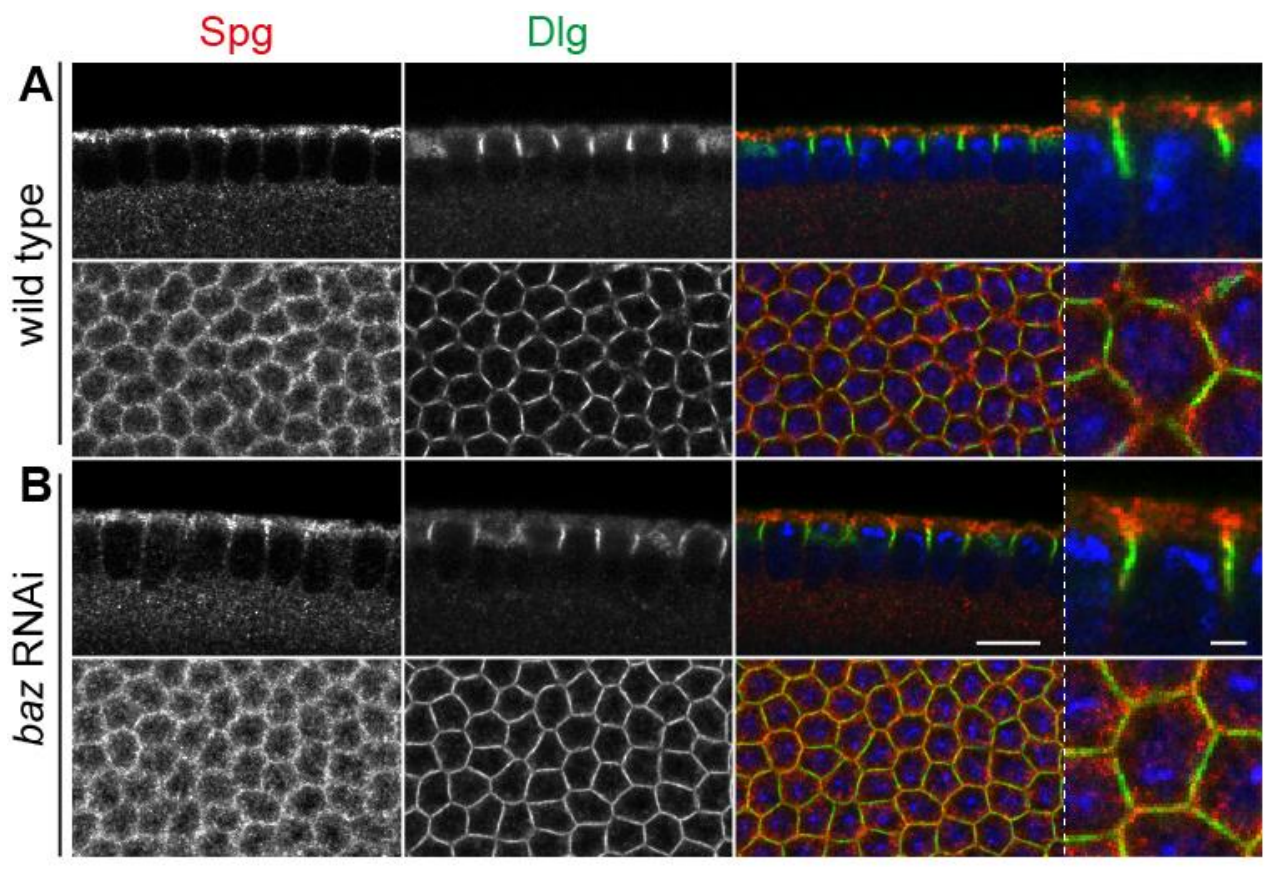

Figure 27 Spg localization is not affected by Baz.

(A-B) Fixed early cellularizing (A) wild type and (B) baz RNAi embryos stained against Spg (grey/ red), Dlg (grey/green) and DNA (blue). Merged images are shown in right panels, zoom-ins in right panel. Upper panels show sagittal sections, lower panels top view of maximum intensity projections. Scale bar $10 \mu \mathrm{m}$, zoom-ins $2 \mu \mathrm{m}$.

\subsection{The onset of zygotic gene expression is necessary for correct localization of Canoe}

To test if expression of zygotic genes is necessary for the introduction of the subapical domain during cellularization, I made use of the drug $\alpha$-amanitin, that inhibits RNA polymerase II and with this zygotic gene expression (Edgar et al., 1986). It was shown before, that the injection of $\alpha$-amanitin leads to an arrest in interphase 14 and no invagination of cellularization furrows takes place (Edgar et al., 1986).

I injected $\alpha$-amanitin in wild type embryos expressing Canoe-YFP during preblastoderm development, let them develop until interphase 13-14 and stained them after fixation to visualize Canoe, F-actin and DNA for control of 
the developmental stage (Figure 28). Figure 28A shows a non-injected embryo, that was co-stained with injected embryos. In this embryo F-actin and Canoe show the typical hexagonal localization described for onset of cellularization. A zoom in of Canoe signal and merged image are shown in Figure 28 $\mathrm{A}^{\prime}$ to point the staining up. In $\alpha$-amanitin injected embryos during interphase 14 , this hexagonal pattern of F-actin and Canoe could not be detected anymore (Figure 28B, B'). This observation confirms the hypothesis, that onset of zygotic gene expression is needed for correct localization of Canoe and formation of the subapical domain.

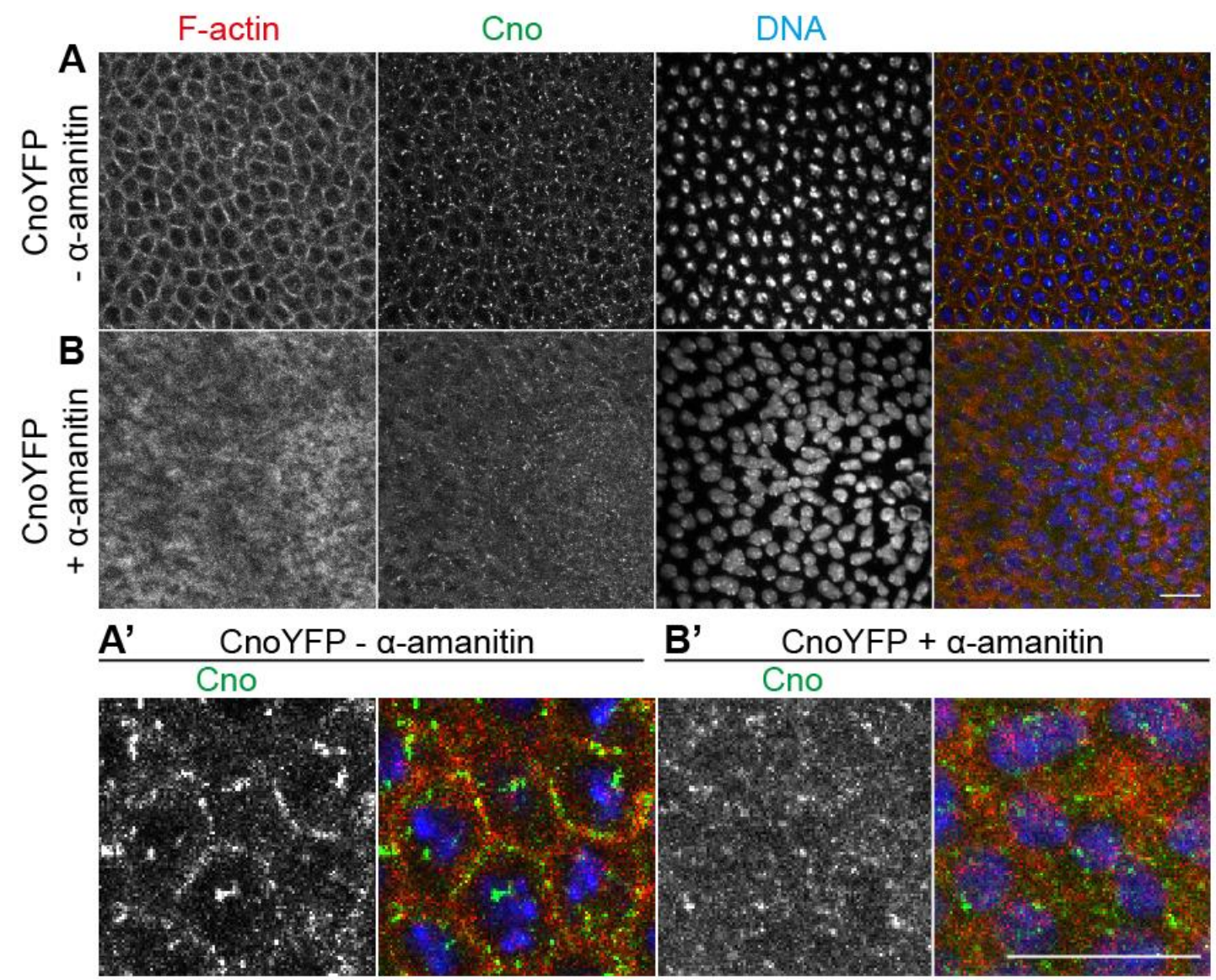

Figure 28 Zygotic gene expression is necessary for Canoe localization during cellularization.

(A-B) Fixed non-injected (A) and $\alpha$-amanitin-injected (B) embryos expressing CanoeYFP stained against F-actin (grey/ red), CanoeYFP (grey/green) and DNA (grey/ blue). Merged images are shown in right panels. (A', B' show zoom ins from (A, B). Scale bars $10 \mu \mathrm{m}$.

As a control that not the nuclear cycle number but the onset of zygotic gene expression is responsible for the correct localization of Canoe and formation of the subapical domain, I made use of two mutants that display either an increased number of cell cycles before cellularization or a decreased number of cell cycles before start of cellularization (Figure 29). 


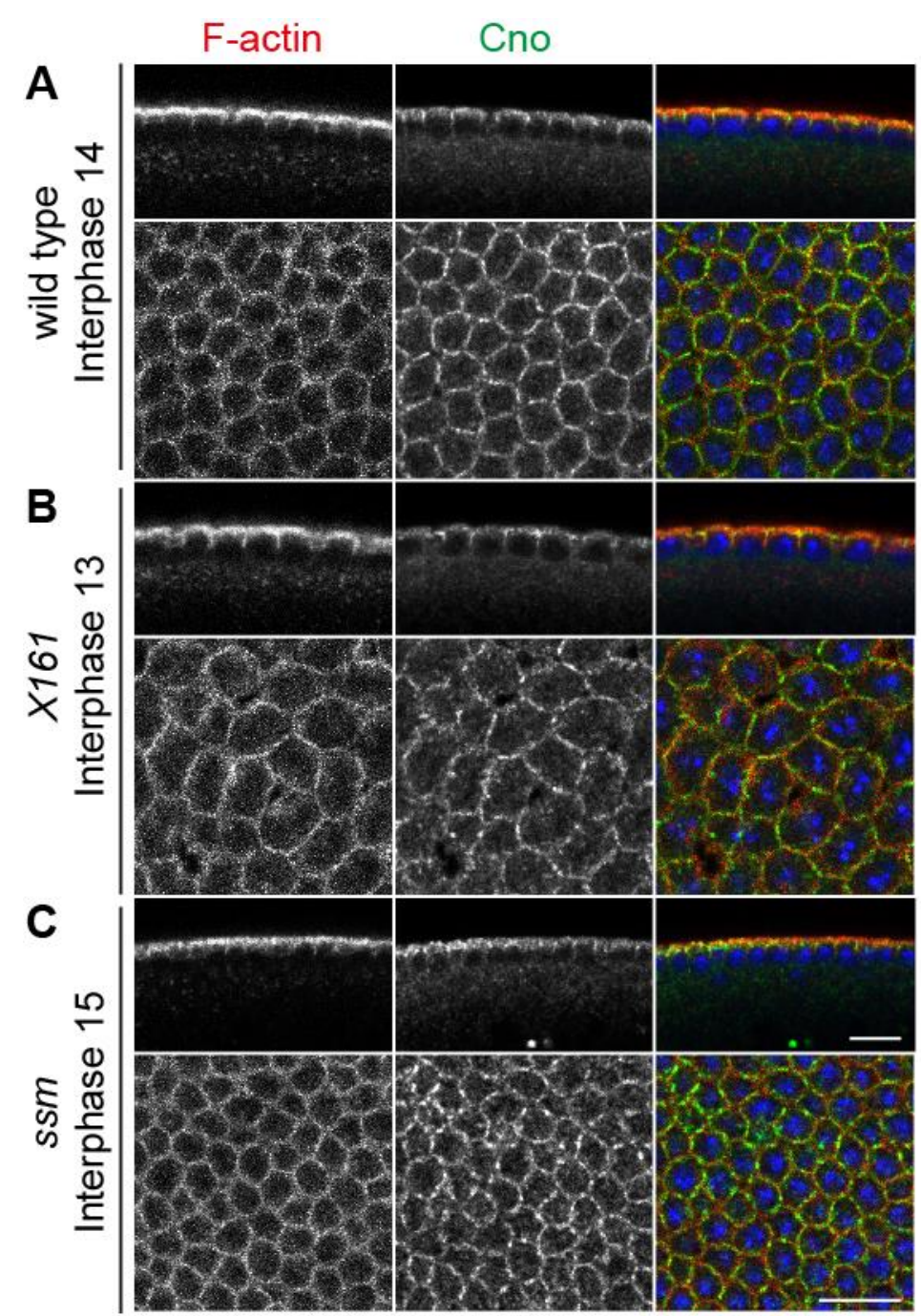

Figure 29 Cno localizes to subapical domain at onset of cellularization independently of the cell cycle number.

(A-C) Fixed (A) wild type, (B) X161 and (C) ssm embryos during early cellularization stained against Cno (grey/green), F-actin by Phalloidin (grey/red) and DNA (blue). Cell cycle number is as indicated and was defined by the number of nuclei. Merged images are shown in right panels, sagittal views in upper and top views of the subapical section in lower panels. Scale bar $10 \mu \mathrm{m}$.

I compared the localization of F-actin and Canoe in early interphase 14 wild type embryos (Figure 29A) to embryos maternally mutant for X161 (Figure 29B) which start cellularization already in interphase 13 (Sung et al., 2013) and embryos maternally mutant for sésame (ssm) (Figure 29C) which are haploid and display another nuclear cycle before they start cellularization in interphase 15 (Loppin et al., 2000). The nuclear cycle number was defined by the number of nuclei. Like in wild type, F-actin localized to the actin caps and invaginating furrows as I could show in the sagittal views and in top views at a z-position of the subapical region I could detect the typical hexagonal enrichment in wild type and both mutants. This was also the case for the 
subapical enrichment of Canoe, making clear that the formation of the subapical domain does not depend on the nuclear cycle number but on the onset of zygotic gene expression. 


\section{Discussion}

The main goal of this study was to analyze the formation of cortical domains during the generation of an epithelium. For this purpose, I made use of the progress called cellularization happening during early embryogenesis of Drosophila melanogaster development. During early embryogenesis, the Drosophila embryo shows different numbers of cortical domains, which consist of actin filaments underlying the plasma membrane, accompanied by different sets of domain-specific and domain-unspecific proteins. During preblastodermal development, only one cortical domain can be observed. During syncytial blastoderm, when the nuclei reach the embryonal periphery and perform nuclear divisions, more cortical domains are introduced. During syncytial blastoderm interphases two cortical domains can be observed, which are cap and intercap domains, whereas three domains are visible during mitosis. During mitosis, metaphase furrows reach around $10 \mu \mathrm{m}$ into the interior of the embryo, separating the spindles of the dividing nuclei from each other. The metaphase furrows consist of lateral and basal domains, whereas the apical domain underlies the plasma membrane in between the metaphase furrows. During cellularization, a fourth cortical domain is introduced between apical and lateral domain, namely the subapical domain. The proteins belonging to this domain will later help to direct proteins that make up adherens junctions to their final position in the epithelium.

The process how the PDZ-domain-protein Bazooka is directed to the subapical domain during late cellularization was researched intensely in the past years and next to binding to a cortical F-actin scaffold, also active transport along microtubules by Dynein was reported (Harris and Peifer, 2005). Furthermore, it was also described, that subapical Canoe is acting upstream of Bazooka, as its subapical localization was affected in canoe mutants and that correct Canoe localization is mediated by the GTPase Rap1 (Choi et al., 2013; Sawyer et al., 2009). However, the regulation of Rap1 was not described yet as well as the new formation of the subapical domain by subapical Canoe localization is not understood yet.

The aim of the first part of my study was to get further insight into the new domain formation. For this aspect I made use of domain specific proteins like Canoe for the subapical domain, Scribble for the lateral domain and Slam for the basal domain and analyzed their dynamics before and during onset of 
cellularization. Furthermore, I investigated the regulation of Canoe by Rap1GTPase and the local activation of the GTPase by the unconventional GEF ELMO-Sponge. Until now, the formation and introduction of the subapical domain was not studied yet as most of the studies that investigated subapical proteins were performed during mid- or late cellularization, or even during gastrulation.

In my study, I was able to show, that the subapical domain is introduced within the formation of a new cellularization furrow, to which CanoeYFP localized when it was still a groove. With invagination of the furrow, that was tracked with the basal marker CherrySlam, CanoeYFP stayed subapical and its signal was not detected at the elongating lateral domain. I was able to show the early restriction of Canoe to the subapical domain not only in movies from living embryos but was also able to quantify this behavior. In addition, fixed samples of early cellularizing embryos were used to show and quantify the subapical localization of Canoe during early cellularization.

After clarifying that Canoe was restricted to the subapical domain from onset of cellularization on, I wanted to analyze the regulation of its localization. It was shown before, that the GTPase Rap1 is upstream of Canoe and necessary for its cortical localization during mid- late cellularization (Choi et al., 2013). I was able to confirm this relationship during early cellularization, as cortical Canoe localization was completely lost in Rap1 mutants also in this stage. However, it was also described before, that Rap1 does not show any enrichment at the subapical cortical domain that could explain its role in subapical positioning of Canoe. In contrast Rap1 could be found along all cortical domains during late cellularization (Choi et al., 2013; Sawyer et al., 2009) and I was also able to confirm this finding for early cellularization.

GTPases are typically regulated by GEFs and GAPs, that can activate the GTPase by adding a GTP or inactivate it by addition of a GDP (reviewed in Cherfils and Zeghouf, 2013). To find the GEF that could activate Rap1 locally at the subapical domain, I first analyzed the known Rap1 GEFs in Drosophila. The first promising candidate was Dizzy, as it was found to play a role in Rap1 activation during ventral furrow formation in early gastrulation, acting upstream of E-Cadherin and adherens junctions (Spahn et al., 2012). Surprisingly, I could not detect a role for Dizzy in early subapical activation of Rap1, as dizzy mutant embryos did not show any Canoe mislocalization to the 
lateral domain, although Dizzy was found recently to mediate the localization of Canoe to tricellular junctions during the end of cellularization (Bonello et al., 2018). In addition, Bonello et al. (2018) could also not show a clear function of Dizzy on the lateral restriction of Canoe from the lateral domain during late cellularization. Furthermore, I could also show, that Bazooka and Armadillo, that are downstream of Canoe were not affected in dizzy mutants.

Another candidate for the activation of Rap1 was a complex formed by the proteins ELMO and Sponge, that were previously described as unconvential GEF for Rac and Rap1 in Drosophila and vertebrates (Biersmith et al., 2011; Yajnik et al., 2003). Maternal mutants for both genes missed actin caps during syncytial blastoderm stage and showed only partial invagination of cellularization furrows at the terminal poles of the embryo. As I could also show, that in invaginating furrows of these mutants subapical proteins like Canoe, Bazooka and Armadillo mislocalized to the lateral and sometimes even to the basal domain, it is likely that ELMO-Sponge could mediate the local activation of Rap1. Another strong argument for this interaction is the localization of both proteins, as I found Sponge to be subapically enriched in cellularizing wild type embryos and the GFP-tagged ELMO also showed subapical enrichment in living cellularizing embryos. In addition, both proteins showed a change in localization with onset of cellularization. During early syncytial blastoderm interphases both proteins localized in a disc-like pattern to the whole actin cap, whereas this localization changes to a more ring-like pattern with onset of cellularization and onset of zygotic gene expression (Figure 30A).

I hypothesize that these rings could give the spatial information for the insertion of the new subapical domain by definition of an interface of two already existing domains which becomes a new domain (Figure 30B). The change in localization of ELMO and Sponge could be mediated by mid blastula transition and the accompanied change from maternal to zygotic expression. This hypothesis is supported by the fact, that the subapical domain did not form in embryos with suppressed zygotic gene expression by the injection of $\alpha$-amanitin. Here I hypothesize, that a yet unknown gene could modify the localization upstream of ELMO-Sponge. Proteins that could mediate the localization of ELMO-Sponge are transport proteins like Dynein or Kinesin-1, that transport cargos along the cytoskeletal network. However, an interaction with transport proteins and ELMO was not shown yet, also not 
in other organisms. Though an interaction of the microtubule binding protein ACF7 (Short stop in Drosophila) with ELMO was shown in cell culture (Margaron et al., 2013). This interaction helps to capture and stabilize microtubules at the cortex to promote cell migration but does not give a hint for ELMO transport along the microtubules.

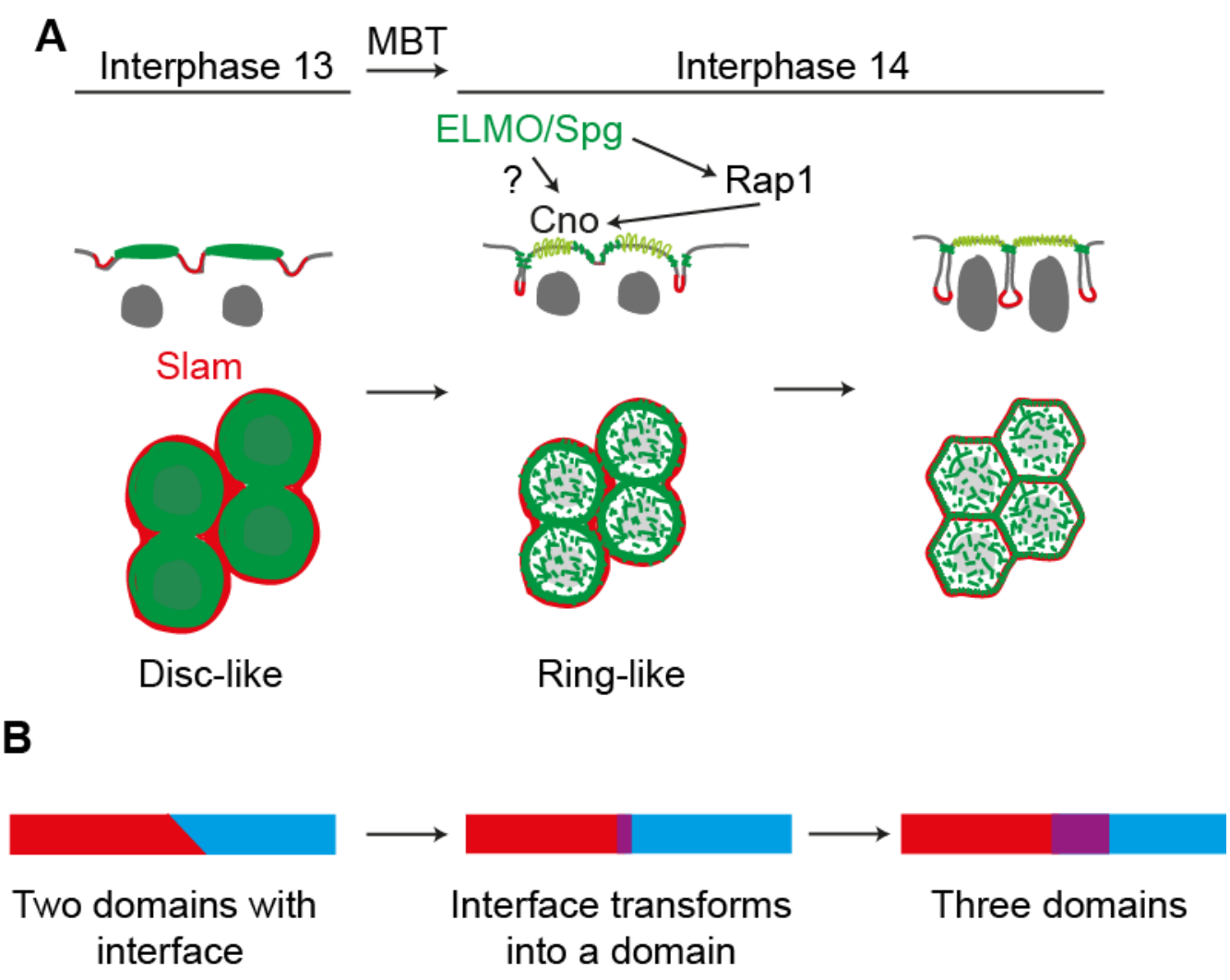

Figure 30 Model for the formation of the subapical domain during onset of cellularization.

(A) The ELMO-Sponge complex localizes in disc-like fashion to actin caps before MBT. Afterwards, with onset of cellularization, the complex localizes in a ring-like fashion and activates Rap1 locally. A further direct or indirect interaction with Canoe is not excluded. (B) Possible formation of a new domain by transformation of the interface.

Although, the pathway for the formation of the subapical domain is well described, the mechanistic relation between the proteins is not well understood yet. The protein Sponge itself is mainly acting together with the PH-domain protein ELMO (Biersmith et al., 2011; Yajnik et al., 2003). However, I could show, that ELMO is needed for the cortical localization of Sponge, as this is lost completely in ELMO mutants.

The ELMO-Sponge complex has been described to have various and partially redundant functions during developmental processes in different cell types of Drosophila. For example, Sponge shows cytoplasmic and membrane localization in air sac primordium cells of larval wing discs and cytoplasmic 
localization in R7 photoreceptor cells of larval eye discs (Eguchi et al., 2013; Morishita et al., 2017). In both tissues the knockdown of Sponge leads to developmental defects like apoptosis in air sac primordial cells and roughened eye phenotype by knock down in eye discs. The authors of the two studies could show, that in both tissues Sponge acts by activating the ERK pathway which is known to play a role in cell differentiation and apoptosis. However, whereas Sponge activates this pathway via Rac1 in the air sac primordium (Morishita et al., 2017), it acts via Rap1 on the differentiation of R7 photoreceptor cells (Eguchi et al., 2013). In these cell types Sponge does not act on cell polarization but on differentiation and cell maintenance. However, the activation of the ERK pathway downstream of ELMO-Sponge and Rap1 during cellularization is not likely, at least not for the formation of cortical domains, as a role for the ERK pathway for completion of cellularization is not described and the downstream cascade of Rap1 for positioning of subapical proteins is well researched. Although the mechanism of how Rap1 recruits Canoe, which recruits Bazooka are not known yet.

More research about the role of ELMO-Dock complexes during Drosophila development revealed also functions on the actin cytoskeleton and cell adhesion. During embryonal development the Sponge-related DOCK A protein Mbc together with ELMO activates Rac and functions via the actin cytoskeleton during dorsal closure (Toret et al., 2018) and myoblast fusion in muscle development (Biersmith et al., 2011). In contrast, the ELMO-Sponge complex acts in axon guidance during the embryonal development of the central nervous system where it is also thought to act on the actin cytoskeleton but here no experimental evidence is shown (Biersmith et al., 2011). Furthermore, $\mathrm{Mbc}$ and Sponge are both required for embryonal heart development. However, it seems that they exhibit different functions in this process, Mbc activating Rac which acts on the actin cytoskeleton, whereas Sponge acts via Rap1 possibly on cell adhesion (Biersmith et al., 2015).

In the mammalian system it was shown before, that the activation of Rap1 by DOCK4, the mammalian homologue of Sponge, could lead to cell adhesion by the formation of adherens junctions (Yajnik et al., 2003). In addition, Rap1 is necessary for the positioning and formation of adherens junctions in Drosophila wing disc epithelia (Knox and Brown, 2002). However, it was shown, that Rap1 is acting directly on E-Cadherin and Cadherin-binding proteins like Armadillo (Spahn et al., 2012). This direct interaction with E-cadherin for 
formation of cortical domains in my system is not likely, as subapical enrichment of E-Cadherin happens only during late cellularization, after the formation of cortical domains (reviewed in Laprise and Tepass, 2011). Furthermore, it was shown before, that Rap1 acts on Canoe during late cellularization probably by direct binding, as Canoe has a Rap1 binding site (Boettner et al., 2000; Boettner et al., 2003; Linnemann et al., 1999). I could also show, that the function of Rap1 is necessary for Canoe localization during early cellularization, which supports the hypothesis, that Rap1 acts via Canoe on the formation of the subapical domain.

However, although Canoe shows a massive mislocalization in Rap1 mutants with no cortical enrichment at all, Canoe still shows cortical localization in sponge and ELMO mutants with subapical enrichment but enhanced signal along the lateral domain. There are several possibilities that could explain this contrast. Firstly, it is possible, that also other Rap1 GEFs contribute to the correct spatial activation of Rap1. Although I could show that the known Rap1 GEF Dizzy did not contribute to Canoe localization, there are several more known Rap1 GEFs in Drosophila which are not investigated during cellularization yet. Secondly, another yet unknown downstream effector of Rap1 could mediate the spatial restriction of Canoe. This hypothesis is also likely as subapical Canoe localization is much more prominent and restricted than Sponge localization. Furthermore, several Rap1 effectors are known, which directly or indirectly regulate the cytoskeleton and also other Rap1 GEFs like Epac (reviewed in Bos, 2006).

The subapical marker Bazooka, which is known to bind E-cadherin and can recruit it to adherens junctions, gets localized during the course of cellularization. Although it was described before, that it also localizes to the lateral domain of metaphase furrows (Harris and Peifer, 2004), I was not able to detect it during mitosis in fixed or living embryos. In addition, Bazooka did not mark the subapical domain during onset of cellularization but got enriched there later. In living embryos, I was only able to detect Bazooka puncta during mid-cellularization. As described before, the subapical protein enrichment is mediated by Dynein transport along the microtubule network and needs a cortical actin scaffold (Harris and Peifer, 2004). How Canoe does interact with Bazooka is not clear yet. Choi et al. (2013) could show, that Canoe was able to recruit Bazooka in vitro although both proteins do not necessarily colocalize, as well in the cell culture system as also during cellularization. As Canoe and 
Bazooka both are PDZ-domain proteins, a direct interaction via these domains could be possible. Another possibility is that Rap1/ Canoe modify the cytoskeleton in a way, that Bazooka could find its subapical position during the course of cellularization, as it was shown before, that Canoe could do so during apical constriction (Sawyer et al., 2009). However, Choi et al. (2003) could show, that the cytoskeleton is not modified in canoe and Rap1 mutant embryos, making this hypothesis unlikely.

Taken together, with my work I could show, that the unconventional GEF complex ELMO-Sponge is upstream of Rap1, activating it locally at the subapical domain, leading to subapical localization of Canoe with onset of cellularization. ELMO-Sponge itself could be found at the interphase caps during syncytial blastoderm development in a disc-like pattern, whereas with switch from maternal to zygotic gene expression they change their localization to a more ring-like pattern, possibly defining the place of the newly introduced subapical domain. 


\section{Part B}

Role of Dia for the formation of cortical domains and organization of cortical actin 


\section{Abstract}

The localization of the subapical proteins Canoe and Bazooka does not only depend on its upstream factors Canoe and Rap1 but also on a functional cortical F-actin scaffold. The depolymerization of F-actin by injection of drugs leads to perturbed localization of Canoe and Bazooka. Embryos mutant for the F-actin nucleator and elongator diaphanous (dia) do not only display markers of the lateral domain like Dlg leaking into the basal cortical domain but I could also show mislocalization of Canoe, Bazooka and Armadillo. Although, this observation led to the hypothesis, that the mislocalization of subapical proteins in dia mutants happens due to less or perturbed cortical F-actin, I could not find differences in F-actin amount of dia versus wild type embryos during syncytial blastoderm development. Also, during cellularization a mislocalzation of F-actin was not detectable. Dia was described to localize to the barbed ends of the actin filaments to elongate the filaments at this position. By staining of syncytial blastoderm embryos, I could show that the interphase actin caps show some polarization with Dia-marked barbed ends being enriched at the cap edges. This observation led to the hypothesis, that Dia could be responsible for the organization and polarity of the cortical F-actin network in actin caps. I was able to confirm this hypothesis by staining with the barbed-end marker Capping protein alpha (Cpa) in wild type and dia syncytial blastoderm embryos. Cpa showed significant less enrichment at the edges actin caps in dia embryos. I hypothesize, that the polarized cortical Factin network acts as a prepattern that defines the position of the subapical domain with onset of cellularization and could direct the ELMO-Sponge complex for formation of the subapical domain to its ring-like localization. Although the polarization of the cortical F-actin is available before cellularization I hypothesize that it is read out with midblastula transition and onset of zygotic gene expression. In this hypothesis Dia plays a more indirect role for the formation of the subapical domain, going in line with the observation that Dia does not show a subapical enrichment during early cellularization as well as I could not detect elevated subapical Rho signaling that could explain local subapical Dia activation. 


\section{Introduction}

\subsection{Actin dynamics are controlled by actin binding proteins}

Actin binding proteins, that can nucleate, elongate or bundle actin filaments are a group of conserved proteins among the animal kingdom (reviewed in Pollard et al., 2000). In Figure 31 the dynamics of non-muscle actin are depicted. Profilin-bound globular actin monomers can be nucleated by a formin to build an actin filament which is also elongated by this protein class at their barbed (or plus) ends (Kovar et al., 2006; Pring et al., 2003). At pointed (or minus) ends of filaments, depolymerization happens spontaneously. The elongation of a filament can be stopped by binding of capping proteins to the plus ends, removing the formin from its place (reviewed in Pollard et al., 2000).

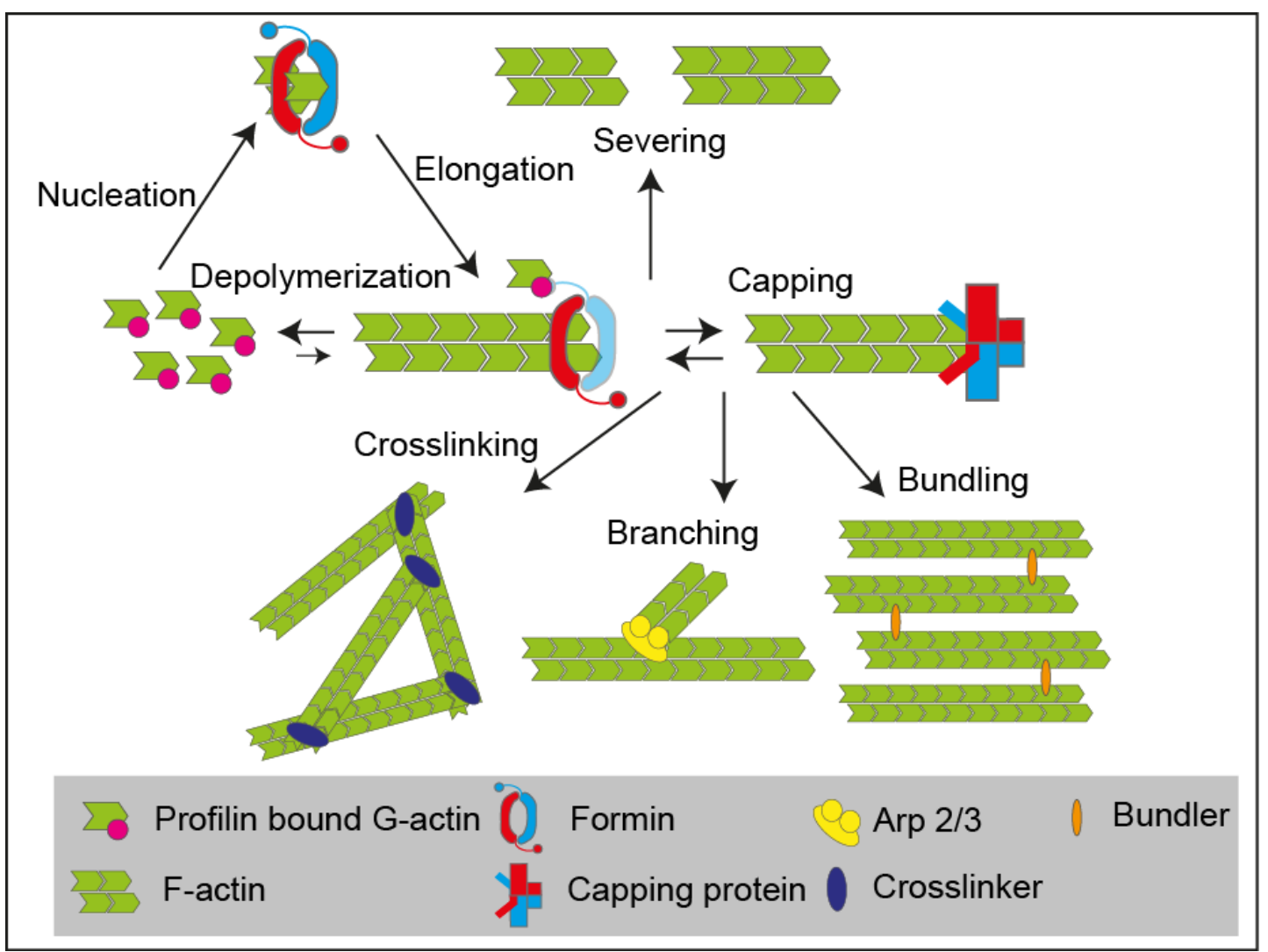

Figure 31 Scheme of actin dynamics in non-muscle cells.

Actin filaments are nucleated from monomeric actin molecules and elongated to form long filaments. These filaments can be severed, crosslinked, bundled or branching can occur.

However, recent publications point out, that both, Capping proteins and formins, can bind to barbed ends simultaneously at least for short terms (Bombardier et al., 2015; Shekhar et al., 2015). Next to severing of filaments, they can also be bundled to thick filaments or being crosslinked (Figure 31). The formation of branches, which emerge in an angle of $70^{\circ}$ is nucleated by 
the Arp 2/3 complex (Mullins et al., 1998) (Figure 31). The formation and dynamics of the actin cytoskeleton are not only crucial for cell maintenance but also for cell mobility, cytokinesis and also for cell-cell interaction (reviewed in Bogdan et al., 2013; Pollard et al., 2000).

\subsection{The formin Dia nucleates and elongates filamentous actin and binds to plus ends}

The protein Dia belongs to the protein family of formins, which has a sole homolog in Drosophila (reviewed in Bogdan et al., 2013). Typical of proteins belonging to the Dia-family are their domain composition and regulation (Figure 32; reviewed in Bogdan et al., 2013).

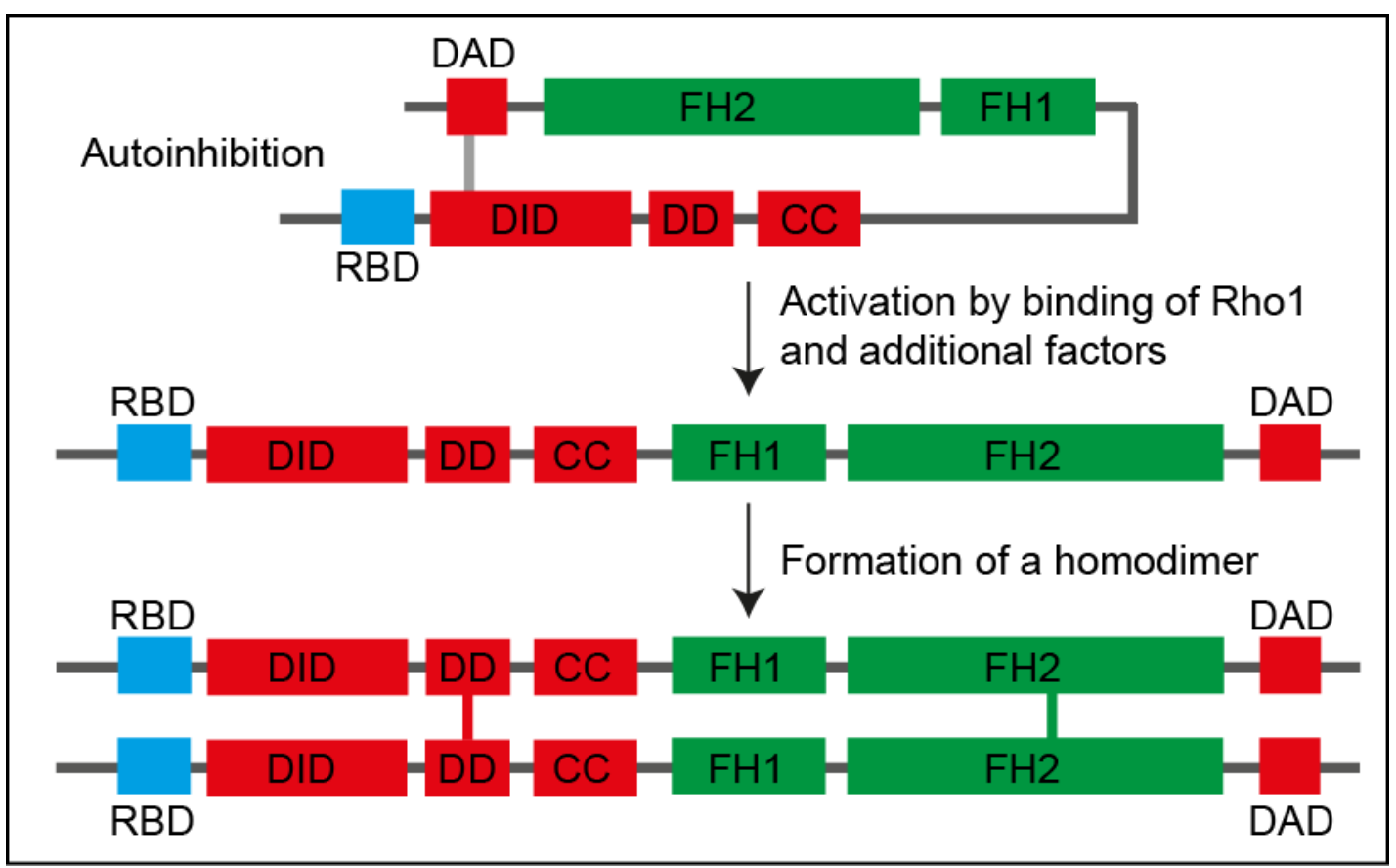

Figure 32 Scheme of domain composition of Drosophila Dia.

In the autoinhibited state, the DAD domain binds to the DID domain. This interaction is released upon binding of Rho1 to the RBD domain, followed by the formation of a homodimer to nucleate and elongate F-actin. Abbreviations: Rho binding domain (RBD), Dia inhibitory domain (DID), dimerization domain (DD), coiled-coil domain (CC), Formin homology domain 1 and 2 (FH1, FH2), Dia autoinhibitory domain (DAD) (Bogdan et al., 2013; Kühn and Geyer, 2014).

Drosophila Dia is composed by an N-terminal Rho-binding domain to which Rho1 binds for activation. Followed by 3 domains being necessary for regulation, the Dia inhibitory domain (DID), the dimerization domain (DD) and a coiled-coil region. In autoinhibited state, the C-terminal Dia autoregulatory domain (DAD) binds to the DID domain. This binding is released by binding of Rho1. After activation and formation of a Dia 
homodimer, the Formin homology domains 1 and 2 (FH1, FH2) are responsible for nucleation and elongation of F-actin. In this process, the FH2 domains form a ring and bind to the growing barbed end of the actin filament whereas the FH1 domain recruits profilin-bound G-actin to the growing filament (reviewed in Bogdan et al., 2013; Kühn and Geyer, 2014). Dia can act in three ways on actin. The first activity is the elongation function in which Dia binds to the actin filament and includes profilin bound G-actin to the tip of the growing filament (Großhans et al., 2005; Yan et al., 2013). The second activity is the nucleation of three profilin-bound G-actin units to form a new actin filament (Li and Higgs, 2003). The third activity is its binding to the plus ends of the actin filament, being able to remove capping proteins (reviewed in Pollard et al., 2000). However, recent publications show evidence, that at least in vitro the FH2 and FH1 domain of formins can bind in parallel to capping proteins which leads to a so-called decision complex controlling the dynamics of the filament (Bombardier et al., 2015; Shekhar et al., 2015).

\subsection{Multiple functions of Dia in Drosophila development}

The diverse functions of Dia during Drosophila development are well researched (reviewed in Bogdan et al., 2013). It functions during cytokinesis by formation of the contractile ring for example in spermatogenesis in male testis (Castrillon and Wasserman, 1994). Furthermore, Dia seems to play a role in cell shape changes by acting upstream of actomyosin contraction and stability at adherens junctions during Drosophila morphogenesis (Homem and Peifer, 2008). During cellularization, which is also described as special form of cytokinesis, Dia is necessary for proper membrane invagination and for the stabilization of the basal furrow canal, as described before in chapter 2.6 (Afshar et al., 2000; Großhans et al., 2005; Yan et al., 2013). Furthermore, Dia plays a role in planar cell polarity during the formation of wing hairs by acting on cytoskeletal activation and directly on the planar cell polarity pathway ( $\mathrm{Lu}$ and Adler, 2015).

\subsection{Aim of the thesis}

In this part of my thesis, I analyzed the function of the formin Dia in the formation of the subapical domain. I was able to show, that Dia plays a role for the introduction of the subapical domain and for the localization of subapical proteins. Furthermore, I could show, that Dia acts by the organization and polarization of the cortical F-actin network of the syncytial 
blastomderm embryo. This polarization of the cortical F-actin could serve as a pattern which is red out during onset of cellularization and gives rise to the subapical domain. Here I was able to connect the permissive, indirect function of Dia and the cortical F-actin with the localization of subapical proteins. 


\section{Results}

\section{1 dia and bazooka show antagonist genetic interaction}

\section{Dia protein is strongly reduced in dia mutant embryos}

To investigate the function of F-actin during cortical domain formation, I made use of a dia mutation. The allele dia $a^{\text {sy5 }}$, that was used for this study, from here on named as dia, carries a point mutation leading to the exchange of the Serin at position 92 by a Leucin, resulting in a hypomorphic mutant situation (Yan et al., 2013) (Figure 33).

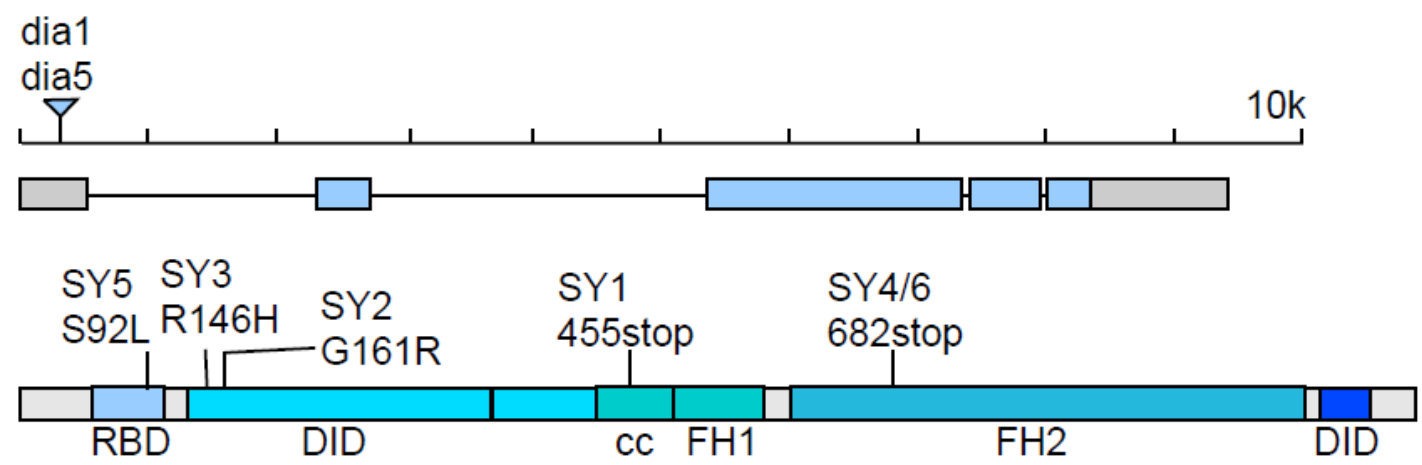

Figure 33 Genetic region of dia and alleles.

Coding sequence of dia locus is indicated by blue boxes, 5'UTR and 3'UTR by grey boxes. dia1 shows an inserted p-element (Castrillon and Wasserman, 1994) that was mobilized to generate dia5 (Afshar et al., 2000). Lower boxes indicate Dia protein. Point mutations characterized by sequencing with changes in amino acid sequences are indicated (SY1-6). Figure was taken from Yan et al. (2013).

As the effect of the mutation on the protein level is not understood, I stained wild type and dia embryos with an antibody raised against the C-terminal half of Dia that mediates the function on F-actin (Figure 34A, B). To ensure comparable experimental conditions, dia embryos that expressed a GFPtagged version of MyoII were used and stained in the same tube together with unmarked wild type embryos. Whereas Dia was enriched at the basal tip of cellularization furrows at early cellularization in wild type embryos it was not detectable in dia germ line clones (Figure 34A). The lateral domain was stained by Dlg, which was also detectable at the furrow canal in dia mutants (Figure 34A), a phenotype described before by Yan et al. (2013). To analyze total Dia amounts in wild type versus dia embryos, I used extracts from embryos which were incubated for 2 hours after egg laying at $25^{\circ} \mathrm{C}$ and made a western blot and stained against Dia (Figure 34B). The protein could be detected at $130 \mathrm{kDa}$ 
in wild type whereas only a faint band was detectable in dia embryonic extracts. As loading control, $\alpha$-Tubulin was used.
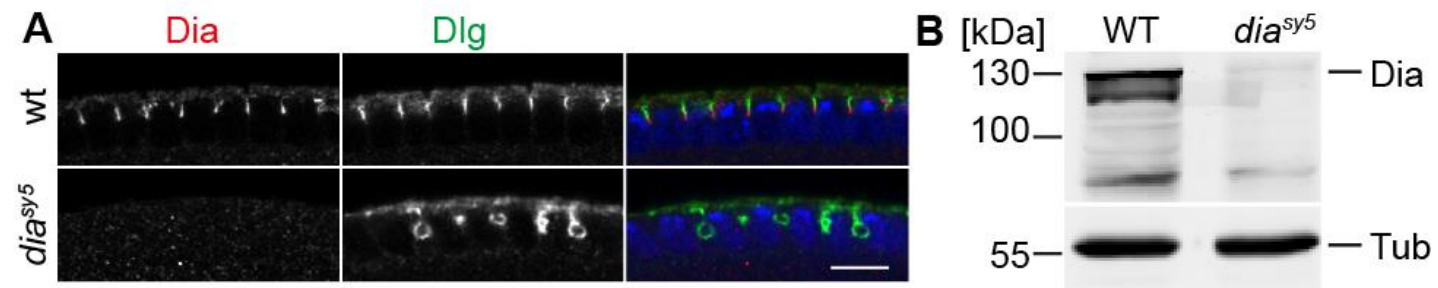

Figure 34 Dia is reduced in dia mutants.

(A) Fixed wild type and dia embryos during early cellularization stained against Dia (grey/ red), Dlg (grey/green) and DNA (blue). Embryos of both phenotypes were co-stained and imaged with same laser settings. (B) Western blot of extracts from wild type and dia glcs 0-2 $\mathrm{h}$ after egg laying against Dia. $\alpha$-Tubulin was used as loading control. 15 embryos per lane were loaded. Scale bar $10 \mu \mathrm{m}$.

\section{Overexpression of Bazooka in dia mutant background leads to defects during oogenesis}

To analyze the dependence of Dia on Bazooka, I wanted to describe Bazooka dynamics in wild type and dia mutant background. However, crossing of a GFP-tagged UASp-Bazooka driven by a maternal promoter into dia mutant background resulted in flies that did not lay eggs. In contrast, the expression of BazookaGFP at endogenous levels (Protein Trap) in dia mutant background, gave rise to viable embryos which showed the dia phenotype. In this fly line, a GFP is inserted by cloning of an artificial exon, which encodes GFP, into the genomic locus, resulting in a GFP-tagged protein expressed in endogenous amounts from its own promotor (2x Bazooka) (Buszczak et al., 2007; Morin et al., 2001). To investigate this antagonistic genetic interaction, I generated dia germline clones overexpressing BazookaGFP (dia $+3 x$ Bazooka). Mutant germline cells were marked by absence of nuclear GFP. I dissected the ovaries of these flies and stained against Phalloidin and DNA to show the structures of the ovary (Figure 35). In wild type stage 9 ovaries, expressing 2xnlsGFP which marked the nuclei with GFP, the boundary between nurse cells and oocyte was stained by a strong Phalloidin signal, which stains F-actin (Figure 35, red arrow). In dia mutant clones which were overexpressing BazookaGFP (dia $+3 x$ Bazooka), marked by absence of GFP from nuclei of germ cells, this Phalloidin staining at the border between oocyte and nurse cells was diminished. Instead the oocyte seemed to be filled with F-actin meshwork (Figure 35B, red arrow). Furthermore, in dia mutant ovaries with BazookaGFP overexpression the nuclear integrity seemed to be lost, as heterochromatin, 
marked by stronger DAPI staining, seemed to be disorganized. Stage 9 ovaries were the oldest stages which could be detected in this genetic background. To conclude these findings, bazooka and dia show an antagonistic genetic interaction, as the overexpression of bazooka in dia mutant background led to phenotypes during oogenesis which resulted in degradation of the developing egg chambers. Overexpression of BazookaGFP in wild type background (3x Bazooka) or expression of BazookaGFP in endogenous amounts in dia background (dia $+2 x$ Bazooka) did not give rise to the described phenotypes and resulted in eggs which could further develop. To make sure, that the oogenesis was not affected by the GFP-tag of overexpressed Bazooka in dia background, I made use of an mCherry-tagged Bazooka, which was driven by a ubiquitin-promoter. However, also the overexpression of CherryBazooka in dia mutant background led to perturbed oogenesis.

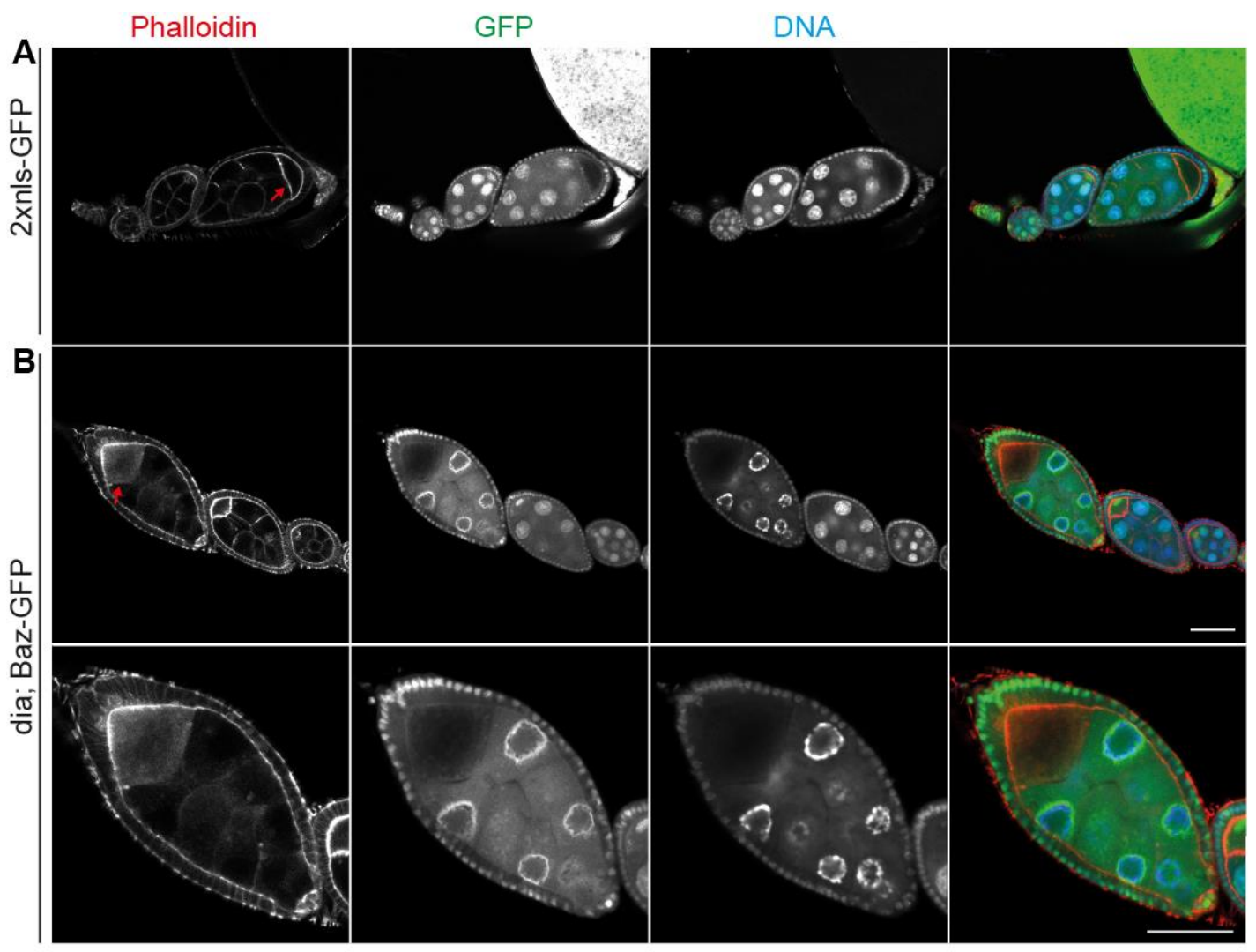

Figure 35 Antagonistic genetic interaction of dia and baz during oogenesis.

Ovaries from (A) flies expressing 2xnls-GFP and (B) flies with dia mutant germline, marked by no nuclear GFP staining, overexpressing BazGFP. Ovaries are stained for F-actin by phalloidin (grey/red), DNA (grey/blue) and GFP (grey/green). The red arrows mark the border between nurse cells and oocyte. Lowest panel shows zooms from middle panel. Scale bar $50 \mu \mathrm{m}$. 


\subsection{Dia is required for subapical localization of marker proteins}

As described in chapter 3.2, Canoe localization is dependent on the GTPase Rap1 which is locally activated by the unconventional GEF-complex ELMOSponge. Downstream of Canoe, the PDZ-domain protein Bazooka gets localized during the course of cellularization (Choi et al., 2013). Bazooka binds E-cadherin and directs this protein to the subapical domain to set up adherens junctions after cellularization (Harris and Peifer, 2004). The localization of Bazooka does not only depend on Canoe and Rap1 but also on a cortical Factin scaffold, to hold it at its place (Harris and Peifer, 2005). To get further insight into the formation of the subapical domain, I tested the localization of Bazooka and its downstream target Armadillo in wild type and different genetic backgrounds (Figure 36).

\section{The localization of Armadillo and Bazooka depends on Dia and Rap1 but not on Dizzy}

In dia mutant embryos, Bazooka mislocalized even to the basal domain during early cellularization and spread over the whole membrane and was still found at the lateral domain during mid-cellularization (Figure 36B). Armadillo localized to the lateral domain during cellularization and was enriched in spot junctions at the subapical domain and the basal end of the lateral domain in wild type embryos (Figure 36A) (Harris and Peifer, 2004; McGill et al., 2009). In dia mutants, Armadillo lost its subapical enrichment and also spread into the basal domain (Figure 36B). As described before (Choi et al., 2013) not only Canoe localization but with this also Bazooka localization depends on the GTPase Rap1. I repeated this experiment and indeed Bazooka puncta were spread over the whole lateral domain during mid-cellularization and also in late cellularization, Bazooka puncta were still visible at the lateral domain (Figure 36C). Furthermore, the subapical enrichment of Armadillo was lost, although the enrichment at basal junctions was still visible, as shown before by Choi et al. (2013) (Figure 36C). Dizzy was described as potential Rap1GEF during cellularization to locally activate Rap1 (Choi et al., 2013; Spahn et al., 2012). However, as I could already show for Canoe, Bazooka and Armadillo localization was not perturbed in dizzy mutant embryos in early and late cellularization (Figure 36C) compared to wild type (Figure 36A). 


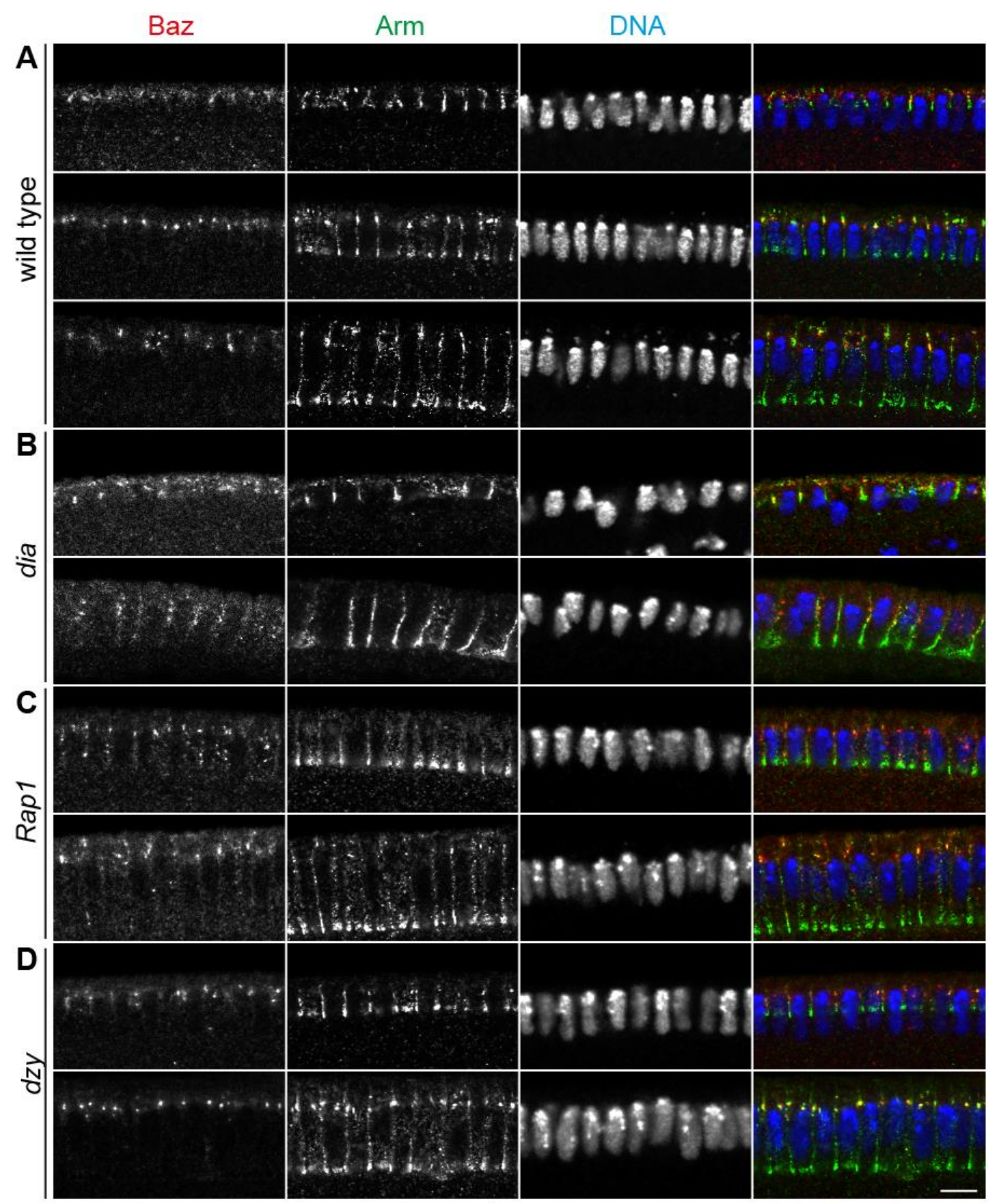

Figure 36 Genetic control of subapical Baz and Arm localization.

Images of fixed embryos in early, mid and late cellularization stained for Baz (grey/red), Arm (grey/green) and DNA (grey/blue). Merged images are shown in right panel. Genotypes (A) wild type, (B) dia, (C) Rap1 and (D) dzy as indicated. Scale bar $10 \mu \mathrm{m}$.

\section{Dia is required for subapical localization of Armadillo}

To get further insight into the perturbed localization of Armadillo in dia mutant embryos, I measured the fluorescence intensity of the Armadillo signal along three furrows of three embryos in apical-basal direction in wild type and dia (Figure 37A, B). I normalized the data to its peak and plotted relative 
fluorescence intensities along the 9 furrows as heatmaps and averaged for wild type and dia (Figure 37C).

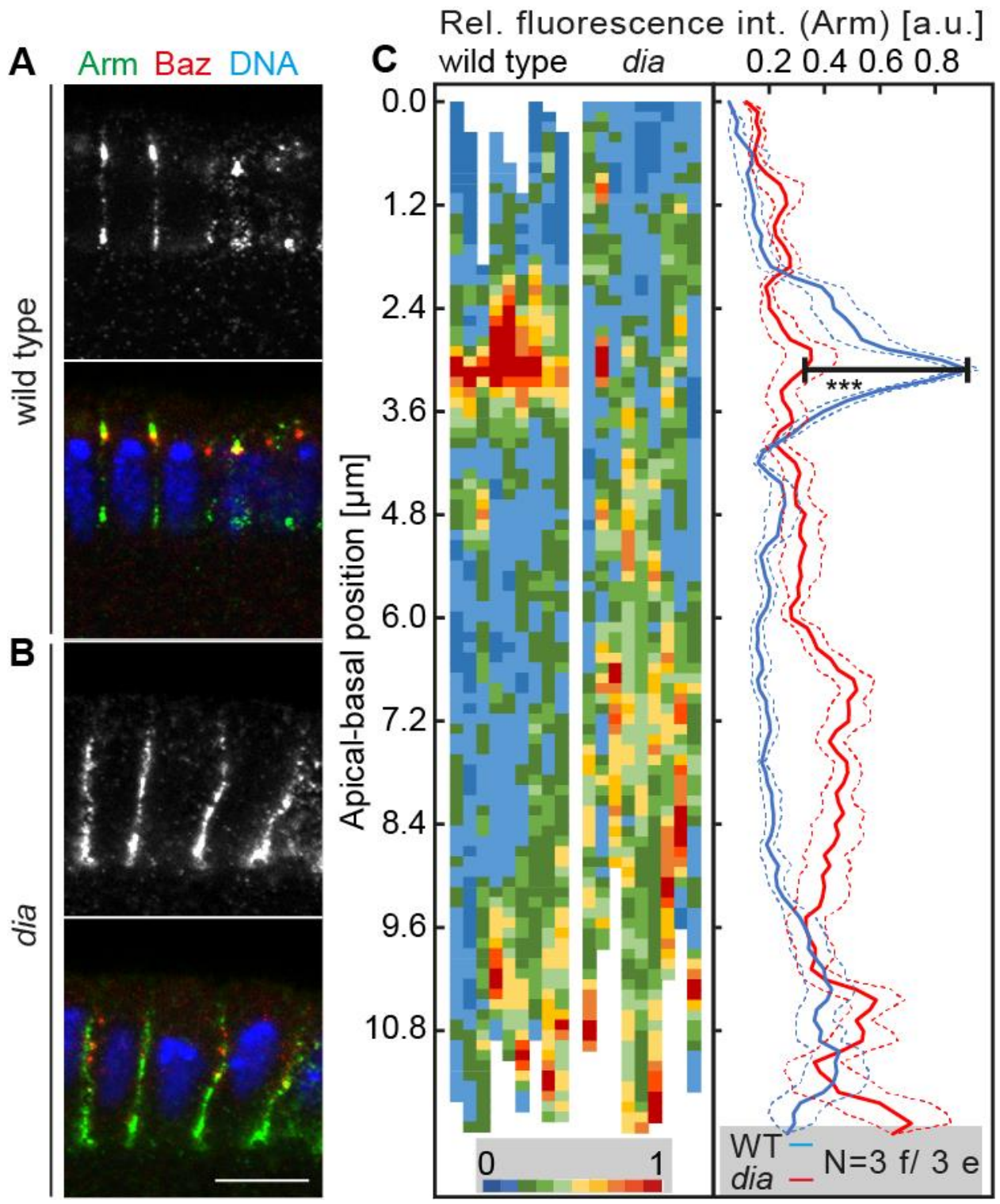

Figure 37 Dia is required for subapical Arm enrichment.

(A-B) Images of fixed (A) wild type and (B) dia mutant embryos stained for Arm (grey/green) Baz (red) and DNA (blue), merged images are shown in lower panels. (C) Relative fluorescence intensity of Arm signal measured along the apical-basal axis of 9 furrows in 3 wild type and dia embryos. Heatmaps (left) display relative fluorescence intensities of each measured furrow. Averages were plotted on the right side. The bar represents the difference of relative fluorescence intensities at the peak $(p<0.001)$. Dashed lines indicate SEM. Scale bar $10 \mu \mathrm{m}$.

The apical-basal position of intensities measured in wild type embryos were aligned to the individual peaks, which was not possible in the mutant situation due to the dispersed signal. The heatmaps showed a clear subapical enrichment and also some basal enrichment in wild type embryos as described before (Harris and Peifer, 2004; McGill et al., 2009). In dia mutants, the 
subapical enrichment of the Armadillo signal was clearly diminished and relative Armadillo signal was higher along the lateral domain. The averages of the Armadillo signal showed a clear subapical peak in wild type compared to no clear peak in dia embryos (Figure 37C). This difference at the subapical domain was highly significant with a p-value $<0.001$.

\section{Subapical Bazooka enrichment depends on Dia}

As Armadillo is downstream of Bazooka, I wanted to analyze if the mislocalization of Armadillo was due to mislocalized Bazooka in dia mutants. For this, I also stained wild type and dia mutant embryos for Bazooka and quantified fluorescence intensities along the cellularization furrows as explained before. Figure 38A, B shows fixed wild type and dia mutant embryos stained for Bazooka and Armadillo as already shown in Figure 37A, B. In wild type embryos Bazooka puncta accumulated at the subapical region, showing some overlap with Armadillo, whereas Bazooka puncta were distributed along the lateral domain in dia mutant embryos. I quantified this observation as shown before (Figure 38C). In wild type embryos, the subapical enrichment of Bazooka signal was visible as well as the lateral restriction of the Bazooka signal, whereas subapical Bazooka peaks were not visible in dia mutants. Instead elevated relative Bazooka signal was visible along the whole furrow. Apparently, by averaging the intensities of the Bazooka signal in all furrows, Bazooka signal was elevated along the lateral domain in dia mutants compared to wild type leading to the difference in the base lines (Figure 38C). The subapical peak was clearly visible in wild type embryos and showed a significant difference to the signal in dia embryos with a p-value $<0.001$. 


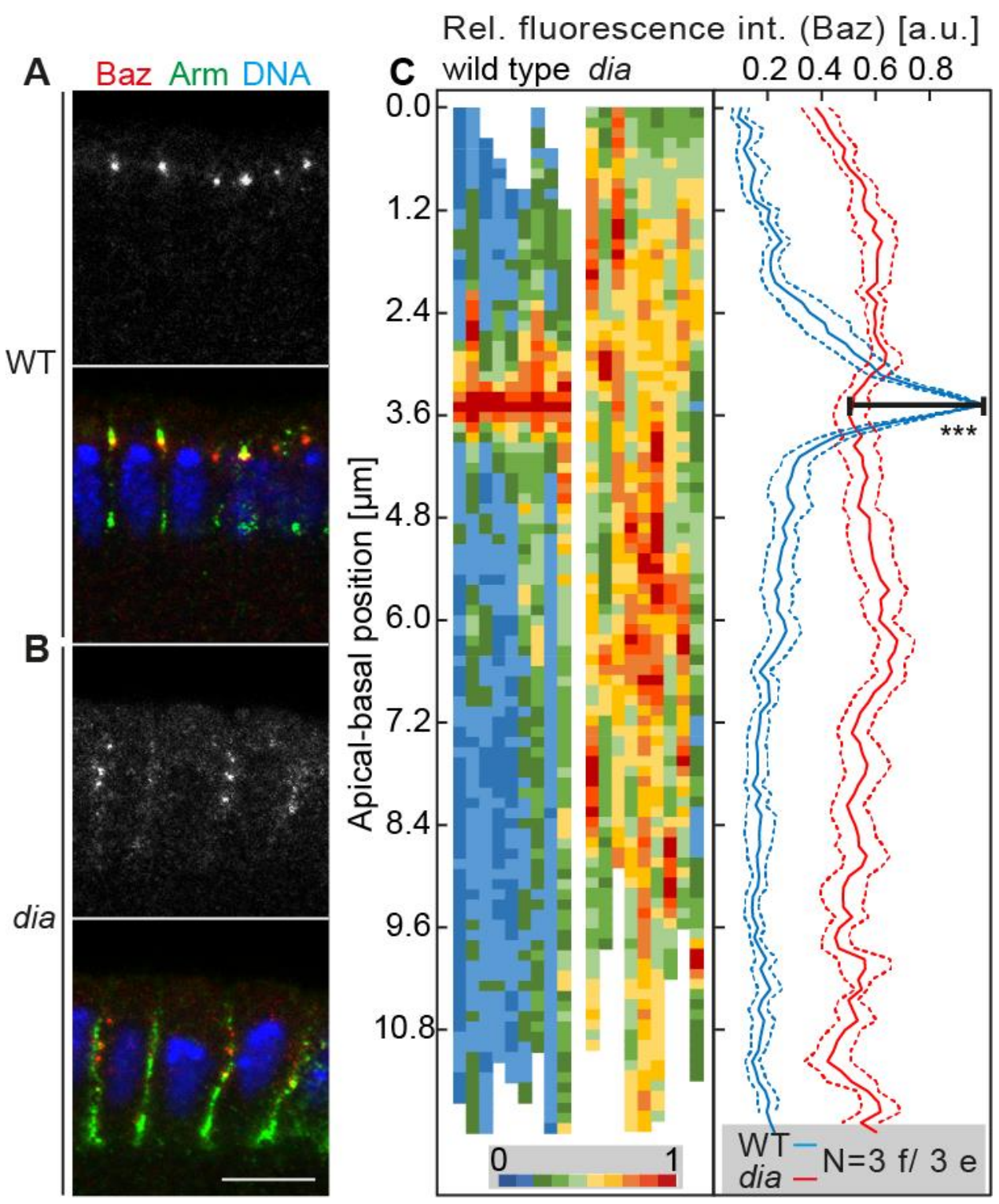

Figure 38 Dia is required for subapical Baz enrichment.

(A-B) Images of fixed (A) wild type and (B) dia mutant embryos stained for Baz (grey/red), Arm (green) and DNA (blue). Merged images are shown in lower panels. (C) Heat maps (left) of relative fluorescence intensities of Baz signal measured along the apical-basal axis of 9 furrows in 3 wild type and dia embryos. Averages (right) of relative fluorescence intensities plotted against apical-basal position. Bar represents difference of both genotypes at the peak. $\mathrm{P}<0.0001$. Dashed lines indicate SEM. Scale bar $10 \mu \mathrm{m}$.

\section{Subapical enrichment of Bazooka is delayed in dia embryos}

Next to the stainings against Bazooka in wild type and dia mutant embryos, I wanted to analyze the dynamics of Bazooka during cellularization in living embryos. For this purpose, I imaged wild type and dia mutant embryos expressing BazookaGFP in endogenous levels as already shown in Figure 24 with a two-photon microscope in intervals of $10 \mathrm{~min}$. (Figure 39). Timepoint 0 was defined by the appearance of interphase 14 nuclei. In wild type embryos expressing BazookaGFP, subapical Bazooka signal got visible after around 40 
min, a timepoint that should reflect mid cellularization (Figure 39A). However, as I did not introduce a membrane marker, the progress of membrane invagination could not be followed. In dia mutant embryos (Figure 39B) the subapical BazookaGFP signal was only visible at a time point of around 50-60 min, that should reflect the end of cellularization. As the nuclei were not arranged in one horizontal plane at $60 \mathrm{~min}$, gastrulation might have started at this time point. It is possible, that the delayed subapical BazookaGFP signal was due to the more dispersed localization of Bazooka in dia mutant embryos. However, it is also possible, that this is due to less BazookaGFP, as it was expressed homozygously in wild type and only heterozygously in dia mutant background.

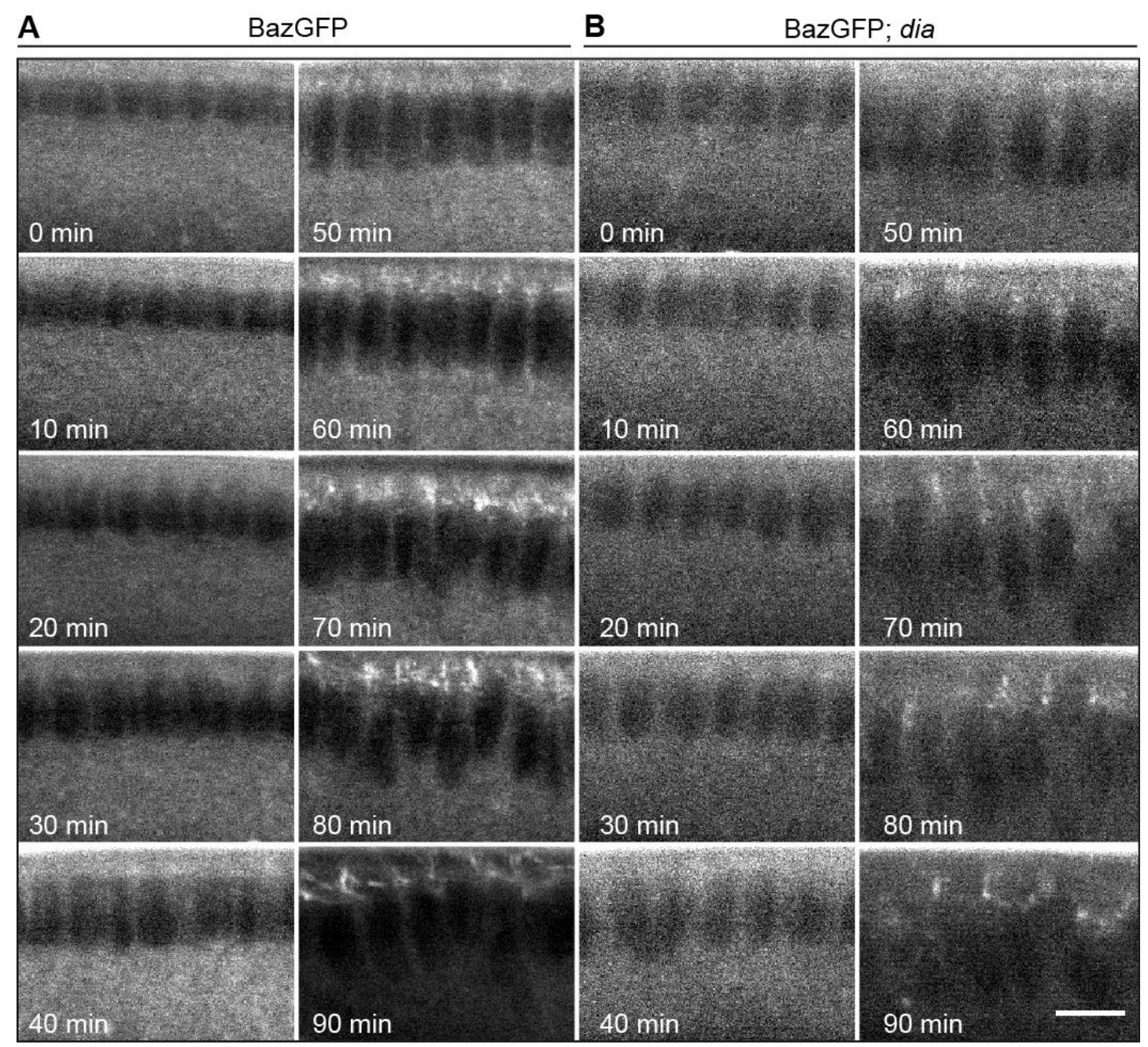

Figure 39 Dynamics of Baz-GFP during cellularization in wild type and dia embryos.

(A-B) Images from a movie of (A) WT and (B) dia embryos expressing BazGFP during cellularization. Movies were taken with a 2-photon microscope to enable sagittal views. Scale bar $10 \mu \mathrm{m}$. 


\section{Canoe shows subapical localization in dia mutants but is dispersed along the lateral membrane}

The investigation of the localization of Bazooka and Armadillo during early cellularization is difficult because in stainings of fixed embryos, subapical signal is not reliably visible during this time points. To get further insight into subapical localization of marker proteins in dia mutants, I made use of the marker protein Canoe which shows reliable subapical localization already during early cellularization, as I could show before. Furthermore, Canoe is needed for subapical localization of Bazooka and its mislocalization in dia could be explained if also the restriction of Canoe from the lateral domain is defective. To examine Canoe in dia mutants, I stained fixed wild type and dia mutant embryos and quantified the fluorescence intensity of the Canoe signal as before for Armadillo and Bazooka (Figure 40).

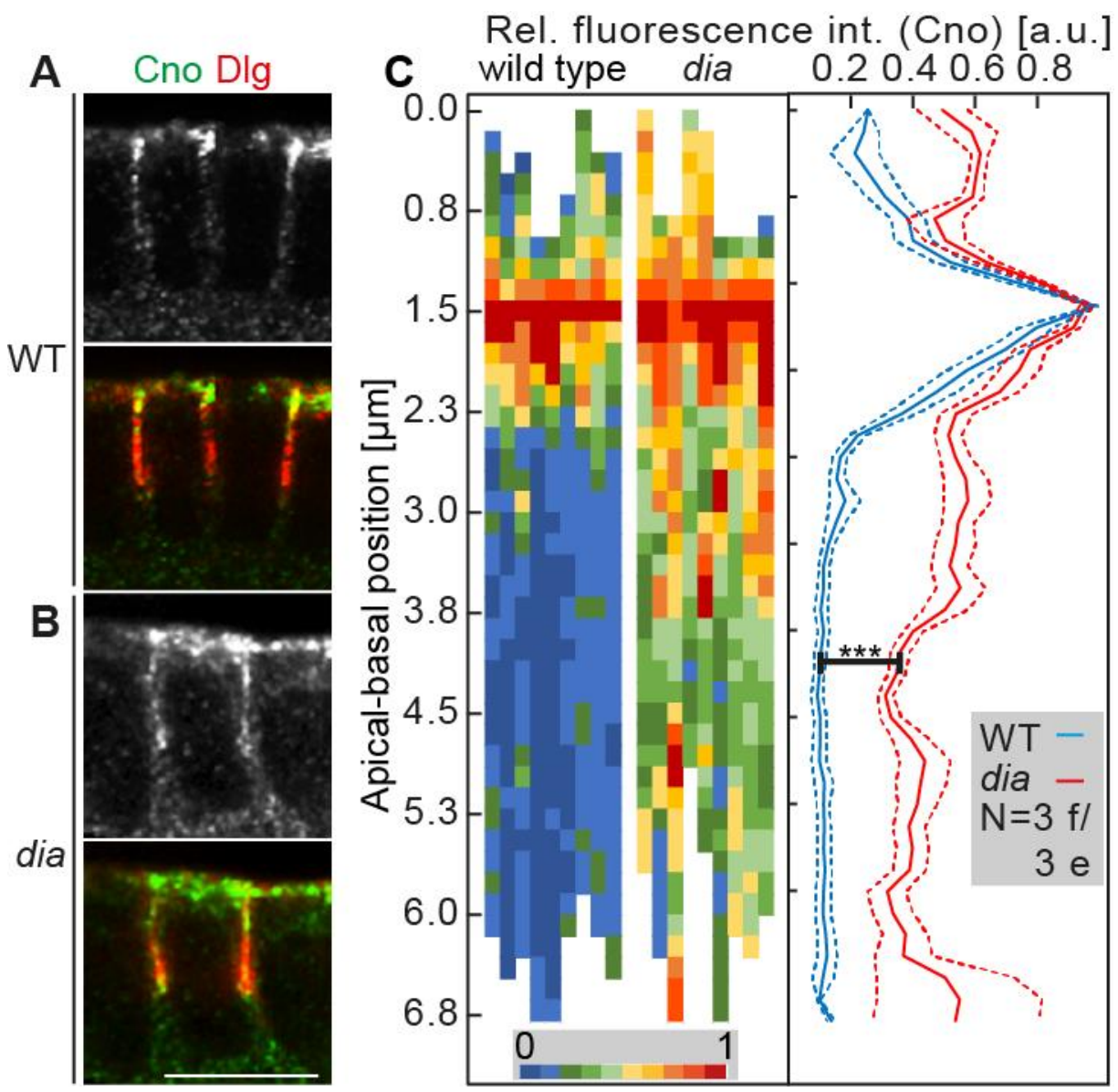

Figure 40 Dia is required for subapical Cno enrichment.

(A-B) Images of fixed (A) wild type and (B) dia mutant embryos stained for Cno (grey/green) and Dlg (red). Merged images are shown in lower panels. (C) Heat maps (left) of relative fluorescence intensities of Cno signal measured along the apical-basal axis of 9 furrows in 3 wild type and dia embryos. Averages are shown on right side. The difference of signal intensities is shown with a black bar. $\mathrm{P}<0.0001$. Dashed lines indicate SEM. Scale bar $10 \mu \mathrm{m}$. 
Figure 40A, B shows wild type and dia embryos during early cellularization stained for Canoe and Dlg. In wild type embryos Canoe localized to the subapical domain and was restricted from apical and lateral domains whereas in dia Canoe is also visible at the lateral and also at the apical domain. To quantify if Canoe is not properly restricted from the lateral domain in dia mutants, I measured the fluorescence intensities along three furrows in three wild type and dia embryos as shown before (Figure 40B). In wild type embryos a clear subapical localization of Canoe was visible and also relatively weak signal along the lateral domain, whereas Canoe signal seemed not restricted from the lateral domain in furrows from dia embryos, as a relatively high Canoe signal was visible also at lateral positions. However, compared to the localization of Bazooka and Armadillo in dia, Canoe still showed a subapical peak. This made it possible to also normalize the apical-basal position from the measurements in dia to their peaks (Figure 40C). Also, the averages plotted in Figure 40C make clear. that a subapical peak could be detected in wild type as well as in dia embryos. However, the restriction of Canoe from the lateral domain was perturbed in dia, as the difference between the two relative fluorescence intensities of both genotypes was highly significant as represented by the bar in Figure 40C (p-value $<0.001$ ).

\section{The localization of ELMO-Sponge depends on Dia}

The ELMO-Sponge complex showed subapical enrichment and was required for the local activation of Rap1 and subsequently localization of Canoe, Bazooka and Armadillo, as I could show before in part A of this thesis. The fact, that the proteins Canoe and Bazooka were mislocalized in dia mutant embryos lead to the analysis of their upstream factor Sponge in dia mutants. As performed for the other proteins, I stained fixed cellularizing wild type and dia embryos against Sponge and Dlg (Figure 41A, B). In wild type, Sponge signal was detectable along the whole cellularization furrow, but showed a clear enrichment at the subapical region (Figure 41A). Also, in dia mutants Sponge was detectable along the cellularizing furrow and also showed subapical enrichment although the staining gave the impression, that the subapical enrichment was not as clear as in wildtype and Sponge also seemed to spread into the apical domain (Figure 41B). To quantify the localization of Sponge, I measured the fluorescence intensity of the Sponge signal in 19 furrows of four wild type and dia mutant embryos along the apical- basal axis as done before (Figure 41C). 


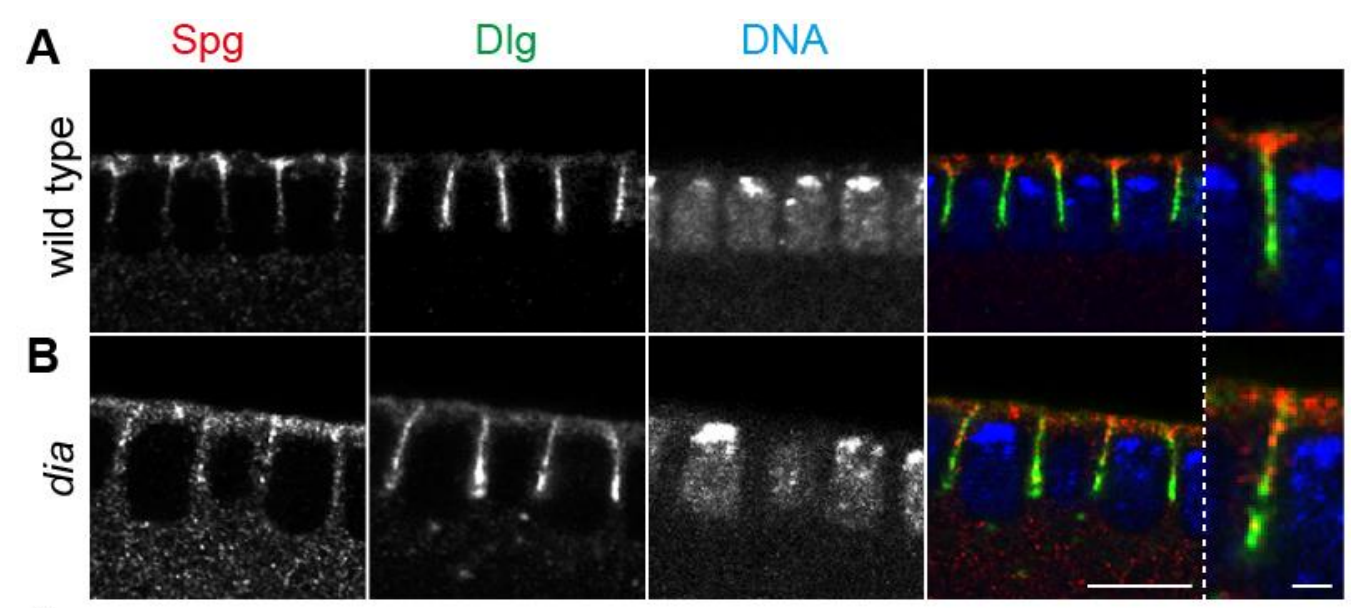

C

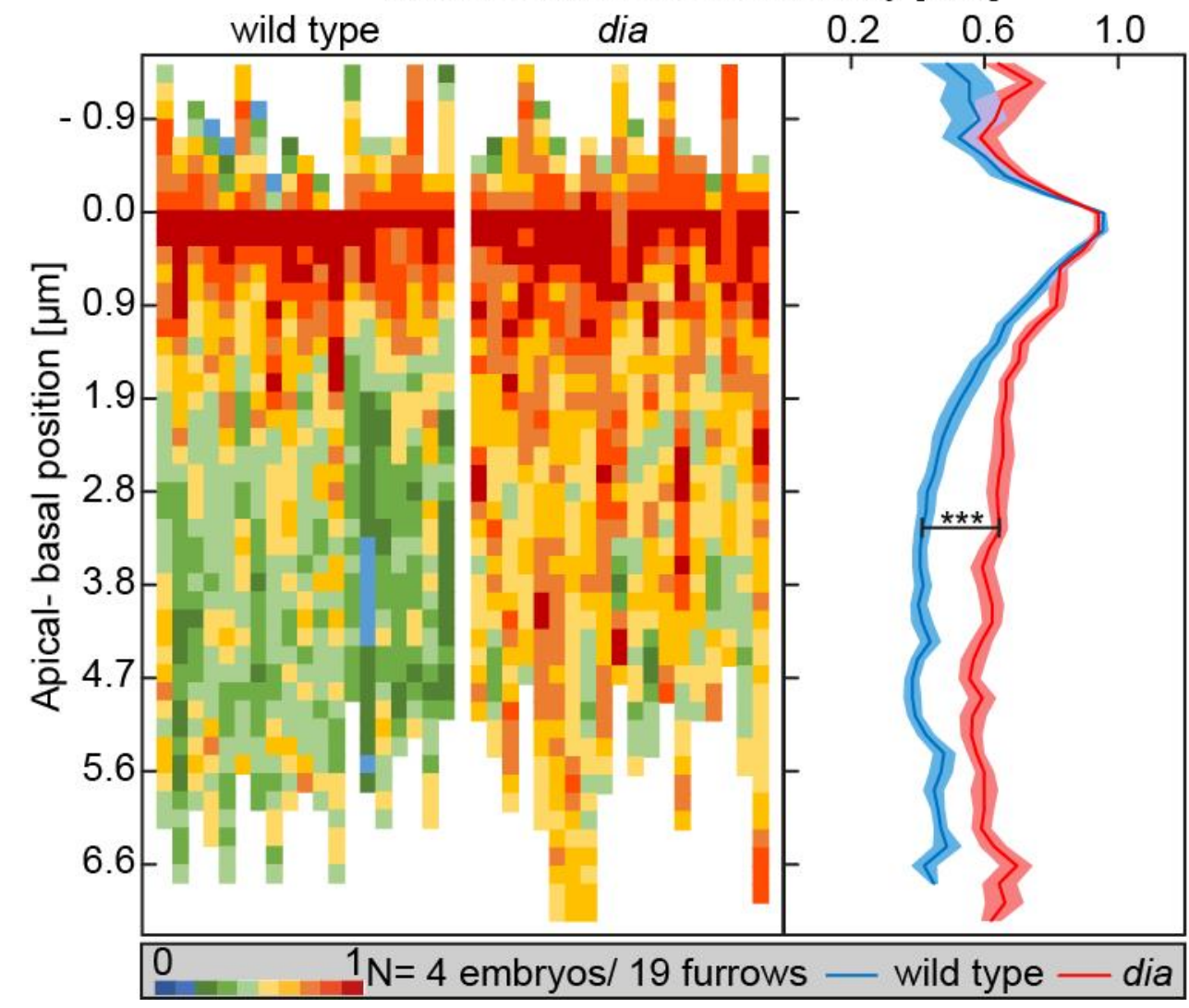

Figure 41 Subapical enrichment of Spg in wild type and dia embryos.

(A, B) Fixed cellularizing (A) wild type and (B) dia mutant embryos were stained against Spg (grey/red), Dlg (grey/green) and DNA (grey/blue). Merged images and zoom ins are shown in right panels. (C) Heat maps of relative fluorescence intensities of Spg signal measured along the apical-basal axis of cellularization furrows of six wild type and eight dia embryos as indicated. Each row reflects one cellularization furrow. Averages of relative fluorescence intensities of Spg signal in wild type (blue) and dia (red) embryos were plotted against the apical- basal position (right side). Filled regions indicate SEM. Scale bars $10 \mu \mathrm{m}$, zoom ins $2 \mu \mathrm{m}$.

As it was already visible in wild type, the heat maps showed subapical enrichment in wild type but also some protein could be found along the lateral membrane. The subapical enrichment was also detectable in dia embryos, 
however, like for Canoe, this enrichment was broader and relatively more protein could be detected along the lateral domain (Figure 41C, heat maps). This could be confirmed by the plot of the averages of the relative fluorescence intensities. Furthermore, the difference of relative fluorescence intensities at position $3.2 \mu \mathrm{m}$ at the lateral domain was highly significant $(\mathrm{p}<0.001)$.

Taken together, I could show, that the function of Dia is necessary for subapical localization of Armadillo, Bazooka and Canoe and Sponge and also for their restriction from the lateral domain leading to the hypothesis, that Dia is involved in the formation of the subapical domain. However, it is still unclear, how Dia could mechanistically act on the positioning of subapical cues and their restriction from the lateral domain.

\subsection{Dia and F-actin are enriched at the basal domain during cellularization}

\section{F-actin is not enriched at the subapical domain during early and mid- cellularization}

The cortex is built by filamentous actin underlying the plasma membrane and functions as a scaffold for proteins resulting in the possibility to form cortical domains. It was described before that F-actin is needed for subapical localization of Canoe and Bazooka, as perturbation of F-actin by injection of Latranculin A led to mislocalization of Canoe and Bazooka (Choi et al., 2013; Harris and Peifer, 2004). With this knowledge I examined the localization of Factin during cellularization to investigate if F-actin could directly or indirectly mediate the localization of subapical proteins. For this purpose, I stained fixed wild type embryos with fluorescently labeled Phalloidin which marks F-actin and Dlg (Figure 42A). During early cellularization, before nuclear elongation started, F-actin was still enriched at the actin caps and showed an enrichment at the tip of the invaginating furrow (Figure 42A, upper panel). With elongation of the furrow and flattening of the actin caps, the enrichment of $\mathrm{F}$ actin at the caps was lost, although an F-actin signal was still detectable along the apical and lateral membranes, whereas the enrichment at the basal furrow canal got even stronger with progressing membrane invagination (Figure 42A, panels 2-4). To analyze if F-actin localization is affected in dia, I stained fixed dia mutant embryos and stained for F-actin and Dlg (Figure 42B). During early cellularization, before nuclei elongation started F-actin was enriched at the apical caps and also at basal tips similar to wild type. The staining against Dlg 
showed, that the lateral marker spread into the basal domain as described before (Yan et al., 2013).

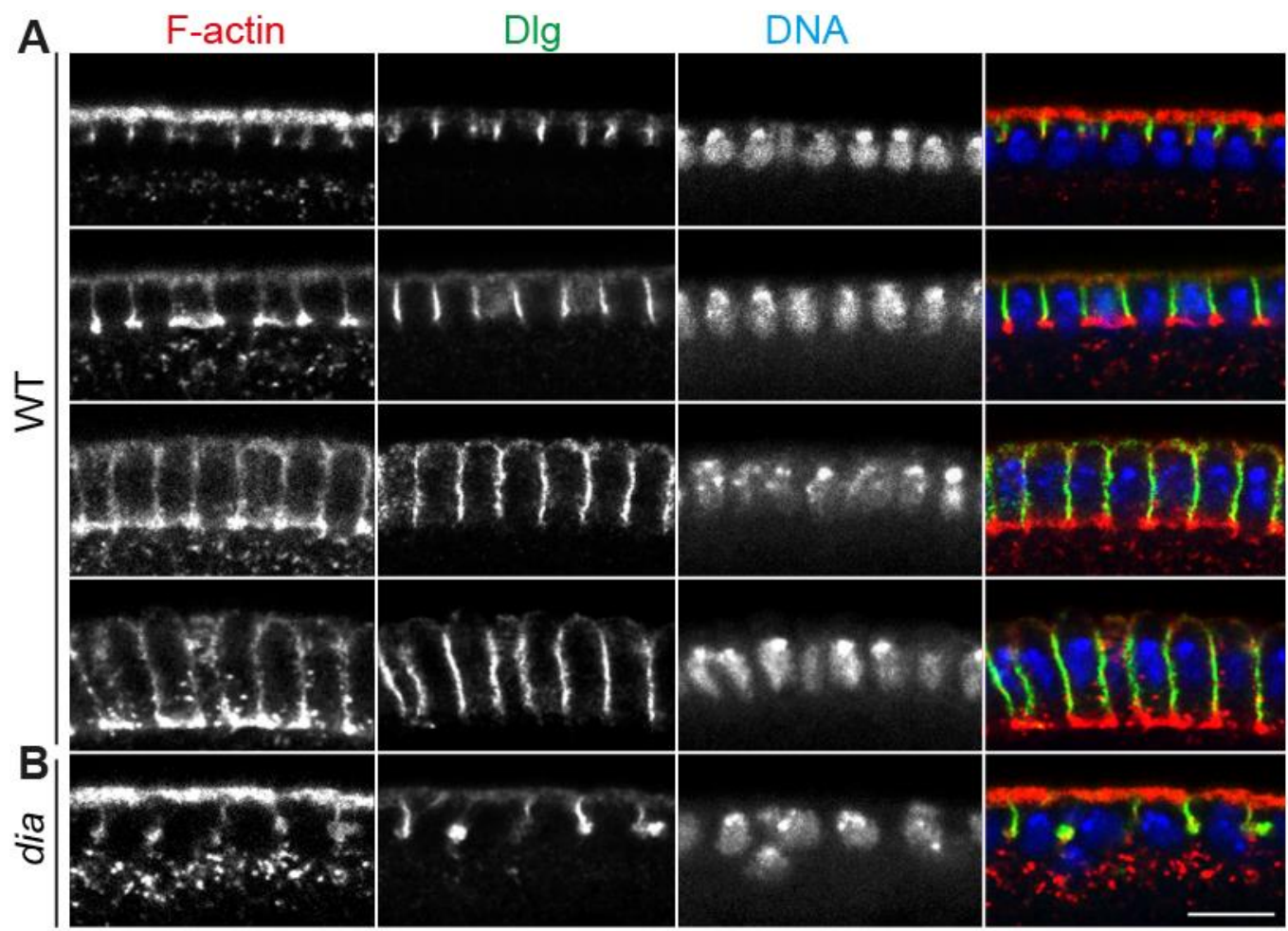

Figure $42 \mathrm{~F}$-actin is enriched at furrow canals during cellularization.

(A-B) Images of fixed embryos stained for F-actin (grey/ red), Dlg (grey/green) and DNA (grey/ blue). (A) Wild type embryos during early, mid and late cellularization staged by furrow length. (B) dia embryo during early cellularization. Scale bar $10 \mu \mathrm{m}$.

\section{Dia and Rho signaling are enhanced at furrow canals}

The nucleation and elongation of F-actin is mediated by a group of proteins called formins to which Dia belongs. To investigate if Dia could mediate the subapical localization of Canoe, Bazooka and Armadillo, I stained fixed wild type embryos against Dia and Dlg (Figure 43). During mid cellularization (Figure 43, upper panel) Dia was enriched at the basal domain, like F-actin. Although Dia was visible along the whole furrow, a subapical enrichment could not be detected. During the end of cellularization, a subapical enrichment of Dia got visible (Figure 43, lower panel). It was described before, that Dia shows an active state in which it nucleates and elongates F-actin and an autoinhibited state (Großhans et al., 2005; Li and Higgs, 2003). However, the antibody against Dia did not discriminate between the two forms, so it was still possible, that an enrichment of active Dia at the subapical domain mediates subapical enrichment of Canoe, Bazooka and Armadillo during early and mid-cellularization. 


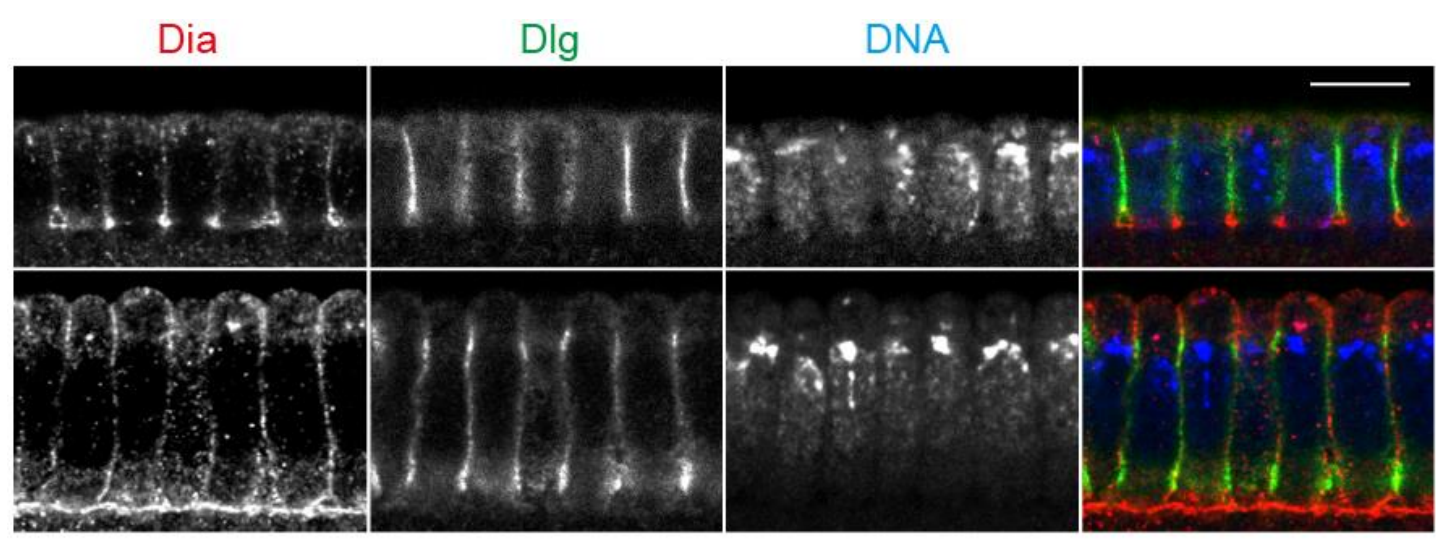

Figure 43 Dia is enriched at furrow canals during cellularization.

Images of fixed wild type embryos during mid (upper panel) and late cellularization (bottom panel) stained for Dia (grey/red), Dlg (grey, green) and DNA (grey/ blue). Scale bar $10 \mu \mathrm{m}$.

To investigate the localization of active Dia, I overexpressed a dominant active version of Dia with deleted C- and N-terminus, tagged to GFP. The construct was driven by a maternal Gal4 driver line. However, I could only detect a salt a pepper like signal in the embryo with some cells showing GFP signal and others not. A specific signal could not be detected (data not shown). To circumvent these problems, I made use of an indirect assay to detect the localization of active Rho1, that could locally activate Dia. For this assay the Rho binding domain (RBD) of Anillin was N-terminally tagged with dtTomato, named as Rho-sensor, expressed under the control of a nanospromoter. Although the nanos-promoter is germline specific, the leakage of this expression system made it possible to detect the Rho-sensor also in somatic cells. It was described before, that the localization of the RBD is likely to reflect the localization of an active protein with RBD (Abreu-Blanco et al., 2014). Figure 44 shows the dynamics of the Rho-sensor during Mitosis 13 (left panel), early cellularization (middle panel) and late cellularization (right panel) imaged in living embryos. The orthogonal views of $z$-stacks are shown in the bottom panels. During mitosis 13, the Rho sensor could be detected along the whole metaphase furrow. Whereas the Rho sensor was highly enriched at the basal domain during early and late cellularization in interphase 14. Like F-actin and Dia the RBD-sensor could also be detected along the lateral and apical domains. A subapical enrichment could not be detected.

Taken together, it is not likely that Dia and/or F-actin play a direct role in subapical localization of marker proteins, as no subapical enrichment of Dia or F-actin could be detected before late cellularization. Thus, it seems that Dia 
and F-actin have an indirect permissive function for the localization of subapical cues.

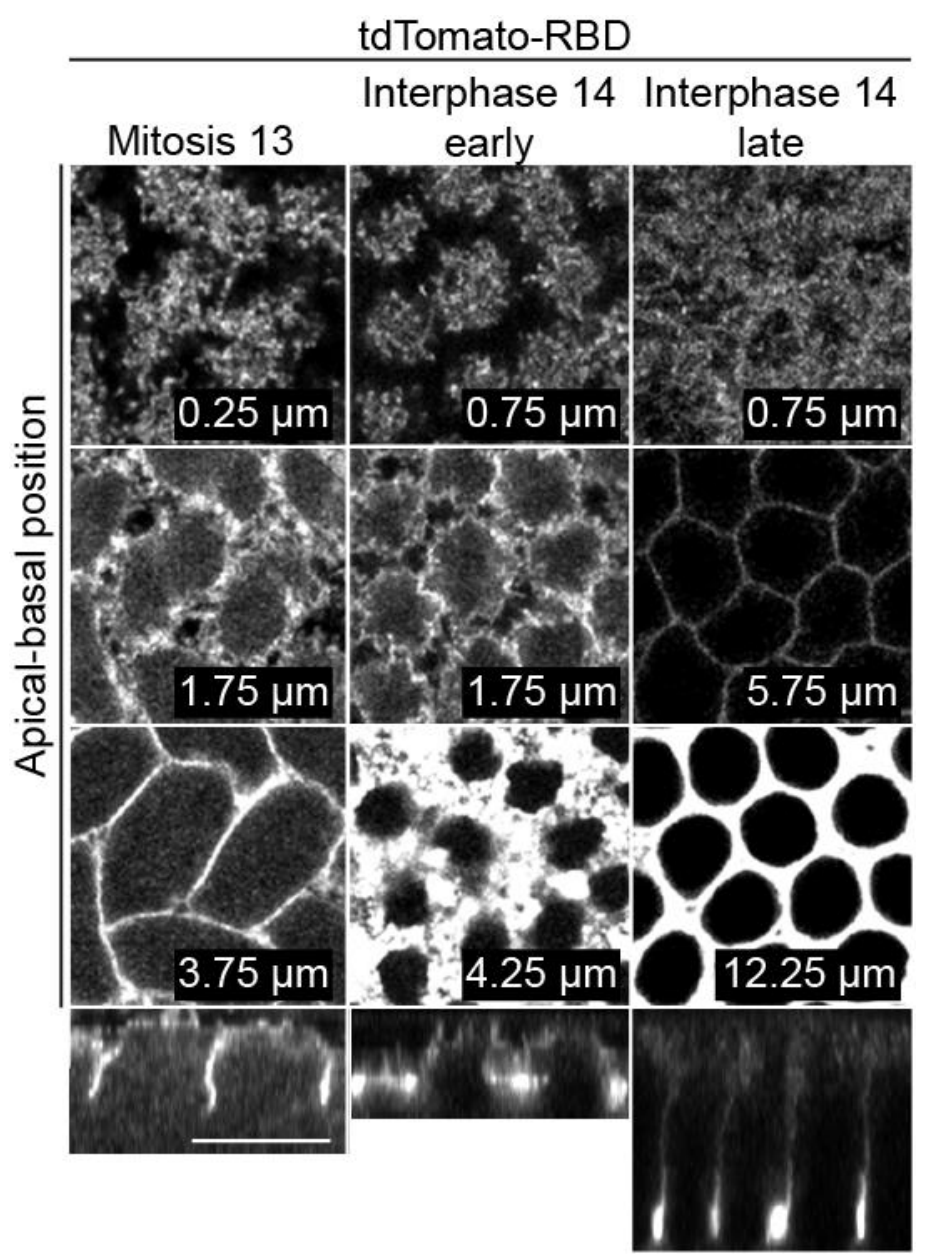

Figure 44 Dynamics of the Rho binding domain.

Images of living embryos expressing the Rho binding domain labeled with tdTomato during Mitosis 13 (left panel), early cellularization (middle panel) and late cellularization (right panel). Top views with apical-basal position as indicated in lower right. Corresponding orthogonal views are shown in bottom panel. Scale bar $10 \mu \mathrm{m}$.

\subsection{Diffusion of Canoe and integral membrane proteins is not affected in dia}

The mobility of Canoe during cellularization does not change in dia

The actin cortex can influence the lateral diffusion of cortical proteins and cortical domains can be separated by diffusion barriers (reviewed in Honigmann and Pralle, 2016). As shown before, F-actin was still detectable at the invaginating furrows in dia mutants although the F-actin seemed somehow disorganized. Furthermore, the barrier between lateral and basal domain is not functionable anymore in dia mutants, with lateral proteins also leaking into the basal domain (Yan et al., 2013). To test if cortical Canoe at the subapical domain shows differences in dynamics in wild type compared to dia mutants, 
I quantified the recovery of fluorescence after bleaching in cellularizing embryos expressing CanoeYFP. By measuring the fluorescence intensities along the time after bleaching, the dynamics of the protein could be detected and investigated if different cortical properties in wild type versus dia mutant embryos could influence these dynamics. Figure 45 shows fluorescence recovery after photobleaching (FRAP) experiments in living CanoeYFP expressing wild type and dia mutant embryos.
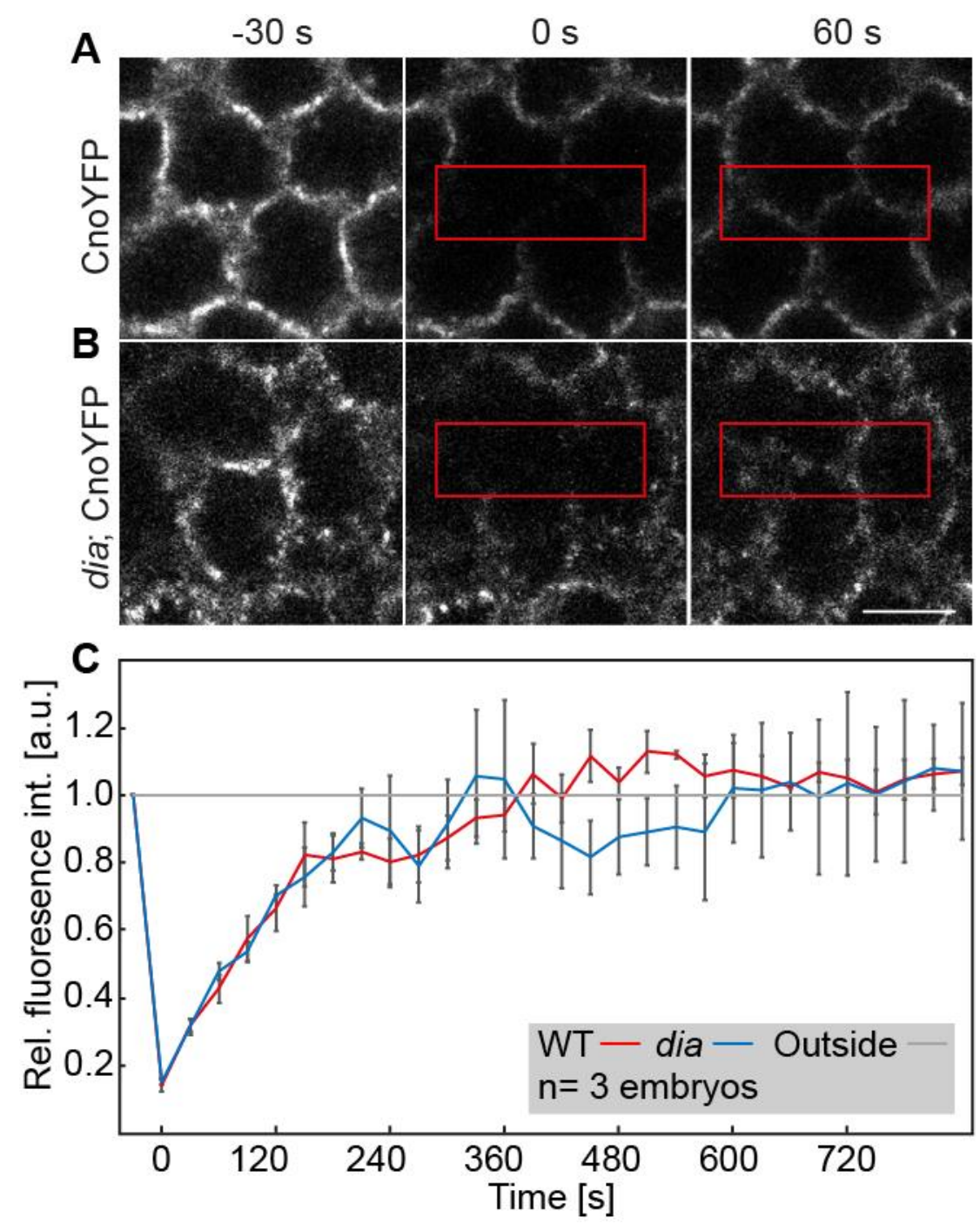

Figure 45 Recovery of CanoeYFP after bleaching in wild type and dia during cellularization.

(A-B) FRAP in living embryos expressing CanoeYFP. Images from movies of (A) wild type and (B) dia embryos. Timepoints are as indicated and the region of interest is marked by a red rectangle. (C) Averaged relative fluorescence intensities before and after FRAP of CanoeYFP in 3 wild type (red line) and dia (blue line) embryos. Fluorescence intensities of furrows in the region of interest are normalized to the peak and to fluorescence intensities of furrows outside of the region of interest (grey line) to correct for photobleaching. Scale bar $5 \mu \mathrm{m}$.

The bleaching was conducted $10 \mathrm{~min}$ after start of cellularization in the subapical region. To be able to follow the bleached region during the dynamic process of cellularization I imaged z-stacks with three slices and with a step 
size of $1.5 \mu \mathrm{m}$ in an interval of 30 seconds. In Figure 45A, B top views of wild type and dia embryos before, directly after and $60 \mathrm{~s}$ after bleach of several furrows were marked. The images show, that already after 60 seconds a high rate of recovery was reached. To quantify the fluorescence recovery, I measured the fluorescence intensities in the regions of interest of three embryos per genotype before and after bleaching, normalized to its peak and to fluorescence intensities outside of the bleached region to correct for photobleaching during imaging. The averages of relative fluorescence intensities were plotted against the time (Figure 45C). A significant difference in fluorescence recovery of CanoeYFP was not detectable between wild type and dia embryos. It seems, that dia did not influence the dynamics of CanoeYFP during the process of cellularization.

\section{The diffusion of 117GFP is not affected in dia preblastoderm embryos}

As also the lateral diffusion of integral membrane proteins is regulated by their connection to the underlying actin cortex (reviewed in Kusumi et al., 2005), I wanted to test if this is affected in dia embryos. For this examination I made use of the integral membrane protein 117, tagged with GFP and expressed under the control of the ubiquitin-promoter in wild type and dia mutant background. To get further insight into a more general influence of Dia on the cortex and the lateral diffusion of membrane proteins, I tested the recovery of 117GFP in preblastoderm embryos in which only one cortical domain is existing. Figure 46A shows top views of wild type (upper panel) and dia mutant embryos in preblastoderm expressing 117GFP before and after bleaching. The bleached region is marked with blue and red circles as indicated. To evaluate the recovery speed of the 117GFP signal, I measured the fluorescence intensity of the region of interest in seven wild type and six dia embryos, normalized it to its peak and to the outside to correct for photobleaching. The averages were plotted against the time as shown in Figure 46B. The recovery of 117GFP signal after bleaching did not differ significantly between wild type and dia embryos. It seems, that general lateral diffusion of integral membrane proteins is not affected in dia mutants, at least not in preblastderm embryos with only one cortical domain. 
A

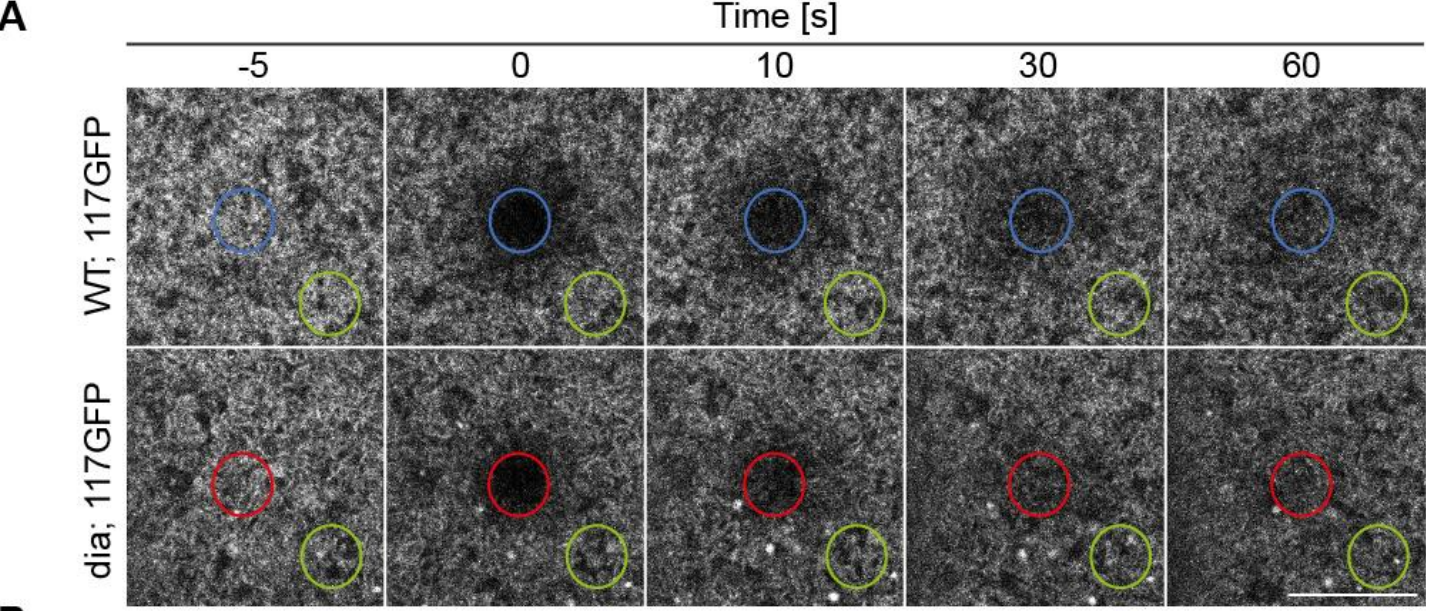

B

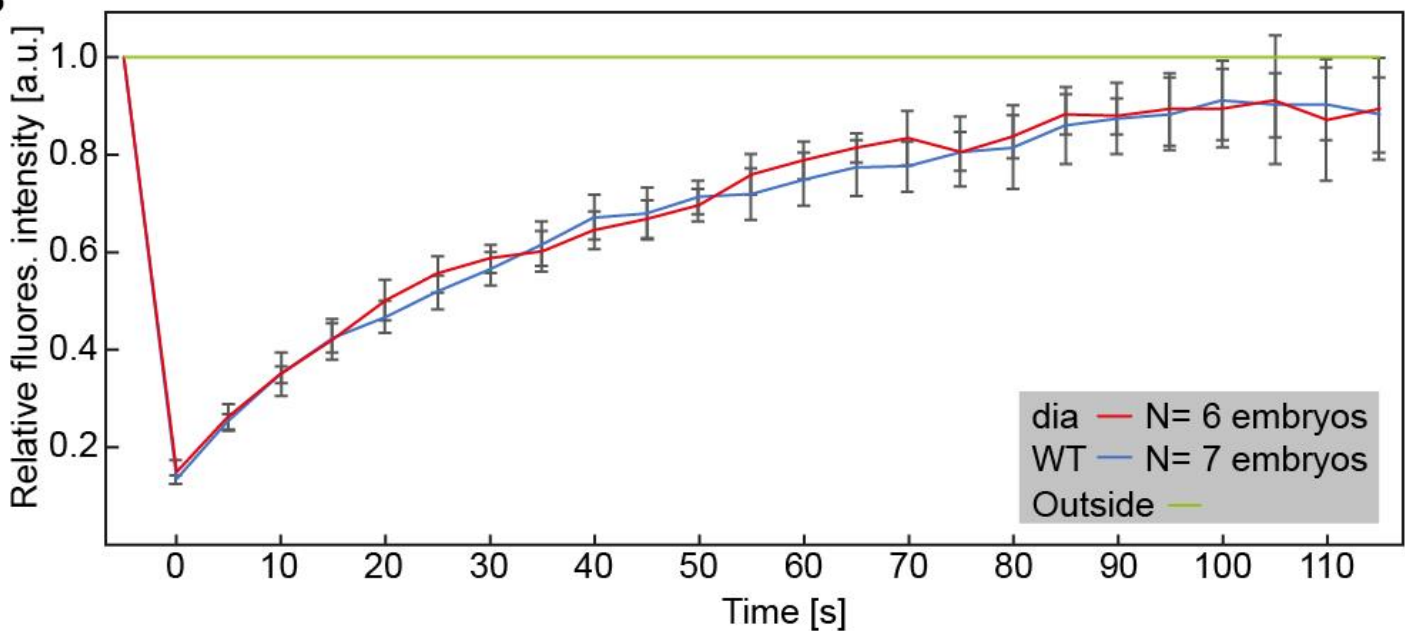

Figure 46 Recovery of the integral membrane protein 117GFP in wild type and dia preblastoderm embryos.

(A-B) FRAP in living embryos expressing 117GFP. (A) Images from movies of wild type (upper panel) and dia (lower panel) embryos. Timepoints are as indicated and the region of interest is marked by a blue (wild type) and red ( $\mathrm{dia}$ ) circle. (B) Averaged relative fluorescence intensities before and after FRAP of 117GFP in 3 wild type (blue line) and dia (red line) embryos. Fluorescence intensities in the region of interest were normalized to their peak and to a region outside of the bleached region (green) to correct for photobleaching. Scale bar $10 \mu \mathrm{m}$.

\subsection{The effect of endocytosis on the formation of the subapical domain is dispensable}

\section{Subapical localization of Bazooka does not depend on endocytosis}

It was reported, that Dia could stabilize the actin cortex by the formation of linear actin filaments and by this also preventing endocytosis, as in dia mutants an extended endocytic activity could be detected (Yan et al., 2013). In contrast, branched F-actin, produced by Arp $2 / 3$ could promote endocytosis by stabilizing the neck of the forming endocytic bud (reviewed in Robertson et al., 2009). Furthermore, it was described that endocytosis provides the 
invagination of the cellularization furrow by providing membrane material (Fabrowski et al., 2013; Lecuit and Wieschaus, 2000).

A
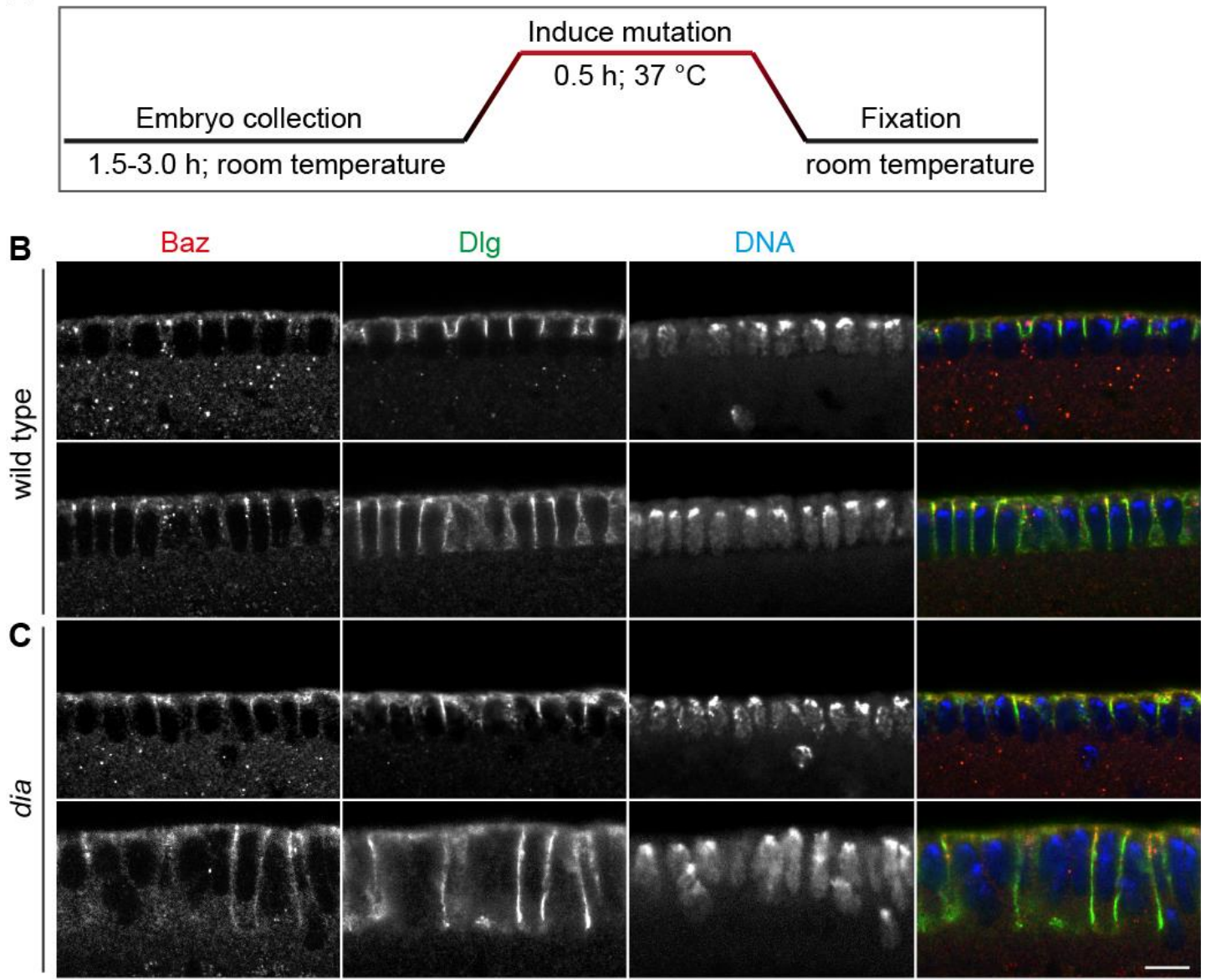

Figure 47 Subapical enrichment of Baz is not perturbed in shi embryos.

(A) Scheme showing the process of induction of shi mutation. (B-C) Images of fixed wild type (B) and (C) shi embryos stained for Baz (grey/ red), Dlg (grey/green) and DNA (grey/ blue). Merged images are shown in right panel. Scale bar $10 \mu \mathrm{m}$.

To test if the defective formation of cortical domains in dia mutants is due to destabilization of the actin cortex and extended endocytosis, I made use of a temperature sensitive mutation in the gene shibire (shi). Shibire (Dynamin in vertebrates) is needed for the final scission of vesicles during endocytosis and without Shibire, cellularization cannot take place because membrane elongation stops as not enough membrane is provided (Fabrowski et al., 2013). To induce the mutation during early cellularization, I collected wild type and shibire embryos for 1.5-3.0 hours at room temperature and then heat shocked both collections for 0.5 hours at $37^{\circ} \mathrm{C}$ in a water bath. The embryos were covered by water to prevent drying-out. Afterwards, the embryos were fixed following standard methods as described before (Figure 47A). Early and mid cellularizing wild type embryos showed subapical Bazooka enrichment and 
Dlg staining the lateral domain (Figure 47B). Embryos in which the mutation of shibire was induced before or at early cellularization displayed missing furrows and multiple nuclei in between two cellularization furrows. However, restriction of Bazooka from the lateral domain seemed not to be perturbed in furrows of early and late cellularizing embryos (Figure 47C), concluding that endocytosis did not have a direct function on formation and maintenance of the subapical domain.

\section{Rab proteins do not show a subapical enrichment during cellularization}

Rab proteins belong to the family of GTPases and are known as regulators of intracellular transport by vesicle trafficking (reviewed in Zerial and McBride, 2001). As I investigated the role of endocytosis for the formation of the subapical domain, I analyzed the localization of several YFP-tagged Rab proteins during early embryogenesis. For this analysis I made use of fly lines which control the YFP-tagged Rab proteins endogenously (yRab lines; Dunst et al., 2015). An interesting candidate was Rab35 that regulates the terminal steps of endocytosis during cell division in Drosophila S2 cells by controlling Septin and the subcellular distribution of $\mathrm{PIP}_{2}$ (Kouranti et al., 2006). Furthermore, it was found to regulate the formation of filopodia in S2 cells and the assembly of actin filaments in the process of bristle development (Zhang et al., 2009). To analyze the localization of yRabs, I stained fixed embryos expressing yRabs with the GFPbooster to detect the YFP-tagged Rab and Dlg and Slam to mark lateral and basal domains. During the course of cellularization I could detect yRab35 along all domains with no specific enrichment at the subapical domain (Figure 48). During late cellularization, I could detect Rab35 positive compartments marking endocytic vesicles as described before for gastrulation (Jewett et al., 2017). 


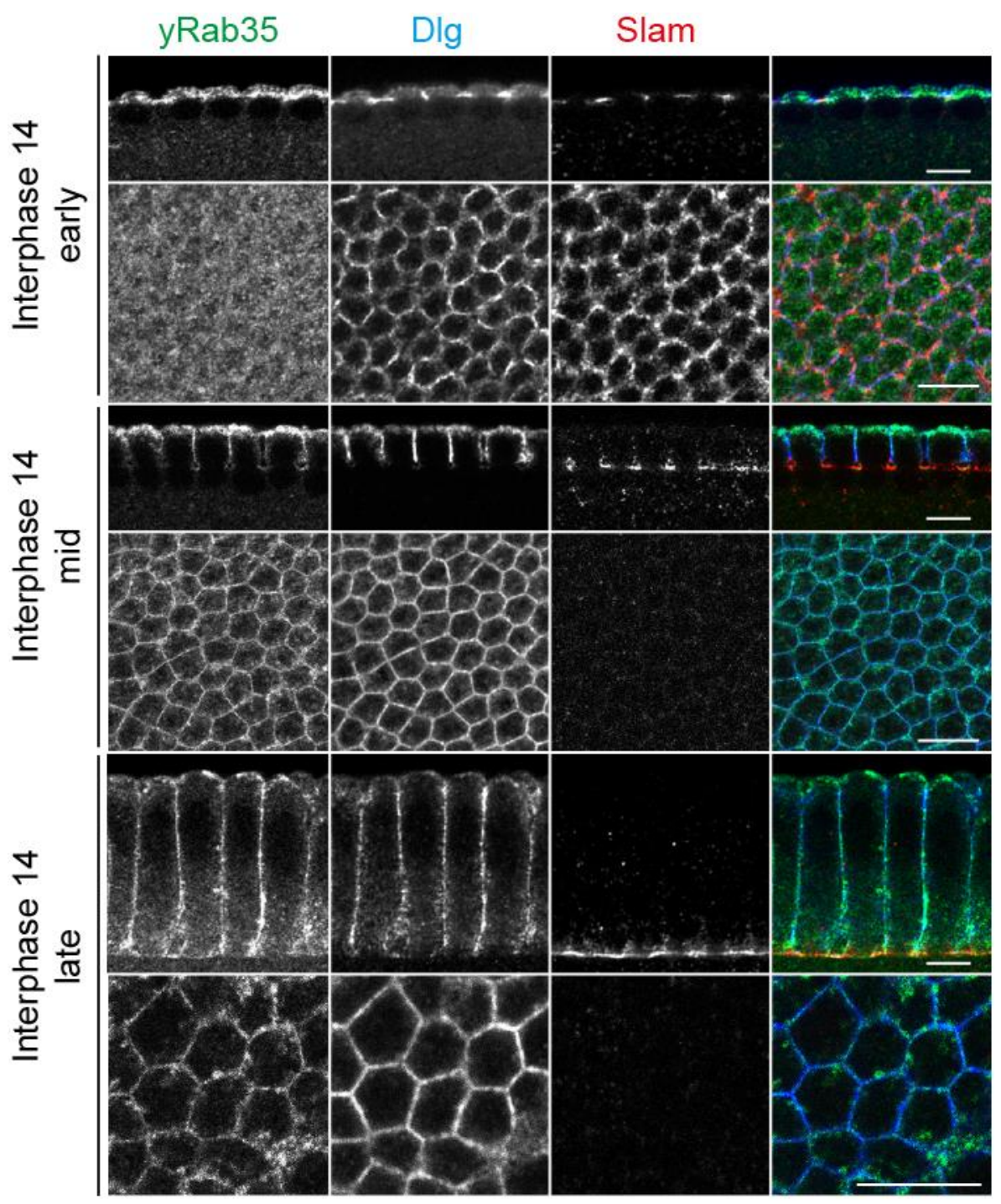

Figure 48 yRab35 localizes to all cortical domains during cellularization.

Fixed cellularizing wild type embryos expressing YFP-Rab35 stained by GFP booster (grey/ green), Dlg (grey, blue) and Slam (grey/ red). Stages are as indicated. Merged images are shown in right panel. Upper panels show side views and lower panels top views of representative lateral positions. Scale bars $10 \mu \mathrm{m}$.

Rab23 that was described to play a role in the activation of the planar cell polarity and inhibits hair formation at positions outside of the distal vertex (Pataki et al., 2010), was also found to be expressed during early embryogenesis. I stained embryos expressing YFP-Rab23 as explained before Figure 49. During mitosis 13 YFP-Rab23 localized to the apical and the lateral domain of metaphase furrows and during interphase 13 it was found at actin caps. During celullarization I could detect Rab23 puncta along all cortical domains without any specific enrichment. 


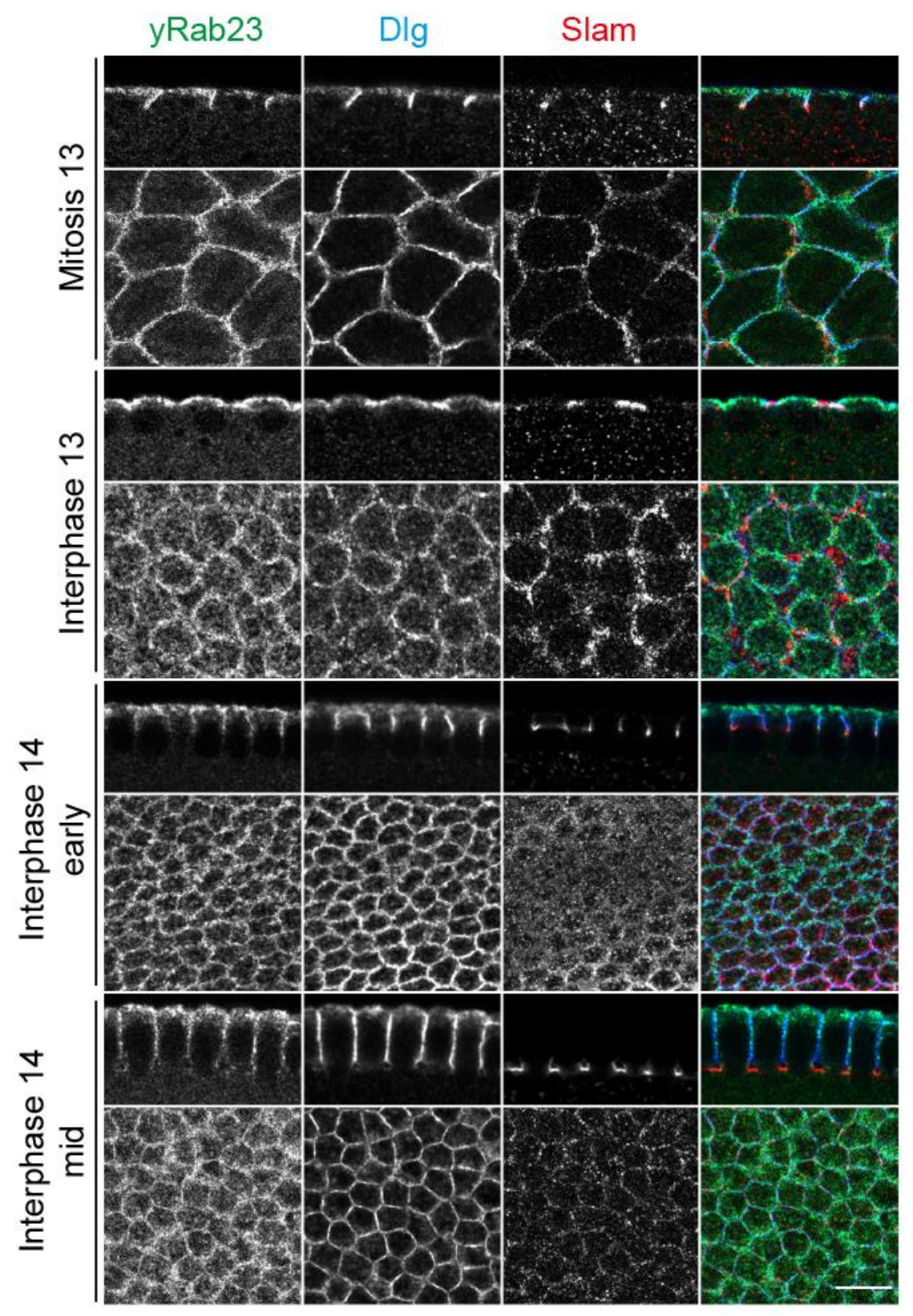

Figure 49 Localization of yRab23 during early embryogenesis.

Fixed cellularizing embryos expressing YFP-Rab23 stained with GFPbooster (grey/ green), Dlg (grey/ blue) and Slam (grey/red). Stages are as indicated. Upper panels show side views, lower panels top views of a representative lateral position. Merged images are shown in right panels. Scale bar $10 \mu \mathrm{m}$.

\subsection{Cortical F-actin is polarized and this organization depends on Dia}

\section{Dia is enriched at the rims of actin caps}

The function of Dia and F-actin on the formation of cortical domains seems not to be direct but more permissive, allowing the marker proteins to localize to their associated domains. As I could show, that F-actin is still present in dia mutants, the mislocalization of the marker proteins in dia was not due to 
missing F-actin. However, the F-actin of the cellularization furrow seemed to be loosened up and somehow misorganized in dia compared to wild type during cellularization (Figure 42B). To test if the actin cortex shows an organization or polarization, I stained fixed wild type embryos against Dia and F-actin (Figure 50). Dia is known to bind to the barbed or plus-ends of the actin filaments where they are elongated by Dia (Moseley et al., 2004). During interphases of syncytial blastoderm, F-actin marked the actin caps and also Dia was visible at the actin caps but did not show a complete overlap with Factin and seemed more enriched at the rims of the actin caps (Figure 50).

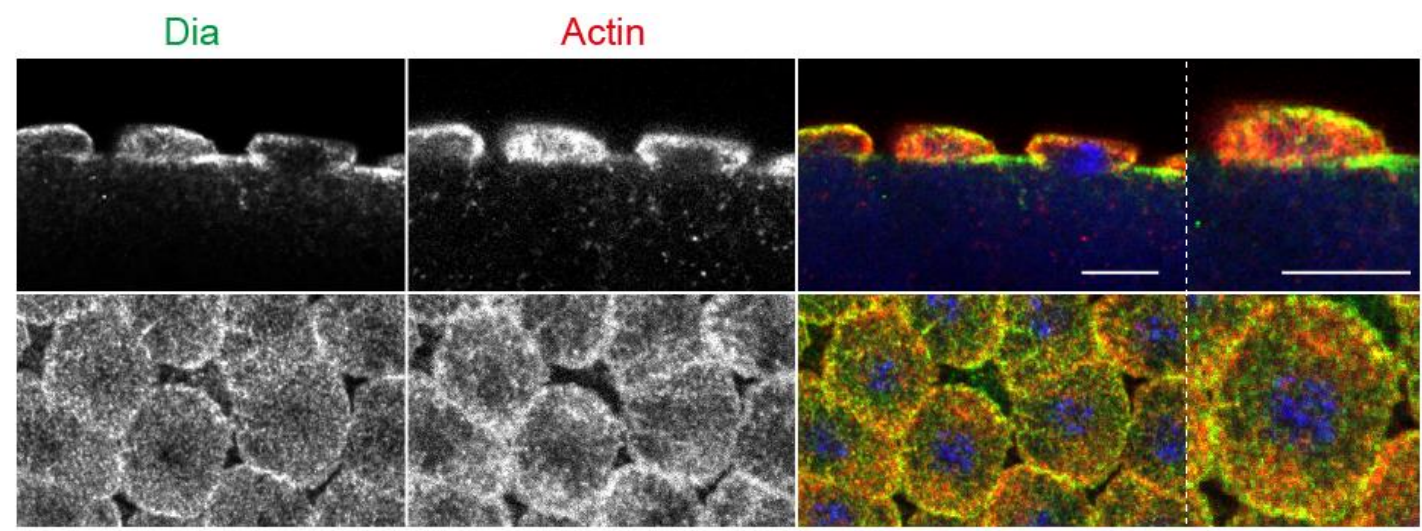

Figure 50 Dia localization to actin cap rims in syncytial blastoderm wild type embryos.

Fixed wild type embryos during a syncytial blastoderm interphase stained against Dia (grey/ green), F-actin (grey/ red) and DNA (blue). Upper panels represent the side view and lower panels the corresponding top view. Merged images and zoom ins are shown in right panels. Scale bars $10 \mu \mathrm{m}$.

\section{Cpa localizes to enriched barbed ends of actin filaments at rims of caps}

As I also intended to analyze the cortical organization in dia mutants, I made use of another plus-end binding protein, named Capping protein alpha (Cpa) (Isenberg et al., 1980). The capping protein heterodimer consisting of Capping protein alpha and beta binds to the barbed ends of actin filaments, inhibiting the addition of actin monomers to the filament (Isenberg et al., 1980). Recently, it was shown, that the apical cortical actin network of epithelial cells during ventral furrow formation is organized in a way, that the plus ends are enriched at the junctions whereas the minus ends are in the medial part, playing a role in Myosin organization and contraction and following apical constriction (Coravos and Martin, 2016; Lv and Großhans, 2016).

To confirm, that the cortical F-actin also shows an organization in syncytial embryos during interphase 13, mitosis 13 and early interphase 14, I stained fixed wild type embryos against Cpa and F-actin (Figure 51). Indeed, during 
interphase 13, Cpa localized to the actin cap without complete overlap with Factin, but enrichment at the rims of the caps (Figure 51, upper panels).

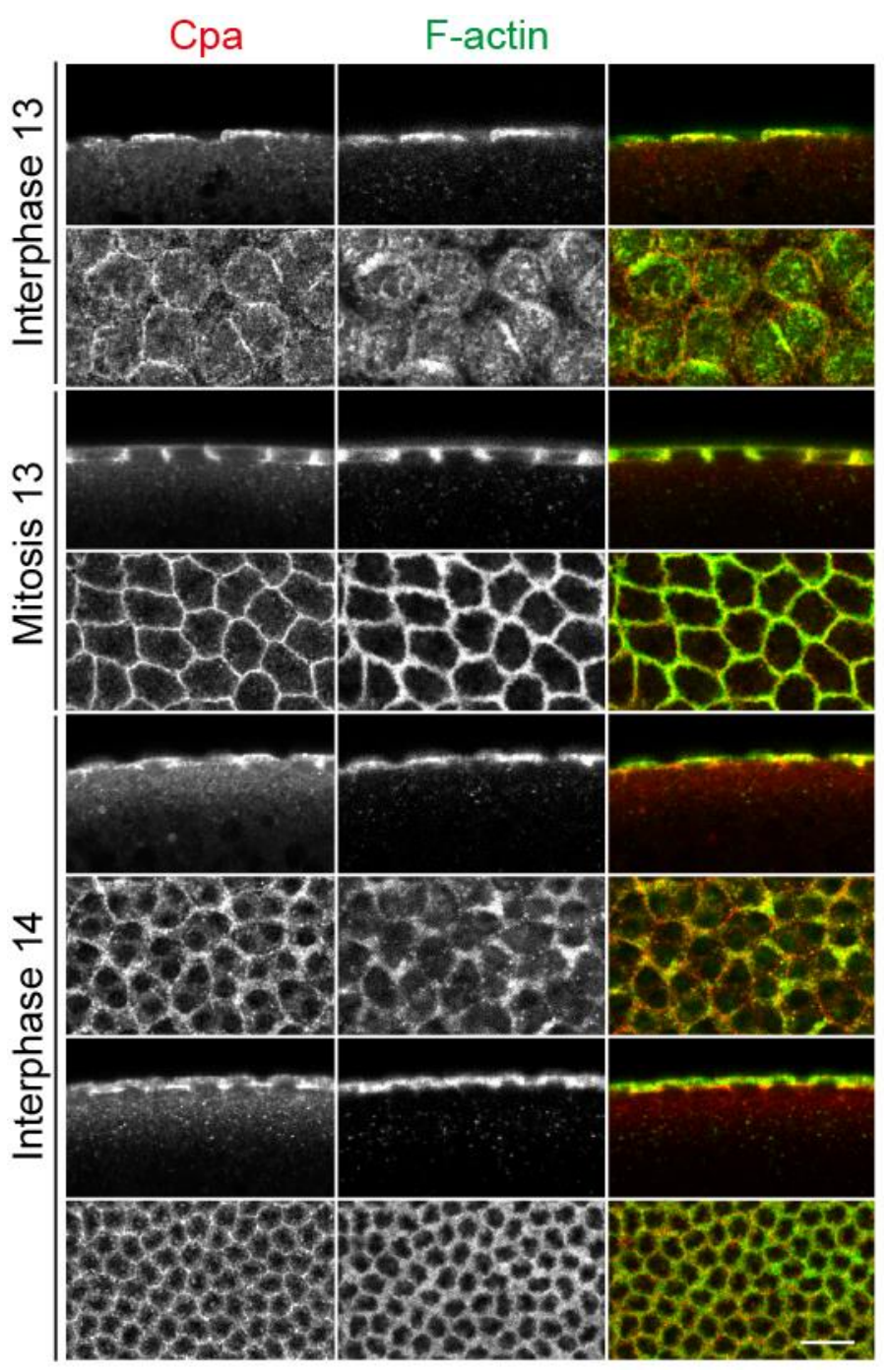

Figure $51 \mathrm{Cpa}$ enrichment at the rims of actin caps during syncytial blastoderm in wild type embryos.

Staining against $\mathrm{Cpa}$ (grey/red) and F-actin (grey/green) during interphase 13, mitosis 13 and interphase 14 in wild type embryos as indicated. Scale bar $10 \mu \mathrm{m}$.

In mitosis 13, Cpa localized along the metaphase furrow without an enrichment at a specific domain (Figure 51, middle panels), and during early interphase 14, the localization to the rims of the actin caps got even more obvious (Figure 51, lower panels). The shown data indicated, that the cortical F-actin in caps during syncytial development is organized with F-actin plus ends pointing to the rims of the actin caps.

\section{The separation of cap and intercap domain is functional in dia mutants}

Before I investigated the localization of Cpa in dia mutant embryos, I wanted to clarify if the separation of cap and intercap domains is functional in dia 
mutants as the domain separation is perturbed during cellularization. For this I stained F-actin in wild type and dia embryos expressing MyoII tagged with 3x GFP (Figure 52). In both genotypes the domains were clearly separated and did not show any overlap, although intercap staining by MyoII seemed to be more punctate and less continuous in dia mutants (Figure 52B).

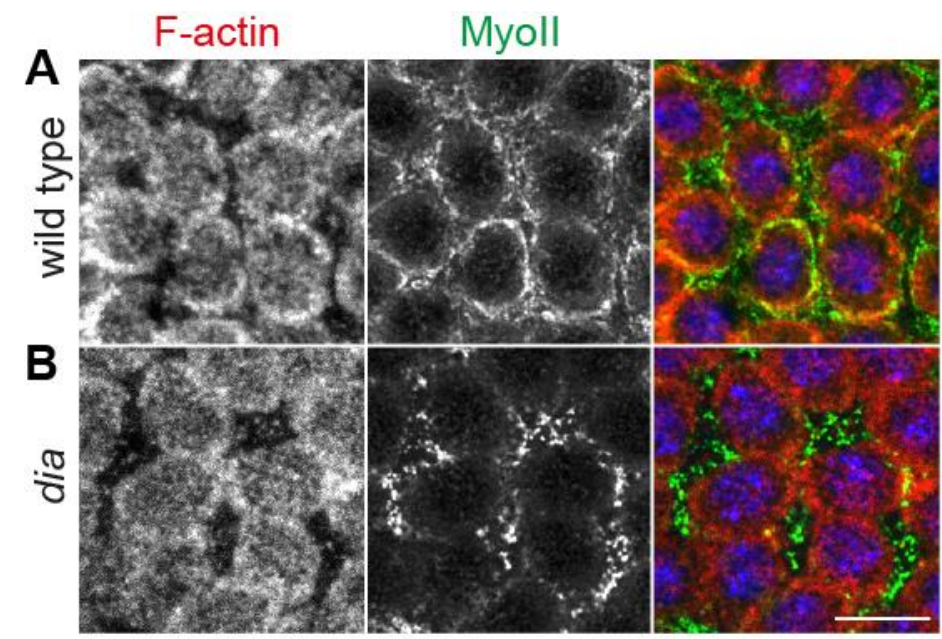

Figure 52 The separation of cap and intercap domains in wild type and dia embryos.

Top views of fixed interphase embryos during syncytial blastoderm stained for F-actin by Phalloidin (grey/ red), MyoII by GFPbooster (grey/ red) and DNA (blue). Genotypes are (A) wild type and (B) dia. Scale bar $10 \mu \mathrm{m}$.

\section{Cpa is less enriched at actin cap rims of dia embryos}

Since I could show, that the actin caps of syncytial embryos show an organization of the cortical actin with plus ends to the rims, I next compared this organization in wild type and dia mutant embryos. For this I stained wild type and dia mutant embryos against Cpa and F-actin (Figure 53). For optimal comparison, I used dia mutant embryos, expressing a GFP-tagged Myosin and stained both genotypes in the same tube and mounted them on the same slide. The mutant embryos could be detected by GFP fluorescence. In early interphases 12 and 13 Cpa could again be detected enriched at the rims of actin caps as shown by side and top views in wild type embryos (Figure 53A). In dia mutants, this enrichment seemed to be broader and less stringent than in wild type (Figure 53B). Especially in the side views Cpa seemed to be distributed equally over the whole actin cap. Concluding from this staining, I could show, that the organization of the cortical actin seemed to be loosened up in dia mutants.

To confirm the effect of Dia on the organization of the cortical actin network, which I could show by the staining, I quantified the enrichment of the signal 
of the staining against Cpa at the rims of the actin caps (Figure 54). For this purpose, I measured the fluorescence intensity along a line with a width of 10 pixel which was placed over the rim of the actin cap (Figure 54A). I measured in total 98 rims of three wild type and 108 rims of three dia embryos, which were in interphases 11-13.

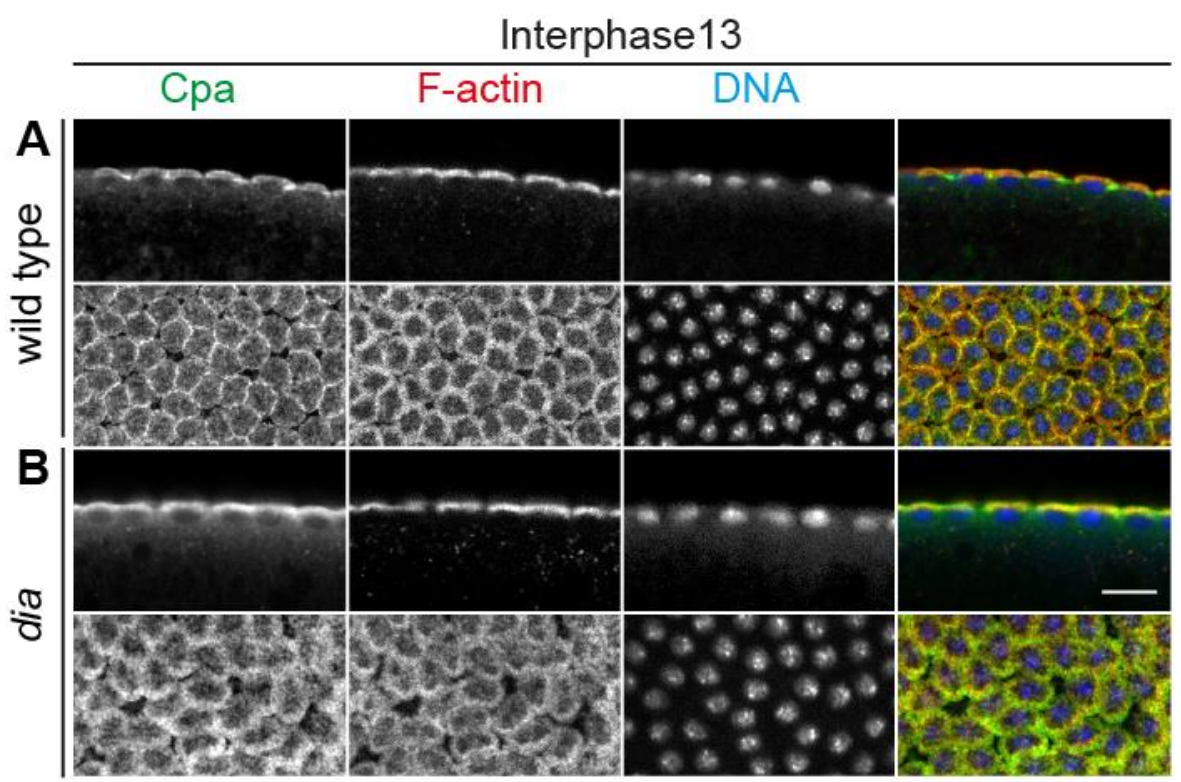

Figure 53 The Cpa signal at the rim of actin caps gets broader in dia mutants.

Staining against Cpa (grey/green), F-actin (grey/red) and DNA (grey/blue) of fixed wild type and dia mutant embryos during syncytial blastoderm interphases. Scale bar $10 \mu \mathrm{m}$.

The stage was estimated by the size of nuclei and actin caps. To plot the measurements as a graph in a comparable manner, I normalized every measurement to its peak and calculated the mean value for each embryo (Figure 54B). From this data, it gets clear, that the measurements of all three embryos per genotype were comparable, so that the stage of the syncytial blastoderm embryo did not influence the enrichment of Cpa at the rim. Furthermore, the data could show that the Cpa enrichment at the rims of the caps is less prominent in dia mutants, with a much broader distribution of Cpa at the rims of actin caps. To confirm, that the difference in Cpa distribution was significant, I measured the width of the curves of every measured cap of wild type and dia at a relative fluorescence intensity of 0.8 and plotted the width as box plots (Figure 54C). The average width of wild type was around $0.6 \mu \mathrm{m}$ compared to $1.5 \mu \mathrm{m}$ in dia and the difference of both was highly significant with a p-value of less than 0.001 . 

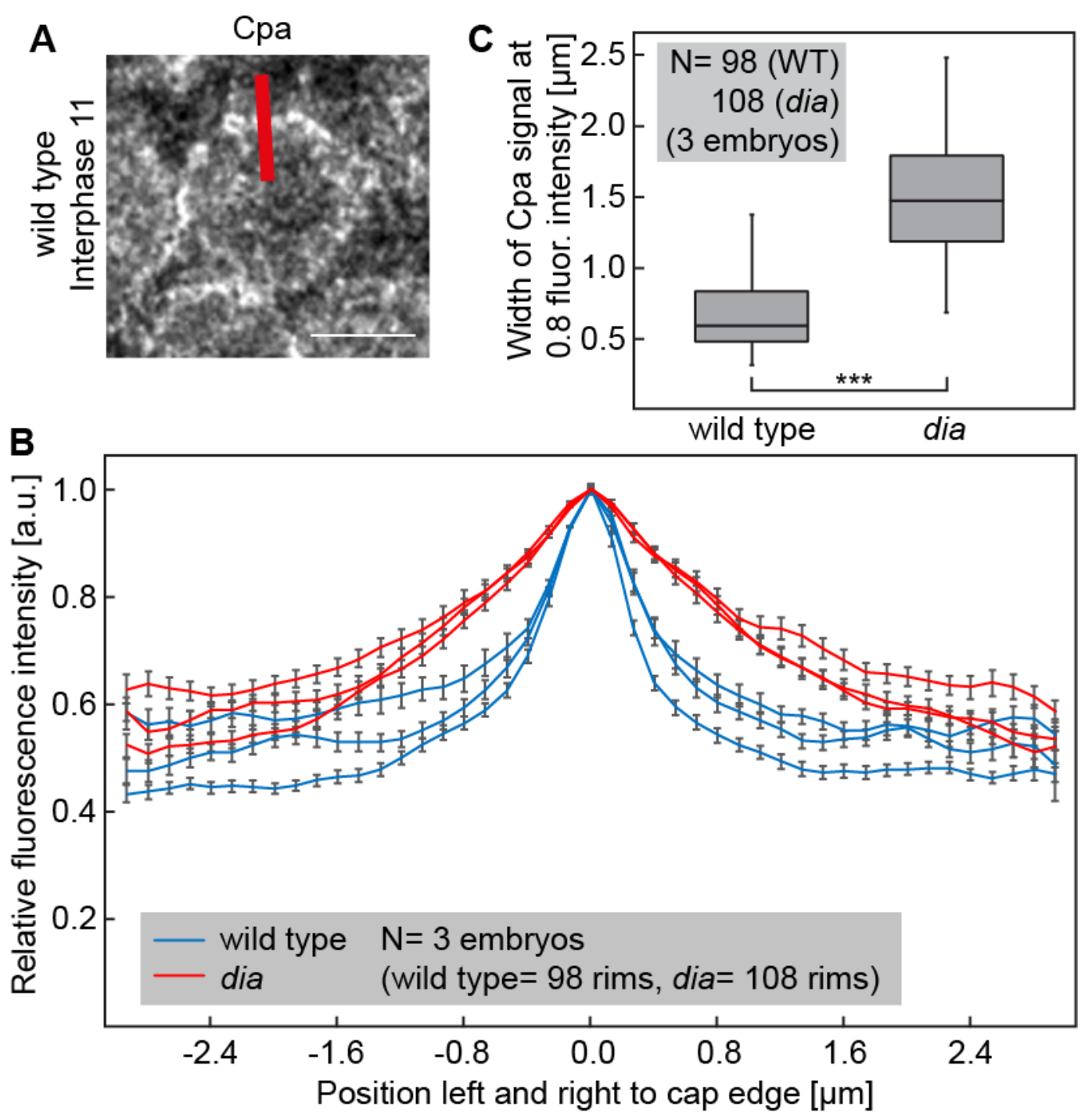

Figure 54 Cpa enrichment at cap edges in wild type and dia syncytial blastoderm embryos.

(A) Interphase 11 wild type embryo stained against Cpa. The red line marks how measurement of fluorescence intensity along the rim of actin caps was performed. (B) Averages of relative fluorescence intensities of three wild type (blue line) and dia (red line) embryos were plotted against the position left and right to the cap edge. Measured fluorescence intensities were normalized against its maximum and aligned to 1. Error bars represent SEM. (C) The width of the curves was measured at a relative fluorescence intensity of 0.8 for all single measurements (wild type $=98, d i a=108$ ) and plotted as boxplots for both genotypes. $\mathrm{P}<0.001$. Scale bar $5 \mu \mathrm{m}$.

In order to circumvent the arbitrary selection of a fluorescence intensity at which I measured the width of the curve, I included another method to describe the form of the curves of Cpa enrichment in wild type and dia mutants. For this I selected the averaged data from the three wild type and dia embryos, which are shown in Figure 54B, from -1.6 to $1.6 \mu \mathrm{m}$, mirrored the data at an $x$-value of 0 and then fitted exponential curves to the six wild type and dia curves, which are shown in Figure 55A. An example for one fitted curve is shown in Figure 55B. The exponential decay (t1), that reflected the 
shape of the curve, was then used as coefficient to describe the shapes of the curves and plotted for wild type and dia embryos (Figure 55C). The mean exponential decay of Cpa enrichment at the actin cap in wild type embryos was around 0.5 compared to around 1.3 in dia. This reflected the flatter shaped curve in dia compared to wildtype. Concluding from this data, I could show that Dia is responsible for the organization of the cortical actin network with the plus ends directing to the rims of actin caps.
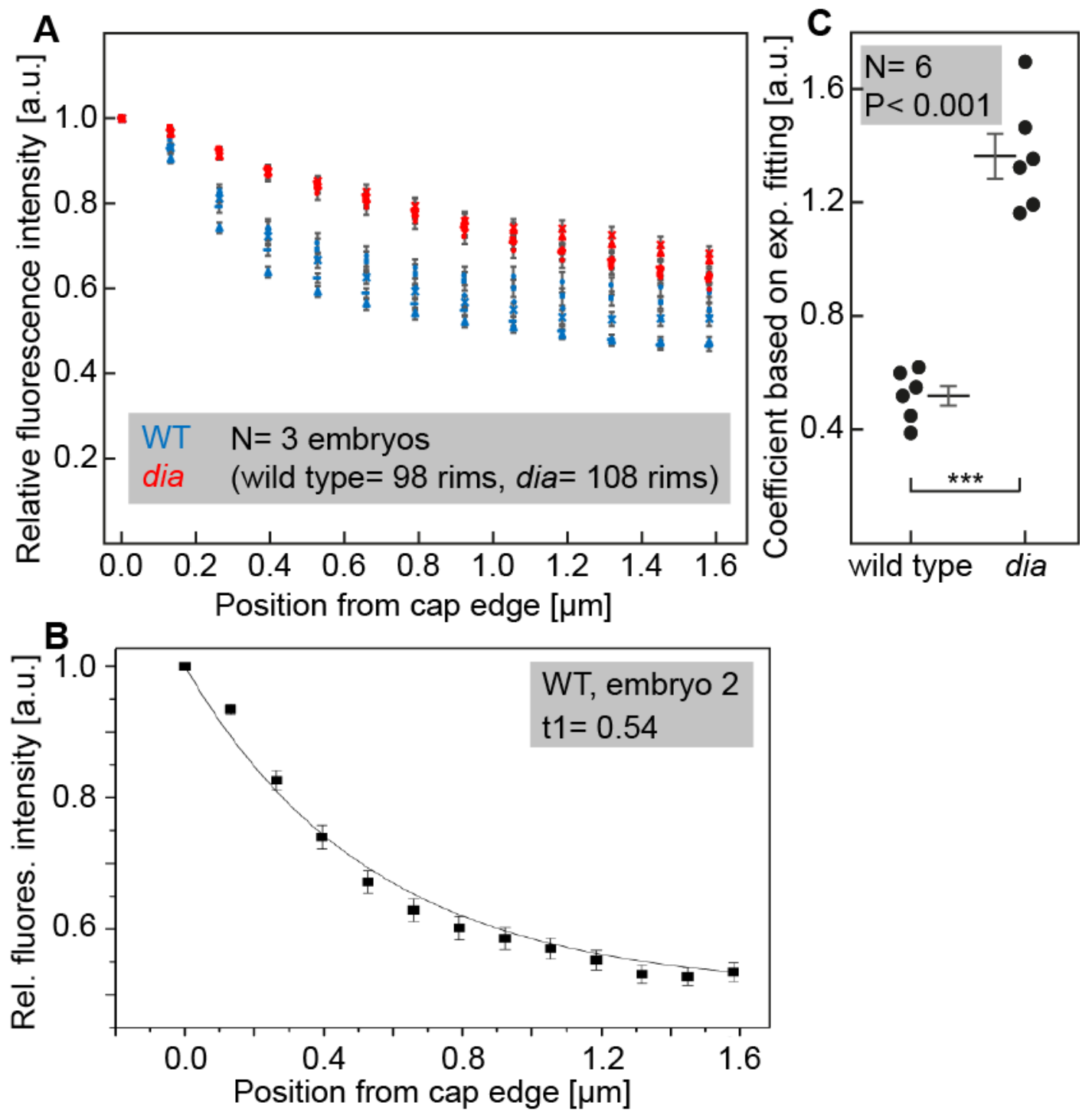

Figure 55 Cpa enrichment at cap rims in wild type and dia.

(A) Relative fluorescence intensities of Cpa staining measured from the edges of actin caps in wild type (blue) and dia (red) embryos. Each data point reflects the averages from one embryo. (B) Example of a fitted exponential curve to the data points from one wild type embryo. $t 1$ is the exponential decay. (C) The coefficient based on the exponential decay plotted for wild type and dia as indicated. Horizontal bars represent the mean and error bars SEM. $\mathrm{P}<0.001$. 


\section{The amount of F-actin does not change in caps of dia embryos}

The stainings of F-actin in wild type and dia and the disorganized F-actin cortex in dia mutants led to the impression, that the amount of cortical F-actin was not significantly different, although Dia is the only formin that is known to play a role during cellularization. To assure, that the disorganization of the actin cortex in dia is not due to a reduced amount of cortical F-actin, I imaged the co-stained wild type and dia embryos with same laser intensities and settings and measured the fluorescence intensity of the signal (Figure 56). In Figure 56A examples of wild type and dia syncytial embryos are depicted. The images showed no visible difference in F-actin intensity. Imaging was done by conducting z-stacks of interphases during syncytial development and then summarized. Four frames of a size 256x256 pixel were measured per embryo for fluorescence intensity, which was plotted for both phenotypes (Figure 56B). In total three embryos per phenotype were measured and the mean value is shown as vertical line in the plots. The embryos were chosen by cap size and nuclear size to measure comparable stages. The measurement showed, that indeed no significant difference in fluorescence intensity of the F-actin signal between wild type and dia could be detected, so the disorganization of the cortical F-actin seemed to be dependent on Dia directly.
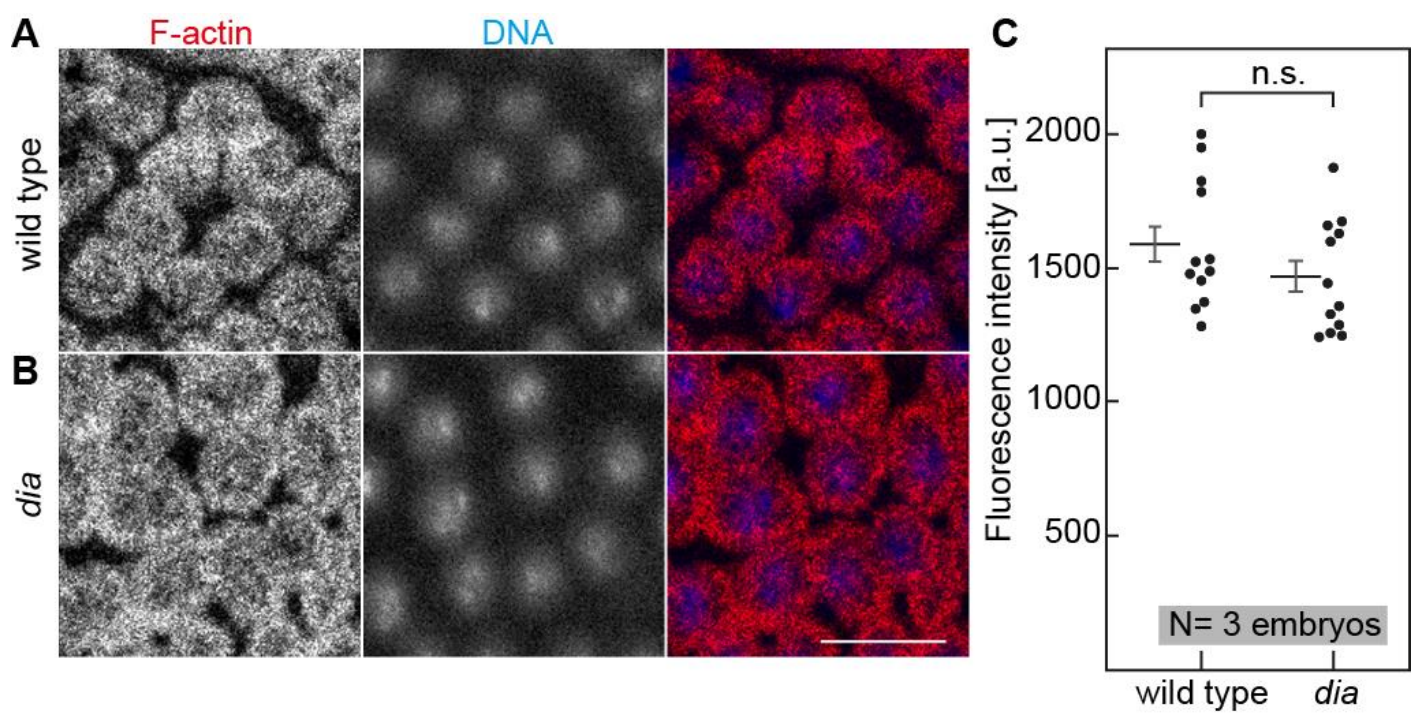

Figure 56 Fluorescence intensity of F-actin in wild type and dia syncytial blastoderm embryos. (A) Wild type and dia embryos were co-stained against F-actin by Phalloidin (grey/ red) and DNA (grey/ blue). Merged images are shown in right panels. The interphase embryos were imaged with same laser intensities and settings and the conducted z-stacks were summarized. (B) Four frames of three embryos per genotype were measured for fluorescence intensities and plotted. The mean is shown by a vertical line and error bars represent SEM. Scale bar $10 \mu \mathrm{m}$. 


\section{Cpa is misorganized in dia embryos during early cellularization}

Next to the localization of Cpa in syncytial blastoderm interphases, I also analyzed the localization of Cpa during early cellularization in interphase 14 . Staining of wild type and dia mutant embryos against Cpa and F-actin by Phalloidin are shown in Figure 57. In early cellularizing wild type embryos, Cpa was still detectable at the rims of the actin caps and was enriched at the basal tips of the invaginating cellularization furrows as shown in the side views in Figure 57A. The top views of wild type embryos show a sharp enrichment of Cpa and F-actin in a hexagonal pattern, marking the invaginating membrane. In cellularizing dia mutant embryos, Cpa and F-actin were detectable at the caps and the cellularization furrow with enrichment at the basal tips. However, also during early cellularization, the enrichment of Cpa at the rims of the actin caps was not visible in the side view (Figure 57B). Cpa and F-actin also marked the invaginating furrows in dia mutant embryos as shown in the top view (Figure 57B), but also here the signal seemed not as sharp as in wild type.

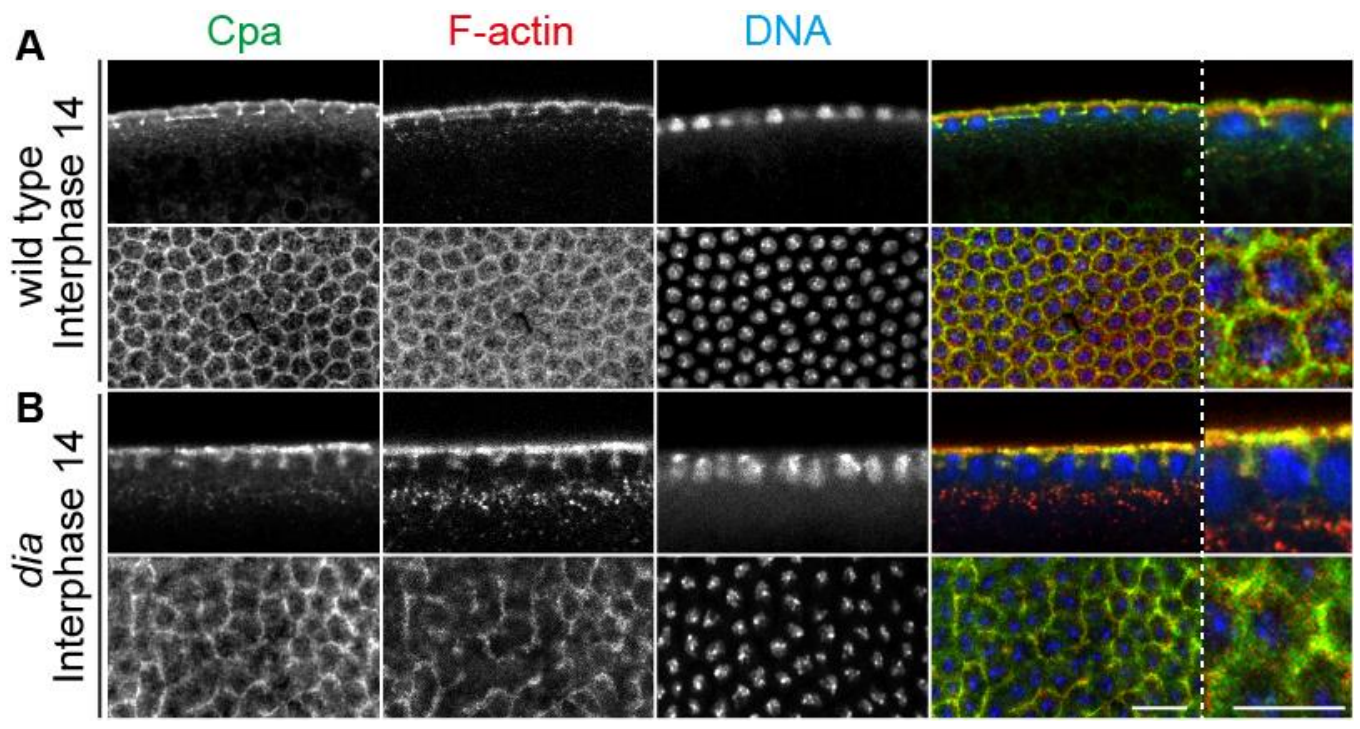

Figure $57 \mathrm{Cpa}$ and F-actin enrichment at actin caps and basal tips in wild type and dia during early cellularization.

Fixed (A) wild type and (B) dia mutant embryos were stained against Cpa (grey/green), Factin by Phalloidin (grey/ red) and DNA (grey/ blue). Merged panels, with a zoom-in, are shown in right panels. Side views of both genotypes are depicted in the upper panels, top views in lower panels. Scale bar $10 \mu \mathrm{m}$. 


\section{Discussion}

\subsection{Introduction}

In part A of my thesis, I could show, that the unconventional GEF ELMOSponge is upstream of the pathway to form the subapical domain. It is likely, that subapically enriched ELMO-Sponge activates Rap1 to localize subapical proteins. Furthermore, I proposed the hypothesis, that ELMO-Sponge relocalize with onset of cellularization and onset of zygotic gene expression from a more disc like pattern to a ring-like pattern that marks the position of the subapical domain.

However, it was still not clear how this relocalization could happen and how the position of the ELMO-Sponge ring could be defined. In the second part of my thesis, I described the function of the formin Dia on the restriction of subapical proteins to their cortical domains and investigated how Dia could affect the organization and polarity of the cortical actin filaments and the function of F-actin polarity on cortical domains.

\subsection{Dia acts indirectly on subapical cues}

In the second part of my thesis, I examined the role of Dia on the formation of the subapical domain. I could show, that Dia influences the subapical restriction of Sponge, Canoe, Bazooka and Armadillo during early and midcellularization as this was affected in dia mutant embryos. As described before, Dia does not only play a role during the formation of the subapical domain but also restricts proteins of the lateral domain from spreading into the basal domain (Yan et al., 2013). However, the role of Dia in separating lateral from basal domain seems to be more indirect. Dia, that is enriched at the basal furrow canal, nucleates and elongates filamentous actin, stabilizing the actin cortex of the basal domain and reducing membrane turnover at this position. In dia mutants, the weakening of the basal cortex could lead to spreading of lateral proteins into the basal domain. Interestingly, basal markers, like Slam, stay restricted to their domain, which could not be explained yet (Yan et al., 2013).

Although Dia has an influence on the subapical enrichment of all tested proteins, their localization in dia mutants was different. Whereas Sponge and Canoe still showed subapical enrichment in dia and leaked into the lateral domain in higher amounts than in wild type, Bazooka and Armadillo completely lost subapical enrichment. Interestingly, both Sponge and Canoe 
are on top of the subapical domain formation pathway directing Bazooka, which itself directs Armadillo, to its subapical position during the course of cellularization (Choi et al., 2013; Schmidt et al., 2018). By this observation, I hypothesize that the formation of the subapical domain does not only depend on Dia. Furthermore, the localization of the marker proteins seems to be refined, possibly within this pathway or yet unknown proteins playing a role on this pathway. A hint for this hypothesis is, that Canoe is not only dependent for proper subapical localization of Bazooka during cellularization but also Bazooka acts on Canoe localization in a reverse loop (Part A, Choi et al., 2013). Possibly, reverse loops could help to insure the correct set up of the subapical domain during cellularization.

Furthermore, one has to keep in mind, that the dia $a^{\text {sy5 }}$ allele is described as hypomorphic. Although I was not able to detect protein by staining or western blot, it is still possible, that there is still some residual amount of functional protein. Although there is a dia allele (dia $\left.{ }^{s y 1}\right)$ available, that has a premature stop codon at position 455 , it is not possible to test the localization of subapical proteins in mutant embryos of these alleles, as female flies carrying the mutation in their germline do not lay eggs. Another possibility to test how the subapical proteins behave, is to use a formin inhibitor (SmiFH2) which it at least described to function on vertebrate formins in cell culture (Isogai et al., 2015). Furthermore, several other proteins belonging to the class of formins are described in Drosophila (reviewed in Bogdan et al., 2013). Although a function for other formins in the process of cellularization is not described, it is still possible that another formin could, at least partially, substitute for Dia function.

\subsection{Less Dia does not lead to less cortical F-actin}

As I could not detect a subapical enrichment of Dia, Rho signaling or F-actin during early or mid-cellularization, I suspected, that Dia does not play a direct role in positioning of subapical marker proteins. That arises the question, how Dia could act on these proteins. As explained before, Canoe and Bazooka bind both to a cortical F-actin scaffold (Choi et al., 2013; Harris and Peifer, 2005) and as Dia can nucleate and elongate F-actin (Yan et al., 2013), I tested how the cortical F-actin network is affected in dia mutants. Surprisingly, I could still detect F-actin along the cellularization furrow, although the cortical F-actin seemed to look more unorganized than in wild type. Furthermore, the 
quantification of F-actin amounts at the actin caps of wild type and dia mutant embryos during syncytial blastoderm interphases revealed, that there is no significant difference. From this data I concluded, that the amount of cortical F-actin is not changed in dia during syncytial blastoderm and cellularization and I hypothesize, that the mislocalization of subapical proteins is not due to a reduced amount of a cortical F-actin scaffold.

Again, the fact that I could not find a difference in F-actin amount in wild type and dia could, at least partially be an effect of the hypomorphic mutation. However, other actin nucleators and elongators could at least partially overtake this function in dia mutant embryos. For syncytial blastoderm interphase caps, it was described before, that the Arp2/3 complex, that can produce branched F-actin, is needed for the formation of functional caps and cortical F-actin (Zallen et al., 2002). In contrast, Dia is needed for the formation of metaphase furrows during syncytial blastoderm, as these are absent also in dia ${ }^{s y 5}$ mutants. However, several formins are described for Drosophila, but either mutants of these formins, like Cappuccino, Frl, Fhod1, did not show phenotypes during cellularization or they were not tested yet.

\subsection{The polarization of cortical F-actin defines the interphase of cap and intercap domains}

I could show, that Dia seems acts on the formation of subapical proteins indirectly and also not by being responsible for the cortical F-actin scaffold to bind the subapical proteins. So, the question remained how Dia could affect the domain formation. Dia is described to bind to the barbed or plus ends of F-actin filaments to elongate it (Higashida et al., 2004) and I could show that Dia is enriched at the rims of actin caps during syncytial blastoderm interphases. From this data I hypothesized, that the cortical actin filaments of the caps could show a polarized distribution with plus ends enriched at the edge of the caps. This hypothesis was supported by staining against Capping protein alpha $(\mathrm{Cpa})$, that is also a plus end binding protein. The capping protein heterodimer, that consists of alpha and beta subunit was described before as F-actin regulator and tumor suppressor module in human and Drosophila. The regulation of Cpa in vivo was described in Drosophila wing disc cells (Amândio et al., 2014). The authors of this publication could show, that $\mathrm{Cpa}$ and $\mathrm{Cpb}$ are enriched at adherens junctions in the wing disc epithelium. Overexpression of both proteins led to a decrease in apical F-actin levels and 
a reduction in cell size, whereas expression of mutated versions that have a dominant negative effect resulted in increased F-actin levels and tissue overgrowth. During ventral furrow formation in gastrulation, the polarized apical F-actin network is required for apical constriction of epithelial cells (Coravos and Martin, 2016; Lv and Großhans, 2016). The F-actin network with the plus ends enriched at the peripheral adherens junctions provides the basis for MyoII that moves towards the plus ends of actin filaments, generating the force that is needed for apical constriction. As these studies provide evidence for a polarized cortical F-actin network in polarized epithelial cells, I tested the hypothesis that this is also true during syncytial blastoderm development. By staining against Cpa I could not only show that F-actin plus ends were enriched at the edges of actin caps but also that this was dependent on Dia. The sharp line of Cpa surrounding the actin caps was broadened in dia mutants. Furthermore, at onset of cellularization, I could detect Cpa enriched along the whole actin cap and not at the edges as it was in wild type. These observations led to the hypothesis, that Dia is responsible for the organization of cortical F-actin, with plus ends being enriched at the edges of the interphase caps and also organizing the cortex of the cellularization furrow.

The fact that cap domains and the cellularization furrow show a polarization of their cortical F-actin that is dependent on Dia leads to the question how this polarization could affect the formation of new cortical domains. I hypothesize that the polarization with the F-actin plus ends at the rim of the cap domain could help to define the interface of two domains (Figure 30). In the case of actin caps the interface of cap and intercap domain. By this definition of the interface, the position of the newly inserted subapical domain during onset of cellularization could be determined.

Following this hypothesis, the question how this could be done mechanistically arises. Firstly, the polarized actin cytoskeleton could lead to a polarized transport of subapical proteins to the area of the newly formed domain. Secondly, the polarization of the cortical F-actin could somehow lead to a spatial restriction of proteins bound to this scaffold (Figure 58). Thirdly, it is possible, that the polarized cortical actin network is involved in MyoII flows especially during onset of cellularization. Cortical flows of MyoII are known to play a crucial role in various processes by the generation of local forces for example in apical constriction (Munjal et al., 2015), cytokinesis (DeBiasio et al., 1996) or embryonic polarity (Munro et al., 2004). A recent publication also 
showed cortical MyoII flows with onset of cellularization (He et al., 2016). Here the authors could show, that MyoII is recruited by the yet uncharacterized protein Dunk. Possibly, the cortical MyoII flow is supported by the polarized actin cytoskeleton at the onset of cellularization. To test this hypothesis MyoII flows in wild type versus dia mutant embryos could be analyzed.

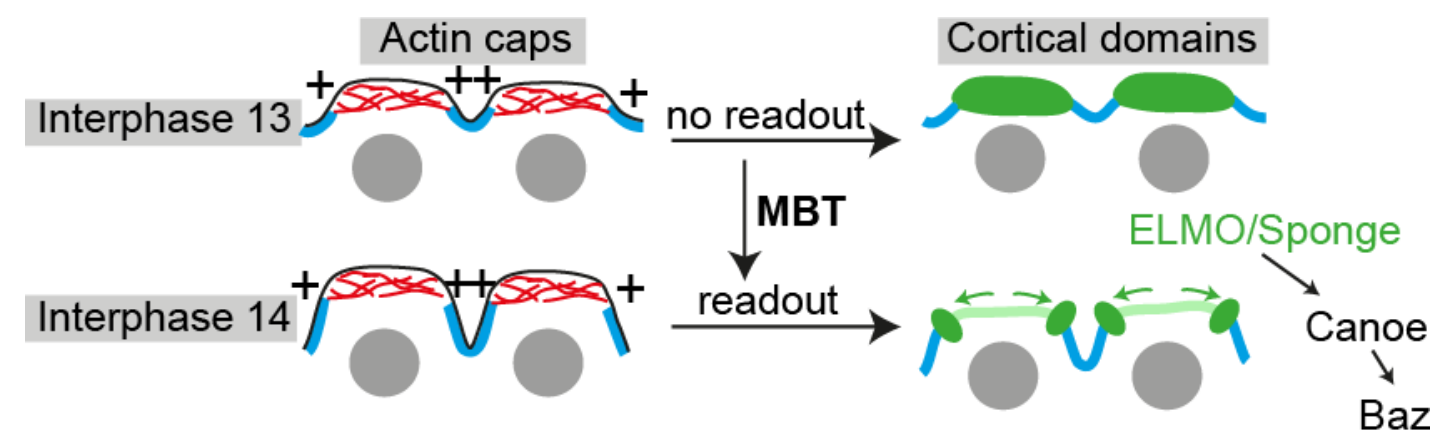

Figure 58 Influence of a polarized actin cortex on the formation of a cortical domain.

Before MBT the actin cortex of actin caps is already polarized but ELMO-Sponge still localize in a disc-like fashion. After MBT the polarized actin network leads to redistribution of ELMOSponge to the interface of cap and intercap domains and formation of the subapical domain by activation of the downstream players.

\subsection{Midblastula transition as a switch for introduction of a new domain}

As I could show in part A of my thesis and discussed before, the switch from maternal to zygotic gene expression by midblastula transition is responsible for the switch in ELMO-Sponge localization from a disc- to a ring-like distribution. For the polarization of the actin cytoskeleton I detected a ringlike enrichment of plus ends at the rims of the cap domain throughout the whole syncytial blastoderm interphases. Concluding, that the polarized cortical actin does not need the input of a zygotically expressed protein. This observation strengthens the hypothesis, that the zygotic expression of a "master regulator" is responsible for the localization change of ELMO-Sponge and the formation of the subapical domain. Taken together, I hypothesize that the polarized cortical F-actin scaffold provides a prepattern for the definition of the interface between cap and intercap domain that is read out by a zygotically expressed, yet unknown, master regulator. This master regulator could then directly or indirectly regulate the change in localization of ELMOSponge during onset of cellularization and with this control the spatial information for the direction of subapical proteins. It would be not surprising if this master regulator would be a transport protein, that moves along actin 
filaments or along microtubules that could also be polarized similar to the cortical F-actin. 


\section{Part C \\ Role of FMNL during early Drosophila embryogenesis}




\section{Abstract}

The role of Formin-like protein (FMNL) on the formation of adherens junctions and actin dynamics in vertebrates makes its Drosophila homolog a good candidate for playing a role during the process of cellularization. The protein belongs to the Dia-family and the vertebrate version is known to nucleate and elongate F-actin. The fact, that FMNL was found in a Yeast-two-hybrid screen as a possible interactor of Slam, that is needed for the invagination of cellularization furrows, made it a good candidate to investigate if there is a function during cellularization. For this analysis a mutant allele of FMNL should be generated. However, I could not find a phenotype. Though I found that $\mathrm{P} /$ Def flies that resulted from a cross of flies with an introduced P-element into the genetic region of FMNL to flies with a large deficiency spanning over the genetic region of FMNL (P/Def flies) expressed less amounts of FMNL by real time quantitative PCR. P/Def flies were viable and fertile but showed reduced fitness and could not be kept as a stock. Embryos from these flies showed a reduced viability in embryonic to pupal stages. To analyze the localization of FMNL and to do further biochemical analysis, I purified an antibody generated against the N-terminus of FMNL. However, stainings with antibody sera and western blot analysis with sera and purified antibodies did not reveal a specific signal. I was successful in the generation of a transgenic fly line expressing FMNL tagged to a GFP driven by its own promotor. Imaging of embryos, as well as in situ hybridization of wild type embryos revealed the expression of mRNA and protein from early embryogenesis on. Also, the dissection of larval and adult tissues revealed FMNL-GFP expression. Strong expression of FMNL-GFP in the larval brain was further analyzed and could be detected in cells expressing a glial cell marker, giving rise to the hypothesis, that FMNL could play a role in the development of the central nervous system. 


\section{Introduction}

\subsection{Proteins of the formin family in Drosophila}

Proteins that belong to the formin-family are known to nucleate and/ or elongate filamentous actin. They are defined by a unique and highly conserved C-terminal formin homology 2 (FH2) domain that mediates the effects on actin (Wallar and Alberts, 2003). The Drosophila genome contains six genes encoding proteins with FH2 domains. Next to Diaphanous (Dia) there are Cappucino (Capu), DAAM, Formin 3, Fhos and FMNL (Frl in flybase).

The formin which is best researched in Drosophila is Dia. Among functions during various cytokinetic events like spermatogenesis, follicle cell formation or neuroblast division, Dia also contributes to early embryogenesis (Part B,Afshar et al., 2000; Castrillon and Wasserman, 1994). Embryos lacking maternal Dia have perturbed metaphase furrow formation, lack pole cells and have defects during gastrulation (Afshar et al., 2000). Capu and Fhos do not show phenotypes during early cellularization. They have functions in oogenesis and tracheal development (Emmons et al., 1995; Lammel et al., 2014). Also for DAAM and Formin 3 no functions in early embryonal development are described, they have functions in tracheal development and muscle development (Matusek et al., 2006; Molnár et al., 2014; Tanaka et al., 2004).

For FMNL, which potential function for cellularization should be investigated during this study, there is also no function during early embryogenesis described, yet. However, Dollar et al. (2016) could show, that FMNL functions in planar cell polarity during eye development and, together with DAAM, in axon growth in the mushroom body of the brain. In flies lacking FMNL or overexpressing dominat negative FMNL the omnatidial rotation is affected. Dollar et al. (2016) could also show, that Drosophila FMNL is autoinhibited and activated by Cdc42, like its mammalian homologe.

\subsection{Formin-like (FMNL) is the sole homolog in Drosophila}

In vertebrates Formin-like Proteins (FMNL) build a group of three members, whereas only one FMNL exists in Drosophila.

The function of vertebrate FMNL was researched in different cell culture models. In human breast cancer cell lines in 3D culture, FMNL2 is required for the formation of adherens junctions downstream of Rac1 (Grikscheit et al., 
2015). The authors could show, that FMNL2 is not only needed for actin assembly and turnover at the adherens junctions, but also that FMNL2 can be recruited to the plasma membrane by Rac1 activity. Another function that was shown in this publication is that FMNL2 is also involved in lumen formation of these cell epithelia. Together the data from this study indicate a function for FMNL2 in the formation of human epithelia.

Another role for mammalian FMNL2 was described in mouse B16-F1 melanoma cell culture (Block et al., 2012). These cells are motile and built large lamellipodia and filopodia especially when plated on fibronectin or laminin coated surfaces (Xue et al., 2010). In these cells FMNL2 was not only expressed endogenously but also had a function in the formation of lamellipodia as the protrusion rate of lamellipodia was reduced by knock down of FMNL2 (Block et al., 2012). With this also the migration rate of the cells was reduced. Another study in this cell culture system revealed that FMNL2 regulates vesicle trafficking and cell polarity downstream of Cdc42 (Kage et al., 2017a) and it has been shown that it elongates F-actin in vitro (Block et al., 2012). In contrast to the work in breast cancer cells described before, an activation by Rac1 could not be detected (Block et al., 2012). Instead, Block et al. (2012) describe the activation of FMNL2 downstream of Cdc42. This is consistent with the study of Dollar et al. (2016) in which an activation by Cdc42 could also be shown for Drosophila FMNL. Dollar et al. (2016) described a role for FMNL in establishment of polarized planar polarity during ommatidial rotation in eye development of Drosophila. Other studies provide a role for mammalian FMNL in colorectal cancer (Li et al., 2010).

The domain structure of FMNL2, which also resembles the domain prediction of Drosophila FMNL, is related to Dia, another member of the formin-protein family. Figure 59 shows the domain structure of FMNL (reviewed in Kühn and Geyer, 2014). The N-terminal regions contain the Diaphanous inhibitory domain (DID) which is necessary for autoinhibition of the protein by binding to the C-terminal Diaphanous autoregulatory domain (DAD). This autoinhibition can be released by binding of small GTPases to the N-terminal GTPase-binding domain (GBD). C-terminal to the DID domain a dimerization domain (DD) which is necessary for the formation of dimers in the active form of the protein is followed by a coiled-coil domain (CC). The formin homology 1 and 2 domains $(\mathrm{FH} 1,2)$ which comprise the C-terminal half of the proteins, are needed for the nucleation and elongation of F-actin. The FH1 domain binds 
the profilin-G-actin-complex whereas the FH2 domain incorporates the Gactin into the F-actin filament. Formins of the Diaphanous family do not only show a similar domain structure but also related regulation (reviewed in Kühn and Geyer, 2014). In the autoinhibited state, the protein forms a ring-like structure, which is resolved by binding of a small GTPase. For FMNL it is known, that the small GTPase Cdc42 can activate the protein, whereas Dia is activated by Rho GTPases. Furthermore, another co-factor can be necessary to fully activate the formin.

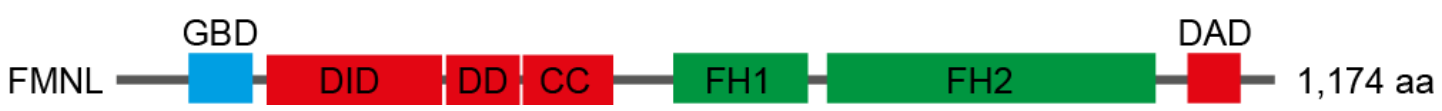

Figure 59 Domain structure of FMNL.

Shown is the known domain structure of Drosophila FMNL. (GBD) GTPase-binding domain, Diaphanous inhibitory domain (DID), dimerization domain (DD), coiled-coil domain (CC), Formin homology domain 1, 2 (FH1, FH2), Diaphanous autoregulatory domain (DAD).

\subsection{Aim of this study}

In this study, the function of FMNL during early embryogenesis should be investigated. This should be done by the making of a mutant by imprecise excition of a P-Element or CRIPSR mutagenesis. Furthermore, the expression and localization of RNA and protein should be analyzed in early embryos by western blot, in situ hybridization, antibody staining and the generation of a GFP-tagged transgene expressed by its own promoter. 


\section{Results}

\subsection{FMNL was found to be an interactor of Slam by Yeast-Two- Hybrid-Screening}

The formin FMNL (CG32138) was found to be a possible interactor with Slam in a Yeast-Two-Hybrid-Screen (Hybrigenics services; Dr. Philip Laupsien, $\mathrm{PhD}$ dissertation) and therefore may play a role for cellularization. A detailed analysis could demonstrate, that the FH2 domain of FMNL and a C-terminal Slam-fragment show a weak interaction whereas an interaction with fulllength Slam could not be detected (Dr. Philip Laupsien, PhD dissertation).

The role of FMNL for cellularization, gene expression and protein localization should be characterized during this study. However, during this work, the generation of a null mutant by imprecise excision of a P-element or CRISPR mutation did not lead to a phenotype.

\subsection{Embryos from P/Def flies show reduced amount of FMNL- RNA and reduced viability}

\section{P/Def flies are viable and fertile}

As the generation of a mutant did not lead to a phenotpye, a deficiency line with full deletion of the FMNL gene (Figure 60) and a fly line with a P-element inserted into the 5' UTR of FMNL (Figure 61) were used for further analysis.

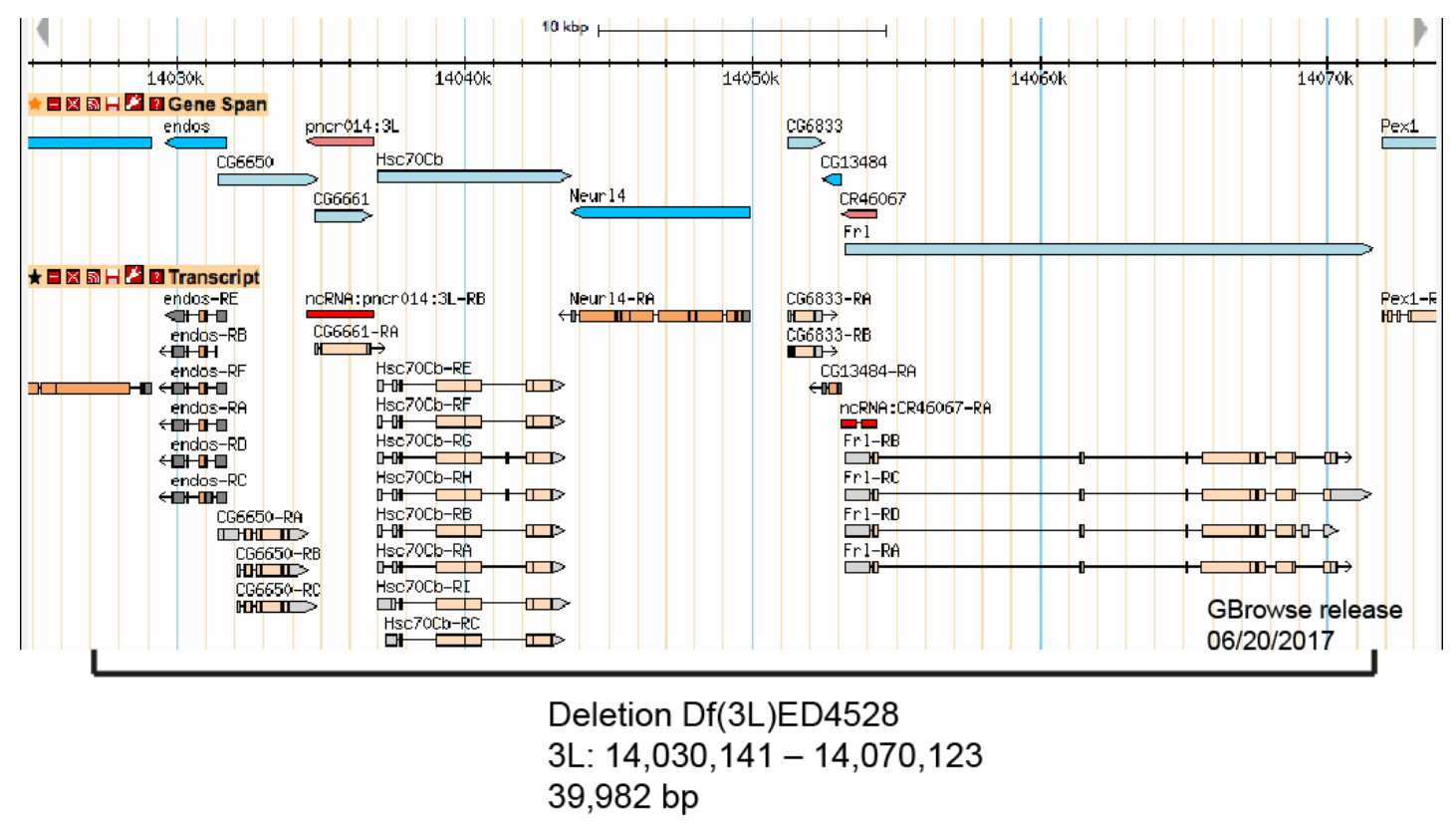

Figure 60 Genetic map of FMNL-deficiency.

Genetic locus of FMNL (frl). The deletion of the used deficiency line Df(3L)ED4528 is indicated with a black line. Taken from flybase.org. 
Both lines are homozygous lethal, however, the P-Element line carries a second lethal, as it was homozygous viable and fertile after cleaning of the chromosome by recombination. However, it was not possible to hold homozygous flies as a stock. Also, the deficiency line was homozygous lethal, as other essential genes were also deleted. Crossing both together, resulted in flies carrying one allele of the P-element insertion and one allele deficient for 3L:14,030,141-14,070,123, from now on named as P/Def flies. These flies were viable and fertile but could also not be kept as a stock.

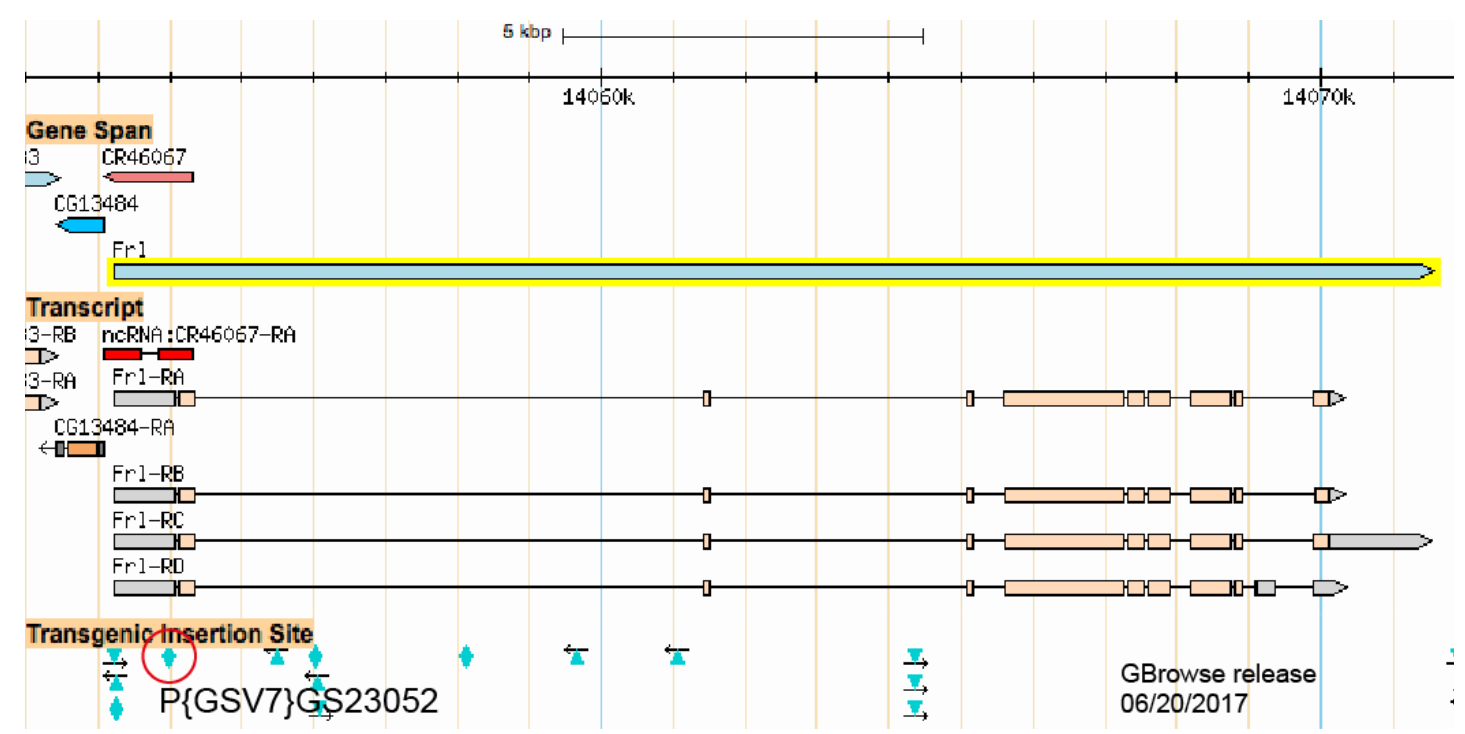

Figure 61 Genetic map of P-Element insertion into the FMNL locus.

Genetic locus of FMNL (frl) is shown as well as it's transcripts. The transgenic insertion site of P\{GSV7\}GS23052 is marked by a red circle. Taken from flybase.org.

\section{Embryos from P/Def flies express less FMNL}

To analyze the expression of FMNL in P/Def embryos, a quantitative qPCR was done with wild type and embryos from P/Def flies. For further analysis it has to be considered, that embryos from crosses of P/Def females with P/Def males can be P/Def (1/3), P/P (1/3) or Def/Def (1/3). Figure 62 shows the PCR cycle number of amplified cDNA, that was needed to reach a critical threshold measured by fluorescence intensity of the incorporated fluorophore for FMNL and actin, a housekeeping gene that was used as reference. The cDNA was prepared by reverse transcription after RNA extraction from embryonic lysates from overnight embryo collections. The cycling number was in logarithmic scale and inversely proportional to the quantity of cDNA, that was reversely transcribed from RNA extracted from embryonic lysates. The negative control $\mathrm{H}_{2} \mathrm{O}$ without cDNA gives high cycling numbers of $\sim 34$ for FMNL and $\sim 35$ for actin, this is also the case for the RNA controls, in which 
wild type or P/Def RNA was added to control for DNA contamination of the RNA extraction. As the cycling numbers for actin with 15.7 for wild type and 15.2 for $\mathrm{p} /$ Def embryos respectively, were nearly the same, the cycle numbers for FMNL for wild type and P/Def embryos could directly be compared. In wild type 22.8 cycles were needed to reach the threshold for FMNL, as in P/Def the cycling number was 25.6, showing that embryos from P/Def flies express $2^{3}$ times less FMNL-RNA than wild type embryos.

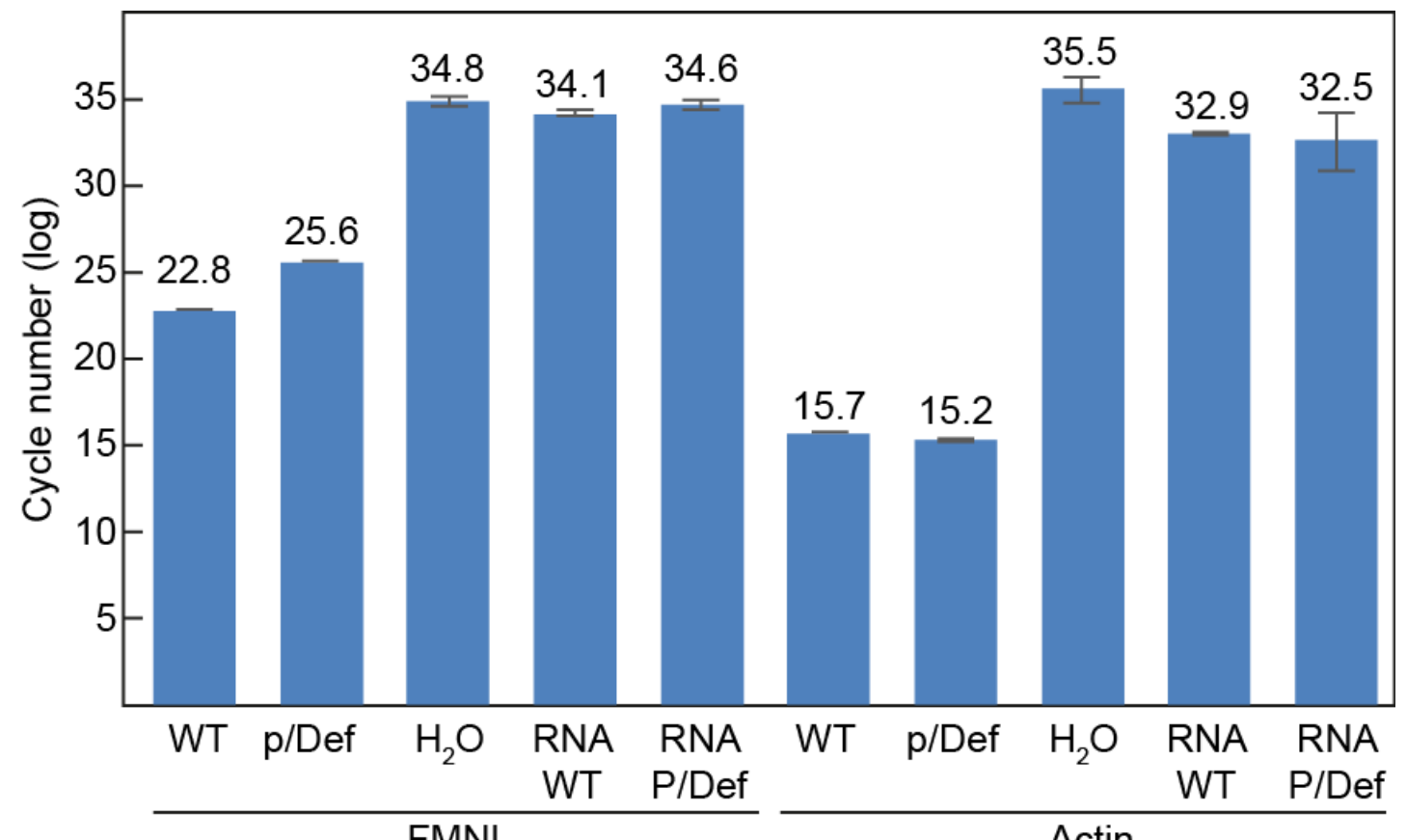

Figure 62 Quantitative real time PCR of p/Def and wild type embryonic extracts.

Cycle numbers (logarithmic scale) are plotted for samples as indicated. FMNL: Primers against FMNL; Actin: Primers against actin (positive control); WT: cDNA sample from wild type embryos; p/Def: cDNA sample from P/Def embryos; $\mathrm{H}_{2} 0$ : negative control with water instead of cDNA; RNA WT: Total RNA extracted from wild type embryos; RNA P/Def: Total RNA extracted from P/Def embryos. Numbers are averaged from two replicates.

\section{Embryos from P/Def flies show a reduced viability}

To analyze the effect of less FMNL-RNA expression in P/Def flies, the viability at different developmental stages was compared with wild type embryos. Again, embryos from wild type and from crosses of P/Def females and P/Def males were used for the analysis and hatched larva, pupa and adults were counted (Figure 63). From 202 wild type embryos 11 embryos died (5.4\%) during development, whereas 81 embryos of 202 embryos from P/Def flies died (40.1\%). Starting with 193 wild type larvae, 9 larvae died before pupation (4.7\%) and 92 larvae of 121 larvae from P/Def flies died (76 \%) and from 194 wild type pupae 2 did not hatch (1.0\%), whereas 8 pupae died of 29 starting 
pupae of embryos from P/Def embryo (27.6 \%) Taken together, embryos, larva and pupa from crosses of P/Def females with P/Def males show increased lethality. Also, for this analysis it has to be considered, that embryos of crosses from P/Def females and P/Def males have different allelic combinations as described before and it is not clear if lethality comes from the second lethal of the P-element line, deficiency of essential genes or reduced FMNL expression.

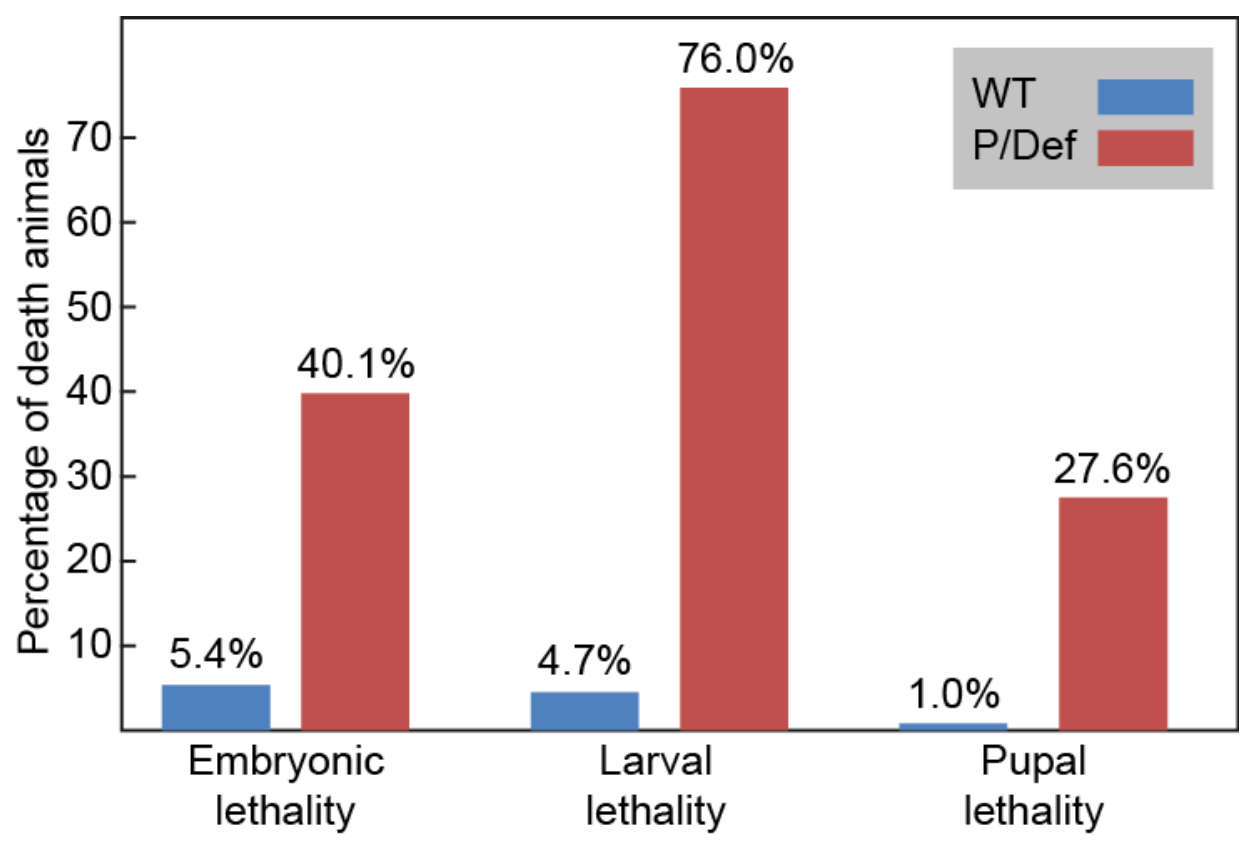

Figure 63 Viability assay of wild type and P/Def flies.

Lethality of wild type (blue) and P/Def (red) during embryonic, larval und pupal development. Lethality rates are shown above the bars.

\section{P/Def flies showed reduced fitness}

Although P/Def flies were viable and fertile, it was not possible to keep them as a stock. To test the general fitness of these flies a negative geotaxis assay was performed. Flies with normal locomotor activity have the tendency to move against gravity. This ability decreases with aging or with reduced locomotor capability (Benzer, 1967). To be able to compare fitness one-day old flies were used for both genotypes and P/TM3 flies were used as control. The assay was performed by transferring single anesthetized flies to plastic tubes with a diameter of around $1 \mathrm{~cm}$ and after waking up, flies were shaken down to the bottom and the distance they crawled up was measured at three timepoints (Figure 64). While P/TM3 flies crawled up to $\sim 15 \mathrm{~cm}$ after 30 seconds, P/Def flies reached only $\sim 1 \mathrm{~cm}$. Also, after 1:30 min, P/Def could only reach $\sim 1.5 \mathrm{~cm}$, indicating that they show less locomotor activity and less fitness. 


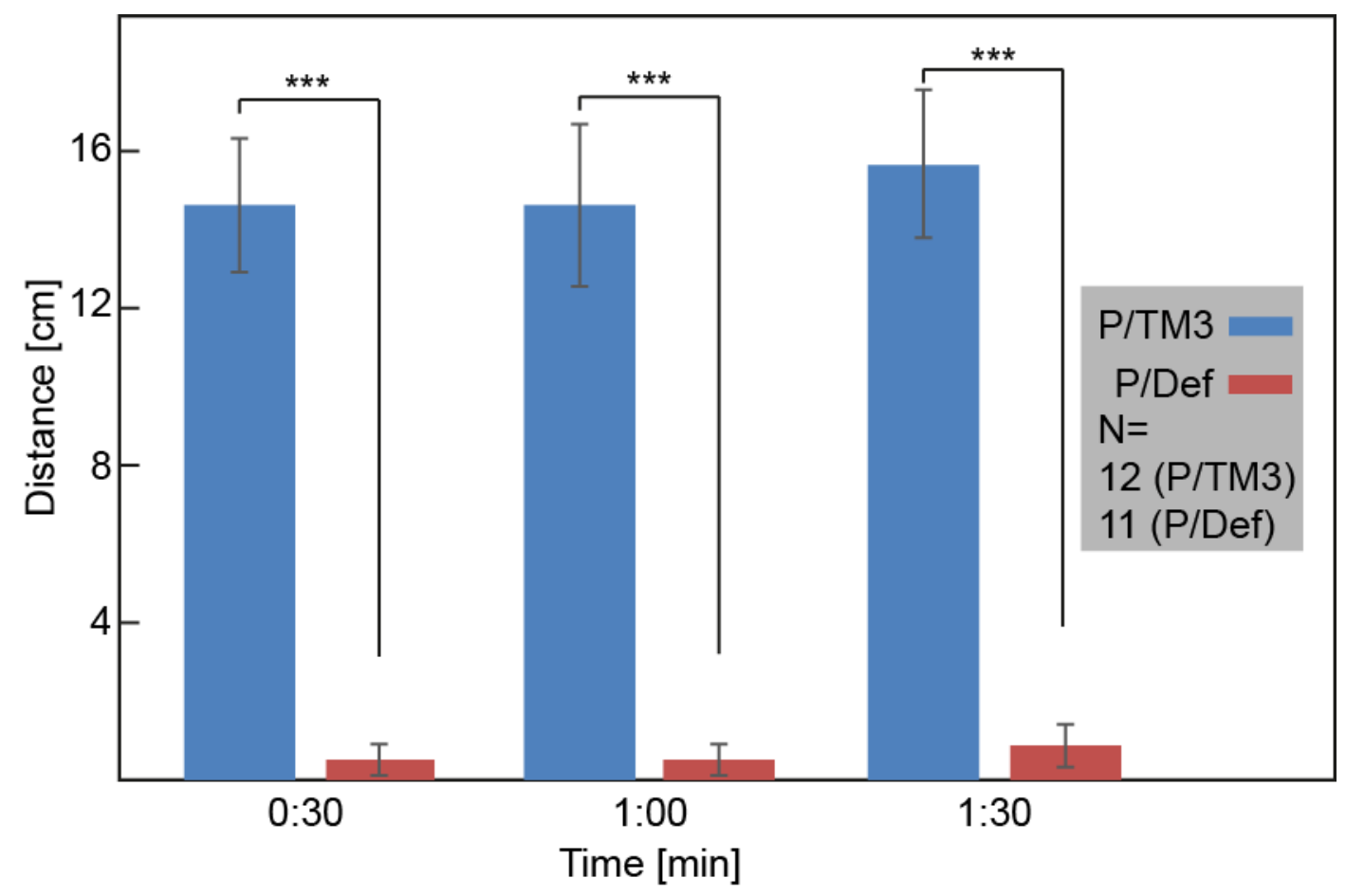

Figure 64 P/Def flies show reduced negative geotaxis.

Averaged distance of P/TM3 (control/blue) and P/Def (red) flies. One-day old adult flies were shaken down in a cylinder and the averaged distance they crawled up is plotted at three different time points indicated. Error bars represent SEM. ${ }^{* * *}: \mathrm{p} \leq 0.001$.

\subsection{The purification of an FMNL-antibody did not lead to a specific signal}

\section{FMNL antibodies did not show specific staining}

Antibodies against the N-terminus of FMNL were raised in rabbit and guinea pig by Henrik Steffen. The antibodies were generated by expression and purification of the N-terminal part $(1-596$ aa) and immunization of rabbits and guinea pigs.

I tested the antibody sera in formaldehyde and heat fixed wild type embryos, however, the fixation procedure did not make a difference. In Figure 65 heat fixed embryos stained with rabbit anti FMNL (Figure 65A) and guinea pig anti FMNL (Figure 65B) are shown, Dlg was used to mark lateral membranes. Rabbit anti FMNL showed partial nuclear enrichment in sagittal views (Figure 65A, upper panel) whereas the top view showed cytoplasmic, possible vesicular, staining with nuclear exclusion (Figure 65A, lower panel). The differences of staining in the different views can be explained by the z-position of the top view that may show a plane were no enrichment can be seen in the channel of the FMNL antibody. Also, in the staining with guinea pig anti FMNL (Figure 65B) nuclear enrichment was shown in sagittal views (Figure 
65B, upper panel) and top views (Figure 65B, lower panel). However, due to the differences in the different views and with different antibodies it is not possible to make a clear statement about FMNL localization by this staining.

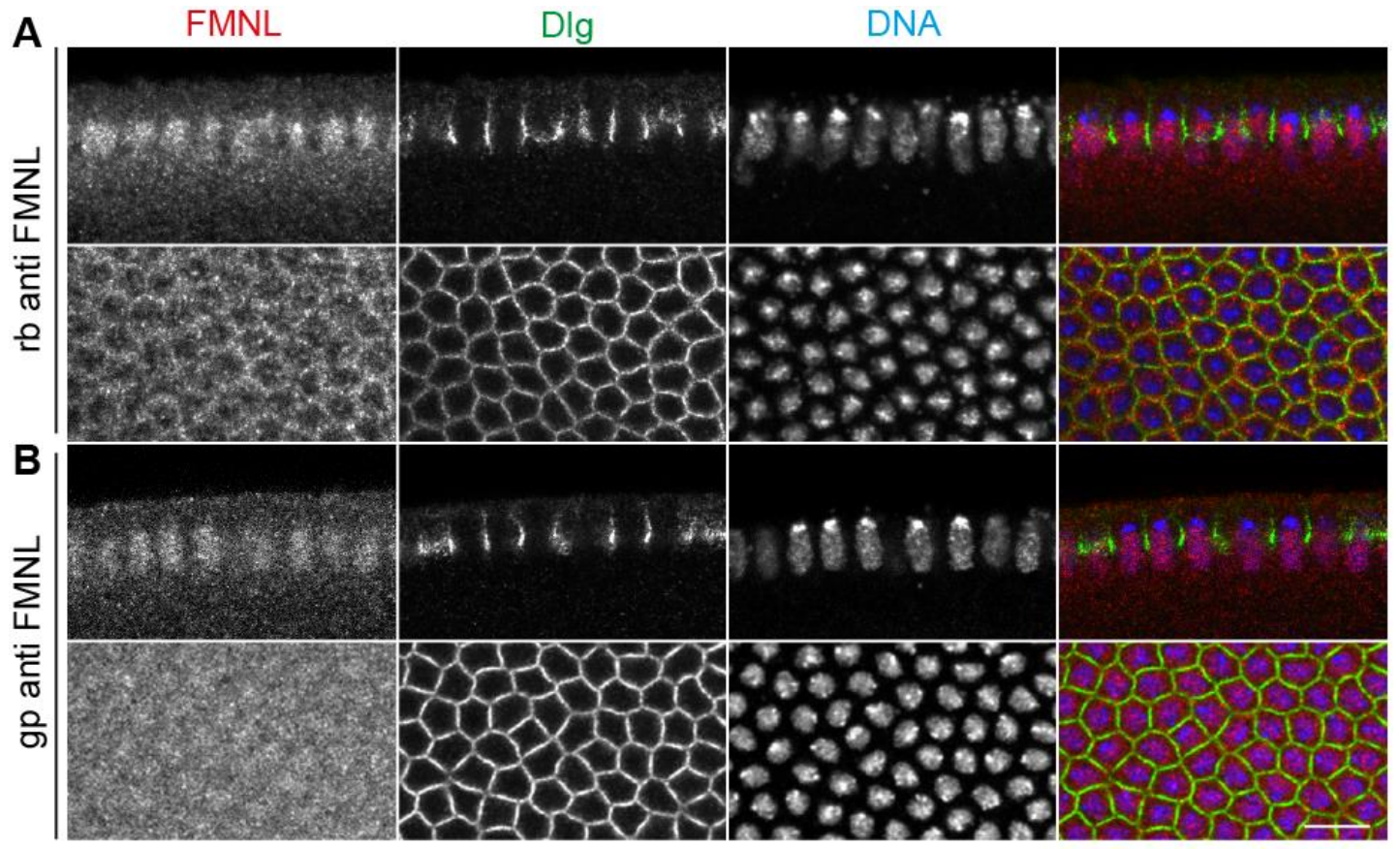

Figure 65 Antibodies against FMNL show no specific signal in wild type embryos.

(A-B) Heat fixed embryos stained for FMNL (grey/red), Dlg (grey/green) and DNA (grey/blue). Merged images are shown in right panel. Upper panels show sagittal views, lower panels top views. (A) Staining with serum of rabbit anti FMNL-antibody, (B) staining with serum of guinea pig anti FMNL-antibody. Scale bar $10 \mu \mathrm{m}$.

\section{Purification of FMNL antibodies did not lead to a specific signal}

As the staining could not show a clear localization, I purified the antibody sera by affinity purification with the protein fragment that was used for immunization. For this procedure, I expressed the FMNL fragment with an Nterminal ZZ-tag and a C-terminal His-Tag in BL21DE cells (Figure 66A). The fragment was running at $\sim 82 \mathrm{kDa}$ and showed a clear induction after it was expressed overnight at $18{ }^{\circ} \mathrm{C}$. After lysis, I purified the fragment with an Äkta over His-Trap columns at $4{ }^{\circ} \mathrm{C}$ as shown in Figure 66B by SDS-PAGE of different fractions and the OD read of A280 during purification in Figure 66C. Although a big amount of the fragment could be found in the insoluble fraction (Figure 66B) it was still possible to purify a suitable amount of the fragment. After the first purification step, the eluted fractions 9-11 were pooled. 
A

ZZ-FMNL2-His in BL21DE $0.2 \mathrm{mM} \mathrm{IPTG}, 18^{\circ} \mathrm{C} ; \mathrm{o} / \mathrm{n}$

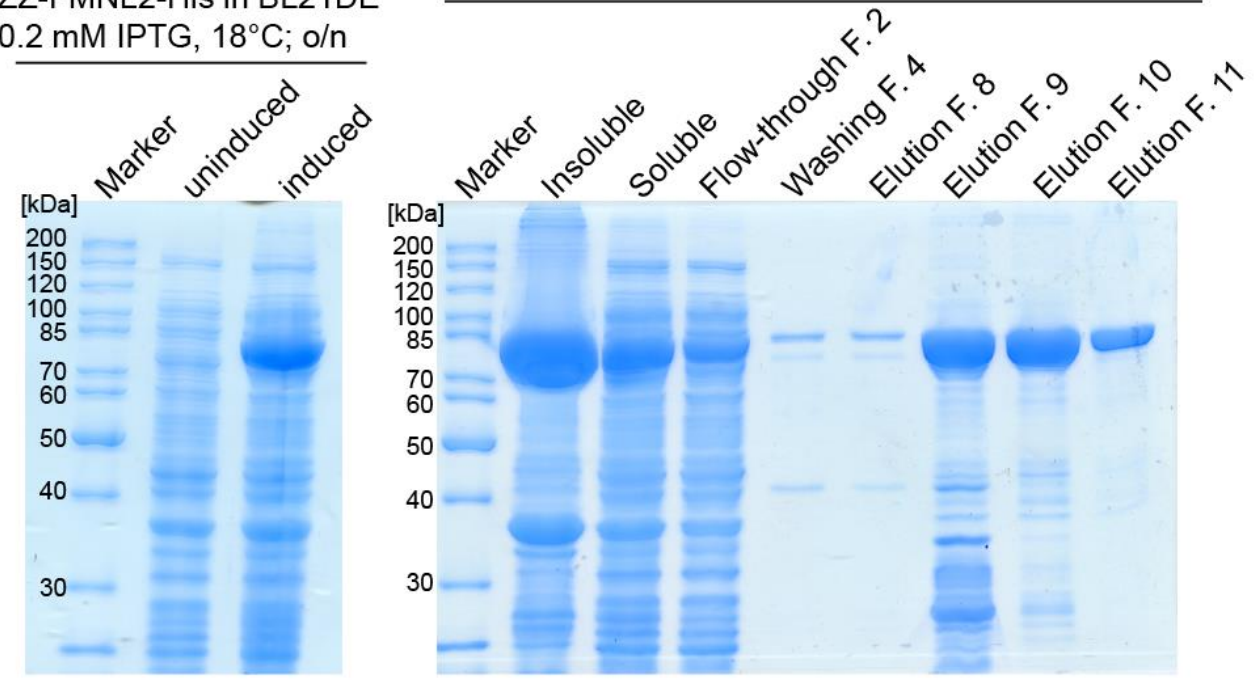

C

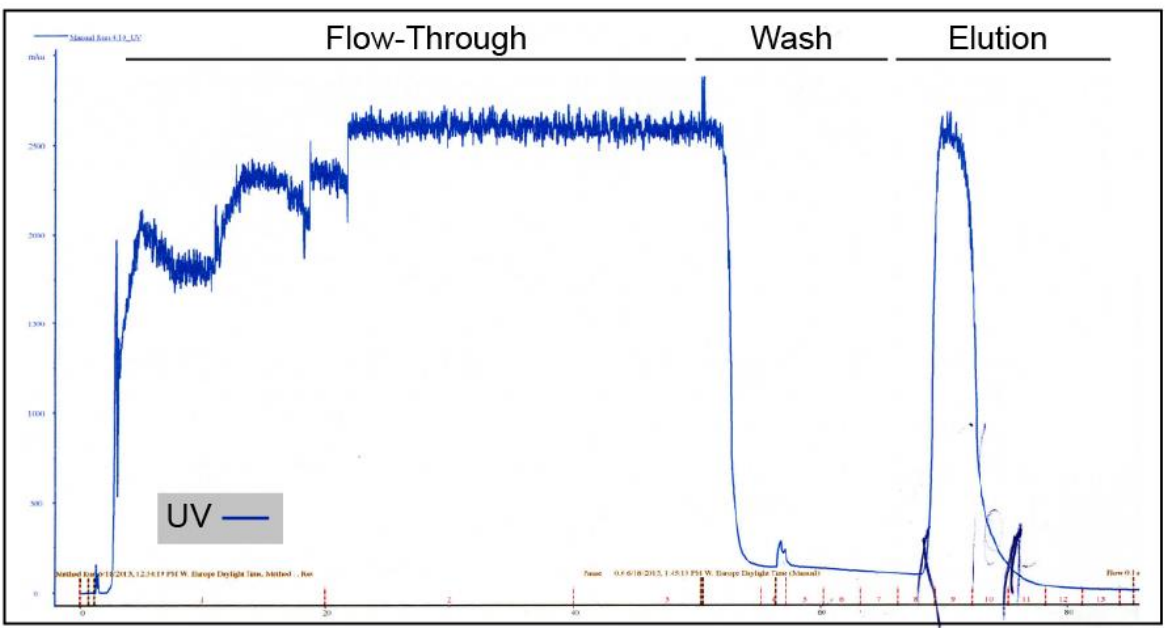

Figure 66 Purification of FMNL-Fragment with N-terminal ZZ- and C-terminal His-tag.

(A) Protein gel of lysed BL21DE bacteria before and after induction of protein expression as indicated. ZZ-FMNL-His Fragment was running at $\sim 75 \mathrm{kDa}$. (B) Protein gel of different fractions as indicated during purification of ZZ-FMNL-GFP with His-Trap columns. (C) Absorbance of $A_{280}$ during purification. Flow-Through, washing and elution steps were as indicated.

As an ZZ-tag can lead to unspecific binding of the antibody, it was removed by making use of a TEV cleavage site between ZZ-tag and the FMNL fragment. Successful cleavage was shown by SDS-PAGE, as the fragment was running at $\sim 70 \mathrm{kDa}$ after cleavage (Figure $67 \mathrm{~A}$ ). The ZZ-tag has a molecular weight of $\sim 15 \mathrm{kDa}$. The second purification was done with His-Trap columns at $4{ }^{\circ} \mathrm{C}$ manually with a pump, however as one of the columns was getting clogged during loading, it was disconnected and eluted manually without washing steps. I loaded the fractions of this column 1 on an SDS-PAGE (Figure 67B). The corresponding OD read of $\mathrm{A}_{280}$ is shown in Figure 67D. As the eluted 
fractions from column 1 were comparable to the elution of the washed column 2 (Figure 67A, B), fractions 2-3 and 5-9 from column 1 and fractions 5-7 from column 2 were used for the coupling to Sepharose and affinity purification of the FMNL antibody from guinea pig and rabbit sera.
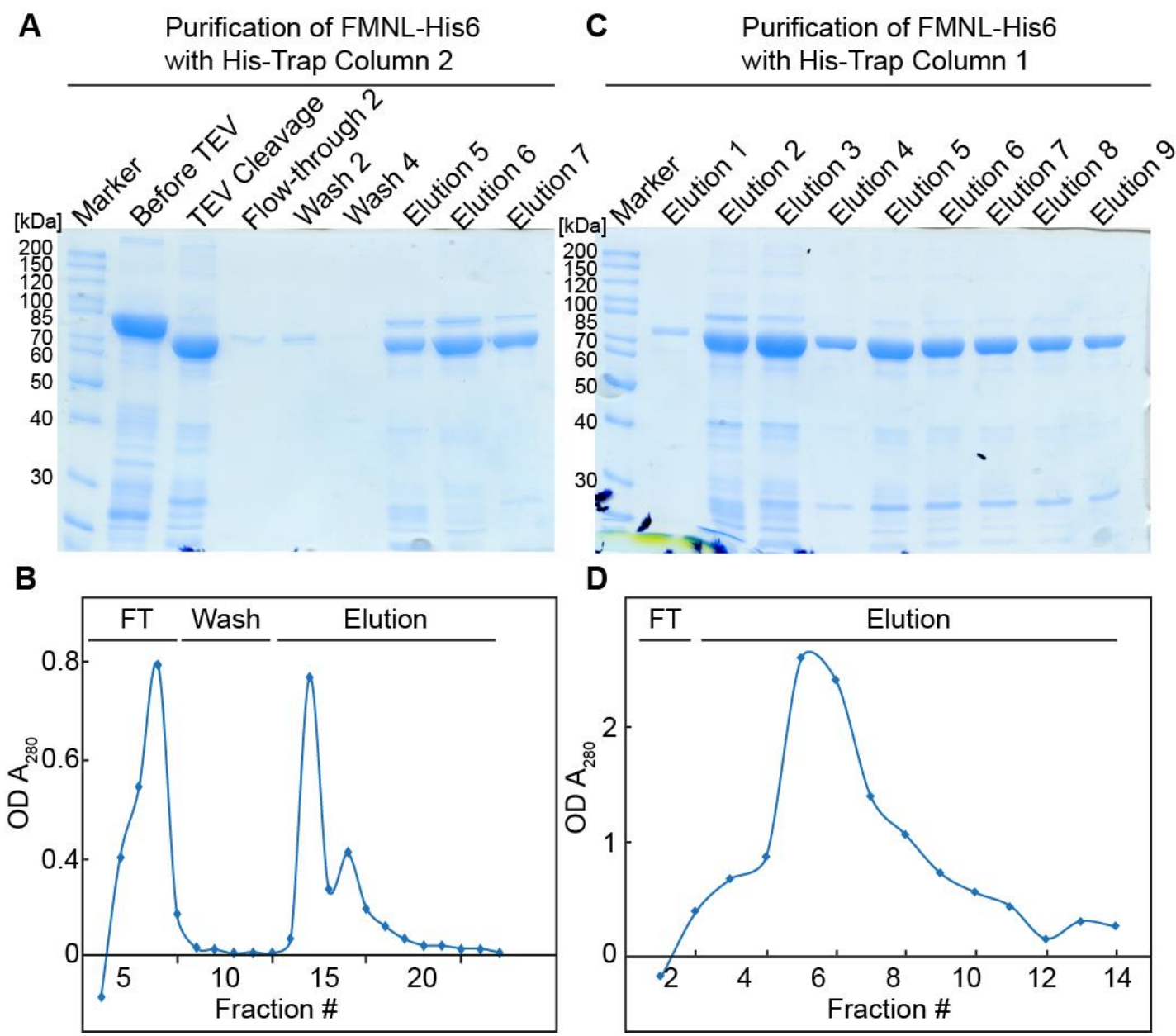

D

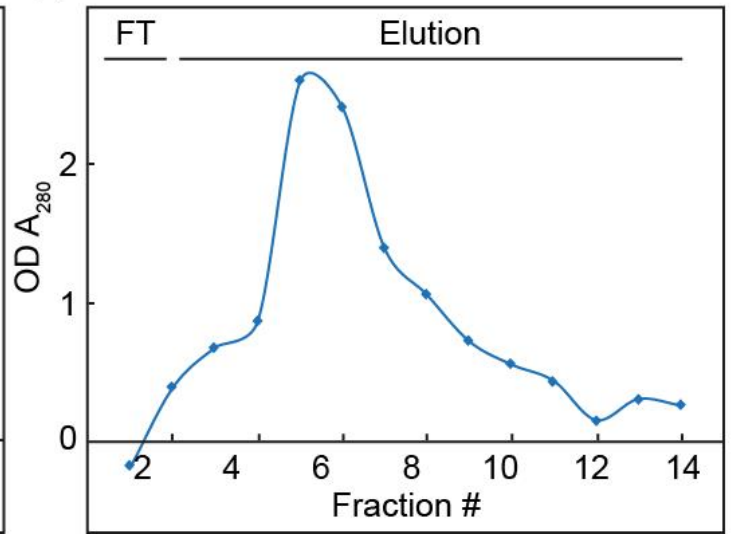

Figure 67 ZZ-Tag cleavage with TEV protease and further purification of FMNL-His fragment.

(A, C) Protein gels loaded with samples during purification with two different His-Trap columns. (A) Samples before and after TEV cleavage are shown in Lane 2 and 3 as indicated. (C) Fractions from elution from column one without washing steps. (B, D) Absorbance of A280 during purification from (B) column 2 and (D) column 1. Flow-through, washing and elution are indicated in graphs.

\section{Purified FMNL antibodies show no specificity in western blot}

Sera and affinity purified antibodies were then tested in western blots of wild type embryo lysates (Figure 68). The full-length FMNL had a calculated molecular weight of $133 \mathrm{kDa}$, but no band could be detected in this range neither with guinea pig serum or purified antibody (Figure 68, left blot) nor with rabbit serum or purified antibody (Figure 68, right blot). Not only, that no band with expected size could be detected, the antibodies, even the purified solutions, showed a lot of unspecific binding. 


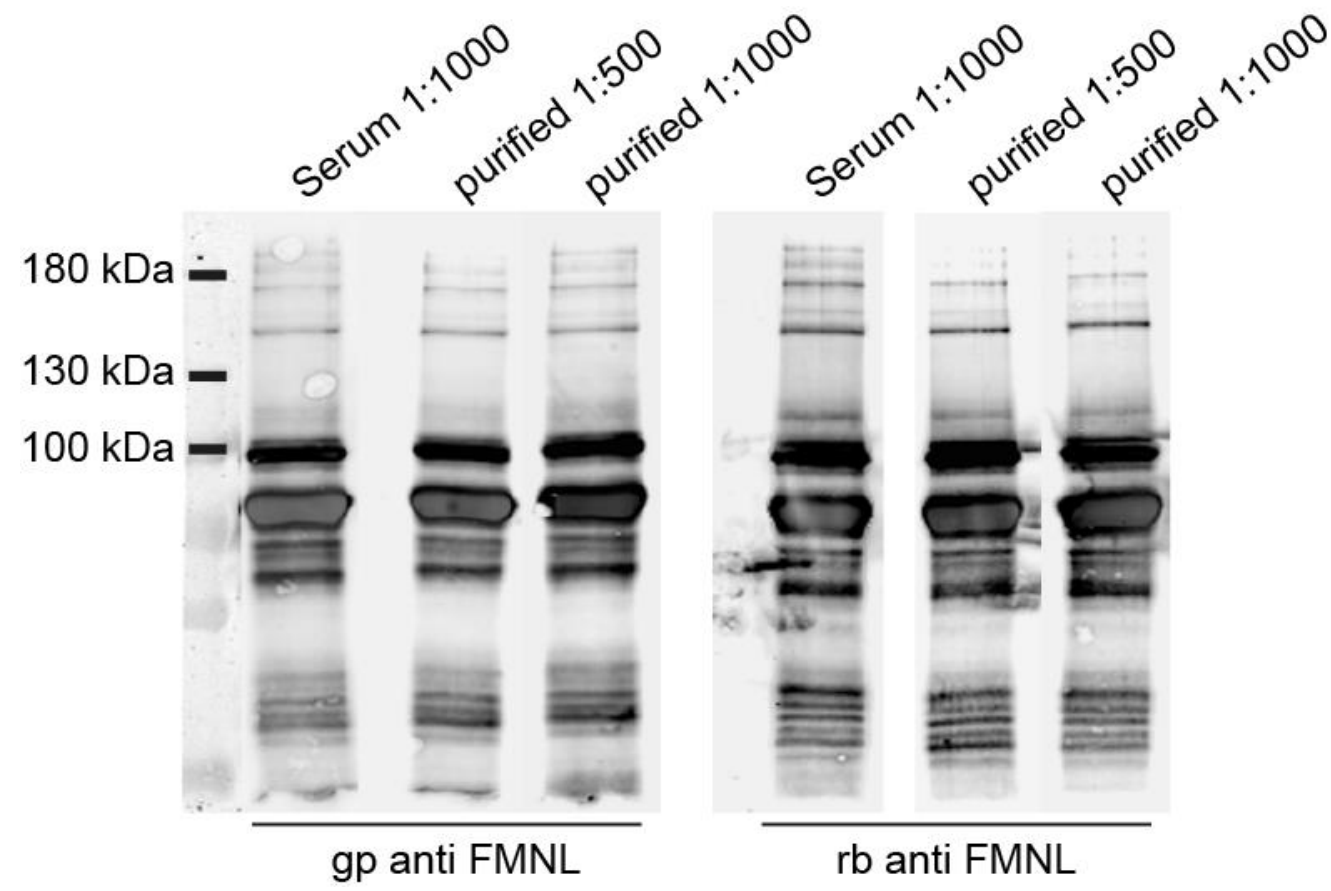

Figure 68 Western blot of wild type embryonic extracts against FMNL.

Western blot of embryonic extracts stained with guinea pig anti FMNL antibody (left panel) and rabbit anti FMNL antibody (right side). 10 embryos per lane were loaded. Staining was performed with unpurified serum and purified antibody solutions as indicated. FMNL should run at $\sim 133 \mathrm{kDa}$.

\subsection{FMNL is expressed during embryonic development}

FMNL mRNA is detected during embryogenesis by in situ hybridization

To show the predicted and by qRT-PCR confirmed embryonal expression of FMNL, I performed a whole mount in situ hybridization.

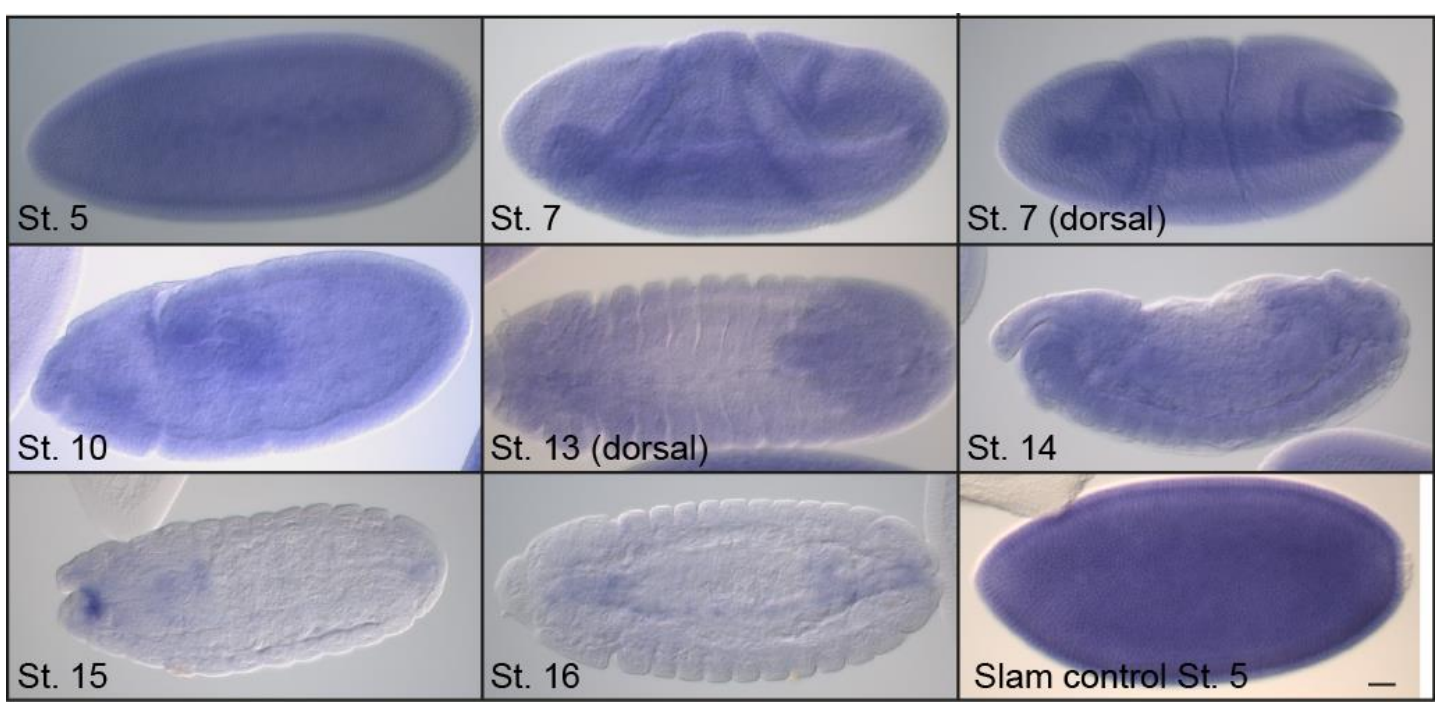

Figure 69 FMNL is expressed during embryonic development.

In situ hybridization of wild type embryos with a probe against FMNL. Stages are as indicated. Image of probe against Slam is shown in lower right panel as control. Anterior is left, posterior is right. Scale bar $10 \mu \mathrm{m}$. 
Probes for FMNL RNA were produced with FMNL cDNA and a probe against Slam was used as control. To get an overview over the expression during embryonal development, embryos were collected overnight. As shown in Figure 69, I found FMNL to be expressed throughout embryonal development from cellularization (Stage 5) on. In stage 15 an enrichment of FMNL could be seen in the anterior part of the embryo. A cellularizing embryo hybridized with a Slam probe was used as a control (Figure 69).

\section{FMNL-GFP is expressed during cellularization}

As the antibodies did not show a specific signal, I generated a GFP-tagged FMNL transgenic fly line. For the transgene a fosmid was used that carried the whole gene region of FMNL with an N-terminal GFP-tag.

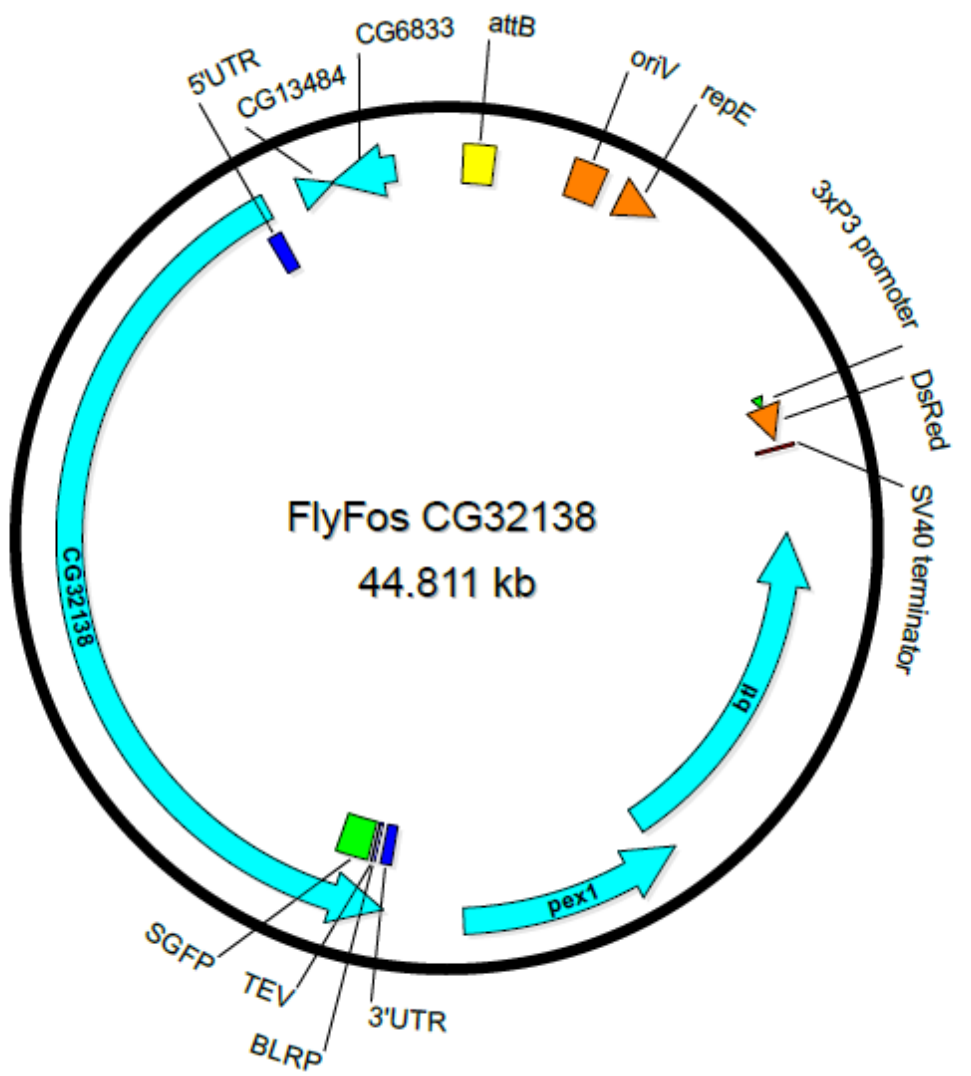

Figure 70 Map of the fosmid used for the generation of FMNL-GFP flies.

The genes integrated into the fosmid are marked in turquois. FMNL (CG32138) is Nterminally tagged with a superfolder GFP (SGFP, green) a TEV site and the target peptide for the birA biotin ligase (BLRP) prior to the endogenic STOP codon. The 5'UTR and 3'UTR of FMNL are marked in blue. The attB insertion site is marked in yellow, the 3xP promoter in green followed by the DsRed sequence (yellow) for detection of successful integration, followed by a SV40 terminator. The origin of replication (oriV) and the F-factor function repE (orange) are for amplification and correct distribution of the fosmid to the daughter cells. 
The construct was inserted into the left arm of the third chromosome at position 65B2 in addition to the endogenous protein. This fosmid was generated by the TransgeneOME project (Ejsmont et al., 2009; transgeneome.mpi-cbg.de) and provided by Dr. Frank Schnorrer. It contained the genetic region of the genes CG6833, CG13484, FMNL (CG32138) with Nterminal GFP, pex1 and btl and a dsRed tag driven by the 3xP3 eye promoter (fosmid map, Figure 70). The inserted construct reflects endogenous expression due to the large genomic region associated with the tagged FMNL region.

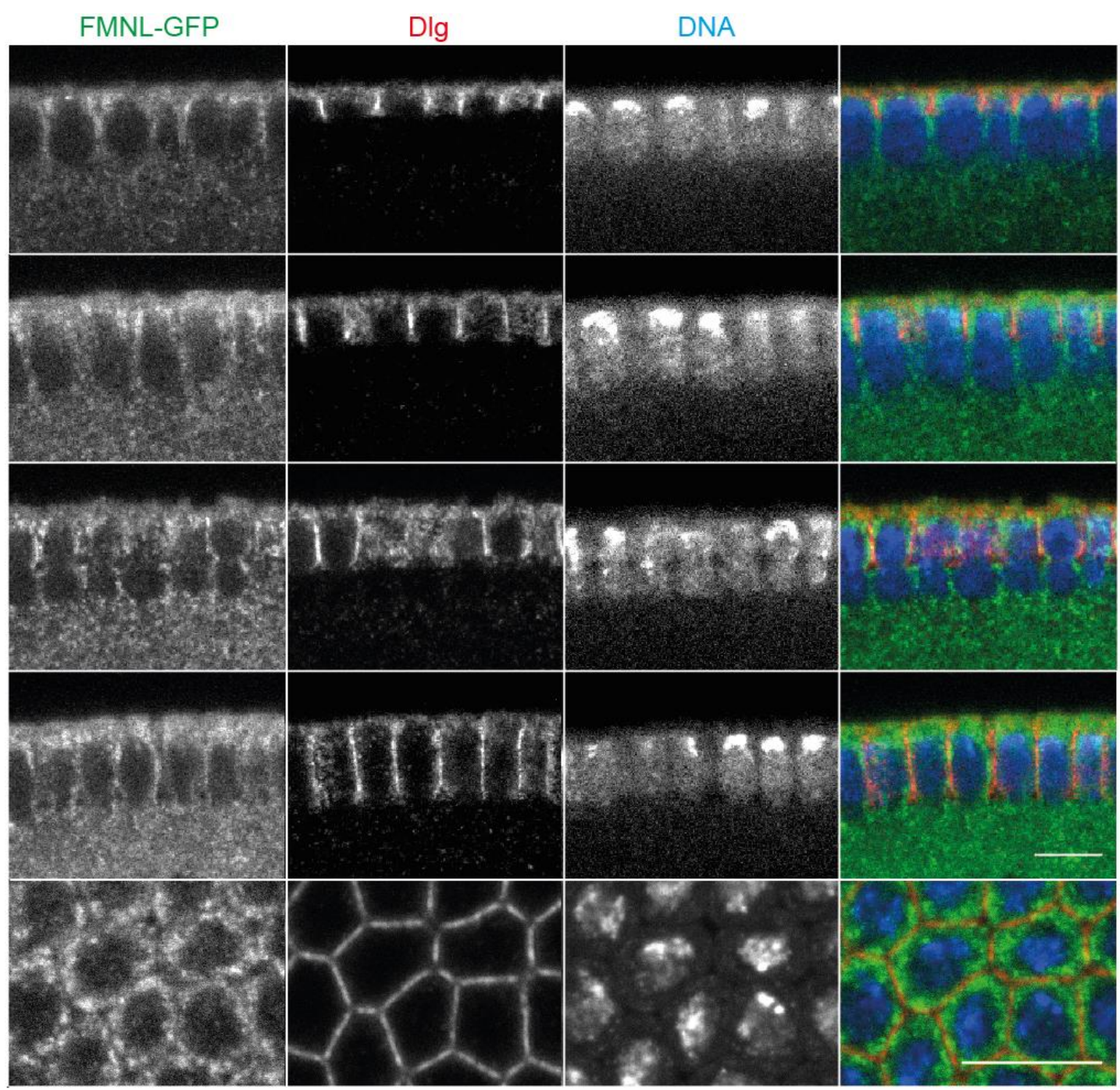

Figure 71 FMNL-GFP is expressed during cellularization.

Images of fixed embryos in early, mid and late cellularization from top to bottom expressing FMNL-GFP stained with GFP-booster (grey/green), Dlg (grey/red) and DNA (grey/blue). Merged images are shown in right panel. A top view is shown in bottom panel. Scale bar $10 \mu \mathrm{m}$. 
Embryos from the generated FMNL-GFP line were then collected, fixed and imaged. Figure 71 shows fixed embryos from FMNL-GFP flies during early, mid- and late cellularization in sagittal views and a top view (bottom panel), Dlg was used to stain lateral membranes and GFP booster to show GFP signal. I could detect a cytoplasmic, possibly vesicular staining $\mathrm{n}$ the GFP-channel, whereas I could not detect a clear membrane localization throughout cellularization.

\subsection{FMNL is expressed in larval and adult tissues}

To further analyze localization of FMNL-GFP, I dissected testes and ovaries of adult flies, fixed and stained with GFP-booster and DAPI, to show DNA. In Figure 72A wild type (upper panel) as a control and FMNL-GFP (lower panel) testes are shown. In FMNL-GFP testes I could detect a clear expression. Furthermore, I could detect a clear expression of FMNL-GFP (Figure 72B, upper panel) in female ovaries compared to wild type ovaries (Figure 72B, lower panel). Enriched FMNL-GFP at the nurse cells, that may be also localized at the membrane, was marked with a yellow arrowhead. 


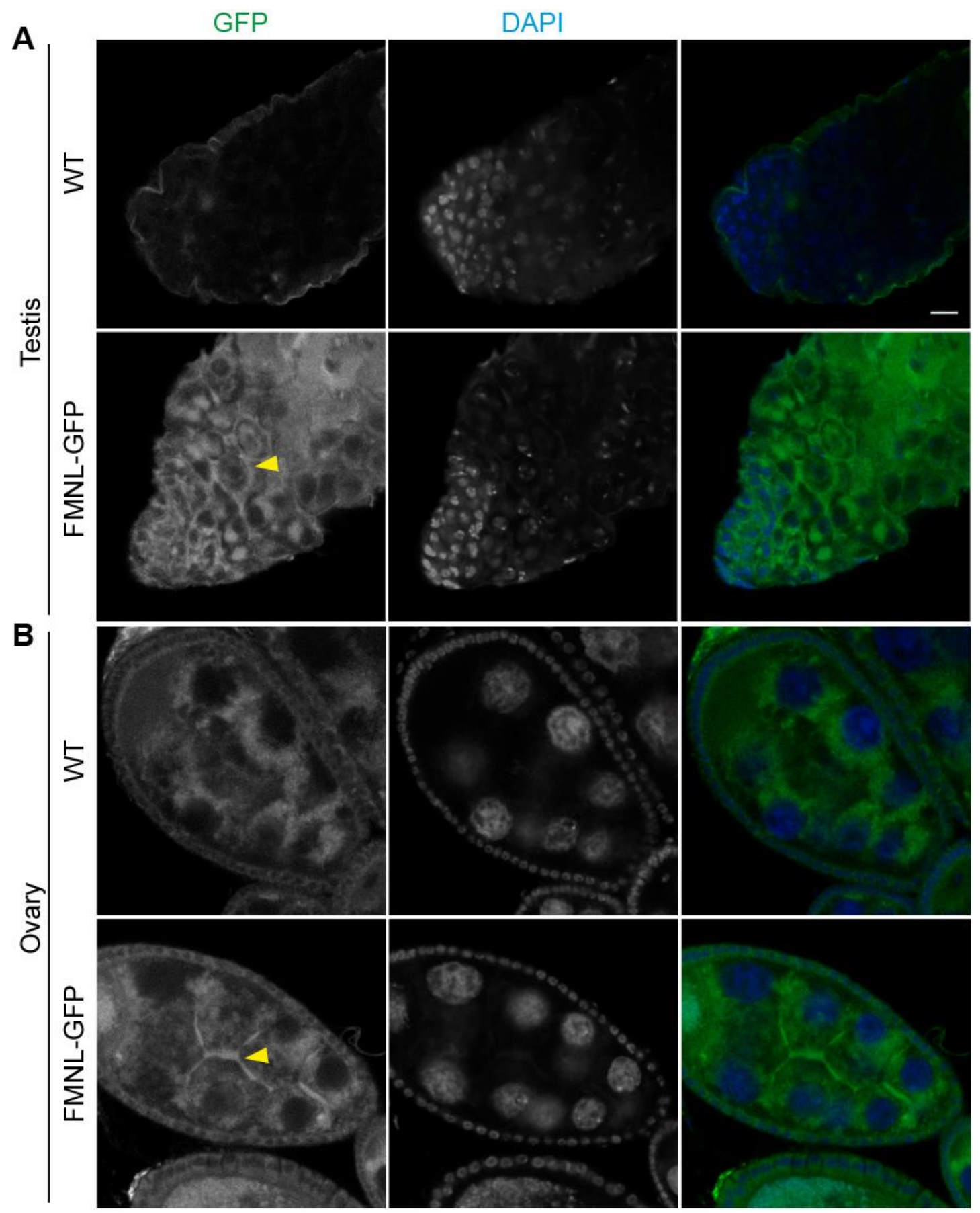

Figure 72 FMNL-GFP is expressed in testis and ovary.

(A-B) Images of testis (A) and ovary (B) of wild type and FMNL-GFP expressing flies stained with GFP-booster (grey/green) and DNA (grey/blue). Merged images are shown in right panel. (A) Wild type (upper panel) and FMNL-GFP (lower panel) testis. FMNL-GFP enrichment is indicated with yellow arrowhead. (B) Wild type (upper panel) and FMNL-GFP (lower panel) ovaries. WT ovary shows auto-fluorescence at nurse cells, FMNL-GFP enrichment between nurse cells is indicated with yellow arrowhead. Scale bar $10 \mu \mathrm{m}$.

I also screened several larval tissues for FMNL-GFP enrichment and I could detect high expression of FMNL-GFP in larval brains, which were also dissected, fixed and stained with GFP-booster and DAPI. Figure 73 shows brains from wild type compared to FMNL-GFP larval brains. Ventral nerve 
cord and optic lobes are shown in upper and lower panels as indicated. Some strong symmetric spots of FMNL-GFP could be detected at the ventral nerve cord and also some strong spots were visible in the optic lobe region. I was not able to detect these signals in wild type embryos.

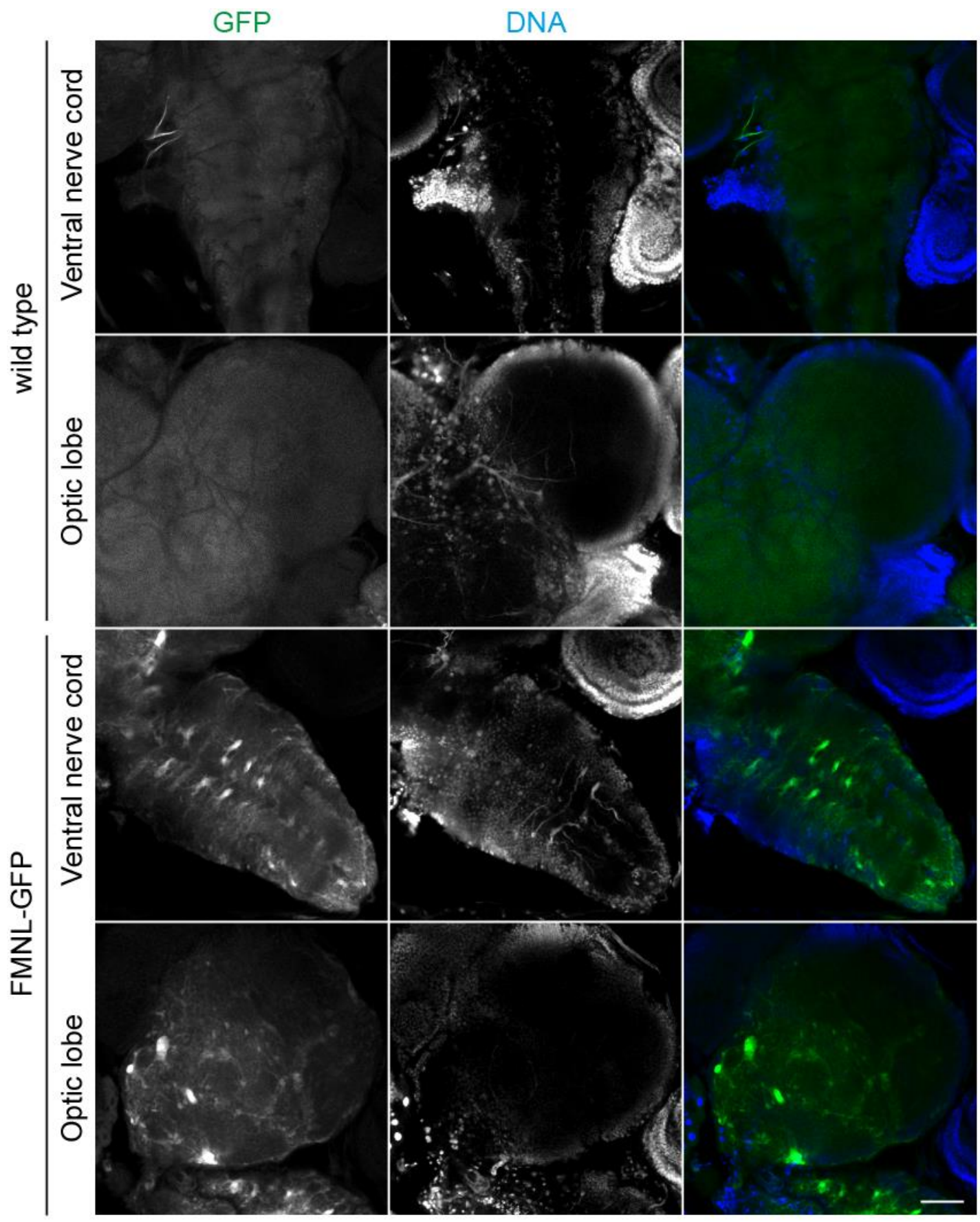

Figure 73 FMNL-GFP shows strong expression in larval brains.

(A-B) Images of fixed larval brains of (A) wild type and (B) FMNL-GFP expressing larval brains stained with GFP-booster (grey/green) and DNA (grey/blue). Merged images are shown in right panel. Ventral nerve cord (upper panel) and optic lobe (lower panel) are as indicated. Scale bar $20 \mu \mathrm{m}$. 
To further analyze larval brain FMNL-GFP expression on a cellular level, stainings were performed by Prof. Gaia Tavosanis/ Tomke Stürner (DZNE, Bonn) with an GFP antibody and Repo antibody, that marks glia cells. Indeed, FMNL-GFP was strongly enriched in glia cells at the ventral nerve cord and optic lobes, as shown in Figure 74.

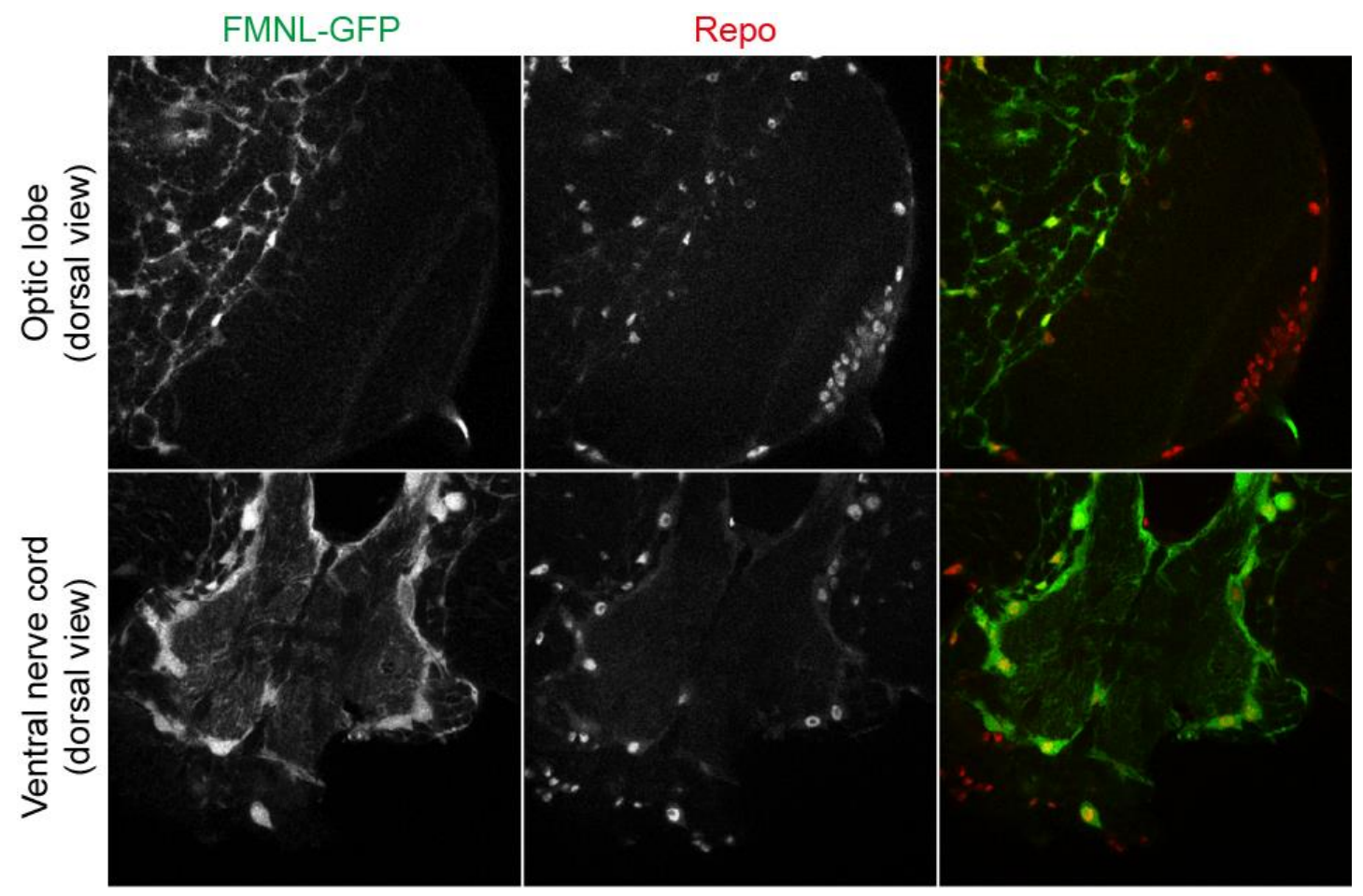

Figure 74 FMNL-GFP is enriched in glia cells in larval brains.

Images of fixed brains of FMNL-GFP (grey/green) expressing larvae stained against Repo (glia marker; grey/red). Upper panel shows optic lobe, lower panel ventral nerve cord. Images and stainings were done by Gaia Tavosanis/Tomke Stürner (DZNE/Bonn).

\section{FMNL-GFP can be pulled down from adult head extracts}

In order to perform further experiments to find possible binding partners of FMNL, I performed pull-down assays. However, I was not able to pull down FMNL-GFP from embryonic extracts in detectable amounts, possibly due to low expression levels. As the stainings of larval brains led to the hypothesis, that FMNL-GFP is expressed in higher levels in this tissue, I prepared extracts from adult heads (Figure 75). Before performing the pull-down assay, I made a Western Blot with extracts from adult heads, detecting FMNL-GFP with an antibody against GFP (Figure 75A). I could detect a band running at a size of $\sim 150 \mathrm{kDa}$ in extracts from FMNL-GFP expressing heads, that correlates with the predicted size of FMNL-GFP. However, a weaker band of this size could also be detected in extracts from wild type flies, possibly due to contamination. Furthermore, a second band was detected in heads from FMNL-GFP 
expressing flies, which could resemble a lower running isoform of FMNL-GFP or unspecific binding. In the pull-down assay, the extract of 200 adult heads from flies expressing FMNL-GFP was used and a western blot of samples from the input, the unbound fraction and the bound fraction was performed (Figure 75B). In this assay, FMNL-GFP was immunoprecipitated by GFP-binder beads. Like in Figure 75A, two bands could be detected in the input sample. In the unbound fraction, only the lower band could be detected which is a hint, that this was due to unspecific binding of the antibody. The bound fraction showed a clear band running at around $150 \mathrm{kDa}$ like the upper band in the input sample (Figure 75B).

From these results it would be possible to pull down FMNL-GFP from adult heads to further analyze binding partners of the protein f.e. by mass spectroscopy. However, these experiments were not part of my thesis.

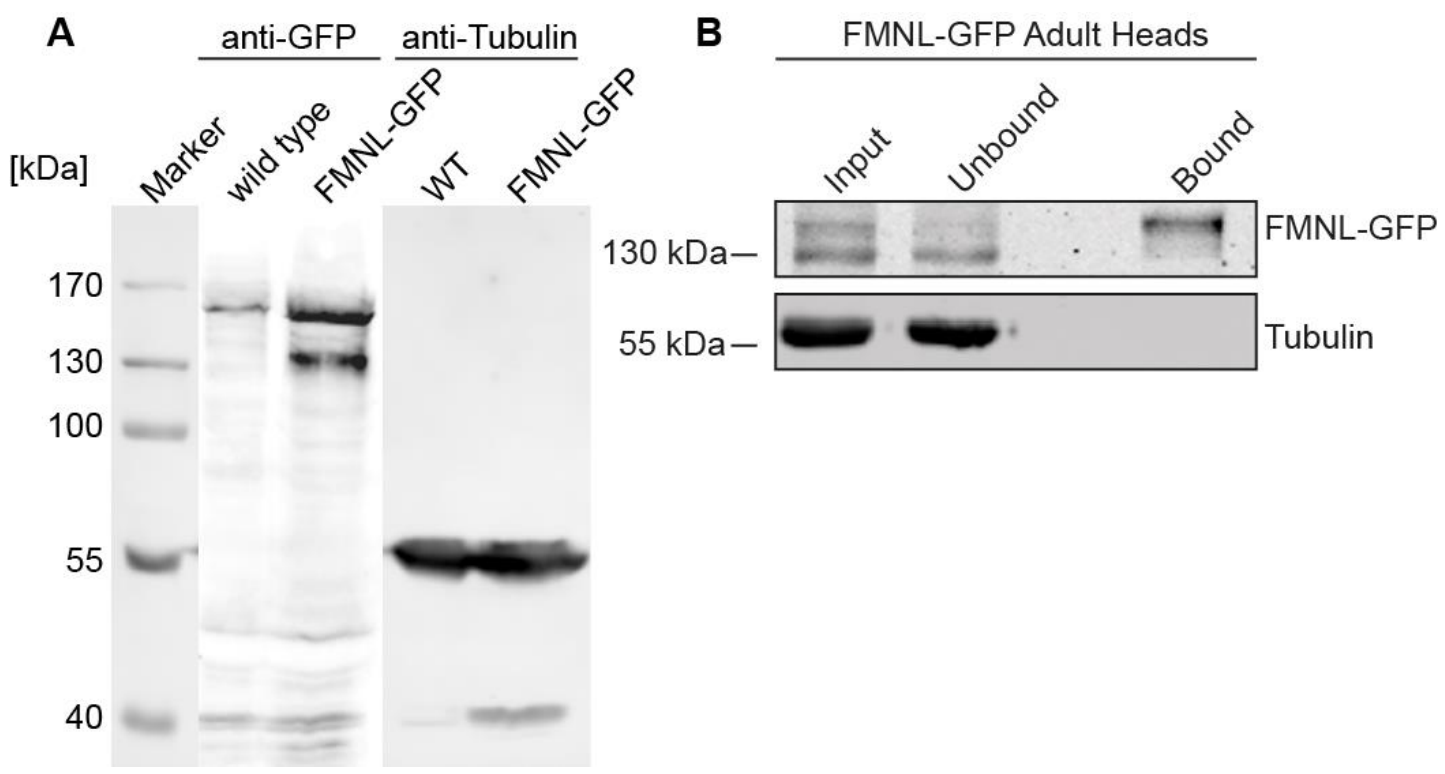

Figure 75 FMNL-GFP is expressed in adult heads.

(A-B) Western blot and immunoprecipitation of FMNL-GFP from adult heads. (A) Western blot of adult heads from wild type and FMNL-GFP expressing flies. 15 heads were loaded per lane. The membrane was stained with GFP antibody and Tubulin antibody as loading control. FMNL-GFP runs at $~ 150 \mathrm{kDa}$. (B) FMNL-GFP from 200 heads was immunoprecipitated with GFP-binder beads. Samples of input, unbound and bound fractions were loaded and stained with antibodies against GFP (upper panel) and Tubulin (lower panel) as loading control. 


\section{Discussion}

The aim of this study was to analyze the possible role of FMNL during early Drosophila embryogenesis by the generation of an FMNL mutant and FMNLGFP expressing transgenic flies. As FMNL was identified as a possible interaction partner of Slam in a yeast-two-hybrid screen and Slam itself plays a crucial role for the invagination of furrows during cellularization (Acharya et al., 2014; Lecuit et al., 2002), a role of FMNL for this process was possible. FActin itself is fundamental for the process of cellularization as no invagination of cellularization furrows takes place if actin is depolymerized by drugs (Sokac and Wieschaus, 2008b). Furthermore, another protein of the formin family, Dia, is needed for proper cellularization (Afshar et al., 2000) and formation of cortical domains, as I could show in part B of my thesis.

In this study the generation of a mutant for FMNL by imprecise excision of a p-element or by directed mutagenesis with different CRISPR methods did not lead to a visible phenotype. In 2016 Dollar et al. presented a study in which they generated three FMNL mutant fly lines by imprecise excision of a pelement, which were described to be homozygous lethal. However, two of these lines were homozygous viable in our lab. Both were balanced by the TM6B balancer whereas the lethal line was balanced by TM3. Possibly, balancing by TM6B was inefficient in this case as the mutations generated by Dollar et al (2016) were clearly characterized, the deletion of other genes rescued by adding back the deleted gene sequence other than FMNL and rescued by insertion of full-length FMNL sequence. However, the timepoint of lethality was not described in this study. The focus of Dollar et al. (2016) lay on the morphogenesis of ommatidial cells in eye development, rotation of ommatidial cells and the outgrowth of neurons during larval and pupal development. The effects on ommatidial rotation were weak but reproducible in the study of Dollar et al. (2016) and much stronger by the overexpression of dominant negative and constitutively active variants of FMNL. However, they analyzed the mutations and overexpression phenotypes by tissue specific expression. The FRT site that they introduced into their mutants to generate homozygous mutant clones did not work to generate maternal germ line clones and I did not follow up the analysis of this mutant.

The described phenotypes from Dollar et al. (2016) led to the hypothesis, that FMNL is needed for the morphogenesis of ommatidial cells but also plays a 
role in planar cell polarity (PCP) downstream of Cdc42. It was also described before, that PCP genes can act on axon growth and also there FMNL played a role. However, PCP genes are not in general needed for the formation of cortical domains and successful cellularization. A similar role for FMNL in PCP signaling is not described yet for the vertebrate system.

In vertebrates FMNL was described to influence actin dynamics in the formation of lamellipodia and with this also cell migration (Block et al., 2012; Kage et al., 2017b). Furthermore, the role of FMNL in epithelial formation was researched in 3D cell culture. Here it was shown, that it is necessary for the formation of adherens junctions and also for lumen formation (Grikscheit et al., 2015). As the formation of an epithelium is also happening during cellularization in Drosophila embryos a similar role for FMNL in this process would be consequential. Although FMNL was found in the Yeast-two-hybrid screen as potential binding partner of Slam, I was not able to show a function in cellularization, as the mutants that I generated did not show a phenotype. Another argument for a role of FMNL in cellularization is that I could show that FMNL was expressed during cellularization by in situ hybridization. In addition, I could detect FMNL-GFP during cellularization but I could not show an enrichment of the protein at the invaginating cellularization furrow but rather in the cytoplasm, possibly in vesicles. As vesicle transport and endocytosis are playing roles in successful cellularization (Fabrowski et al., 2013; Lecuit and Wieschaus, 2000), a function of FMNL in this process can still not be excluded. Another possibility to investigate a possible role for FMNL in cellularization would be the expression of different FMNL variants. As FMNL is autoinhibited the overexpression of a constitutively active version by deletion of the N-terminal regulatory domains could give rise to overexpression phenotypes. Also, the expression of a dominant negative version with deleted $\mathrm{FH} 2$ domain could rule out possible compensations of a FMNL mutation by other formins (Dollar et al., 2016).

Next to the expression of FMNL-GFP in the embryo, I also studied its localization in different larval and adult tissues. I could find FMNL-GFP to be expressed in the male testis and the female ovary, where I could also detect a possible accumulation at the membranes between the nurse cells. However, I did not follow up a possible function of FMNL in these tissues. I could also detect a high expression of FMNL-GFP in the larval brain. Together with stainings by Prof. Gaia Tavosanis and Tomke Stürner (DZNE/ Bonn) the cells 
expressing FMNL-GFP could be identified as glia cells. Glia cells belong to the most abundant cell type of the central nervous system (CNS) and play various roles not only in neuron function, health and regulation, they are also known to function in axon guidance (reviewed in Ou et al., 2014; Stork et al., 2012). Interestingly, Dollar et al. (2016) showed a function of FMNL in the CNS. In their study, they could show, that FMNL is, together with the formin DAAM, required for correct growth of axons in the mushroom body. Together with my result, that flies with reduced amounts of FMNL showed reduced fitness, a role for FMNL in the formation and function of the CNS is likely.

Taken together, although I was not able to describe a function for FMNL during cellularization, I was successful in the generation of a functional FMNL-GFP transgene, that gave the hint to a possible function for the CNS due to its expression in glia cells. Furthermore, this transgene could be used for further biochemical and functional analysis. The expression and purification of FMNL-GFP from flies could $\mathrm{f}$. e. be used for the analysis of binding partners by mass spectroscopy. Also, the subcellular localization of FMNL in glia cells could be investigated further and the role of FMNL for the formation and/ or maintenance of glia cells and its role in CNS development and function could be analyzed further. 


\section{Material and Methods}

\subsection{Materials}

\subsubsection{Reagents}

All standard reagents and chemicals were obtained from AppliChem $\mathrm{GmbH}$ (Darmstadt), Carl Roth GmbH (Karlsruhe), Gibco BRL (Eggenstein), Invitrogen (Carlsbad, USA), Merck (Darmstadt), Sigma-Aldrich (St. Louis, USA) or Thermo Fisher Scientific (Waltham, USA) unless otherwise mentioned.

\subsubsection{Enzymes}

Restriction enzymes were purchased from Thermo Fisher Scientific (Waltham, USA) or New England Biolabs (Ipswich, USA) and used as described by the manufacturer. The following other enzymes were used:

DNase I

Lysozyme

Proteinase $\mathrm{K}$

T7 RNA polymerase

TEV protease
(Roche, Basel, Switzerland)

(AppliChem GmbH, Darmstadt,

Germany)

(Roche, Basel, Switzerland)

(expressed and purified in the lab)

(expressed and purified in the lab of

Prof. Dirk Görlich)

Transcriptor Reverse Transcriptase (Roche, Basel, Switzerland)

\subsubsection{Commercial kits}

The following kits were used according to the instructions of the manufacturers:

HiPure Plasmid Mini Kit

Plasmid Midi Kit Nucleobond AX

iQ SYBR Green Supermix
(Life Technologies, Carlsbad, USA)

(Macherey-Nagel, Düren, Germany)

(Bio-Rad, München, Germany)

\subsubsection{Chromatography}

The following columns and materials were used for the purification of recombinant proteins:

CNBr-activated Sepharose 4B

HisTrap HP columns
(GE Healthcare Life Sciences, Little Chalfont, UK)

(GE Healthcare Life Sciences, Little Chalfont, UK) 
PD-10 desalting columns

(GE Healthcare Life Sciences, Little Chalfont, UK)

\subsubsection{Media}

LB and SOC media were prepared by lab technicians according to standard protocols as described in Sambrook and Russel, 2001.

\subsubsection{Microscopy}

\section{Confocal microscope LSM780}

equipped with an AiryScan Unit: Zeiss AxioObserver.Z1 (Carl Zeiss, Jena, Germany, 2011)

Detectors: $\quad$ PMT

GASP

Lasers: $\quad$ Diodelaser (405 nm, $30 \mathrm{~mW}$ )

AiryScan detection

Argonlaser $(458$ nm, 488 nm, 514 nm, 25

$\mathrm{mW})$

DPSS-Laser (561 nm, $20 \mathrm{~mW}$ )

HeNe-Laser (633 nm, $5 \mathrm{~mW}$ )

Objectives:

C-Apochromat (40x, NA 1.2, water)

Plan-Appochromat (63x, NA 1.4, oil)

LCI-Plan Neofluar (63x, NA 1.3, multiimmersion)

Two-Photon Microscope LSM (Carl Zeiss, Jena, Germany) $7 \mathrm{MP}$

Laser:

Mode-locked Ti-sapphire laser

(Coherent, Santa Clara, USA)

Objective:

Plan-Apochromat (20x, NA 1, water)

(Carl Zeiss, Jena, Germany)

\section{Other light microscopes:}

Microinjection microscope (Carl Zeiss, Jena, Germany)

Zeiss Axioplan 2 equipped with (Carl Zeiss, Jena, Germany) an AxioCam MRc

\section{Stereomicroscopes:}

Zeiss Stemi 2000

(Carl Zeiss, Jena, Germany) 


\subsubsection{Buffers and solutions}

All buffers were prepared according to Sambrook and Russels (2001) unless otherwise stated.

Table 1 Buffers and solutions

\begin{tabular}{|c|c|}
\hline Name & Composition \\
\hline \multicolumn{2}{|l|}{ General buffers and solutions } \\
\hline Phosphate buffered saline (PBS) & $\begin{array}{l}130 \mathrm{mM} \mathrm{NaCl} \\
7 \mathrm{mM} \mathrm{Na}_{2} \mathrm{HPO}_{4} \\
3 \mathrm{mM} \mathrm{NaH}_{2} \mathrm{PO}_{4} \\
\text { pH } 7.4\end{array}$ \\
\hline PBT & PBS with $0.2 \%$ Tween \\
\hline TE buffer & $\begin{array}{l}10 \mathrm{mM} \text { Tris/Hcl, } \mathrm{pH} 8 \\
1 \mathrm{mM} \text { EDTA }\end{array}$ \\
\hline Embryo fixation solution & $\begin{array}{l}4.5 \mathrm{~mL} \text { PBS } \\
0.5 \text { or } 1 \mathrm{~mL} \text { Formaldehyde (37\%) } \\
5 \mathrm{~mL} \text { Heptane }\end{array}$ \\
\hline Immunostaining blocking buffer & $\begin{array}{l}1 \times \text { PBS } \\
5 \% \text { BSA }\end{array}$ \\
\hline \multicolumn{2}{|c|}{ Buffers and solutions for SDS-PAGE, Western Blot and Immunoprecipitation } \\
\hline Stacking gel buffer & $\begin{array}{l}0.5 \mathrm{M} \text { Tris/HCl, } \mathrm{pH} 6.8 \\
0.4 \% \text { SDS }\end{array}$ \\
\hline Separating gel buffer & $\begin{array}{l}1.5 \mathrm{M} \text { Tris/HCl, } \mathrm{pH} 8.8 \\
0.4 \% \text { SDS }\end{array}$ \\
\hline $10 \times$ SDS-PAGE running buffer & $\begin{array}{l}0.25 \text { M Tris } \\
2 \mathrm{M} \text { glycine } \\
1 \% \text { SDS }\end{array}$ \\
\hline 6x Lämmli buffer & $\begin{array}{l}375 \mathrm{mM} \text { Tris/HCl } \\
10 \% \text { SDS } \\
50 \% \text { Glycerol } \\
0.6 \mathrm{M} \text { DTT } \\
0.06 \% \text { Bromphenole blue }\end{array}$ \\
\hline Wet transfer buffer & $\begin{array}{l}25 \text { mM Tris } \\
175 \text { mM Glycine } \\
20 \% \text { Methanol }\end{array}$ \\
\hline Blocking buffer & $\begin{array}{l}\text { 1x PBST } \\
5 \% \text { milk powder }\end{array}$ \\
\hline
\end{tabular}


Buffers and solutions - continuation

\begin{tabular}{|c|c|}
\hline RIPA Buffer & $\begin{array}{l}10 \mathrm{mM} \text { Tris/HCl, pH } 7.5 \\
150 \mathrm{mM} \mathrm{NaCl} \\
5 \mathrm{mM} \text { EDTA } \\
1 \% \text { Deoxycholate } \\
1 \% \text { Triton X-100 } \\
0.1 \% \text { SDS } \\
\text { Add fresh: } \\
1 \text { x Roche protease inhibitor cocktail } \\
2 \text { mM PMSF }\end{array}$ \\
\hline \multicolumn{2}{|c|}{ Buffers and solutions for purification of recombinant proteins } \\
\hline Lysis buffer & $\begin{array}{l}20 \mathrm{mM} \text { Na-Phosphate, } \mathrm{pH} 8 \\
50 \mathrm{mM} \mathrm{NaCl} \\
20 \mathrm{mM} \text { Imidazole }\end{array}$ \\
\hline Washing buffer & $\begin{array}{l}20 \mathrm{mM} \text { Na-Phosphate pH } 8.0 \\
500 \mathrm{mM} \mathrm{NaCl} \\
40 \mathrm{mM} \text { Imidazole }\end{array}$ \\
\hline Elution buffer & $\begin{array}{l}20 \mathrm{mM} \text { Na-Phosphate pH } 8.0 \\
500 \mathrm{mM} \mathrm{NaCl} \\
250 \mathrm{mM} \text { Imidazole }\end{array}$ \\
\hline TEV buffer & $\begin{array}{l}50 \mathrm{mM} \text { Tris, } \mathrm{pH} 8 \\
0.5 \mathrm{mM} \text { EDTA } \\
1 \mathrm{mM} \text { DTT }\end{array}$ \\
\hline \multicolumn{2}{|c|}{ Buffer and solutions for purification of antibodies } \\
\hline Coupling buffer & $\begin{array}{l}100 \mathrm{mM} \mathrm{NaHCO} / \mathrm{NaOH}, \mathrm{pH} 8.3 \\
500 \mathrm{mM} \mathrm{NaCl}\end{array}$ \\
\hline Blocking buffer & $0.1 \mathrm{mM}$ Tris/HCl, $\mathrm{pH} 8.0$ \\
\hline Washing buffer I & $\begin{array}{l}0.1 \mathrm{M} \mathrm{Na} \text {-actetate } \\
0.5 \mathrm{M} \mathrm{NaCl}, \mathrm{pH} 4.0\end{array}$ \\
\hline Washing buffer II & $\begin{array}{l}0.1 \mathrm{M} \text { Tris/HCl, pH } 8.0 \\
0.5 \mathrm{M} \mathrm{NaCl} ; \mathrm{PH} 8.0\end{array}$ \\
\hline High salt buffer & $\begin{array}{l}4 \mathrm{M} \mathrm{MgCl}_{2} \\
50 \mathrm{mM} \text { Tris/HCl, pH } 7.5\end{array}$ \\
\hline Low pH buffer & 50 mM Glycine, pH 2.5 \\
\hline High pH buffer & $50 \mathrm{mM}$ Triethanolamine, $\mathrm{pH} 11.5$ \\
\hline Neutralization buffer & 1 M Tris, $\mathrm{pH} 9$ \\
\hline
\end{tabular}


Buffers and solutions - continuation

\begin{tabular}{|c|c|}
\hline \multicolumn{2}{|c|}{ Buffers and solutions for in situ hybridization } \\
\hline NBT & $75 \mathrm{mg} / \mathrm{mL}$ in $70 \% \mathrm{DMF}$ \\
\hline BCIP & $50 \mathrm{mg} / \mathrm{mL}$ in DMF \\
\hline Hybridization solution & $\begin{array}{l}50 \% \text { Formamide } \\
5 \times \text { SSC } \\
50 \mu \mathrm{g} / \mathrm{mL} \text { Heparin } \\
0.2 \% \text { Tween } \\
100 \mu \mathrm{g} / \mathrm{mL} \text { tRNA } \\
\text { Adjust with water to final volume }\end{array}$ \\
\hline AP-Buffer & $\begin{array}{l}100 \mathrm{mM} \mathrm{NaCl} \\
50 \mathrm{mM} \mathrm{MgCl}_{2} \\
100 \mathrm{mM} \text { Tris, } \mathrm{pH} 9.5 \\
0.2 \% \text { Tween } \\
\text { Adjust with water to final volume }\end{array}$ \\
\hline NTP+Dig-labeling mix (10x) & $\begin{array}{l}10 \text { mM ATP } \\
10 \text { mM GTP } \\
10 \text { mM CTP } \\
6.5 \text { mM UTP } \\
3.5 \text { mM Dig-11-UTP, pH } 7.5\end{array}$ \\
\hline
\end{tabular}

For work with RNA, buffers were treated with Diethylpyrocarbonate (DEPC) as described in Sambrook and Russel, 2001

\subsubsection{Oligonucleotides}

All Oligonucleotides were purchased from Eurofins Genomics (Ebersberg, Germany).

Table 2 Oligunucleotides used in this study

\begin{tabular}{|l|l|l|}
\hline Name & Sequence $\mathbf{5}^{\prime} \rightarrow \mathbf{3}^{\prime}$ & Description \\
\hline AS12 & $\begin{array}{l}\text { GTA GCA ATG GAA CCG AGG } \\
\text { TC }\end{array}$ & Forward primer FMNL \\
\hline AS55 & AAT GGC TAG CTT GGA CGA GA & Reverse primer FMNL \\
\hline HS390 & CAC CGG TAT CGT TCT GGA CT & Forward primer Actin \\
\hline HS391 & $\begin{array}{l}\text { AGG GCA ACA TAG CAC AGC } \\
\text { TT }\end{array}$ & Reverse primer Actin \\
\hline
\end{tabular}




\subsubsection{Plasmids and fosmids}

Table 3 Plasmids and fosmids used in this study

\begin{tabular}{|c|c|c|}
\hline Name & Description & Source \\
\hline $\begin{array}{l}\text { pQE-ZZ-FMNL- } \\
\text { His }\end{array}$ & $\begin{array}{l}\text { C-terminal His-tag and N- } \\
\text { terminal ZZ-tag fused to N- } \\
\text { terminal half of FMNL }(1-596 \mathrm{aa})\end{array}$ & J. Großhans \\
\hline $\begin{array}{l}\text { FMNL cDNA } \\
\text { plasmid }\end{array}$ & $\begin{array}{l}\text { Plasmid pOTB7 containing the } \\
\text { cDNA of FMNL. \#AT04875 }\end{array}$ & DGRC \\
\hline FMNL-GFP fosmid & $\begin{array}{l}\text { Fosmid containing the genetic } \\
\text { region of FMNL tagged with a C- } \\
\text { terminal GFP. }\end{array}$ & $\begin{array}{l}\text { TransgeneOme } \\
\text { project/ Frank } \\
\text { Schnorrer }\end{array}$ \\
\hline
\end{tabular}

\subsubsection{Antibodies}

Table 4 Primary antibodies used in this study

\begin{tabular}{|c|c|c|c|c|}
\hline \multirow[t]{2}{*}{ Antibody } & \multirow[t]{2}{*}{ Raised in } & \multicolumn{2}{|c|}{ Dilution } & \multirow[t]{2}{*}{ Source } \\
\hline & & Staining & Western Blot & \\
\hline$\alpha$-Tubulin & Mouse & - & $1: 50000$ & $\begin{array}{l}\text { Sigma Aldrich (St. } \\
\text { Louis, USA) }\end{array}$ \\
\hline Armadillo & Mouse & $1: 50$ & - & $\begin{array}{l}\text { Hybridoma } \quad \text { Bank } \\
\text { N2 7A1 }\end{array}$ \\
\hline Canoe & Rabbit & 1:1000 & - & $\begin{array}{l}\text { Choi et al., 2013/ M. } \\
\text { Peifer }\end{array}$ \\
\hline $\mathrm{CpA}$ & Rabbit & $1: 200$ & - & $\begin{array}{l}\text { Amândio et al., } \\
\text { 2014/ F. Janody }\end{array}$ \\
\hline Bazooka & Rabbit & 1:1000 & - & A. Wodarz \\
\hline Dia & Guinea pig & 1:1000 & $1: 5000$ & J. Großhans \\
\hline Dia & Rabbit & 1:1000 & - & $\mathrm{ZMBH}$ \\
\hline Dlg & Mouse & $1: 100$ & - & $\begin{array}{l}\text { Hybridoma Bank } \\
\text { 4F3 }\end{array}$ \\
\hline FMNL & Guinea pig & 1:0000 & $\begin{array}{l}\text { Serum: 1:1000 } \\
\text { Purified: } \\
\text { 1:1000/1:500 }\end{array}$ & J. Großhans \\
\hline FMNL & Rabbit & $1: 0000$ & $\begin{array}{l}\text { Serum: 1:1000 } \\
\text { Purified: } \\
\text { 1:1000/1:500 }\end{array}$ & J. Großhans \\
\hline
\end{tabular}


Primary antibodies used in this study - continuation

\begin{tabular}{|l|l|l|l|l|}
\hline GFP & Rabbit & - & $1: 10000$ & $\begin{array}{l}\text { Torrey Pines Biolabs } \\
\text { (Seacaucus, USA) }\end{array}$ \\
\hline Slam & Guinea pig & $1: 10000$ & - & J. Großhans \\
\hline Slam & Rabbit & $1: 10000$ & - & J. Großhans \\
\hline Sponge & Guinea pig & $1: 1000$ & - & $\begin{array}{l}\text { Biersmith et al., } \\
2011 / \text { E. Geisbrecht }\end{array}$ \\
\hline
\end{tabular}

Secondary Alexa-conjugated antibodies were used at a final concentration of $4 \mu \mathrm{g} / \mathrm{mL}$ (1:500). Alexa-conjugated Phalloidin for staining of F-actin was used at a dilution of 1:1000. Both products were purchased from Invitrogen (Carlsbad, USA). GFP-booster-Atto488 was used at a final concentration of $2 \mu \mathrm{g} / \mathrm{mL}$ (1:500) and purchased from Chromotek (Martinsried, Germany). Anti-Digoxigenin-peroxidase antibodies were purchased from Roche (Basel, Switzerland) and used at a final dilution of 1:200. As secondary antibodies for western blots IRDye-800CW and IRDye-680 were used at a final concentration of $0.05 \mu \mathrm{g} / \mathrm{mL}$ (1:20000) and purchased from LI-COR Biotechnology (Bad Homburg, Germany).

\subsubsection{Fly stocks}

If not otherwise stated, fly stocks were obtained from Bloomington Drosophila stock center.

Table 5 Fly stocks used in this study

\begin{tabular}{|c|c|c|}
\hline Name & Genotype & Source \\
\hline $\begin{array}{l}\text { Wild type / } \\
\text { OrR }\end{array}$ & $\frac{+}{+}$ & \\
\hline 117GFP & 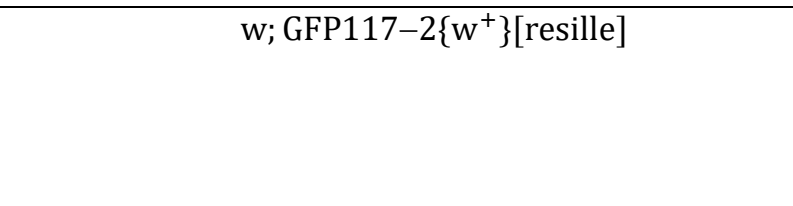 & $\begin{array}{l}\text { J. Zallen/ } \\
\text { Blankenship } \\
\text { et al., } 2006\end{array}$ \\
\hline $\begin{array}{l}\text { UAS- } \\
\text { BazGFP }\end{array}$ & $\mathrm{w} ; \frac{\text { tub-Gal4-VP16 }\left\{\mathrm{w}^{+}\right\}[15], \mathrm{UASp}-\mathrm{bazGFP}\left\{\mathrm{w}^{+}\right\}}{\mathrm{TM} 6 \mathrm{~B}}$ & $\begin{array}{l}\text { D. St } \\
\text { Johnston/ } \\
\text { Benton and } \\
\text { Johnston, } \\
2003\end{array}$ \\
\hline BazGFP & y w P $\{\mathrm{w}[+\mathrm{mC}]=\mathrm{PTT}-\mathrm{GC}\} \mathrm{baz}[\mathrm{CC} 01941]$ & $\begin{array}{l}\text { Bloomingto } \\
\mathrm{n} \# 51572\end{array}$ \\
\hline
\end{tabular}


Fly stocks used in this study - continuation

\begin{tabular}{|c|c|c|}
\hline baz RNAi & 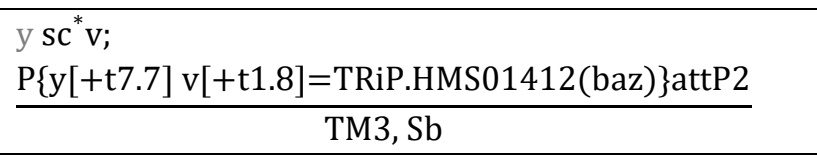 & $\begin{array}{l}\text { Bloomingto } \\
\text { n \#35002 }\end{array}$ \\
\hline canoe & $\frac{\text { FRT82B cno }^{\text {R2 }}}{\text { TM3 twiGFP }}$ & $\begin{array}{l}\text { Choi et al., } \\
\text { 2013/ M. } \\
\text { Peifer }\end{array}$ \\
\hline canoeYFP & PBac $\left\{602\right.$. P. SVS-1\}cno ${ }^{\text {CPTI000590 }}$ & $\begin{array}{l}\text { Kyoto Stock } \\
\text { Center } \\
\# 115111\end{array}$ \\
\hline Cherry-slam & w; USAp-Chery-slam $\left\{\mathrm{w}^{+}\right\}$ & $\begin{array}{l}\text { Acharya et } \\
\text { al., } 2014\end{array}$ \\
\hline dia & $\mathrm{w} ; \frac{\operatorname{dia}\left[\text { sy5]FRT[2L] } \text { neo }^{\mathrm{R}}\right\}}{\text { Cyo, hs-hid }\left\{\mathrm{w}^{+}\right\}}$ & $\begin{array}{l}\text { Afshar et al., } \\
2000\end{array}$ \\
\hline dia 117GFP & $\mathrm{w} ; \frac{\mathrm{GFP} 117-2\left\{\mathrm{w}^{+}\right\}[\text {resille }] \text { dia }[\text { sy5 }] \mathrm{FRT}[2 \mathrm{~L}]\left\{\mathrm{neo}^{\mathrm{R}}\right\}}{\mathrm{CyO}}$ & J. Großhans \\
\hline$d z y^{\Delta 8}$ & $\mathrm{w} ; \frac{\mathrm{dzy}[\Delta 8] \mathrm{b} \text { pr FRT2L }}{\mathrm{SM} 1}$ & $\begin{array}{l}\text { Huelsmann } \\
\text { et al., } 2006\end{array}$ \\
\hline ELMO & $\mathrm{w} ; \frac{\text { al dp elmo[367]b pr FRT }[40 \mathrm{~A}]\left\{\mathrm{neo}^{\mathrm{R}}\right\}}{\text { CyO, hs-hid }\left\{\mathrm{w}^{+}\right\}}$ & $\begin{array}{ll}\text { Winkler et } \\
\text { al., 2015/ F. } \\
\text { Schnorrer }\end{array}$ \\
\hline ELMO-GFP & $\mathrm{w} ;$; ELMO-GFP $\left\{\mathrm{w}^{+}\right\}$ & $\begin{array}{lr}\text { Z. } & \text { Lv; } \\
\text { Schmidt } & \text { et } \\
\text { al., } 2017 & \end{array}$ \\
\hline FMNL-GFP & $\mathrm{w} ;$; attP-B3 [FMNL - GFP $\left.{ }^{\mathrm{Fos}}\right]$ & $\begin{array}{l}\text { Generated in } \\
\text { this work }\end{array}$ \\
\hline $\begin{array}{l}\text { FMNL } \quad \mathrm{p}^{-} \\
\text {element }\end{array}$ & $\mathrm{y} w ; \frac{\mathrm{P}\{\mathrm{GSV} 7\} \mathrm{GS} 23052}{\mathrm{TM} 3, \mathrm{Sb} \text { Ser }}$ & $\begin{array}{l}\text { Kyoto Sock } \\
\text { Center } \\
\# 204678\end{array}$ \\
\hline FMNL (Def) & $\mathrm{w}^{1118} ; \frac{\mathrm{Df}(3 \mathrm{~L}) \mathrm{ED} 4528}{\mathrm{TM} 6 \mathrm{C}, \mathrm{Sb} \mathrm{c}}$ & $\begin{array}{l}\text { Kyoto Stock } \\
\text { Center } \\
150136\end{array}$ \\
\hline $\begin{array}{l}\text { myoII3xGF } \\
P\end{array}$ & MyoII :: 3xGFP & $\begin{array}{l}\text { Y. Bellaîche/ } \\
\text { Pinheiro et } \\
\text { al., } 2017\end{array}$ \\
\hline mtd-Gal4 & $\begin{array}{c}\mathrm{P}\left[\text { otu-Gal4::VP16.R]1, } \mathrm{w}^{*} ; \mathrm{P}[\text { Gal4-nos.NGT }] 40 ;\right. \\
\text { P[Gal4::VP16-nos.UTR]CG6325(MVD1) }\end{array}$ & $\begin{array}{l}\text { Bloomingto } \\
\mathrm{n} \# 31777\end{array}$ \\
\hline
\end{tabular}

Fly stocks used in this study - continuation 


\begin{tabular}{|c|c|c|}
\hline nanos $\varphi$ C31 & $\mathrm{y}^{1} \mathrm{w}^{1118} ; \mathrm{PBac}\left\{\mathrm{y}^{+}-\mathrm{attP}-\mathrm{B}\right\} 3 \mathrm{VK} 00033$ & F. Schnorrer \\
\hline $\begin{array}{l}\text { nlsGFP } \\
\text { FRT[2L] }\end{array}$ & 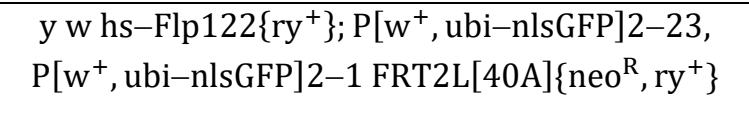 & S. Luschnig \\
\hline Rap1 & y w hsFLP; $\frac{\operatorname{Rap}^{\mathrm{P}(5709)} \text { FRT[2A] }}{\mathrm{TM} 3, \mathrm{Sb}}$ & $\begin{array}{l}\text { R. Reuter/ } \\
\text { Knox and } \\
\text { Brown, } 2002\end{array}$ \\
\hline GFP-Rap1 & $\mathrm{w} ; \frac{\mathrm{GFP}-\mathrm{Rap} 1}{\mathrm{CyO}}$ & $\begin{array}{lr}\text { E. } & \text { Knust/ } \\
\text { Knox } & \text { and } \\
\text { Brown, } & 2002\end{array}$ \\
\hline scribble & $\mathrm{w} ; \frac{\mathrm{scrib}^{1} \mathrm{FRT} 82 \mathrm{~B}}{\mathrm{TM} 3, \mathrm{Sb}}$ & $\begin{array}{l}\text { D. Bilder/ } \\
\text { Bilder and } \\
\text { Perrimon, } \\
2000\end{array}$ \\
\hline scribbleGFP & w; scrib-GFP ${ }^{\mathrm{CA07683}}$ & $\begin{array}{l}\text { Buszczak et } \\
\text { al., } 2007\end{array}$ \\
\hline shibire $^{\mathrm{TS}}$ & $\frac{\mathrm{w} \text { shi }[1]}{\mathrm{FM} 6, \mathrm{y} B}$ & $\begin{array}{l}\text { Bloomingto } \\
\mathrm{n}\end{array}$ \\
\hline slam & $\mathrm{w} ; \frac{\operatorname{Df}(2 \mathrm{~L}) \operatorname{slam}\left\{\mathrm{w}^{+}\right\} \text {FRT }[2 \mathrm{~L}]\left\{\mathrm{neo}^{\mathrm{R}}\right\} \text { c slam } 5^{\prime}\left\{\mathrm{w}^{+}\right\}}{\mathrm{CyO}}$ & P. Wenzl \\
\hline sponge & $\frac{\text { ru st e spg[242]ca }}{\text { TM6, Ubx Abd-lacZ }}$ & $\begin{array}{l}\text { E. Schejter; } \\
\text { Postner et } \\
\text { al., } 1992\end{array}$ \\
\hline sponge (Df) & $\frac{\mathrm{Df}(3 \mathrm{R}) 3450}{\mathrm{TM} 6 \mathrm{~B}, \mathrm{Hu}}$ & E. Schejter \\
\hline TM3/TM6B & $\mathrm{w} ; \frac{\mathrm{TM} 3, \mathrm{Sb} \mathrm{Ser}}{\mathrm{TM} 6 \mathrm{~B}, \mathrm{~Tb} \mathrm{Hu}}$ & $\begin{array}{l}\text { Bloomingto } \\
\mathrm{n}\end{array}$ \\
\hline ovoD2L & hs-Flp[122]; $\frac{\text { ovoD FRT2L[40A] }}{\text { If / CyO, hs-hid }}$ & J. Großhans \\
\hline ovoD2R & hs-Flp[122]; $\frac{\text { FRT2R[42A] ovoD }}{\text { If / CyO, hs-hid }}$ & J. Großhans \\
\hline Rho-sensor & $\begin{array}{l}\text { attp40 [nanos } \\
:: \text { tdTomato-RBD-P2A-tdkatushk2a-CAAX] }\end{array}$ & R. Lehmann \\
\hline
\end{tabular}

\subsubsection{Bacterial strains}

Amplification of DNA plasmids was performed in E. coli DH5 $\alpha$. For expression of recombinant proteins E. coli BL21DE3 was used. 


\subsubsection{Equipment}

Äkta pure

EmulsiFlex-C5 Microfluidiser

FemtoJet Microinjector

Odyssey CLx Infra-red imaging system

P-87 Flaming/Brown Micropipette

Puller

qPCR cycler CFX96

Semi-Dry Transfer Cell

Thermo cycler

\subsubsection{Other materials}

10S and 3S VoltaLef Halocarbon oil

$\alpha$-Amanitin

Aquapolymount

Complete Mini (EDTA-free) Protease

Inhibitor Cocktail

Coverslips

Eppendorf tubes

Falcon tubes

Fly vials

GFP-Trap

Glass slides

Glass pipettes

Micropipettes

Micropipette tips

Minisart Single Use Filter Units $0.22 \mu \mathrm{m}$

Nitrocellulose membrane Protran

$0.45 \mu \mathrm{m} \mathrm{NC}$

PCR tubes

Petri dishes
(GE Healthcare Life Sciences, Little

Chalfont, UK)

(Avestin, Ottawa, Canada)

(Eppendorf, Hamburg, Germany)

(LI-COR Biosciences, Bad Homburg, Germany)

(Sutter Instrument Co., Novato, USA)

(Bio-Rad, München, Germany)

(Bio-Rad, München, Germany)

(Bio-Rad, München-Germany)

(Lehmann \& Voss \& Co., Hamburg, Germany)

(AppliChem, Darmstadt, Germany)

(Polysciences Inc., Warrington, USA)

(Roche, Basel, Switzerland)

(Thermo Fischer, Braunschweig, Germany)

(Eppendorf, Hamburg, Germany)

(BD, Heidelberg, Germany)

(Greiner, Kremsmünster, Austria)

(Chromotek, Martinsried, Germany)

(Thermo Fischer, Braunschweig,

Germany)

(Brand, Wertheim, Germany)

(Gilson, Middleton, USA)

(Sarstedt, Nürnbrecht, Germany)

(Sartorius, Göttingen, Germany)

(GE Healthcare Life Sciences, Little Chalfont, UK)

(Brand, Wertheim, Germany)

(Greiner, Kremsmünster, Austria) 
Pasteur pipettes

Pipet-aid

Protein concentrator

Ribolock RNase inhibitor

Safe-Seal RNase-free tips

Whatman $3 \mathrm{~mm}$ blotting paper

\subsubsection{Software}

Excel

Word

Powerpoint

Illustrator CS6

Photoshop CS6

ImageJ

Lasergene

Zen 2012
(Brand, Wertheim, Germany)

(Drummond, Birmingham, USA)

(Sartorius, Göttingen, Germany)

(Thermo Fischer, Braunschweig,

\section{Germany)}

(Biozym, Wien, Austria)

(GE Healthcare Life Sciences, Little Chalfont, UK)

(Microsoft, Redmond, USA)

(Microsoft, Redmond, USA)

(Microsoft, Redmond, USA)

(Adobe, San Jose, USA)

(Adobe, San Jose, USA)

(NIH, Bethesda, USA)

(GATC Biotech, Konstanz,

Germany)

(Carl Zeiss, Jena, Germany) 


\subsection{Methods}

Standard procedures that are not mentioned in the following sections were performed according to Sambrook and Russel, 2001

\subsubsection{DNA and RNA methods}

Amplification and purification of fosmid DNA

The fosmid with the whole genomic sequence of FMNL tagged with a GFP was provided by Frank Schnorrer/TransgeneOme. To amplify the fosmid, a preculture of $2 \mathrm{~mL}$ LB medium + chloramphenicol $(25 \mu \mathrm{g} / \mathrm{mL})$ was inoculated with the fosmid clone from a glycerol stock and grown over night at $25^{\circ} \mathrm{C}$. The preculture was then diluted to $10 \mathrm{~mL}$ (9 mL LB medium + chloramphenicol and $1 \mathrm{~mL}$ preculture) and $10 \mu \mathrm{L}$ of $10 \%$ Arabinose (final concentration $0.01 \%$ ) was added to induce high copy number. The culture was incubated at $37^{\circ} \mathrm{C}$ for 5 hours. The bacteria were harvested by centrifugation for $15 \mathrm{~min}$ with $6,000 \mathrm{rpm}$ at $4{ }^{\circ} \mathrm{C}$, the supernatant was discarded and the pellet stored at $20^{\circ} \mathrm{C}$.

For mini preparation of fosmid DNA, the HiPure Plasmid Miniprep Kit was used. Pipetting of the fosmid was avoided. For the procedure buffers E4 was prewarmed to $50{ }^{\circ} \mathrm{C}$ and buffer $\mathrm{L} 7$ to $37^{\circ} \mathrm{C}$ and the column was equilibrated with $2 \mathrm{~mL}$ EQ1. The cells were resuspended with $0.4 \mathrm{~mL} \mathrm{R3}+\mathrm{RNaseA}$ and lysed by addition of $0.4 \mathrm{~mL} \mathrm{L7}$, inverting of the tube for 5 times and incubation for $4 \mathrm{~min}$ at room temperature. For neutralization $0.4 \mathrm{~mL}$ of N3 was added, the tube inverted and incubated tor $4 \mathrm{~min}$ on ice before centrifugation at maximum speed for $10 \mathrm{~min}$ at $4{ }^{\circ} \mathrm{C}$. The supernatant was then loaded onto a column and washed twice with $2.5 \mathrm{~mL}$ W8. The DNA was eluted with $850 \mu \mathrm{L}$ E4 and precipitated with $595 \mu \mathrm{L}$ isopropanol and then centrifuged for $10 \mathrm{~min}$ at $4{ }^{\circ} \mathrm{C}$ with maximum speed. The supernatant was removed and the pellet washed with $800 \mu \mathrm{L} 70 \%$ ethanol. The pellet was air dried for $4 \mathrm{~min}$ and resuspended in $20 \mu \mathrm{L}$ TE buffer. For injection the concentration was freshly adjusted to $250 \mathrm{ng} / \mu \mathrm{L}$ with water.

Amplification and purification of plasmid DNA

Amplification and purification of plasmid DNA was done as described in Sambrook and Russel, 2001.

Isolation and purification of total RNA from embryos 
Isolation of total RNA from WT and P/Def embryos for quantitative real-time PCR. The embryos were collected over night on apple juice agar plates, dechorionated, transferred to Eppendorf tubes, weighed, frozen in liquid nitrogen and stored at $-80^{\circ} \mathrm{C}$.

For preparation of embryo lysates $\sim 12 \mathrm{mg}$ of P/Def and $\sim 11.8 \mathrm{mg}$ of WT embryos were used. The embryos were lysed with a micro pestle and $800 \mu \mathrm{L}$ of Trizol were added little by little to wash the pestle. The extract was incubated for $5 \mathrm{~min}$ at room temperature. After adding $160 \mu \mathrm{L}$ Chloroform, the extracts were shaken and after 2-3 min incubation at room temperature, centrifuged with $14,000 \mathrm{rpm}$ for $15 \mathrm{~min}$ at $4{ }^{\circ} \mathrm{C}$. The upper aqueous phase was transferred to a new Eppendorf tube and $400 \mu \mathrm{L}$ Isopropanol was added to precipitate RNA. The solution was incubated at room temperature for $10 \mathrm{~min}$ and then centrifuged for $30 \mathrm{~min}$ with $14,000 \mathrm{rpm}$ at $4^{\circ} \mathrm{C}$. The pellet was then washed with $70 \%$ ethanol (DEPC) and centrifuged for 10 min with 14,000 rpm at $4{ }^{\circ} \mathrm{C}$. The pellet was tried at room temperature under the hood and then resuspended in $50 \mu \mathrm{L}$ water (DEPC).

Production of cDNA by reverse transcription

$1 \mu \mathrm{g}$ of RNA sample was mixed with $1 \mu \mathrm{L}$ Oligo(dT) and adjusted with water to $13 \mu \mathrm{L}$. The sample was incubated in the PCR machine for $10 \mathrm{~min}$ at $65^{\circ} \mathrm{C}$ and then immediately cooled down on ice. The following master mix was prepared and added to the sample:

5x Reverse transcriptase buffer:

RNase inhibitor:

dNTP mix (10 mM):

Reverse transcriptase:
$4 \mu \mathrm{L}$

$0.5 \mu \mathrm{L}$

$2 \mu \mathrm{L}$

$0.5 \mu \mathrm{L}$

The samples were then incubated in the PCR machine with the following program: $30 \mathrm{~min}$ at $55^{\circ} \mathrm{C} ; 10 \mathrm{~min}$ at $85^{\circ} \mathrm{C}$. The cDNA was the stored at $-20^{\circ} \mathrm{C}$.

Quantitative Real-Time-PCR

A master mix with primers for FMNL and one with primers for actin as control were made as described by the manufacturer. The mix for one sample was as followed:

SYBR qPCR Mix $\mathrm{ddH}_{2} \mathrm{O}$
$12.5 \mu \mathrm{L}$

$8 \mu \mathrm{L}$ 


$\begin{array}{ll}\text { Primer } 1 & 1.25 \mu \mathrm{L} \\ \text { Primer } 2 & 1.25 \mu \mathrm{L} \\ \text { cDNA } & 2 \mu \mathrm{L}\end{array}$

$2 \mu \mathrm{L}$ of the mix were then transferred into a qPCR plate and incubated in the qPCR machine with the following program:

1. Initial $95^{\circ} \mathrm{C}, 3 \mathrm{~min}$ denaturation

2. Denaturation $95{ }^{\circ} \mathrm{C}, 10 \mathrm{~s}$

3. Annealing $54{ }^{\circ} \mathrm{C}, 15 \mathrm{~s}$

4. Elongation $72{ }^{\circ} \mathrm{C}, 30 \mathrm{~s}$

5. Melting curve $55^{\circ} \mathrm{C}-95^{\circ} \mathrm{C}, 10 \mathrm{~s}$ (increase $0.5{ }^{\circ} \mathrm{C}$ )

Steps $2-4$ were cycled 40 times.

The following samples were loaded as 2 duplicates:

- WT cDNA with primers for FMNL - WT cDNA with primers for actin - $p /$ Def cDNA with primers for $\quad$ - p/Def cDNA with primers for actin FMNL

- $\mathrm{H}_{2} \mathrm{O}$ with primers for FMNL $\quad-\mathrm{H}_{2} \mathrm{O}$ with primers for actin

- WT RNA with primers for FMNL - WT RNA with primers for actin

- P/Def RNA with primers for $\quad$ - P/Def RNA with primers for actin FMNL

For RNA samples the produced RNA was diluted like for the reverse transcription. For FMNL sample primers AS12 and AS55 and for actin sample primers HS390 and HS391 were used.

\subsubsection{Protein methods}

Isolation of total protein from embryos or adult heads

For embryonic extracts, staged embryos were collected from apple juice agar plates, dechorionized, transferred to Eppendorf tubes, weighted, immediately frozen in liquid nitrogen and stored at $-80^{\circ} \mathrm{C}$. To prepare extracts, the embryos were thawed, $1 \times$ Lämmli buffer was added ( $20 \mu \mathrm{L}$ per $1 \mathrm{mg}$ embryos), treated with a micro pestle and incubated at $95{ }^{\circ} \mathrm{C}$ for $10 \mathrm{~min}$. A suitable number of embryos (1 mg corresponds to 100 embryos) was loaded on an SDS-gel.

For extracts from adult heads, 50 adult flies were collected in $15 \mathrm{~mL}$ Falcon tubes and frozen in liquid nitrogen, shaken vigorously to dissociate heads 
from thoraxes and then put on a sieve to collect heads, which were transferred into an Eppendorf tube and stored at $-80^{\circ} \mathrm{C}$ or pestled in $30 \mu \mathrm{L} 1 \mathrm{x}$ Lämmli buffer. For western blot the embryo extract was incubated at $95{ }^{\circ} \mathrm{C}$ for $10 \mathrm{~min}$.

\section{Immunoprecipitation}

To prepare an extract from the adult heads, 200 heads were dounced in a precooled glass homogenizer in $500 \mu \mathrm{L}$ RIPA buffer, centrifuged for $15 \mathrm{~min}$ with $14,500 \mathrm{rpm}$ at $4{ }^{\circ} \mathrm{C}$. The supernatant was transferred to a new Eppendorf tube and an input sample was taken. To prepare the Sepharose-GFP-Trap beads, the tube was vortexed and $20 \mu \mathrm{L}$ were transferred into $500 \mu \mathrm{L}$ cold RIPA buffer and centrifuged for $2 \mathrm{~min}$ with $800 \mathrm{rpm}$ at $4{ }^{\circ} \mathrm{C}$. The supernatant was discarded and the procedure repeated twice. The lysate was then loaded onto the beads and incubated for $1 \mathrm{~h}$ at $4{ }^{\circ} \mathrm{C}$. Afterwards an unbound sample was taken from the supernatant. The beads were then washed three times with $1 \mathrm{~mL}$ RIPA buffer (centrifugation for $2 \mathrm{~min}$ with $800 \mathrm{rpm}$ at $4^{\circ} \mathrm{C}$ ). The beads were then eluted in $20 \mathrm{~mL}$ Lämmli buffer and incubated at $97^{\circ} \mathrm{C}$ for $10 \mathrm{~min}$ and stored at $-20^{\circ} \mathrm{C}$. The samples were then used to perform western blotting. SDS-PAGE and western blot

SDS-PAGE and western blotting were done according to standard procedures described in Sambrook and Russel, 2001.

After the blotting procedure, the membrane was blocked in PBT $+5 \%$ milk powder. The membrane was incubated with the primary antibody overnight at $4{ }^{\circ} \mathrm{C}$ or for $2 \mathrm{~h}$ at room temperature and then washed 4 times for $5 \mathrm{~min}$ before incubation with secondary antibody for $2 \mathrm{~h}$ at room temperature and afterwards washed as described before.

\subsubsection{Expression and purification of recombinant proteins}

\section{Large Scale protein expression}

For preincubation one colony from the LB plate was picked with a pipet tip and added to $20 \mathrm{~mL}$ LB medium with ampicillin $(100 \mu \mathrm{g} / \mathrm{mL})$ which was incubated over night at $37^{\circ} \mathrm{C}$. For large scale expression $10 \mathrm{~mL}$ of the preculture was added to $1 \mathrm{~L} \mathrm{LB}$ medium with Ampicillin and incubated at $37^{\circ} \mathrm{C}$ until an OD of 0.5 was reached. Before incubation a sample of $1 \mathrm{~mL}$ was taken, centrifuged for $1 \mathrm{~min}$ with 12,000 rpm at room temperature and the pellet was suspended in $1 x$ Lämmli buffer (100 $\mu \mathrm{L}$ per 1 OD) and stored at $20{ }^{\circ} \mathrm{C}$ as uninduced sample. The culture was then cooled down, expression 
was induced with $0.2 \mathrm{mM}$ IPTG and shaken at $18^{\circ} \mathrm{C}$ over night. Another sample was taken afterwards as induced sample.

Lysis of bacteria

The overnight culture was centrifuged at $4,000 \mathrm{k}$ for $20 \mathrm{~min}$ at $4{ }^{\circ} \mathrm{C}$ and the pellet was solubilized in $20 \mathrm{~mL}$ lysis buffer and stored at $-20^{\circ} \mathrm{C}$. The cells were thawed and $200 \mu \mathrm{L}$ Lysozyme, $200 \mu \mathrm{L}$ PMSF (1 mM) and DNase was added and incubated for $35 \mathrm{~min}$ on ice. The cells were lysed in a microfluidizer and then centrifuged in Beckmann centrifuge tubes for $15 \mathrm{~min}$ with15,000 k at $4{ }^{\circ} \mathrm{C}$ twice. A sample was taken from the pellet (insoluble fraction) and from supernatant (soluble fraction), mixed with Lämmli Buffer and stored at $-20{ }^{\circ} \mathrm{C}$.

Purification of recombinant proteins

All buffers were sterile filtrated and precooled before purification at $4{ }^{\circ} \mathrm{C}$. The Äkta was washed with sterile water and the columns were equilibrated with lysis buffer. The sample was loaded into a super loop and connected to the machine. The sample was loaded onto the His-Trap column with a flow rate of $0.7 \mathrm{~mL} / \mathrm{min}$. The flow-through was collected in $10 \mathrm{~mL}$ fractions. After loading the column was washed with washing buffer with a flow rate of $1 \mathrm{~mL} / \mathrm{min}$ until OD A280 was stable. Elution was done with elution buffer with a flow rate of $1 \mathrm{~mL} / \mathrm{min}$ and collected in fractions of $3 \mathrm{~mL}$. Afterwards the Äkta and column were washed with water and $20 \%$ ethanol.

TEV cleavage of ZZ-Tag

For cleavage of the ZZ-Tag $7 \mathrm{~mL}$ of purified protein solution was mixed with $100 \mu \mathrm{L}$ TEV protease $(10 \mathrm{mg} / \mathrm{mL})$ and incubated in a dialysis bag in $300 \mathrm{~mL}$ TEV buffer over night at $4{ }^{\circ} \mathrm{C}$.

\section{Buffer exchange and concentration}

The buffer exchange was done with PD10 columns. First the column was equilibrated with $10 \mathrm{~mL}$ coupling buffer, then the protein solution was transferred onto the column and eluted with 3 times $3.5 \mathrm{~L}$ coupling buffer. The protein solution was concentrated with Vivaspin 20 columns to a concentration of $1.14 \mathrm{mg} / \mathrm{mL}$.

\subsubsection{Purification of antibody sera}

Coupling of FMNL-His to CNBr-activated Sepharose 
$1 \mathrm{~g}$ of $\mathrm{CNBr}$-activated sepharose was swollen in $1 \mathrm{mM} \mathrm{HCl}$ for $15 \mathrm{~min}$ and then washed with $200 \mathrm{~mL} 1 \mathrm{M} \mathrm{HCl}$ on a glass filter. The sepharose was then transferred to a Falcon tube with $5 \mathrm{~mL}$ coupling buffer, mixed with the protein solution and incubated over night at $4{ }^{\circ} \mathrm{C}$ under constant shaking. Afterwards the supernatant was removed and some sepharose was tested for protein binding with color reaction of Bradford solution. Then blocking buffer was added to the sepharose and washed for $2 \mathrm{~h}$ at room temperature. The coupled sepharose was then loaded onto an empty column and washed with 5 column volumes of washing buffers 1 and 2 and afterwards washed with 5 column volumes of PBS. The column was stored in PBS $+0.02 \% \mathrm{NaN}_{3}$.

\section{Purification of antibody sera}

The purification was performed at $4{ }^{\circ} \mathrm{C}$. After washing the column with FMNL-His tagged sepharose with PBS (10 column volumes, $1 \mathrm{~mL} / \mathrm{min}$ ) the centrifuged (2x15 $\left.\mathrm{min}, 4^{\circ} \mathrm{C}, 15,000 \mathrm{~g}\right)$ serum was loaded onto the column with a flow rate of $0.5 \mathrm{~mL} / \mathrm{min}$. The flow-through was collected and a sample was taken. The column was washed with 10 column volumes of PBS and 10 column volumes of PBS $+300 \mathrm{mM} \mathrm{NaCl}$ until the OD $\mathrm{A}_{280}$ was stable. The first elution step was done with High Salt Buffer until the OD A280 dropped. The fraction size was $1 \mathrm{~mL}$. Then elution with low $\mathrm{pH}$ and high $\mathrm{pH}$ buffer followed. For elution with high and low $\mathrm{pH}$ buffers, $100 \mu \mathrm{L}$ neutralization buffer was added to the collection tubes before elution. After purification, the fractions were pooled, the buffer was changed to PBS as explained before and the solution was concentrated to a concentration of $1 \mathrm{mg} / \mathrm{mL}$.

\subsubsection{Fly-related methods}

Fly genetics and generation of germ line clones

The $d i a^{s y 5}$ allele carries a point mutation leading to the exchange of a Serin by a Leucin at position 92 of the protein (Yan et al., 2013). The genetic region is flanked by a flipase recognition target (FRT) site on the left chromosome. For the generation of embryos carrying the maternal mutation, dia ${ }^{s y 5}$ FRT were crossed to $O v O^{F R T 2 L}$ flies. These flies carry a Flipase that is expressed upon heat shock, the ovo mutation, leading to female sterility, and the corresponding FRT site. Upon heat shock, the Flipase leads to induction of mitotic recombination that results in female flies with homozygous mutant germline cells (Chou and Perrimon, 1992). Flies in which the mitotic recombination did not happen carry the ovo mutation in their germline leading to sterility (Chou et al., 1993). 
In the same way germ line clones of dizzy, canoe, ELMO, Rap1 and scribble were generated with corresponding $O v 0^{F R T 2 L}$ or $O v O^{F R T 2 R}$ flies.

The dizzy ${ }^{\Delta 8}$ allele was generated by imprecise excision of a P-element, leading to a removal of the transcription start site, the first exon and nearly the full first intron (Huelsmann et al., 2006). The canoeR2 allele, that was generated in an EMS screen has a stop codon at position 211 directly after the first Ras association domain, possibly representing a null-allele (Sawyer et al., 2009). Also, the $E L M O^{367}$ allele was generated in an EMS screen (Luschnig et al., 2004) and was mapped and characterized by Dr. Zhiyi Lv (Winkler et al., 2015). Although no mutation in the genomic region of ELMO could be detected, the lethality of this allele could be mapped to ELMO by a complementation assay with deficiencies that are available from the Bloomington Drosophila stock center. Furthermore, the lethality of the allele can be rescued by endogenous expression of an ELMO-GFP. The Rap $1^{\text {P579 }}$ allele was made by insertion of a Pelement, leading to phenotypes also described for other Rap1 alleles (Huelsmann et al., 2006; Knox and Brown, 2002). The scribble ${ }^{1}$ allele was also generated in a EMS screen, exchanging the Leucine at position 266 to a glutamine (Zeitler et al., 2004).

For the analysis of dia mutant ovaries overexpressing Bazooka GFP, dia ${ }^{s y 5}$ flies with a maternal tub-Gal4 driver and UASp-Bazooka-GFP were crossed to flies expressing hs-Flipase and nls-GFP with a flanking FRT site. After induction of mitotic recombination by heat shock mutant ovaries were marked by missing nuclear GFP-signal.

The generation of germ line clones was done according to Chou and Perrimon, 1992. Heat shocks were done at two consecutive days at first and second larval stages at $37^{\circ} \mathrm{C}$ for one hour to induce expression of the Flipase.

To generate embryos maternally mutant for sponge, I made use of the allele sponge $e^{[242]}$, that was generated in an EMS screen and carries a stop codon (W487STOP) (Biersmith et al., 2011; Postner et al., 1992). These flies were crossed to flies carrying a deficiency of the sponge locus (DF(3R)3450) resulting in viable flies homozygous mutant for sponge giving rise to embryos that died latest during gastrulation (Biersmith et al., 2011; Postner et al., 1992). For GLCs of slam I made use of a fly stock in which the genomic rescue of the $5^{\prime}$ region of $\mathrm{Df}(2 \mathrm{~L})$ slam containing CG9505 was inserted into position 58A3 and recombined with Df(2L) slam FRT2L. 
Expression of constructs driven by UAS-Gal4 system was done with a maternal tubulin-Gal-4 driver according to Brand and Perrimon, 1993. Expression of Bazooka RNAi was done with a triple maternal driver (MTDGal4) (Petrella et al., 2007).

\section{Generation of transgenic flies}

For $\varphi$ C31 mediated transgenesis of attPB3VK00033 flies (Markstein et al., 2008), staged preblastoderm embryos from the fly stock nanos $\varphi 31$ were collected, dechorionated and lined up on pieces of apple juice agar. The embryos are then glued with heptan based glue to a cover glass, dried for $\sim 10$ min in a desiccation chamber, covered with halocarbon oil. The fosmid was injected with a concentration of $250 \mathrm{ng} / \mu \mathrm{L}$ in water. The slides were then incubated at $18{ }^{\circ} \mathrm{C}$ and hatched larvae were collected into food vials and further incubated at room temperature. Adults were crossed to $\mathrm{w}^{-}$flies and the next generation was screened for dsRed expression in the eyes. Males which showed dsRed expression were crossed to TM3/TM6B females and then homozygous possible FMNL-GFP stocks were established and tested for GFP expression.

\section{Fixation of embryos for staining}

Fixation of embryos was done according to Großhans et al., 2005. If stained for Armadillo, heat fixation was performed. For staining of F-actin by Phalloidin or Cpa, a concentration of $8 \%$ formaldehyde was used instead of $4 \%$ and the vitelline membrane was removed by hand. Hand peeling was done by washing the fixation solution off with PBS and the embryos were stuck to double sided tape and covered with PBT. The vitelline membrane was removed with an injection needle. Afterwards the embryos were stored in PBT at $4{ }^{\circ} \mathrm{C}$ for short term before staining.

Preparation of ovaries, testis and larval brains

Preparation of ovaries and testis were done by opening the abdomen of adult male or female flies and removing the tissues from other body parts. Tissue preparation was performed in cold PBS and tissues were stored on ice before fixation. Fixation was done in $9.5^{\circ} \mathrm{mL}$ PBS with $0.5^{\circ} \mathrm{mL}$ Formaldehyde by shaking for $20 \mathrm{~min}$ at room temperature. The tissues were then washed with PBT and stained as described below. 
Immunostaining was performed according to Großhans et al., 2005.

Viability Assay

For viability assays wild type and P/Def embryos were collected on apple juice agar plates $\mathrm{o} / \mathrm{n}$, counted and transferred to fresh apple juice agar plates. Hatched larvae were counted and transferred to food vials in which pupae are counted as well as the hatched adult flies. The number of used embryos, larvae or pupae was counted as $100 \%$.

Injection of $\alpha$-amanitin

$\alpha$-amanitin was injected into embryos expressing CanoeYFP during preblastoderm stage. The embryos were dechorionated, lined up on apple juice agar plates and glued onto cover slides. The drug was injected with a concentration of $1 \mathrm{mg} / \mathrm{mL}$ in water. Afterwards, the embryos were kept at $25^{\circ} \mathrm{C}$ for around 1.5-2 $\mathrm{h}$ to reach late syncytial blastoderm stage. The embryos were then washed from the slides with heptane and fixed, hand peeled and stained as described above.

\subsubsection{Whole mount in situ hybridization}

Production of a probe detecting FMNL

In situ hybridization was done using standard methods described before (Lehmann and Tautz, 1994). To produce the FMNL probe, FMNL cDNA vector was linearized with NotI. The sample of $50 \mu \mathrm{L}$ was mixed with $200 \mu \mathrm{L}$ phenolchloroform mix, centrifuged with maximum speed for $5 \mathrm{~min}$ at $4{ }^{\circ} \mathrm{C}$ and the upper aqueous phase was transferred to a new Eppendorf tube and $200 \mu \mathrm{L}$ Chloroform were added. After centrifugation with maximum speed for $5 \mathrm{~min}$ at $4{ }^{\circ} \mathrm{C}$, the upper phase was again transferred to a new tube and DNA was precipitated by adding $20 \mu \mathrm{L} \mathrm{NaAc}$ and $500 \mu \mathrm{L}$ ethanol and incubation for $30 \mathrm{~min}$ at $-80^{\circ} \mathrm{C}$. After centrifugation for $30 \mathrm{~min}$ with maximum speed at $4{ }^{\circ} \mathrm{C}$, the pellet was washed with $70 \%$ ethanol, dried and solved in $10 \mu \mathrm{L}$ RNasefree water. The linearized cDNA was then transcribed with T7 RNA polymerase using Dig-NTPs to label the probe as followed:

$\begin{array}{ll}\text { Linearized DNA }(1 \mu \mathrm{g}) & 3 \mu \mathrm{L} \\ \text { 10x NTP-Dig Labeling Mix } & 2 \mu \mathrm{L} \\ \text { 10x Transcription buffer } & 2 \mu \mathrm{L} \\ \text { RNA Polymerase T7 } & 2 \mu \mathrm{L}\end{array}$


RNase inhibitor

RNase free water
$1 \mu \mathrm{L}$

Adjust to $20 \mu \mathrm{L}$

The mix was incubated at $37^{\circ} \mathrm{C}$ for $2 \mathrm{~h}$ in a PCR machine. Afterwards $0.8 \mu \mathrm{L}$ of $0.5 \mathrm{M}$ EDTA, $2 \mu \mathrm{L}$ of $5 \mathrm{M} \mathrm{LiCl}$ and $75 \mu \mathrm{L}$ cold ethanol were added and incubated for at least $30 \mathrm{~min}$ at $4{ }^{\circ} \mathrm{C}$. After incubation the mix was centrifuged with maximum speed for $10 \mathrm{~min}$ at $4{ }^{\circ} \mathrm{C}$ and the pellet was washed twice with $70 \%$ cold ethanol. The pellet was dissolved in $20 \mu \mathrm{L}$ DEPC-treated water and the probe was stored at $-20^{\circ} \mathrm{C}$. To produce a working solution $2 \mu \mathrm{L}$ of the probe was mixed with $1 \mu \mathrm{L}$ tRNA $(50 \mathrm{mg} / \mathrm{mL})$ and $20 \mu \mathrm{L}$ DEPC-treated water and boiled for $4 \mathrm{~min}$ at $100{ }^{\circ} \mathrm{C}$. Afterwards the probe was immediately cooled down in ice water and mixed with $200 \mu \mathrm{L}$ hybridization solution. The slam probe was prepared by Dr. Shuling Yan.

\section{Hybridization and detection of RNA}

For hybridization FA-fixed embryos were transferred to PBT, washed twice for $5 \mathrm{~min}$, then washed for $10 \mathrm{~min}$ in hybridization solution:PBT-mix (1:1) and then incubated with hybridization solution for $10 \mathrm{~min}$ at room temperature. Prehybridization with hybridization solution was done at $57^{\circ} \mathrm{C}$ in a water bath for $1 \mathrm{~h}$. Afterwards embryos were incubated with the diluted probe (1:10) in hybridization solution overnight at $57^{\circ} \mathrm{C}$ and then washed with decreasing concentrations of hybridization solution in PBT.

To detect the probe, an antibody against DIG labeled with alkaline phosphatase (AP) was used as described before for immunostainings. After incubation with the antibody, the embryos were transferred into AP buffer and washed trice for $5 \mathrm{~min}$. To induce color reaction $4.5 \mu \mathrm{L}$ NBT and $3.5 \mu \mathrm{L}$ BCIP were added to embryos in $1 \mathrm{~mL}$ AP buffer. The enzymatic color reaction was detected after 5-10 min and stopped by washing with PBT. To remove background, the embryos were washed in a series of increasing ethanol in PBT to $100 \%$ ethanol and back to $100 \%$ PBT. The embryos were mounted in Aquapolymount and imaged with a light microscope.

\subsubsection{Imaging \\ Imaging of fixed samples}

Fixed and immunostained embryos were imaged at the LSM microscope with $63 x$ water objectives. Larval tissues were imaged with $25 x$ and $40 x$ objectives. The laser intensities were set to optimal illumination. Top views of embryos 
were made with a $2 x$ zoom and images had a size of $67.5 \times 67.5 \mu \mathrm{m}$ (512 x512 pixel) and a lateral pixel size of $130 \mathrm{~nm}$. Side views were imaged with a zoom of $1.4 x$ and had a size of $96.4 \times 29.4 \mu \mathrm{m}(512 \times 200$ pixel $)$ and a lateral pixel size of $190 \mathrm{~nm}$. Z-stacks were made with a step size of $0.5 \mu \mathrm{m}$. Each channel was recorded separately. Images of phalloidin staining in wild type and dia embryos were obtained with a size of $67.48 \times 67.48 \mu \mathrm{m}$ (512 x 512 pixel) and a lateral pixel size of $131.8 \mathrm{~nm}$. Z-stacks with a step size of $0.5 \mu \mathrm{m}$ were made with same laser intensities for both phenotypes which were mounted of the same slide and distinguished by a Myo-GFP signal that was only expressed by dia mutants.

\section{Live imaging}

For live imaging embryos were handled as described before (Kanesaki et al., 2011). Live imaging of embryos expressing CanoeYFP and CherrySlam were gained with a frame size of $28.7 \times 28.7 \mu \mathrm{m}(256 \times 256$ pixel $)$ and a lateral pixel size of $110 \mathrm{~nm}$ in an interval of $60 \mathrm{~s}$. Each channel was recorded separately and channels were changed after recording of every z-stack. Z-stacks had a size of 19 slices with a step size of $0.5 \mu \mathrm{m}$. Embryos expressing ScribbledGFP were imaged with AiryScan detection with a frame size of $36 \times 36 \mu \mathrm{m}$ (488 x 488 pixel) and a lateral pixel size of $73 \mathrm{~nm}$. Z-stacks were obtained in an interval of $60 \mathrm{~s}$ and every z-stack included 11 slices with a step size of $1 \mu \mathrm{m}$. Embryos expressing ELMO-GFP and CherrySlam were imaged with AiryScan detection with a frame size of $32 \times 32 \mu \mathrm{m}(488 \times 488$ pixel $)$ and a lateral pixel size of $66 \mathrm{~nm}$. Z-stacks were obtained in an interval of $60 \mathrm{~s}$ and every z-stack contained 17 slices with a step size of $0.5 \mu \mathrm{m}$. Images of embryos expressing GFP-Rap1 were obtained with AiryScan detection with a frame size of $32.1 \times 32.1 \mu \mathrm{m}(476 \times 476$ pixel $)$ with a lateral pixel size of $67.5 \mathrm{~nm}$. Z-stacks were obtained with a step size of $0.2 \mu \mathrm{m}$ and orthogonal views were conducted with Fiji/ImageJ. Images of living embryos expressing the Rhosensor-tdtomato was done with AiryScan. The frame size was $32.16 \times 32.16 \mu \mathrm{m}(488 \times 488$ pixel) with a lateral pixel size of $66 \mathrm{~nm}$. Z-stacks were recorded from apical to basal end of the furrow with a step size of $0.4 \mu \mathrm{m}$ and orthogonal views were obtained using Fiji/ ImageJ. Top views of embryos expressing BazookaGFP were recorded with AiryScan detection and a frame size of $32.16 \times 32.16 \mu \mathrm{m}(488 \times 488$ pixel $)$ with a lateral pixel size of $66 \mathrm{~nm}$. Every minute a z-stack with 22 slices, covering $11 \mu \mathrm{m}$ was imaged. For Figure 23 slices covering $8.5 \mu \mathrm{m}$ were summed using Fiji/ ImageJ. 
Fluorescence recovery after photobleaching (FRAP)

FRAP experiments were performed at the LSM microscope. For bleaching of CanoeYFP, the laser intensity was set to $50 \%$ and bleaching was done with 100 iterations in fast FRAP mode. Imaging was started directly after bleaching and was done with z-stacks ( 3 slices, step size of $1.5 \mu \mathrm{m}$ ) in an interval of $30 \mathrm{~s}$. The images were recorded with a frame size of $50 \times 50 \mu \mathrm{m}(512 \times 512$ pixel $)$ with a lateral pixel size of $97.7 \mathrm{~nm}$. The z-stacks were merged for quantification. FRAP of 117-GFP was done with laser power set to $100 \%$ and 150 iterations in fast FRAP mode. Imaging was done with a frame size of $33.7 \times 33.7 \mu \mathrm{m}(512 \times 512$ pixel $)$ and a lateral pixel size of $65.8 \mathrm{~nm}$ and in an interval of $5 \mathrm{~s}$.

\section{Two-Photon microscopy}

Living embryos expressing BazookaGFP either in wild type or dia background were imaged with a two-photon microscope to allow for side views. Images were taken every $10 \mathrm{~min}$ with correction for $\mathrm{z}$ - position. The frame size of each image was $85.02 \times 49.82 \mu \mathrm{m}(512 \times 300$ pixel $)$ with a lateral pixel size of $166 \mathrm{~nm}$.

\subsubsection{Quantifications}

All measurements for quantifications were done in Fiji/ ImageJ and further calculations were done in Microsoft Excel if not stated otherwise.

Fluorescence intensities along cellularization furrows of side views

For quantification of fluorescence intensities along cellularization furrows of fixed embryos segmented lines were drawn along the furrows from apical to basal and fluorescence intensities were measured using the line plot function of Fiji/ ImageJ. The maximal intensities for each furrow were normalized to one and apical - basal positions were normalized to the peak if possible. Averages were calculated after normalization with standard error of the mean as error bars and plotted against the apical basal position. Heat maps were made with the conditional formatting function for each furrow.

Fluorescence intensities along cellularization furrows of top views

For quantification of fluorescence intensities along cellularization furrows of living embryos from top views, the fluorescence intensity of three furrows in one embryo were measured in each z-position along the apical - basal axis for 
the indicated time points. The maximal intensities for each protein were normalized to one, averaged and plotted against the apical - basal position. For the measurement of the width of Canoe signal in Figure 8the distribution of fluorescence intensity at one furrow was measured at different time points using the line plot function. Position zero on the $\mathrm{x}$-axis was defined by the peak of the curve at the latest time point. The width of the Canoe region was measured for Figure 8 was measured at three furrows at time points as indicated and plotted against the time.

FRAP

Quantification of FRAP results was done by measuring the mean fluorescence intensity in the bleached region before and after bleach over time. Furthermore, the mean fluorescence intensity outside of the bleached region was measured. The fluorescence intensities were then normalized to their individual peak and to the outside to correct for bleaching. The averages with SEM were plotted against the time.

Measurement of fluorescence intensities in fixed top views and calculation of exponential decay

Quantification of fluorescence intensities of phalloidin staining in wild type and dia embryos was done by measuring mean fluorescence intensities in four frames with a size of $256 \times 256$ pixel per embryo. As the embryos were costained in the same vial and imaged with same laser intensities, the measured intensities could be plotted for both genotypes without any normalization. Averages of intensities from 3 embryos per genotype with SEM were also plotted.

Measurement of Cpa signal at the edges of caps in wild type and dia embryos were done by drawing a line perpendicular to the edge across the edge. Every measurement was normalized to its peak and the peak was set as position zero. The measurements for every embryo was averaged and plotted with SEM against the position. For the determination of the width of the Cpa signal, the width of the curves for the averages from every measurement was quantified at a relative fluorescence intensity of 0.8 and plotted for both genotypes as box plots with the error bars showing the maximal and minimal measured width. 
For the calculation of the exponential decay, the data points from the averages of every embryo $-1.6 \mu \mathrm{m}$ to $1.6 \mu \mathrm{m}$ were mirrored at position zero and with error bars plotted in Origin Pro 8.5G. For every of the six curves an exponential curve was fitted and the exponential decay was plotted for each genotype together with the average and SEM. 


\section{List of figures}

Figure 1 Cortex and cortical domains................................................................

Figure 2 Dynamics of cortical domains in Drosophila embryos. ...........................

Figure 3 Cortical domains during syncytial blastoderm. ...................................11

Figure 4 Cortical domains during cellular blastoderm/cellularization. ...........15

Figure 5 Dynamics of cortical domains in the Drosophila blastoderm embryo.

Figure 6 CanoeYFP and CherrySlam dynamics during mitosis 13 and

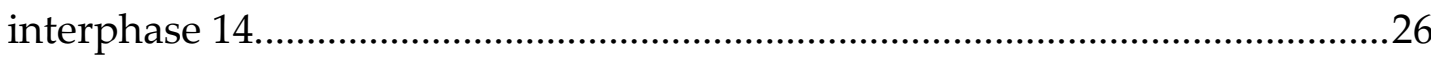

Figure 7 Localization of Canoe and Slam at onset of cellularization................27

Figure 8 Dynamics of Canoe and Slam at onset of cellularization....................28

Figure 9 ScribbleGFP dynamics during mitosis 13 and interphase 14 ............30

Figure 10 Dynamics of ScribbleGFP during early cellularization......................31

Figure 11 Genetic control of subapical Canoe by lateral and basal factors.....32

Figure 12 Dynamics of GFP-Rap1 during cellularization....................................33

Figure 13 Genetic control of subapical Canoe by potential upstream factors.

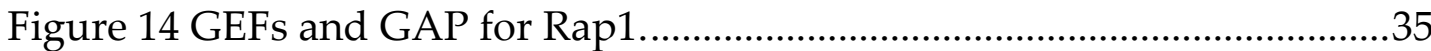

Figure 15 Blastoderm phenotype of embryos from ELMO germline clones. .36

Figure 16 ELMO and Sponge are required for subapical restriction of Canoe.

Figure 17 Sponge localization during early embryonic development...............38

Figure 18 Membrane localization of Sponge is mediated by ELMO. ...............39

Figure 19 Genetic control of subapical Sponge. ..................................................40

Figure 20 ELMO-GFP and CherrySlam dynamics during mitosis 13 and

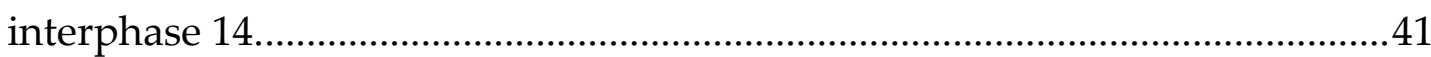

Figure 21 Dynamics of ELMO-GFP during early cellularization. ......................42

Figure 22 Subapical enrichment of Baz during cellularization in fixed embryos.

Figure 23 Subapical enrichment of Baz during cellularization in living

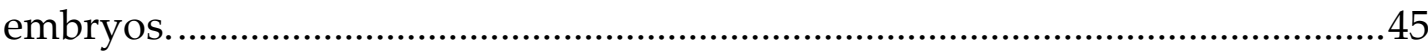

Figure 24 BazookaGFP dynamics imaged by two-photon microscopy............46

Figure 25 ELMO is required for subapical enrichment of Baz and Arm.........47

Figure $26 \mathrm{Baz}$ functions in Cno localization during cellularization ...................48

Figure 27 Spg localization is not affected by Baz...............................................49 
Figure 28 Zygotic gene expression is necessary for Canoe localization during cellularization. 50

Figure 29 Cno localizes to subapical domain at onset of cellularization independently of the cell cycle number. 51 Figure 30 Model for the formation of the subapical domain during onset of cellularization. 56

Figure 31 Scheme of actin dynamics in non-muscle cells. 62

Figure 32 Scheme of domain composition of Drosophila Dia. 63

Figure 33 Genetic region of dia and alleles.... 66

Figure 34 Dia is reduced in dia mutants.

Figure 35 Antagonistic genetic interaction of dia and baz during oogenesis.. 68 Figure 36 Genetic control of subapical Baz and Arm localization................... 70

Figure $37 \mathrm{Dia}$ is required for subapical Arm enrichment. ............................. 71

Figure $38 \mathrm{Dia}$ is required for subapical Baz enrichment. ............................... 73

Figure 39 Dynamics of Baz-GFP during cellularization in wild type and dia

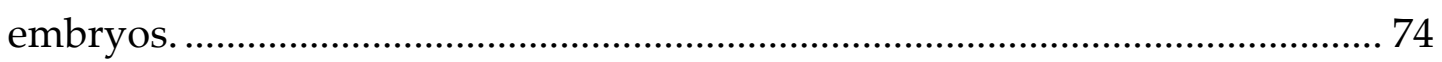

Figure $40 \mathrm{Dia}$ is required for subapical Cno enrichment. ............................... 75

Figure 41 Subapical enrichment of Spg in wild type and dia embryos........... 77

Figure 42 F-actin is enriched at furrow canals during cellularization. ........... 79

Figure $43 \mathrm{Dia}$ is enriched at furrow canals during cellularization. ................. 80

Figure 44 Dynamics of the Rho binding domain. ........................................... 81

Figure 45 Recovery of CanoeYFP after bleaching in wild type and dia during cellularization.

Figure 46 Recovery of the integral membrane protein 117GFP in wild type and dia preblastoderm embryos. 84

Figure 47 Subapical enrichment of Baz is not perturbed in shi embryos........ 85

Figure 48 yRab35 localizes to all cortical domains during cellularization. .... 87

Figure 49 Localization of yRab23 during early embryogenesis...................... 88

Figure 50 Dia localization to actin cap rims in syncytial blastoderm wild type embryos.

Figure 51 Cpa enrichment at the rims of actin caps during syncytial blastoderm in wild type embryos.

Figure 52 The separation of cap and intercap domains in wild type and dia embryos.

Figure 53 The Cpa signal at the rim of actin caps gets broader in dia mutants. 
Figure 54 Cpa enrichment at cap edges in wild type and dia syncytial

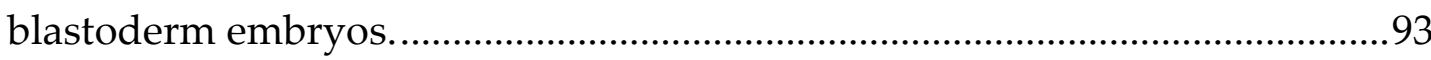

Figure 55 Cpa enrichment at cap rims in wild type and dia............................94

Figure 56 Fluorescence intensity of F-actin in wild type and dia syncytial blastoderm embryos.

Figure $57 \mathrm{Cpa}$ and F-actin enrichment at actin caps and basal tips in wild type and dia during early cellularization.

Figure 58 Influence of a polarized actin cortex on the formation of a cortical domain 101

Figure 59 Domain structure of FMNL. ...........................................................107

Figure 60 Genetic map of FMNL-deficiency.................................................108

Figure 61 Genetic map of P-Element insertion into the FMNL locus. ...........109

Figure 62 Quantitative real time PCR of p/Def and wild type embryonic extracts .110

Figure 63 Viability assay of wild type and P/Def flies. .111

Figure $64 \mathrm{P} /$ Def flies show reduced negative geotaxis. .112

Figure 65 Antibodies against FMNL show no specific signal in wild type embryos.

Figure 66 Purification of FMNL-Fragment with N-terminal ZZ- and Cterminal His-tag.

Figure 67 ZZ-Tag cleavage with TEV protease and further purification of FMNL-His fragment.

Figure 68 Western blot of wild type embryonic extracts against FMNL.......116

Figure 69 FMNL is expressed during embryonic development...................116

Figure 70 Map of the fosmid used for the generation of FMNL-GFP flies. ..117

Figure 71 FMNL-GFP is expressed during cellularization. 118

Figure 72 FMNL-GFP is expressed in testis and ovary 120

Figure 73 FMNL-GFP shows strong expression in larval brains. 121

Figure 74 FMNL-GFP is enriched in glia cells in larval brains. 122

Figure 75 FMNL-GFP is expressed in adult heads. 123 


\section{List of tables}

Table 1 Buffers and solutions 129

Table 2 Oligunucleotides used in this study .................................................. 131

Table 3 Plasmids and fosmids used in this study....................................... 132

Table 4 Primary antibodies used in this study............................................... 132

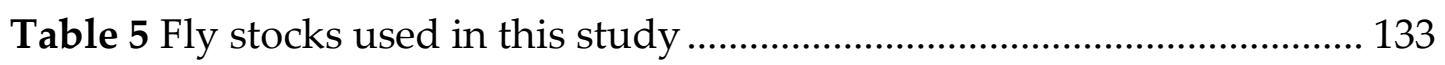

Table 6 Normalized values of fluorescence intensities measured for Figure 8B.

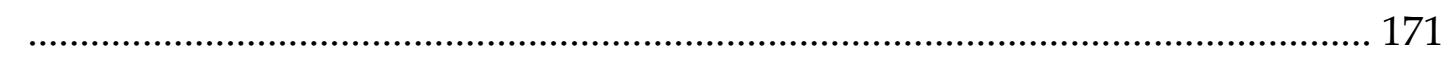

Table 7 Normalized fluorescence intensity measured for Figure 8C ........... 173

Table 8 Furrow width marked by Canoe measured for Figure 8D .............. 174

Table 9 Normalized fluorescence intensity of Scrib signal measured for Figure 10C.

Table 10 Normalized fluorescence intensity of Canoe signal measured for

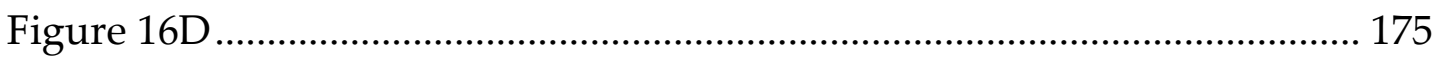

Table 11 Normalized fluorescence intensities of ELMO-GFP and CherrySlam

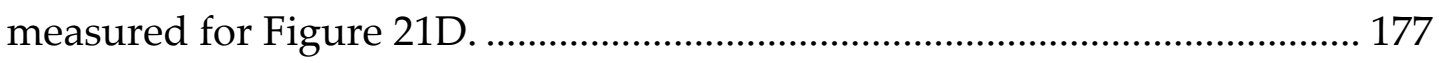

Table 12 Normalized fluorescence intensities of Armadillo signal for Figure $37 \mathrm{C}$ 178

Table 13 Normalized fluorescence intensities of Baz signal measured for Figure 38C

Table 14 Normalized fluorescence intensity of Cno signal measured for Figure $40 \mathrm{C}$ 188

Table 15 Normalized fluorescence intensities of Spg singal measured for Figure 41C. 191

Table 16 Normalized fluorescence intensities of CnoYFP signal measured for Figure 45C. 196

Table 17 Normalized fluorescence intensities of 117GFP signal measured for Figure 46B. 196

Table 18 Averages of normalized fluorescence intensities per embryo measured for Figure 54B................................................................................. 198

Table 19 Width of Cpa signal curves measured for Figure 54C.................... 199

Table 20 Exponential decay of Cpa curves fitted for Figure 55C.................. 200

Table 21 Fluorescence intensities of Phalloidin measured for Figure 56C... 200

Table 22 Distance of flies measured in negative geotaxis assay for Figure 64. 


\section{Bibliography}

Abreu-Blanco, M. T., Verboon, J. M. and Parkhurst, S. M. (2011). Cell wound repair in Drosophila occurs through three distinct phases of membrane and cytoskeletal remodeling. J. Cell Biol. 193, 455-464.

Abreu-Blanco, M. T., Verboon, J. M. and Parkhurst, S. M. (2014). Coordination of Rho Family GTPase Activities to Orchestrate Cytoskeleton Responses during Cell Wound Repair. Curr. Biol. 24, 144155.

Acharya, S., Laupsien, P., Wenzl, C., Yan, S. and Großhans, J. (2014). Function and dynamics of slam in furrow formation in early Drosophila embryo. Dev. Biol. 386, 371-384.

Afshar, K., Stuart, B. and Wasserman, S. A. (2000). Functional analysis of the Drosophila diaphanous FH protein in early embryonic development. Development 127, 1887-1897.

Amândio, A. R., Gaspar, P., Whited, J. L. and Janody, F. (2014). Subunits of the Drosophila Actin-Capping Protein Heterodimer Regulate Each Other at Multiple Levels. PLOS ONE 9, e96326.

Benton, R. and Johnston, D. S. (2003). Drosophila PAR-1 and 14-3-3 Inhibit Bazooka/PAR-3 to Establish Complementary Cortical Domains in Polarized Cells. Cell 115, 691-704.

Benzer, S. (1967). BEHAVIORAL MUTANTS OF Drosophila ISOLATED BY COUNTERCURRENT DISTRIBUTION. Proc. Natl. Acad. Sci. U. S. A. 58, 1112-1119.

Biersmith, B., Liu, Z., Bauman, K. and Geisbrecht, E. R. (2011). The DOCK Protein Sponge Binds to ELMO and Functions in Drosophila Embryonic CNS Development. PLOS ONE 6, e16120.

Biersmith, B., Wang, Z.-H. and Geisbrecht, E. R. (2015). Fine-Tuning of the Actin Cytoskeleton and Cell Adhesion During Drosophila Development by the Unconventional Guanine Nucleotide Exchange Factors Myoblast City and Sponge. Genetics 200, 551-567.

Bilder, D. and Perrimon, N. (2000). Localization of apical epithelial determinants by the basolateral PDZ protein Scribble. Nature 403, 676680.

Bilder, D., Li, M. and Perrimon, N. (2000). Cooperative regulation of cell polarity and growth by Drosophila tumor suppressors. Science 289, 113116.

Bilder, D., Schober, M. and Perrimon, N. (2003). Integrated activity of PDZ protein complexes regulates epithelial polarity. Nat. Cell Biol. 5, 53-58.

Blankenship, J. T., Backovic, S. T., Sanny, J. S. P., Weitz, O. and Zallen, J. A. (2006). Multicellular Rosette Formation Links Planar Cell Polarity to Tissue Morphogenesis. Dev. Cell 11, 459-470.

Block, J., Breitsprecher, D., Kühn, S., Winterhoff, M., Kage, F., Geffers, R., Duwe, P., Rohn, J. L., Baum, B., Brakebusch, C., et al. (2012). FMNL2 
drives actin-based protrusion and migration downstream of Cdc42. Curr. Biol. CB 22, 1005-1012.

Blythe, S. A. and Wieschaus, E. F. (2015). Coordinating Cell Cycle Remodeling with Transcriptional Activation at the Drosophila MBT. Curr. Top. Dev. Biol. 113, 113-148.

Boettner, B., Govek, E.-E., Cross, J. and Aelst, L. V. (2000). The junctional multidomain protein AF-6 is a binding partner of the Rap1A GTPase and associates with the actin cytoskeletal regulator profilin. Proc. Natl. Acad. Sci. 97, 9064-9069.

Boettner, B., Harjes, P., Ishimaru, S., Heke, M., Fan, H. Q., Qin, Y., Aelst, L. V. and Gaul, U. (2003). The AF-6 Homolog Canoe Acts as a Rap1 Effector During Dorsal Closure of the Drosophila Embryo. Genetics 165, 159-169.

Bogdan, S., Schultz, J. and Grosshans, J. (2013). Formin' cellular structures. Commun. Integr. Biol. 6, e27634.

Bombardier, J. P., Eskin, J. A., Jaiswal, R., Corrêa, I. R., Xu, M.-Q., Goode, B. L. and Gelles, J. (2015). Single-molecule visualization of a formincapping protein 'decision complex" at the actin filament barbed end.' Nat. Commun. 6,.

Bonello, T. T., Perez-Vale, K. Z., Sumigray, K. D. and Peifer, M. (2018). Rap1 acts via multiple mechanisms to position Canoe and adherens junctions and mediate apical-basal polarity establishment. Development 145, dev157941.

Bos, J. L. (2006). Epac proteins: multi-purpose cAMP targets. Trends Biochem. Sci. 31, 680-686.

Brand, A. H. and Perrimon, N. (1993). Targeted gene expression as a means of altering cell fates and generating dominant phenotypes. Development 118, 401-415.

Buszczak, M., Paterno, S., Lighthouse, D., Bachman, J., Planck, J., Owen, S., Skora, A. D., Nystul, T. G., Ohlstein, B., Allen, A., et al. (2007). The Carnegie Protein Trap Library: A Versatile Tool for Drosophila Developmental Studies. Genetics 175, 1505-1531.

Campanale, J. P., Sun, T. Y. and Montell, D. J. (2017). Development and dynamics of cell polarity at a glance. J Cell Sci 130, 1201-1207.

Cao, J., Albertson, R., Riggs, B., Field, C. M. and Sullivan, W. (2008). Nuf, a Rab11 effector, maintains cytokinetic furrow integrity by promoting local actin polymerization. J. Cell Biol. 182, 301-313.

Castrillon, D. H. and Wasserman, S. A. (1994). Diaphanous is required for cytokinesis in Drosophila and shares domains of similarity with the products of the limb deformity gene. Development 120, 3367-3377.

Cherfils, J. and Zeghouf, M. (2013). Regulation of Small GTPases by GEFs, GAPs, and GDIs. Physiol. Rev. 93, 269-309. 
Choi, W., Harris, N. J., Sumigray, K. D. and Peifer, M. (2013). Rap1 and Canoe/afadin are essential for establishment of apical-basal polarity in the Drosophila embryo. Mol. Biol. Cell 24, 945-963.

Chou, T. B. and Perrimon, N. (1992). Use of a Yeast Site-Specific Recombinase to Produce Female Germline Chimeras in Drosophila. Genetics 131, 643653.

Chou, T. B., Noll, E. and Perrimon, N. (1993). Autosomal P[ovoD1] dominant female-sterile insertions in Drosophila and their use in generating germ-line chimeras. Development 119, 1359-1369.

Coopman, P. and Djiane, A. (2016). Adherens Junction and E-Cadherin complex regulation by epithelial polarity. Cell. Mol. Life Sci. 73, 35353553.

Coravos, J. S. and Martin, A. C. (2016). Apical Sarcomere-like Actomyosin Contracts Nonmuscle Drosophila Epithelial Cells. Dev. Cell 39, 346-358.

Côté, J.-F., Motoyama, A. B., Bush, J. A. and Vuori, K. (2005). A novel and evolutionarily conserved PtdIns(3,4,5)P3-binding domain is necessary for DOCK180 signaling. Nat. Cell Biol. 7, 797-807.

Cytrynbaum, E. N., Sommi, P., Brust-Mascher, I., Scholey, J. M. and Mogilner, A. (2005). Early Spindle Assembly in Drosophila Embryos: Role of a Force Balance Involving Cytoskeletal Dynamics and Nuclear Mechanics. Mol. Biol. Cell 16, 4967-4981.

DeBiasio, R. L., LaRocca, G. M., Post, P. L. and Taylor, D. L. (1996). Myosin II transport, organization, and phosphorylation: evidence for cortical flow/solation-contraction coupling during cytokinesis and cell locomotion. Mol. Biol. Cell 7, 1259-1282.

Dollar, G., Gombos, R., Barnett, A. A., Sanchez Hernandez, D., Maung, S. M. T., Mihály, J. and Jenny, A. (2016). Unique and Overlapping Functions of Formins Frl and DAAM During Ommatidial Rotation and Neuronal Development in Drosophila. Genetics 202, 1135-1151.

Dunst, S., Kazimiers, T., von Zadow, F., Jambor, H., Sagner, A., Brankatschk, B., Mahmoud, A., Spannl, S., Tomancak, P., Eaton, S., et al. (2015). Endogenously Tagged Rab Proteins: A Resource to Study Membrane Trafficking in Drosophila. Dev. Cell 33, 351-365.

Edgar, B. A., Kiehle, C. P. and Schubiger, G. (1986). Cell cycle control by the nucleo-cytoplasmic ratio in early Drosophila development. Cell 44, 365372.

Efetova, M., Petereit, L., Rosiewicz, K., Overend, G., Haußig, F., Hovemann, B. T., Cabrero, P., Dow, J. A. T. and Schwärzel, M. (2013). Separate roles of PKA and EPAC in renal function unraveled by the optogenetic control of cAMP levels in vivo. J Cell Sci 126, 778-788.

Eguchi, K., Yoshioka, Y., Yoshida, H., Morishita, K., Miyata, S., Hiai, H. and Yamaguchi, M. (2013). The Drosophila DOCK family protein sponge is involved in differentiation of R7 photoreceptor cells. Exp. Cell Res. 319, 2179-2195. 
Ejsmont, R. K., Sarov, M., Winkler, S., Lipinski, K. A. and Tomancak, P. (2009). A toolkit for high-throughput, cross-species gene engineering in Drosophila. Nat. Methods 6, 435-437.

Emmons, S., Phan, H., Calley, J., Chen, W., James, B. and Manseau, L. (1995). Cappuccino, a Drosophila maternal effect gene required for polarity of the egg and embryo, is related to the vertebrate limb deformity locus. Genes Dev. 9, 2482-2494.

Fabrowski, P., Necakov, A. S., Mumbauer, S., Loeser, E., Reversi, A., Streichan, S., Briggs, J. A. G. and De Renzis, S. (2013). Tubular endocytosis drives remodelling of the apical surface during epithelial morphogenesis in Drosophila. Nat. Commun. 4, 2244.

Farrell, J. A. and O'Farrell, P. H. (2014). From egg to gastrula: How the cell cycle is remodeled during the Drosophila mid-blastula transition. Annu. Rev. Genet. 48, 269-294.

Field, C. M. and Alberts, B. M. (1995). Anillin, a contractile ring protein that cycles from the nucleus to the cell cortex. J. Cell Biol. 131, 165-178.

Foe, V. E. and Alberts, B. M. (1983). Studies of nuclear and cytoplasmic behaviour during the five mitotic cycles that precede gastrulation in Drosophila embryogenesis. J. Cell Sci. 61, 31-70.

Foe, V. E., Odell, G. M. and Edgar, B. A. (1993). Mitosis and morphogenesis in the Drosophila embryo: Point and counterpoint. In The Development of Drosophila melanogaster, M. Bate and A. Martinez Arias, pp. 149-300. Cold Spring Harbor Laboratory.

Frescas, D., Mavrakis, M., Lorenz, H., Delotto, R. and Lippincott-Schwartz, J. (2006). The secretory membrane system in the Drosophila syncytial blastoderm embryo exists as functionally compartmentalized units around individual nuclei. J. Cell Biol. 173, 219-230.

Fricke, R., Gohl, C., Dharmalingam, E., Grevelhörster, A., Zahedi, B., Harden, N., Kessels, M., Qualmann, B. and Bogdan, S. (2009). Drosophila Cip4/Toca-1 Integrates Membrane Trafficking and Actin Dynamics through WASP and SCAR/WAVE. Curr. Biol. 19, 1429-1437.

Gassama-Diagne, A. and Payrastre, B. (2009). Phosphoinositide signaling pathways: promising role as builders of epithelial cell polarity. Int. Rev. Cell Mol. Biol. 273, 313-343.

Gay, N. J. and Keith, F. J. (1992). Regulation of translation and proteolysis during the development of embryonic dorso-ventral polarity in Drosophila. Biochim. Biophys. Acta BBA - Gene Struct. Expr. 1132, 290296.

Goldstein, B. and Macara, I. G. (2007). The PAR Proteins: Fundamental Players in Animal Cell Polarization. Dev. Cell 13, 609-622.

Green, M. R., Sambrook, J. and Sambrook, J. (2012). Molecular cloning: a laboratory manual. 4th ed. Cold Spring Harbor, N.Y: Cold Spring Harbor Laboratory Press. 
Grikscheit, K., Frank, T., Wang, Y. and Grosse, R. (2015). Junctional actin assembly is mediated by Formin-like 2 downstream of Rac1. J Cell Biol 209, 367-376.

Großhans, J., Wenzl, C., Herz, H.-M., Bartoszewski, S., Schnorrer, F., Vogt, N., Schwarz, H. and Müller, H.-A. (2005). RhoGEF2 and the formin Dia control the formation of the furrow canal by directed actin assembly during Drosophila cellularisation. Development 132, 1009-1020.

Harris, T. J. C. (2012). Adherens Junction Assembly and Function in the Drosophila Embryo. Int. Rev. Cell Mol. Biol. 293, 45-83.

Harris, T. J. C. and Peifer, M. (2004). Adherens junction-dependent and independent steps in the establishment of epithelial cell polarity in Drosophila. J. Cell Biol. 167, 135-147.

Harris, T. J. C. and Peifer, M. (2005). The positioning and segregation of apical cues during epithelial polarity establishment in Drosophila. J Cell Biol 170, 813-823.

He, B., Martin, A. and Wieschaus, E. (2016). Flow-dependent myosin recruitment during Drosophila cellularization requires zygotic dunk activity. Dev. Camb. Engl. 143, 2417-2430.

Higashida, C., Miyoshi, T., Fujita, A., Oceguera-Yanez, F., Monypenny, J., Andou, Y., Narumiya, S. and Watanabe, N. (2004). Actin Polymerization-Driven Molecular Movement of mDia1 in Living Cells. Science 303, 2007-2010.

Homem, C. C. F. and Peifer, M. (2008). Diaphanous regulates myosin and adherens junctions to control cell contractility and protrusive behavior during morphogenesis. Development 135, 1005-1018.

Honigmann, A. and Pralle, A. (2016). Compartmentalization of the Cell Membrane. J. Mol. Biol. 428, 4739-4748.

Huelsmann, S., Hepper, C., Marchese, D., Knöll, C. and Reuter, R. (2006). The PDZ-GEF Dizzy regulates cell shape of migrating macrophages via Rap1 and integrins in the Drosophila embryo. Development 133, 29152924.

Hunter, C. and Wieschaus, E. (2000). Regulated Expression of nullo Is Required for the Formation of Distinct Apical and Basal Adherens Junctions in the Drosophila Blastoderm. J. Cell Biol. 150, 391-402.

Hutterer, A., Betschinger, J., Petronczki, M. and Knoblich, J. A. (2004). Sequential roles of Cdc42, Par-6, aPKC, and Lgl in the establishment of epithelial polarity during Drosophila embryogenesis. Dev. Cell 6, 845854.

Insolera, R., Chen, S. and Shi, S.-H. (2011). Par proteins and neuronal polarity. Dev. Neurobiol. 71, 483-494.

Isenberg, G., Aebi, U. and Pollard, T. D. (1980). An actin-binding protein from Acanthamoeba regulates actin filament polymerization and interactions. Nature 288, 455-459. 
Isogai, T., Kammen, R. van der and Innocenti, M. (2015). SMIFH2 has effects on Formins and p53 that perturb the cell cytoskeleton. Sci. Rep. 5, 9802.

Jewett, C. E., Vanderleest, T. E., Miao, H., Xie, Y., Madhu, R., Loerke, D. and Blankenship, J. T. (2017). Planar polarized Rab35 functions as an oscillatory ratchet during cell intercalation in the Drosophila epithelium. Nat. Commun. 8, 476.

Kage, F., Winterhoff, M., Dimchev, V., Mueller, J., Thalheim, T., Freise, A., Brühmann, S., Kollasser, J., Block, J., Dimchev, G., et al. (2017a). FMNL formins boost lamellipodial force generation. Nat. Commun. 8,.

Kage, F., Steffen, A., Ellinger, A., Ranftler, C., Gehre, C., Brakebusch, C., Pavelka, M., Stradal, T. and Rottner, K. (2017b). FMNL2 and -3 regulate Golgi architecture and anterograde transport downstream of Cdc42. Sci. Rep. 7,.

Kanesaki, T., Edwards, C. M., Schwarz, U. S. and Grosshans, J. (2011). Dynamic ordering of nuclei in syncytial embryos: a quantitative analysis of the role of cytoskeletal networks. Integr. Biol. Quant. Biosci. Nano Macro 3, 1112-1119.

Karr, T. L. and Alberts, B. M. (1986). Organization of the cytoskeleton in early Drosophila embryos. J. Cell Biol. 102, 1494-1509.

Kellogg, D. R., Mitchison, T. J. and Alberts, B. M. (1988). Behaviour of microtubules and actin filaments in living Drosophila embryos. Development 103, 675-686.

Knox, A. L. and Brown, N. H. (2002). Rap1 GTPase Regulation of Adherens Junction Positioning and Cell Adhesion. Science 295, 1285-1288.

Komander, D., Patel, M., Laurin, M., Fradet, N., Pelletier, A., Barford, D. and Côté, J.-F. (2008). An alpha-helical extension of the ELMO1 pleckstrin homology domain mediates direct interaction to DOCK180 and is critical in Rac signaling. Mol. Biol. Cell 19, 4837-4851.

Korotkevich, E., Niwayama, R., Courtois, A., Friese, S., Berger, N., Buchholz, F. and Hiiragi, T. (2017). The Apical Domain Is Required and Sufficient for the First Lineage Segregation in the Mouse Embryo. Dev. Cell 40, 235-247.e7.

Kouranti, I., Sachse, M., Arouche, N., Goud, B. and Echard, A. (2006). Rab35 Regulates an Endocytic Recycling Pathway Essential for the Terminal Steps of Cytokinesis. Curr. Biol. 16, 1719-1725.

Kovar, D. R., Harris, E. S., Mahaffy, R., Higgs, H. N. and Pollard, T. D. (2006). Control of the Assembly of ATP- and ADP-Actin by Formins and Profilin. Cell 124, 423-435.

Krahn, M. P., Klopfenstein, D. R., Fischer, N. and Wodarz, A. (2010a). Membrane Targeting of Bazooka/PAR-3 Is Mediated by Direct Binding to Phosphoinositide Lipids. Curr. Biol. 20, 636-642.

Krahn, M. P., Bückers, J., Kastrup, L. and Wodarz, A. (2010b). Formation of a Bazooka-Stardust complex is essential for plasma membrane polarity in epithelia. J. Cell Biol. 190, 751-760. 
Krämer, H. (2000). The Ups and Downs of Life in an Epithelium. J. Cell Biol. 151, f15-f18.

Kühn, S. and Geyer, M. (2014). Formins as effector proteins of Rho GTPases. Small GTPases 5,.

Kusumi, A., Nakada, C., Ritchie, K., Murase, K., Suzuki, K., Murakoshi, H., Kasai, R. S., Kondo, J. and Fujiwara, T. (2005). Paradigm shift of the plasma membrane concept from the two-dimensional continuum fluid to the partitioned fluid: high-speed single-molecule tracking of membrane molecules. Annu. Rev. Biophys. Biomol. Struct. 34, 351-378.

Lammel, U., Bechtold, M., Risse, B., Berh, D., Fleige, A., Bunse, I., Jiang, X., Klämbt, C. and Bogdan, S. (2014). The Drosophila FHOD1-like formin Knittrig acts through Rok to promote stress fiber formation and directed macrophage migration during the cellular immune response. Development 141, 1366-1380.

Lang, C. F. and Munro, E. (2017). The PAR proteins: from molecular circuits to dynamic self-stabilizing cell polarity. Development 144, 3405-3416.

Laprise, P. and Tepass, U. (2011). Novel insights into epithelial polarity proteins in Drosophila. Trends Cell Biol. 21, 401-408.

Lecuit, T. (2004). Junctions and vesicular trafficking during Drosophila cellularization. J. Cell Sci. 117, 3427-3433.

Lecuit, T. and Wieschaus, E. (2000). Polarized Insertion of New Membrane from a Cytoplasmic Reservoir during Cleavage of the Drosophila Embryo. J. Cell Biol. 150, 849-860.

Lecuit, T., Samanta, R. and Wieschaus, E. (2002). slam encodes a developmental regulator of polarized membrane growth during cleavage of the Drosophila embryo. Dev. Cell 2, 425-436.

Lee, J. H., Cho, K. S., Lee, J., Kim, D., Lee, S.-B., Yoo, J., Cha, G.-H. and Chung, J. (2002). Drosophila PDZ-GEF, a Guanine Nucleotide Exchange Factor for Rap1 GTPase, Reveals a Novel Upstream Regulatory Mechanism in the Mitogen-Activated Protein Kinase Signaling Pathway. Mol. Cell. Biol. 22, 7658-7666.

Lee, O.-K., Frese, K. K., James, J. S., Chadda, D., Chen, Z.-H., Javier, R. T. and Cho, K.-O. (2003). Discs-Large and Strabismus are functionally linked to plasma membrane formation. Nat. Cell Biol. 5, 987-993.

Lehmann, R. and Tautz, D. (1994). Chapter 30 In Situ Hybridization to RNA. In Methods in Cell Biology (ed. Goldstein, L. S. B.) and Fyrberg, E. A.), pp. 575-598. Academic Press.

Li, F. and Higgs, H. N. (2003). The Mouse Formin mDia1 Is a Potent Actin Nucleation Factor Regulated by Autoinhibition. Curr. Biol. 13, 13351340.

Li, Y., Zhu, X., Zeng, Y., Wang, J., Zhang, X., Ding, Y. and Liang, L. (2010). FMNL2 Enhances Invasion of Colorectal Carcinoma by Inducing Epithelial-Mesenchymal Transition. Mol. Cancer Res. 8, 1579-1590. 
Linnemann, T., Geyer, M., Jaitner, B. K., Block, C., Kalbitzer, H. R., Wittinghofer, A. and Herrmann, C. (1999). Thermodynamic and Kinetic Characterization of the Interaction between the Ras Binding Domain of AF6 and Members of the Ras Subfamily. J. Biol. Chem. 274, 13556-13562.

Liu, B. and Grosshans, J. (2017). Link of Zygotic Genome Activation and Cell Cycle Control. In Zygotic Genome Activation, pp. 11-30. Humana Press, New York, NY.

Loppin, B., Docquier, M., Bonneton, F. and Couble, P. (2000). The Maternal Effect Mutation sésame Affects the Formation of the Male Pronucleus in Drosophila melanogaster. Dev. Biol. 222, 392-404.

Lu, Q. and Adler, P. N. (2015). The diaphanous Gene of Drosophila Interacts Antagonistically with multiple wing hairs and Plays a Key Role in Wing Hair Morphogenesis. PLoS ONE 10,.

Luschnig, S., Moussian, B., Krauss, J., Desjeux, I., Perkovic, J. and NüssleinVolhard, C. (2004). An F1 genetic screen for maternal-effect mutations affecting embryonic pattern formation in Drosophila melanogaster. Genetics 167, 325-342.

Lv, Z. and Großhans, J. (2016). A Radial Actin Network in Apical Constriction. Dev. Cell 39, 280-282.

Margaron, Y., Fradet, N. and Côté, J.-F. (2013). ELMO Recruits Actin Crosslinking Family 7 (ACF7) at the Cell Membrane for Microtubule Capture and Stabilization of Cellular Protrusions. J. Biol. Chem. 288, 1184-1199.

Markstein, M., Pitsouli, C., Villalta, C., Celniker, S. E. and Perrimon, N. (2008). Exploiting position effects and the gypsy retrovirus insulator to engineer precisely expressed transgenes. Nat. Genet. 40, 476-483.

Matusek, T., Djiane, A., Jankovics, F., Brunner, D., Mlodzik, M. and Mihály, J. (2006). The Drosophila formin DAAM regulates the tracheal cuticle pattern through organizing the actin cytoskeleton. Dev. Camb. Engl. 133, 957-966.

Mavrakis, M., Rikhy, R. and Lippincott-Schwartz, J. (2009). Plasma membrane polarity and compartmentalization are established before cellularization in the fly embryo. Dev. Cell 16, 93-104.

Mazumdar, A. and Mazumdar, M. (2002). How one becomes many: blastoderm cellularization in Drosophila melanogaster. BioEssays News Rev. Mol. Cell. Dev. Biol. 24, 1012-1022.

McGill, M. A., McKinley, R. F. A. and Harris, T. J. C. (2009). Independent cadherin-catenin and Bazooka clusters interact to assemble adherens junctions. J. Cell Biol. 185, 787-796.

McKinley, R. F. A. and Harris, T. J. C. (2012). Displacement of basolateral Bazooka/PAR-3 by regulated transport and dispersion during epithelial polarization in Drosophila. Mol. Biol. Cell 23, 4465-4471.

Molnár, I., Migh, E., Szikora, S., Kalmár, T., Végh, A. G., Deák, F., Barkó, S., Bugyi, B., Orfanos, Z., Kovács, J., et al. (2014). DAAM is required for 
thin filament formation and Sarcomerogenesis during muscle development in Drosophila. PLoS Genet. 10, e1004166.

Morais-de-Sá, E., Mirouse, V. and St Johnston, D. (2010). aPKC Phosphorylation of Bazooka Defines the Apical/Lateral Border in Drosophila Epithelial Cells. Cell 141, 509-523.

Morin, X., Daneman, R., Zavortink, M. and Chia, W. (2001). A protein trap strategy to detect GFP-tagged proteins expressed from their endogenous loci in Drosophila. Proc. Natl. Acad. Sci. U. S. A. 98, 1505015055.

Morishita, K., Anh Suong, D. N., Yoshida, H. and Yamaguchi, M. (2017). The Drosophila DOCK family protein Sponge is required for development of the air sac primordium. Exp. Cell Res. 354, 95-102.

Moseley, J. B., Sagot, I., Manning, A. L., Xu, Y., Eck, M. J., Pellman, D. and Goode, B. L. (2004). A Conserved Mechanism for Bni1- and mDia1induced Actin Assembly and Dual Regulation of Bni1 by Bud6 and Profilin. Mol. Biol. Cell 15, 896-907.

Mullins, R. D., Heuser, J. A. and Pollard, T. D. (1998). The interaction of Arp2/3 complex with actin: Nucleation, high affinity pointed end capping, and formation of branching networks of filaments. Proc. Natl. Acad. Sci. U. S. A. 95, 6181-6186.

Munjal, A. and Lecuit, T. (2014). Actomyosin networks and tissue morphogenesis. Development 141, 1789-1793.

Munjal, A., Philippe, J.-M., Munro, E. and Lecuit, T. (2015). A self-organized biomechanical network drives shape changes during tissue morphogenesis. Nature 524, 351-355.

Munro, E., Nance, J. and Priess, J. R. (2004). Cortical Flows Powered by Asymmetrical Contraction Transport PAR Proteins to Establish and Maintain Anterior-Posterior Polarity in the Early C. elegans Embryo. Dev. Cell 7, 413-424.

Nance, J. (2014). Getting to know your neighbor: Cell polarization in early embryos. J Cell Biol 206, 823-832.

Nance, J. and Zallen, J. A. (2011). Elaborating polarity: PAR proteins and the cytoskeleton. Dev. Camb. Engl. 138, 799-809.

Ou, J., He, Y., Xiao, X., Yu, T.-M., Chen, C., Gao, Z. and Ho, M. S. (2014). Glial cells in neuronal development: recent advances and insights from $<$ Emphasis Type="Italic" $>$ Drosophila melanogaster $</$ Emphasis $>$. Neurosci. Bull. 30, 584-594.

Padash Barmchi, M., Rogers, S. and Häcker, U. (2005). DRhoGEF2 regulates actin organization and contractility in the Drosophila blastoderm embryo. J. Cell Biol. 168, 575-585.

Pataki, C., Matusek, T., Kurucz, É., Andó, I., Jenny, A. and Mihály, J. (2010). Drosophila Rab23 Is Involved in the Regulation of the Number and Planar Polarization of the Adult Cuticular Hairs. Genetics 184, 10511065. 
Peel, N., Stevens, N. R., Basto, R. and Raff, J. W. (2007). Overexpressing Centriole-Replication Proteins In Vivo Induces Centriole Overduplication and De Novo Formation. Curr. Biol. 17, 834-843.

Petrella, L. N., Smith-Leiker, T. and Cooley, L. (2007). The Ovhts polyprotein is cleaved to produce fusome and ring canal proteins required for Drosophila oogenesis. Development 134, 703-712.

Pinheiro, D., Hannezo, E., Herszterg, S., Bosveld, F., Gaugue, I., Balakireva, M., Wang, Z., Cristo, I., Rigaud, S. U., Markova, O., et al. (2017). Transmission of cytokinesis forces via E-cadherin dilution and actomyosin flows. Nature 545, 103-107.

Pollard, T. D., Blanchoin, L. and Mullins, R. D. (2000). Molecular Mechanisms Controlling Actin Filament Dynamics in Nonmuscle Cells. Annu. Rev. Biophys. Biomol. Struct. 29, 545-576.

Postner, M. A. and Wieschaus, E. F. (1994). The nullo protein is a component of the actin-myosin network that mediates cellularization in Drosophila melanogaster embryos. J Cell Sci 107, 1863-1873.

Postner, M. A., Miller, K. G. and Wieschaus, E. F. (1992). Maternal effect mutations of the sponge locus affect actin cytoskeletal rearrangements in Drosophila melanogaster embryos. J. Cell Biol. 119, 1205-1218.

Pring, M., Evangelista, M., Boone, C., Yang, C. and Zigmond, S. H. (2003). Mechanism of Formin-Induced Nucleation of Actin Filaments. Biochemistry 42, 486-496.

Raff, J. W. and Glover, D. M. (1989). Centrosomes, and not nuclei, initiate pole cell formation in Drosophila embryos. Cell 57, 611-619.

Reversi, A., Loeser, E., Subramanian, D., Schultz, C. and De Renzis, S. (2014). Plasma membrane phosphoinositide balance regulates cell shape during Drosophila embryo morphogenesis. J. Cell Biol. 205, 395-408.

Riggs, B., Rothwell, W., Mische, S., Hickson, G. R. X., Matheson, J., Hays, T. S., Gould, G. W. and Sullivan, W. (2003). Actin cytoskeleton remodeling during early Drosophila furrow formation requires recycling endosomal components Nuclear-fallout and Rab11. J Cell Biol 163, 143-154.

Rikhy, R., Mavrakis, M. and Lippincott-Schwartz, J. (2015). Dynamin regulates metaphase furrow formation and plasma membrane compartmentalization in the syncytial Drosophila embryo. Biol. Open 4, 301-311.

Robertson, A. S., Smythe, E. and Ayscough, K. R. (2009). Functions of actin in endocytosis. Cell. Mol. Life Sci. 66, 2049-2065.

Royou, A., Sullivan, W. and Karess, R. (2002). Cortical recruitment of nonmuscle myosin II in early syncytial Drosophila embryos. J. Cell Biol. $158,127-137$.

Sawyer, J. K., Harris, N. J., Slep, K. C., Gaul, U. and Peifer, M. (2009). The Drosophila afadin homologue Canoe regulates linkage of the actin 
cytoskeleton to adherens junctions during apical constriction. J. Cell Biol. 186, 57-73.

Schmidt, A. and Grosshans, J. (2018). Dynamics of cortical domains in early Drosophila development. J Cell Sci 131, jcs212795.

Schmidt, A., Lv, Z. and Großhans, J. (2018). ELMO and Sponge specify subapical restriction of Canoe and formation of the subapical domain in early Drosophila embryos. Development 145, dev157909.

Schweisguth, F., Lepesant, J. A. and Vincent, A. (1990). The serendipity alpha gene encodes a membrane-associated protein required for the cellularization of the Drosophila embryo. Genes Dev. 4, 922-931.

Shekhar, S., Kerleau, M., Kühn, S., Pernier, J., Romet-Lemonne, G., Jégou, A. and Carlier, M.-F. (2015). Formin and capping protein together embrace the actin filament in a ménage à trois. Nat. Commun. 6,.

Sherlekar, A. and Rikhy, R. (2016). Syndapin promotes pseudocleavage furrow formation by actin organization in the syncytial Drosophila embryo. Mol. Biol. Cell 27, 2064-2079.

Sherlekar, A. and Rikhy, R. (2017). Syndapin bridges the membranecytoskeleton divide during furrow extension. Commun. Integr. Biol. 10,

Shewan, A., Eastburn, D. J. and Mostov, K. (2011). Phosphoinositides in Cell Architecture. Cold Spring Harb. Perspect. Biol. 3,

Shirinian, M., Grabbe, C., Popovic, M., Varshney, G., Hugosson, F., Bos, H., Rehmann, H. and Palmer, R. H. (2010). The Rap1 Guanine Nucleotide Exchange Factor C3G Is Required for Preservation of Larval Muscle Integrity in Drosophila melanogaster. PLoS ONE 5,

Simpson, L. and Wieschaus, E. (1990). Zygotic activity of the nullo locus is required to stabilize the actin-myosin network during cellularization in Drosophila. Development 110, 851-863.

Sokac, A. M. and Wieschaus, E. (2008a). Local Actin-Dependent Endocytosis Is Zygotically Controlled to Initiate Drosophila Cellularization. Dev. Cell 14, 775-786.

Sokac, A. M. and Wieschaus, E. (2008b). Zygotically controlled F-actin establishes cortical compartments to stabilize furrows during Drosophila cellularization. J. Cell Sci. 121, 1815-1824.

Spahn, P., Ott, A. and Reuter, R. (2012). The PDZ-GEF protein Dizzy regulates the establishment of adherens junctions required for ventral furrow formation in Drosophila. J Cell Sci 125, 3801-3812.

Stevenson, V. A., Kramer, J., Kuhn, J. and Theurkauf, W. E. (2001). Centrosomes and the Scrambled protein coordinate microtubuleindependent actin reorganization. Nat. Cell Biol. 3, 68-75.

Stevenson, V., Hudson, A., Cooley, L. and Theurkauf, W. E. (2002). Arp2/3Dependent Psuedocleavage Furrow Assembly in Syncytial Drosophila Embryos. Curr. Biol. 12, 705-711. 
Stork, T., Bernardos, R. and Freeman, M. R. (2012). Analysis of Glial Cell Development and Function in Drosophila. Cold Spring Harb. Protoc. 2012, pdb.top067587.

Su, J., Chow, B., Boulianne, G. L. and Wilde, A. (2013). The BAR domain of amphiphysin is required for cleavage furrow tip-tubule formation during cellularization in Drosophila embryos. Mol. Biol. Cell 24, 14441453.

Sullivan, W. and Theurkauf, W. E. (1995). The cytoskeleton and morphogenesis of the early Drosophila embryo. Curr. Opin. Cell Biol. 7, 18-22.

Sung, H., Spangenberg, S., Vogt, N. and Großhans, J. (2013). Number of Nuclear Divisions in the Drosophila Blastoderm Controlled by Onset of Zygotic Transcription. Curr. Biol. 23, 133-138.

Suzuki, A. and Ohno, S. (2006). The PAR-aPKC system: lessons in polarity. J. Cell Sci. 119, 979-987.

Tanaka, H., Takasu, E., Aigaki, T., Kato, K., Hayashi, S. and Nose, A. (2004). Formin3 is required for assembly of the F-actin structure that mediates tracheal fusion in Drosophila. Dev. Biol. 274, 413-425.

Tanentzapf, G. and Tepass, U. (2003). Interactions between the crumbs, lethal giant larvae and bazooka pathways in epithelial polarization. Nat. Cell Biol. 5, 46-52.

Tepass, U. (2012). The Apical Polarity Protein Network in Drosophila Epithelial Cells: Regulation of Polarity, Junctions, Morphogenesis, Cell Growth, and Survival. Annu. Rev. Cell Dev. Biol. 28, 655-685.

Terasaki, M., Chen, L. B. and Fujiwara, K. (1986). Microtubules and the endoplasmic reticulum are highly interdependent structures. J. Cell Biol. 103, 1557-1568.

Thomas, G. H. and Williams, J. A. (1999). Dynamic rearrangement of the spectrin membrane skeleton during the generation of epithelial polarity in Drosophila. J Cell Sci 112, 2843-2852.

Toret, C. P., Shivakumar, P. C., Lenne, P.-F. and Bivic, A. L. (2018). The ELMO-MBC complex and RhoGAP19D couple Rho family GTPases during mesenchymal-to-epithelial-like transitions. Development 145, dev157495.

Turner, F. R. and Mahowald, A. P. (1976). Scanning electron microscopy of Drosophila embryogenesis. Dev. Biol. 50, 95-108.

Vinot, S., Le, T., Ohno, S., Pawson, T., Maro, B. and Louvet-Vallée, S. (2005). Asymmetric distribution of PAR proteins in the mouse embryo begins at the 8-cell stage during compaction. Dev. Biol. 282, 307-319.

Wallar, B. J. and Alberts, A. S. (2003). The formins: active scaffolds that remodel the cytoskeleton. Trends Cell Biol. 13, 435-446.

Wang, Y.-C., Khan, Z. and Wieschaus, E. F. (2013). Distinct Rap1 activity states control the extent of epithelial invagination via $\alpha$-Catenin. Dev. Cell 25, 299-309. 
Warn, R. M., Bullard, B. and Magrath, R. (1980). Changes in the distribution of cortical myosin during the cellularization of the Drosophila embryo. Development 57, 167-176.

Warn, R. M., Magrath, R. and Webb, S. (1984). Distribution of F-actin during cleavage of the Drosophila syncytial blastoderm. J. Cell Biol. 98, 156-162.

Warn, R. M., Flegg, L. and Warn, A. (1987). An investigation of microtubule organization and functions in living Drosophila embryos by injection of a fluorescently labeled antibody against tyrosinated alpha-tubulin. J. Cell Biol. 105, 1721-1730.

Waterman-Storer, C. M. and Salmon, E. D. (1998). Endoplasmic reticulum membrane tubules are distributed by microtubules in living cells using three distinct mechanisms. Curr. Biol. 8, 798-807.

Webb, R. L., Zhou, M.-N. and McCartney, B. M. (2009). A novel role for an APC2-Diaphanous complex in regulating actin organization in Drosophila. Development 136, 1283-1293.

Wenzl, C., Yan, S., Laupsien, P. and Grosshans, J. (2010). Localization of RhoGEF2 during Drosophila cellularization is developmentally controlled by Slam. Mech. Dev. 127, 371-384.

Winkler, F., Gummalla, M., Künneke, L., Lv, Z., Zippelius, A., Aspelmeier, T. and Grosshans, J. (2015). Fluctuation Analysis of Centrosomes Reveals a Cortical Function of Kinesin-1. Biophys. J. 109, 856-868.

Xue, F., Janzen, D. M. and Knecht, D. A. (2010). Contribution of Filopodia to Cell Migration: A Mechanical Link between Protrusion and Contraction. Int. J. Cell Biol. 2010,.

Yajnik, V., Paulding, C., Sordella, R., McClatchey, A. I., Saito, M., Wahrer, D. C. R., Reynolds, P., Bell, D. W., Lake, R., van den Heuvel, S., et al. (2003). DOCK4, a GTPase activator, is disrupted during tumorigenesis. Cell 112, 673-684.

Yamanaka, T., Horikoshi, Y., Izumi, N., Suzuki, A., Mizuno, K. and Ohno, S. (2006). Lgl mediates apical domain disassembly by suppressing the PAR-3-aPKC-PAR-6 complex to orient apical membrane polarity. J. Cell Sci. 119, 2107-2118.

Yan, S., Lv, Z., Winterhoff, M., Wenzl, C., Zobel, T., Faix, J., Bogdan, S. and Grosshans, J. (2013). The F-BAR protein Cip4/Toca-1 antagonizes the formin Diaphanous in membrane stabilization and compartmentalization. J. Cell Sci. 126, 1796-1805.

Yan, S., Acharya, S., Gröning, S. and Großhans, J. (2017). Slam protein dictates subcellular localization and translation of its own mRNA. PLOS Biol. 15, e2003315.

Yasuda, G. K., Baker, J. and Schubiger, G. (1991). Independent roles of centrosomes and DNA in organizing the Drosophila cytoskeleton. Development 111, 379-391. 
Young, P. E., Pesacreta, T. C. and Kiehart, D. P. (1991). Dynamic changes in the distribution of cytoplasmic myosin during Drosophila embryogenesis. Development 111, 1-14.

Yuan, K., Seller, C. A., Shermoen, A. W. and O'Farrell, P. H. (2016). Timing the Drosophila Mid-Blastula Transition: A Cell Cycle-Centered View. Trends Genet. TIG 32,.

Zacharias, D. A., Violin, J. D., Newton, A. C. and Tsien, R. Y. (2002). Partitioning of lipid-modified monomeric GFPs into membrane microdomains of live cells. Science 296, 913-916.

Zallen, J. A., Cohen, Y., Hudson, A. M., Cooley, L., Wieschaus, E. and Schejter, E. D. (2002). SCAR is a primary regulator of Arp2/3dependent morphological events in Drosophila. J. Cell Biol. 156, 689701.

Zeitler, J., Hsu, C. P., Dionne, H. and Bilder, D. (2004). Domains controlling cell polarity and proliferation in the Drosophila tumor suppressor Scribble. J Cell Biol 167, 1137-1146.

Zerial, M. and McBride, H. (2001). Rab proteins as membrane organizers. Nat. Rev. Mol. Cell Biol. 2, 107-117.

Zhang, J., Fonovic, M., Suyama, K., Bogyo, M. and Scott, M. P. (2009). Rab35 Controls Actin Bundling by Recruiting Fascin as an Effector Protein. Science 325, 1250-1254. 


\section{Curriculum vitae}

\section{Personal data}

$\begin{array}{ll}\text { Name } & \text { Anja Schmidt } \\ \text { Date of birth } & 24.10 .1984 \\ \text { Place of birth } & \text { Bad Hersfeld } \\ \text { Address } & \text { Felix-Klein-Str. 9, 37083 Göttingen } \\ \text { Email } & \text { a.schmidt84@arcor.de }\end{array}$

\section{Education and Career}

$\begin{array}{ll}\text { From 05/2013 } & \text { PhD student at the Department of Developmental } \\ & \text { Biology, Georg-August University Göttingen } \\ & \text { PhD thesis title: “Regulation and function of actin } \\ & \text { nucleators Dia and FMNL in the early Drosophila } \\ & \text { embryo.” } \\ & \text { Advisor: Prof. Dr. Jörg Großhans; Georg-August } \\ & \text { University School of Science (GAUSS) program } \\ & \text { Biology } \\ & \text { Marketing Manager at MoBiTec, GmbH, Göttingen } \\ 11 / 2009-05 / 2013 & \text { Study of Biology, Georg-August University } \\ 10 / 2004-11 / 2009 & \text { Göttingen } \\ & \text { Title of Diploma thesis: “Untersuchung zur zellulären } \\ & \text { Kompetition von Keimbahnstammzellen in } \\ & \text { Drosophila melanogaster." } \\ & \text { Advisor: Prof. Dr. Herbert Jäckle, Molecular } \\ & \text { Developmental Biology, Max-Planck-Institute for } \\ \text { biophysical Chemistry, Göttingen } \\ \text { Abitur at Gymnasiale Oberstufe, Modellschule } \\ \text { Obersberg, Bad Hersfeld } \\ \text { Gesamtschule Obersberg, Bad Hersfeld } \\ 08 / 2001-07 / 2004=07 / 2001\end{array}$




\section{Appendix}

Table 6 Normalized values of fluorescence intensities measured for Figure 8B.

\begin{tabular}{|c|c|c|c|c|c|c|c|c|}
\hline & Furrov & & Furrow & & Furrow & & Averag & \\
\hline $\begin{array}{l}a-b \\
\text { pos. }\end{array}$ & $\begin{array}{l}\text { Cno } \\
3 \mathrm{mi} \\
\mathrm{n}\end{array}$ & $\begin{array}{l}\text { Slam } \\
3 \text { min }\end{array}$ & $\begin{array}{l}\text { Cno } \\
3 \mathrm{~min}\end{array}$ & $\begin{array}{l}\text { Slam } \\
3 \mathrm{~min}\end{array}$ & $\begin{array}{l}\text { Cno } \\
3 \mathrm{~min}\end{array}$ & $\begin{array}{l}\text { Slam } \\
3 \mathrm{~min}\end{array}$ & $\begin{array}{l}\text { Cno } \\
3 \mathrm{~min}\end{array}$ & $\begin{array}{l}\text { Slam } \\
3 \mathrm{~min}\end{array}$ \\
\hline$[\mu \mathrm{m}]$ & $\begin{array}{l}\text { Nor } \\
\mathrm{m} \\
\text { [a.u.] }\end{array}$ & $\begin{array}{l}\text { Norm } \\
\text { [a.u.] }\end{array}$ & $\begin{array}{l}\text { Norm } \\
\text { [a.u.] }\end{array}$ & $\begin{array}{l}\text { Norm } \\
\text { [a.u.] }\end{array}$ & $\begin{array}{l}\text { Norm } \\
\text { [a.u.] }\end{array}$ & $\begin{array}{l}\text { Norm } \\
\text { [a.u.] }\end{array}$ & $\begin{array}{l}\text { Norm } \\
\text { [a.u.] }\end{array}$ & $\begin{array}{l}\text { Norm } \\
\text { [a.u.] }\end{array}$ \\
\hline 0.00 & 0.62 & 0.31 & 0.38 & 0.25 & 0.57 & 0.29 & 0.52 & 0.28 \\
\hline 0.50 & 0.57 & 0.24 & 0.62 & 0.20 & 0.59 & 0.26 & 0.60 & 0.23 \\
\hline 1.00 & 0.59 & 0.27 & 0.64 & 0.27 & 0.73 & 0.23 & 0.66 & 0.26 \\
\hline 1.50 & 0.85 & 0.38 & 1.00 & 0.60 & 0.93 & 0.33 & 0.94 & 0.44 \\
\hline 2.00 & 0.86 & 0.40 & 0.94 & 0.57 & 0.86 & 0.40 & 0.89 & 0.46 \\
\hline 2.50 & 0.69 & 0.38 & 0.83 & 0.48 & 0.65 & 0.39 & 0.73 & 0.42 \\
\hline 3.00 & 0.56 & 0.31 & 0.64 & 0.38 & 0.44 & 0.30 & 0.54 & 0.33 \\
\hline 3.50 & 0.28 & 0.20 & 0.26 & 0.23 & 0.23 & 0.19 & 0.26 & 0.21 \\
\hline 4.00 & 0.12 & 0.14 & 0.15 & 0.21 & 0.14 & 0.16 & 0.14 & 0.17 \\
\hline 4.50 & 0.15 & 0.14 & 0.14 & 0.24 & 0.10 & 0.12 & 0.13 & 0.17 \\
\hline 5.00 & 0.12 & 0.11 & 0.14 & 0.25 & 0.12 & 0.13 & 0.13 & 0.16 \\
\hline 5.50 & 0.12 & 0.14 & 0.11 & 0.23 & 0.08 & 0.10 & 0.10 & 0.15 \\
\hline 6.00 & 0.08 & 0.17 & 0.07 & 0.25 & 0.10 & 0.09 & 0.08 & 0.17 \\
\hline 6.50 & 0.09 & 0.14 & 0.09 & 0.17 & 0.07 & 0.18 & 0.08 & 0.16 \\
\hline 7.00 & 0.09 & 0.15 & 0.10 & 0.15 & 0.10 & 0.15 & 0.10 & 0.15 \\
\hline 7.50 & 0.10 & 0.14 & 0.07 & 0.19 & 0.08 & 0.14 & 0.08 & 0.16 \\
\hline 8.00 & 0.08 & 0.15 & 0.10 & 0.24 & 0.08 & 0.14 & 0.09 & 0.18 \\
\hline 8.50 & 0.09 & 0.18 & 0.10 & 0.20 & 0.09 & 0.16 & 0.09 & 0.18 \\
\hline 9.00 & 0.11 & 0.21 & 0.10 & 0.23 & 0.12 & 0.16 & 0.11 & 0.20 \\
\hline
\end{tabular}


Normalized values of fluorescence intensities measured for Figure 8B

- continuation

\begin{tabular}{|c|c|c|c|c|c|c|c|c|}
\hline $\begin{array}{l}\text { a-b } \\
\text { pos. }\end{array}$ & $\begin{array}{l}\text { Cno } \\
3 \mathrm{mi} \\
\mathrm{n}\end{array}$ & $\begin{array}{l}\text { Slam } \\
3 \text { min }\end{array}$ & $\begin{array}{l}\text { Cno } \\
3 \mathrm{~min}\end{array}$ & $\begin{array}{l}\text { Slam } \\
3 \mathrm{~min}\end{array}$ & $\begin{array}{l}\text { Cno } \\
3 \mathrm{~min}\end{array}$ & $\begin{array}{l}\text { Slam } \\
3 \mathrm{~min}\end{array}$ & $\begin{array}{l}\text { Cno } \\
8 \mathrm{~min}\end{array}$ & $\begin{array}{l}\text { Slam } \\
8 \mathrm{~min}\end{array}$ \\
\hline$[\mu \mathrm{m}]$ & $\begin{array}{l}\text { Nor } \\
\mathrm{m} \\
\text { [a.u.] }\end{array}$ & $\begin{array}{l}\text { Norm } \\
\text { [a.u.] }\end{array}$ & $\begin{array}{l}\text { Norm } \\
\text { [a.u.] }\end{array}$ & $\begin{array}{l}\text { Norm } \\
\text { [a.u.] }\end{array}$ & $\begin{array}{l}\text { Norm } \\
\text { [a.u.] }\end{array}$ & $\begin{array}{l}\text { Norm } \\
\text { [a.u.] }\end{array}$ & $\begin{array}{l}\text { Norm } \\
\text { [a.u.] }\end{array}$ & $\begin{array}{l}\text { Norm } \\
\text { [a.u.] }\end{array}$ \\
\hline 0.00 & 0.58 & 0.22 & 0.46 & 0.16 & 0.63 & 0.24 & 0.56 & 0.21 \\
\hline 0.50 & 0.61 & 0.26 & 0.55 & 0.19 & 0.64 & 0.20 & 0.60 & 0.22 \\
\hline 1.00 & 0.65 & 0.29 & 0.68 & 0.19 & 0.67 & 0.27 & 0.67 & 0.25 \\
\hline 1.50 & 0.94 & 0.42 & 0.79 & 0.36 & 0.75 & 0.34 & 0.83 & 0.37 \\
\hline 2.00 & 0.95 & 0.51 & 1.00 & 0.39 & 0.85 & 0.44 & 0.94 & 0.45 \\
\hline 2.50 & 0.88 & 0.64 & 0.94 & 0.48 & 0.84 & 0.44 & 0.88 & 0.52 \\
\hline 3.00 & 0.73 & 0.93 & 0.78 & 0.59 & 0.68 & 0.57 & 0.73 & 0.70 \\
\hline 3.50 & 0.53 & 1.00 & 0.42 & 0.73 & 0.42 & 0.87 & 0.46 & 0.87 \\
\hline 4.00 & 0.22 & 0.53 & 0.23 & 0.40 & 0.21 & 0.73 & 0.22 & 0.56 \\
\hline 4.50 & 0.16 & 0.38 & 0.16 & 0.30 & 0.18 & 0.40 & 0.17 & 0.36 \\
\hline 5.00 & 0.17 & 0.27 & 0.14 & 0.25 & 0.16 & 0.32 & 0.16 & 0.28 \\
\hline 5.50 & 0.13 & 0.23 & 0.12 & 0.19 & 0.12 & 0.32 & 0.12 & 0.25 \\
\hline 6.00 & 0.10 & 0.19 & 0.11 & 0.17 & 0.10 & 0.19 & 0.10 & 0.18 \\
\hline 6.50 & 0.08 & 0.18 & 0.09 & 0.14 & 0.11 & 0.18 & 0.09 & 0.17 \\
\hline 7.00 & 0.11 & 0.21 & 0.11 & 0.17 & 0.08 & 0.18 & 0.10 & 0.19 \\
\hline 7.50 & 0.14 & 0.21 & 0.09 & 0.18 & 0.10 & 0.16 & 0.11 & 0.19 \\
\hline 8.00 & 0.10 & 0.16 & 0.08 & 0.17 & 0.10 & 0.18 & 0.09 & 0.17 \\
\hline 8.50 & 0.09 & 0.28 & 0.08 & 0.18 & 0.10 & 0.21 & 0.09 & 0.22 \\
\hline 9.00 & 0.10 & 0.25 & 0.10 & 0.19 & 0.09 & 0.24 & 0.10 & 0.23 \\
\hline
\end{tabular}


Table 7 Normalized fluorescence intensity measured for Figure 8C

\begin{tabular}{|c|c|c|c|}
\hline 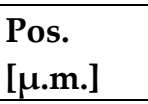 & $\begin{array}{l}\text { Norm. fl } \\
\text { of Cno si }\end{array}$ & $\begin{array}{l}\text { orescence } \\
\text { gnal [a.u.] }\end{array}$ & intensity \\
\hline & $3 \mathrm{~min}$ & $8 \mathrm{~min}$ & $13 \mathrm{~min}$ \\
\hline-2.69 & 0.19 & 0.10 & 0.16 \\
\hline-2.58 & 0.27 & 0.06 & 0.10 \\
\hline-2.47 & 0.11 & 0.07 & 0.05 \\
\hline-2.36 & 0.09 & 0.04 & 0.03 \\
\hline-2.24 & 0.07 & 0.12 & 0.09 \\
\hline-2.13 & 0.08 & 0.07 & 0.15 \\
\hline-2.02 & 0.16 & 0.18 & 0.06 \\
\hline-1.91 & 0.06 & 0.11 & 0.07 \\
\hline-1.79 & 0.06 & 0.15 & 0.03 \\
\hline-1.68 & 0.15 & 0.10 & 0.10 \\
\hline-1.57 & 0.17 & 0.14 & 0.14 \\
\hline-1.46 & 0.16 & 0.23 & 0.23 \\
\hline-1.35 & 0.44 & 0.16 & 0.14 \\
\hline-1.23 & 0.74 & 0.49 & 0.21 \\
\hline-1.12 & 0.78 & 0.42 & 0.22 \\
\hline-1.01 & 0.63 & 0.52 & 0.17 \\
\hline-0.90 & 0.42 & 0.74 & 0.17 \\
\hline-0.79 & 0.55 & 0.56 & 0.27 \\
\hline-0.67 & 0.45 & 0.50 & 0.33 \\
\hline-0.56 & 0.57 & 0.73 & 0.21 \\
\hline-0.45 & 0.35 & 0.86 & 0.23 \\
\hline-0.34 & 0.57 & 0.89 & 0.08 \\
\hline-0.22 & 0.57 & 1.00 & 0.39 \\
\hline-0.11 & 0.37 & 0.77 & 0.57 \\
\hline 0.00 & 0.53 & 0.65 & 1.00 \\
\hline 0.11 & 0.64 & 0.49 & 0.80 \\
\hline 0.22 & 0.41 & 0.37 & 0.48 \\
\hline 0.34 & 0.35 & 0.45 & 0.47 \\
\hline 0.45 & 0.40 & 0.10 & 0.40 \\
\hline 0.56 & 0.50 & 0.15 & 0.11 \\
\hline 0.67 & 0.72 & 0.08 & 0.20 \\
\hline 0.79 & 0.55 & 0.09 & 0.10 \\
\hline 0.90 & 0.52 & 0.16 & 0.10 \\
\hline 1.01 & 1.00 & 0.09 & 0.05 \\
\hline 1.12 & 0.76 & 0.07 & 0.06 \\
\hline 1.23 & 0.51 & 0.14 & 0.21 \\
\hline
\end{tabular}

\begin{tabular}{|c|c|c|c|}
\hline \multirow[t]{2}{*}{$\begin{array}{l}\text { Pos. } \\
\text { [ } \mu . \mathrm{m} .]\end{array}$} & \multicolumn{3}{|c|}{$\begin{array}{l}\text { Norm. fluorescence intensity } \\
\text { of Cno signal [a.u.] }\end{array}$} \\
\hline & $3 \mathrm{~min}$ & $8 \mathrm{~min}$ & $13 \mathrm{~min}$ \\
\hline 1.35 & 0.38 & 0.23 & 0.10 \\
\hline 1.46 & 0.19 & 0.09 & 0.17 \\
\hline 1.57 & 0.08 & 0.09 & 0.09 \\
\hline 1.68 & 0.10 & 0.30 & 0.05 \\
\hline 1.79 & 0.10 & 0.13 & 0.03 \\
\hline 1.91 & 0.07 & 0.03 & 0.07 \\
\hline 2.02 & 0.12 & 0.06 & 0.03 \\
\hline 2.13 & 0.06 & 0.03 & 0.06 \\
\hline 2.24 & 0.12 & 0.05 & 0.11 \\
\hline 2.36 & 0.23 & 0.07 & 0.02 \\
\hline
\end{tabular}


Table 8 Furrow width marked by Canoe measured for Figure 8D

\begin{tabular}{|c|c|c|c|c|}
\hline \multirow{2}{*}{$\begin{array}{l}\text { Time } \\
\text { [min] }\end{array}$} & \multicolumn{3}{|c|}{ Furrow width $[\mu \mathrm{m}]$} & \multirow{2}{*}{$\begin{array}{l}\begin{array}{l}\text { Furrow } \\
\text { width } \\
{[\mu \mathrm{m}]}\end{array} \\
\text { Average }\end{array}$} \\
\hline & Furrow 1 & Furrow 2 & Furrow 3 & \\
\hline 4.00 & 2.42 & 2.65 & 2.27 & 2.44 \\
\hline 5.00 & 2.22 & 1.88 & 1.59 & 1.90 \\
\hline 6.00 & 1.60 & 1.93 & 1.51 & 1.68 \\
\hline 7.00 & 1.18 & 1.26 & 1.27 & 1.24 \\
\hline 8.00 & 1.30 & 0.97 & 0.83 & 1.03 \\
\hline 9.00 & 1.01 & 0.73 & 0.69 & 0.81 \\
\hline 10.00 & 0.67 & 0.58 & 0.60 & 0.62 \\
\hline 11.00 & 0.63 & 0.58 & 0.61 & 0.60 \\
\hline 12.00 & 0.79 & 0.63 & 0.28 & 0.57 \\
\hline 13.00 & 0.56 & 0.53 & 0.52 & 0.54 \\
\hline 14.00 & 0.39 & 0.32 & 0.40 & 0.37 \\
\hline
\end{tabular}

Table 9 Normalized fluorescence intensity of Scrib signal measured for Figure 10C

\begin{tabular}{|c|c|c|c|}
\hline \multirow{2}{*}{$\begin{array}{l}\text { a-b } \\
\text { position } \\
{[\mu \mathrm{m}]}\end{array}$} & \multicolumn{3}{|c|}{$\begin{array}{l}\text { Normalized fluorescence intensity Scrib [a.u.] } 3 \\
\text { min }\end{array}$} \\
\hline & Furrow 1 & Furrow 2 & Furrow 3 \\
\hline 0,00 & 0,53 & 0,63 & 0,67 \\
\hline 1,00 & 0,44 & 0,60 & 0,60 \\
\hline 2,00 & 0,75 & 0,69 & 0,60 \\
\hline 3,00 & 0,26 & 0,23 & 0,21 \\
\hline 4,00 & 0,26 & 0,17 & 0,17 \\
\hline 5,00 & 0,25 & 0,13 & 0,14 \\
\hline 6,00 & 0,17 & 0,11 & 0,09 \\
\hline 7,00 & 0,12 & 0,12 & 0,09 \\
\hline 8,00 & 0,13 & 0,14 & 0,14 \\
\hline 9,00 & 0,13 & 0,14 & 0,17 \\
\hline 10,00 & 0,13 & 0,16 & 0,13 \\
\hline \multirow{2}{*}{$\begin{array}{l}a-b \\
\text { position } \\
{[\mu \mathrm{m}]}\end{array}$} & \multicolumn{3}{|c|}{$\begin{array}{l}\text { Normalized fluorescence intensity Scrib [a.u.] } 8 \\
\text { min }\end{array}$} \\
\hline & Furrow 1 & Furrow 2 & Furrow 3 \\
\hline 0,00 & 0,64 & 0,64 & 0,59 \\
\hline 1,00 & 0,60 & 0,74 & 0,76 \\
\hline 2,00 & 0,64 & 0,71 & 0,75 \\
\hline 3,00 & 0,83 & 0,83 & 1,00 \\
\hline 4,00 & 0,73 & 0,81 & 0,98 \\
\hline 5,00 & 0,49 & 0,59 & 0,82 \\
\hline 6,00 & 0,19 & 0,24 & 0,43 \\
\hline 7,00 & 0,16 & 0,16 & 0,34 \\
\hline 8,00 & 0,14 & 0,11 & 0,14 \\
\hline 9,00 & 0,11 & 0,14 & 0,13 \\
\hline 10,00 & 0,14 & 0,13 & 0,14 \\
\hline
\end{tabular}


Table 10 Normalized fluorescence intensity of Canoe signal measured for Figure 16D

\begin{tabular}{|c|c|c|c|c|c|c|c|c|c|c|}
\hline \multirow{2}{*}{$\begin{array}{l}\text { a-b } \\
\text { position } \\
{[\mu \mathrm{m}]}\end{array}$} & \multicolumn{9}{|c|}{$\begin{array}{l}\text { Wild type } \\
\text { Normalized fluorescence intensity of Canoe signal [a.u.] }\end{array}$} & \multirow[t]{2}{*}{ Average } \\
\hline & E 1 & E 1 & E 1 & E 2 & E 2 & E 2 & E 3 & E 3 & E 3 & \\
\hline-1.51 & 0.37 & 0.17 & & 0.35 & & & & & & 0.30 \\
\hline-1.32 & 0.44 & 0.24 & & 0.37 & 0.26 & 0.37 & & & 0.08 & 0.29 \\
\hline-1.13 & 0.55 & 0.32 & & 0.56 & 0.34 & 0.49 & 0.19 & & 0.12 & 0.37 \\
\hline-0.94 & 0.78 & 0.44 & 0.22 & 0.67 & 0.37 & 0.57 & 0.27 & 0.20 & 0.22 & 0.42 \\
\hline-0.75 & 0.73 & 0.63 & 0.35 & 0.67 & 0.37 & 0.61 & 0.31 & 0.26 & 0.34 & 0.47 \\
\hline-0.56 & 0.80 & 0.74 & 0.52 & 0.59 & 0.47 & 0.62 & 0.34 & 0.36 & 0.52 & 0.55 \\
\hline-0.38 & 0.73 & 0.68 & 0.57 & 0.50 & 0.72 & 0.72 & 0.45 & 0.48 & 0.72 & 0.62 \\
\hline-0.19 & 0.84 & 0.76 & 0.83 & 0.78 & 0.66 & 0.75 & 0.60 & 0.66 & 0.82 & 0.74 \\
\hline 0.00 & 1.00 & 1.00 & 1.00 & 1.00 & 1.00 & 1.00 & 0.95 & 0.99 & 1.00 & 0.99 \\
\hline 0.19 & 0.74 & 0.90 & 0.90 & 0.83 & 0.57 & 0.96 & 1.00 & 1.00 & 0.84 & 0.86 \\
\hline 0.38 & 0.51 & 0.90 & 0.74 & 0.66 & 0.43 & 0.64 & 0.99 & 0.85 & 0.47 & 0.69 \\
\hline 0.56 & 0.39 & 0.92 & 0.67 & 0.47 & 0.42 & 0.52 & 0.85 & 0.54 & 0.36 & 0.57 \\
\hline 0.75 & 0.41 & 0.66 & 0.61 & 0.38 & 0.62 & 0.44 & 0.63 & 0.56 & 0.36 & 0.52 \\
\hline 0.94 & 0.44 & 0.43 & 0.47 & 0.26 & 0.51 & 0.27 & 0.56 & 0.43 & 0.41 & 0.42 \\
\hline 1.13 & 0.36 & 0.38 & 0.41 & 0.29 & 0.46 & 0.36 & 0.39 & 0.31 & 0.30 & 0.36 \\
\hline 1.32 & 0.31 & 0.28 & 0.35 & 0.30 & 0.50 & 0.30 & 0.30 & 0.28 & 0.28 & 0.32 \\
\hline 1.51 & 0.29 & 0.32 & 0.34 & 0.28 & 0.34 & 0.26 & 0.22 & 0.29 & 0.29 & 0.29 \\
\hline 1.69 & 0.26 & 0.31 & 0.33 & 0.27 & 0.24 & 0.31 & 0.25 & 0.32 & 0.31 & 0.29 \\
\hline 1.88 & 0.25 & 0.22 & 0.26 & 0.25 & 0.23 & 0.31 & 0.25 & 0.55 & 0.30 & 0.29 \\
\hline 2.07 & 0.26 & 0.21 & 0.22 & 0.20 & 0.27 & 0.33 & 0.18 & 0.44 & 0.23 & 0.26 \\
\hline 2.26 & 0.29 & 0.28 & 0.27 & 0.18 & 0.30 & 0.38 & 0.18 & 0.24 & 0.18 & 0.26 \\
\hline 2.45 & 0.33 & 0.25 & 0.23 & 0.21 & 0.28 & 0.40 & 0.22 & 0.17 & 0.21 & 0.25 \\
\hline 2.64 & 0.30 & 0.23 & 0.18 & 0.20 & 0.30 & 0.40 & 0.27 & 0.18 & 0.22 & 0.25 \\
\hline 2.82 & 0.23 & 0.18 & 0.26 & 0.27 & 0.33 & 0.47 & 0.25 & 0.20 & 0.26 & 0.27 \\
\hline 3.01 & 0.27 & 0.19 & 0.27 & 0.27 & 0.36 & 0.35 & 0.25 & 0.20 & 0.22 & 0.26 \\
\hline 3.20 & 0.26 & 0.30 & 0.28 & 0.20 & 0.33 & 0.27 & 0.30 & 0.22 & 0.26 & 0.27 \\
\hline 3.39 & & 0.32 & 0.31 & 0.21 & 0.29 & 0.22 & 0.32 & 0.24 & 0.22 & 0.27 \\
\hline 3.58 & & 0.49 & 0.31 & 0.23 & 0.26 & 0.19 & 0.26 & 0.26 & 0.23 & 0.28 \\
\hline 3.77 & & 0.31 & 0.40 & 0.22 & 0.25 & 0.19 & 0.23 & 0.25 & 0.18 & 0.25 \\
\hline 3.95 & & & 0.33 & 0.23 & 0.18 & 0.12 & 0.20 & 0.23 & 0.15 & 0.21 \\
\hline 4.14 & & & 0.29 & 0.24 & 0.17 & & & 0.22 & & 0.23 \\
\hline 4.33 & & & 0.27 & & 0.21 & & & 0.19 & & 0.23 \\
\hline 4.52 & & & & & & & & 0.18 & & 0.18 \\
\hline 4.71 & & & & & & & & 0.16 & & 0.16 \\
\hline
\end{tabular}


Normalized fluorescence intensity of Canoe signal measured for Figure 16D - continuation

\begin{tabular}{|c|c|c|c|c|c|c|c|c|c|c|}
\hline \multirow{2}{*}{$\begin{array}{l}a-b \\
\text { position } \\
{[\mu \mathrm{m}]}\end{array}$} & \multicolumn{9}{|c|}{$\begin{array}{l}\text { ELMO } \\
\text { Normalized fluorescence intensity of Canoe signal [a.u.] }\end{array}$} & \multirow[t]{2}{*}{ Average } \\
\hline & E 1 & E 1 & E 1 & E 2 & E 2 & E 2 & E 3 & E 3 & E 3 & \\
\hline-1.51 & & & 0.29 & & & & & 0.15 & & 0.22 \\
\hline-1.32 & & & 0.35 & & & & 0.12 & 0.25 & & 0.24 \\
\hline-1.13 & & 0.24 & 0.43 & & 0.36 & & 0.18 & 0.47 & & 0.34 \\
\hline-0.94 & 0.25 & 0.36 & 0.50 & & 0.45 & 0.20 & 0.29 & 0.65 & & 0.39 \\
\hline-0.75 & 0.31 & 0.56 & 0.59 & & 0.58 & 0.29 & 0.41 & 0.70 & 0.34 & 0.47 \\
\hline-0.56 & 0.39 & 0.61 & 0.69 & & 0.64 & 0.34 & 0.47 & 0.63 & 0.51 & 0.54 \\
\hline-0.38 & 0.55 & 0.81 & 0.81 & & 0.61 & 0.52 & 0.63 & 0.69 & 0.66 & 0.66 \\
\hline-0.19 & 0.81 & 0.83 & 0.88 & 0.81 & 0.75 & 0.74 & 0.88 & 0.75 & 0.82 & 0.81 \\
\hline 0.00 & 0.91 & 0.90 & 0.97 & 0.96 & 0.97 & 0.91 & 1.00 & 0.94 & 0.97 & 0.95 \\
\hline 0.19 & 0.97 & 0.98 & 0.85 & 0.90 & 1.00 & 0.91 & 0.88 & 0.90 & 1.00 & 0.93 \\
\hline 0.38 & 0.90 & 1.00 & 0.86 & 0.78 & 0.86 & 0.86 & 0.81 & 0.82 & 0.89 & 0.86 \\
\hline 0.56 & 0.91 & 0.94 & 0.82 & 0.67 & 0.74 & 0.86 & 0.68 & 0.75 & 0.89 & 0.81 \\
\hline 0.75 & 0.91 & 0.81 & 0.76 & 0.62 & 0.73 & 0.86 & 0.53 & 0.95 & 0.88 & 0.78 \\
\hline 0.94 & 0.98 & 0.86 & 0.79 & 0.68 & 0.76 & 0.98 & 0.49 & 1.00 & 0.90 & 0.83 \\
\hline 1.13 & 0.87 & 0.81 & 0.78 & 0.76 & \begin{tabular}{|l|}
0.72 \\
\end{tabular} & 1.00 & 0.50 & 0.81 & 0.88 & 0.79 \\
\hline 1.32 & 0.75 & 0.74 & 0.79 & 0.78 & 0.73 & 0.84 & 0.51 & 0.77 & \begin{tabular}{|l|}
0.81 \\
\end{tabular} & 0.75 \\
\hline 1.51 & 0.78 & 0.65 & 0.83 & 0.70 & \begin{tabular}{|l|}
0.72 \\
\end{tabular} & 0.80 & 0.43 & 0.62 & \begin{tabular}{|l}
0.82 \\
\end{tabular} & 0.71 \\
\hline 1.69 & 1.00 & 0.64 & 0.83 & 0.78 & \begin{tabular}{|l|}
0.73 \\
\end{tabular} & 0.73 & 0.50 & 0.67 & 0.76 & 0.74 \\
\hline 1.88 & 0.91 & 0.77 & 0.78 & 0.79 & 0.84 & 0.74 & 0.47 & 0.77 & $\begin{array}{ll}0.82 \\
\end{array}$ & 0.76 \\
\hline 2.07 & 0.87 & 0.80 & 0.78 & 0.70 & 0.92 & 0.83 & 0.48 & 0.79 & 0.87 & 0.78 \\
\hline 2.26 & 0.94 & 0.79 & 0.83 & 0.84 & \begin{tabular}{|l|l}
0.87 \\
\end{tabular} & 0.75 & 0.51 & 0.76 & \begin{tabular}{|l|}
0.82 \\
\end{tabular} & 0.79 \\
\hline 2.45 & 0.98 & 0.86 & 0.94 & 1.00 & \begin{tabular}{|l|}
0.87 \\
\end{tabular} & 0.64 & 0.48 & 0.69 & \begin{tabular}{|l|}
0.79 \\
\end{tabular} & 0.80 \\
\hline 2.64 & 0.87 & 0.83 & 1.00 & 0.89 & 0.86 & 0.59 & 0.53 & 0.57 & \begin{tabular}{|l|}
0.81 \\
\end{tabular} & 0.77 \\
\hline 2.82 & 0.81 & 0.76 & 0.88 & 0.83 & 0.74 & 0.52 & 0.67 & & 0.82 & 0.75 \\
\hline 3.01 & 0.81 & 0.68 & 0.87 & 0.65 & 0.71 & 0.60 & 0.66 & & 0.80 & 0.72 \\
\hline 3.20 & 0.83 & 0.67 & 0.84 & 0.55 & 0.67 & 0.59 & 0.64 & & 0.77 & 0.69 \\
\hline 3.39 & 0.84 & 0.63 & 0.89 & 0.54 & 0.63 & 0.57 & 0.74 & & 0.76 & 0.70 \\
\hline 3.58 & 0.71 & 0.63 & & 0.53 & 0.55 & 0.64 & 0.76 & & 0.80 & 0.66 \\
\hline 3.77 & 0.67 & 0.69 & & 0.51 & 0.64 & 0.63 & 0.70 & & 0.84 & 0.67 \\
\hline 3.95 & 0.68 & 0.71 & & 0.60 & \begin{tabular}{|l}
0.73 \\
\end{tabular} & 0.61 & 0.72 & & 0.86 & 0.70 \\
\hline 4.14 & 0.72 & 0.66 & & 0.67 & 0.84 & 0.74 & 0.75 & & 0.82 & 0.74 \\
\hline 4.33 & 0.68 & 0.67 & & 0.66 & 0.82 & 0.83 & & & & 0.73 \\
\hline 4.52 & 0.64 & & & 0.62 & 0.83 & 0.82 & & & & 0.73 \\
\hline 4.71 & 0.62 & & & 0.58 & 0.81 & & & & & 0.67 \\
\hline
\end{tabular}


Table 11 Normalized fluorescence intensities of ELMO-GFP and CherrySlam measured for Figure 21D.

\begin{tabular}{|c|c|c|c|c|c|c|c|c|}
\hline & \multicolumn{2}{|c|}{ Furrow 12 min } & \multicolumn{2}{|c|}{ Furrow $22 \mathrm{~min}$} & \multicolumn{2}{|c|}{ Furrow $32 \mathrm{~min}$} & \multicolumn{2}{|c|}{ Average $2 \mathrm{~min}$} \\
\hline $\begin{array}{l}\text { a-b } \\
\text { pos. }\end{array}$ & $\begin{array}{l}\text { ELM } \\
\text { O }\end{array}$ & Slam & $\begin{array}{l}\text { ELM } \\
\text { O }\end{array}$ & Slam & $\begin{array}{l}\text { ELM } \\
\text { O }\end{array}$ & Slam & $\begin{array}{l}\text { ELM } \\
\text { O }\end{array}$ & Slam \\
\hline$[\mu \mathrm{m}]$ & $\begin{array}{l}\text { Norm } \\
\text { [a.u.] }\end{array}$ & $\begin{array}{l}\text { Norm } \\
\text { [a.u.] }\end{array}$ & $\begin{array}{l}\text { Norm } \\
\text { [a.u.] }\end{array}$ & $\begin{array}{l}\text { Norm } \\
\text { [a.u.] }\end{array}$ & $\begin{array}{l}\text { Norm } \\
\text { [a.u.] }\end{array}$ & $\begin{array}{l}\text { Norm } \\
\text { [a.u.] }\end{array}$ & $\begin{array}{l}\text { Norm } \\
\text { [a.u.] }\end{array}$ & $\begin{array}{l}\text { Norm } \\
\text { [a.u.] }\end{array}$ \\
\hline 0.00 & 0.75 & & 0.82 & & 0.86 & & 0.75 & \\
\hline 0.50 & 0.69 & & 0.61 & & 0.75 & & 0.69 & \\
\hline 1.00 & 0.54 & 0.41 & 0.53 & 0.55 & 0.67 & 0.34 & 0.54 & 0.41 \\
\hline 1.50 & 0.51 & 0.32 & 0.49 & 0.44 & 0.60 & 0.36 & 0.51 & 0.32 \\
\hline 2.00 & 0.40 & 0.30 & 0.47 & 0.37 & 0.50 & 0.39 & 0.40 & 0.30 \\
\hline 2.50 & 0.31 & 0.23 & 0.32 & 0.27 & 0.40 & 0.30 & 0.31 & 0.23 \\
\hline 3.00 & 0.24 & 0.19 & 0.23 & 0.20 & 0.36 & 0.25 & 0.24 & 0.19 \\
\hline 3.50 & 0.20 & 0.17 & 0.18 & 0.16 & 0.30 & 0.20 & 0.20 & 0.17 \\
\hline 4.00 & 0.19 & 0.17 & 0.16 & 0.16 & 0.29 & 0.18 & 0.19 & 0.17 \\
\hline 4.50 & 0.18 & 0.15 & 0.13 & 0.15 & 0.28 & 0.21 & 0.18 & 0.15 \\
\hline 5.00 & 0.21 & 0.14 & 0.14 & 0.15 & 0.20 & 0.18 & 0.21 & 0.14 \\
\hline 5.50 & 0.20 & 0.15 & 0.13 & 0.14 & 0.25 & 0.17 & 0.20 & 0.15 \\
\hline 6.00 & 0.20 & 0.16 & 0.23 & 0.16 & 0.25 & 0.20 & 0.20 & 0.16 \\
\hline 6.50 & 0.22 & 0.20 & 0.23 & 0.19 & 0.27 & 0.23 & 0.22 & 0.20 \\
\hline 7.00 & 0.24 & 0.29 & 0.28 & 0.27 & 0.34 & 0.31 & 0.24 & 0.29 \\
\hline $\begin{array}{l}\text { Pos. } \\
{[\mu \mathrm{m}]}\end{array}$ & \multicolumn{2}{|c|}{ Furrow 16 min } & \multicolumn{2}{|c|}{ Furrow 26 min } & \multicolumn{2}{|c|}{ Furrow $36 \mathrm{~min}$} & \multicolumn{2}{|c|}{ Average $6 \mathrm{~min}$} \\
\hline 0.00 & 1.00 & & 0.91 & & 0.72 & & 1.00 & \\
\hline 0.50 & 0.94 & & 0.75 & & 0.63 & & 0.94 & \\
\hline 1.00 & 0.82 & 0.33 & 0.63 & 0.33 & 0.64 & 0.35 & 0.82 & 0.33 \\
\hline 1.50 & 0.76 & 0.27 & 0.63 & 0.27 & 0.66 & 0.32 & 0.76 & 0.27 \\
\hline 2.00 & 0.72 & 0.28 & 0.57 & 0.26 & 0.61 & 0.27 & 0.72 & 0.28 \\
\hline 2.50 & 0.67 & 0.26 & 0.48 & 0.29 & 0.63 & 0.33 & 0.67 & 0.26 \\
\hline 3.00 & 0.61 & 0.38 & 0.43 & 0.35 & 0.54 & 0.43 & 0.61 & 0.38 \\
\hline 3.50 & 0.59 & 0.58 & 0.42 & 0.47 & 0.45 & 0.44 & 0.59 & 0.58 \\
\hline 4.00 & 0.53 & 0.81 & 0.40 & 0.59 & 0.41 & 0.64 & 0.53 & 0.81 \\
\hline 4.50 & 0.48 & 0.92 & 0.39 & 0.68 & 0.40 & 0.78 & 0.48 & 0.92 \\
\hline 5.00 & 0.37 & 0.94 & 0.33 & 0.60 & 0.38 & 1.00 & 0.37 & 0.94 \\
\hline 5.50 & 0.30 & 0.53 & 0.26 & 0.41 & 0.31 & 0.63 & 0.30 & 0.53 \\
\hline 6.00 & 0.22 & 0.35 & 0.24 & 0.25 & 0.27 & 0.45 & 0.22 & 0.35 \\
\hline 6.50 & 0.25 & 0.29 & 0.20 & 0.23 & 0.25 & 0.34 & 0.25 & 0.29 \\
\hline 7.00 & 0.25 & 0.42 & 0.26 & 0.33 & 0.29 & 0.43 & 0.25 & 0.42 \\
\hline
\end{tabular}


Appendix

Table 12 Normalized fluorescence intensities of Armadillo signal for Figure 37C

\begin{tabular}{|c|c|c|c|c|c|c|c|c|c|c|}
\hline \multirow{3}{*}{$\begin{array}{l}\begin{array}{l}\text { a-b pos. } \\
{[\mu \mathrm{m}]}\end{array} \\
0.00\end{array}$} & \multicolumn{10}{|c|}{ Normalized fluorescence intensity of Arm signal [a.u.] wild type } \\
\hline & \multicolumn{3}{|c|}{ Embryo 1} & \multicolumn{3}{|c|}{ Embryo 2} & \multicolumn{3}{|c|}{ Embryo 3} & \multirow{2}{*}{$\begin{array}{r}\text { Average } \\
0.05\end{array}$} \\
\hline & 0.04 & & & & & & 0.07 & & & \\
\hline 0.12 & 0.06 & & & & & & 0.07 & 0.09 & & 0.07 \\
\hline 0.24 & 0.06 & & & & & & 0.06 & 0.14 & & 0.09 \\
\hline 0.36 & 0.08 & 0.02 & & 0.11 & & & 0.05 & 0.20 & 0.02 & 0.08 \\
\hline 0.48 & 0.09 & 0.03 & & 0.15 & & & 0.08 & 0.27 & 0.04 & 0.11 \\
\hline 0.60 & 0.07 & 0.04 & & 0.12 & & & 0.10 & 0.45 & 0.05 & 0.14 \\
\hline 0.72 & 0.07 & 0.04 & & 0.14 & 0.13 & & 0.08 & 0.46 & 0.06 & 0.14 \\
\hline 0.85 & 0.06 & 0.05 & & 0.16 & 0.13 & & 0.11 & 0.28 & 0.05 & 0.12 \\
\hline 0.97 & 0.07 & 0.09 & & 0.21 & 0.16 & & 0.20 & 0.14 & 0.05 & 0.13 \\
\hline 1.09 & 0.13 & 0.08 & & 0.17 & 0.20 & 0.15 & 0.24 & 0.10 & 0.06 & 0.14 \\
\hline 1.21 & 0.17 & 0.05 & & 0.12 & 0.26 & 0.26 & 0.19 & 0.09 & 0.05 & 0.15 \\
\hline 1.33 & 0.24 & 0.05 & & 0.16 & 0.29 & 0.11 & 0.16 & 0.07 & 0.06 & 0.14 \\
\hline 1.45 & 0.27 & 0.08 & & 0.20 & 0.29 & 0.09 & 0.17 & 0.07 & 0.06 & 0.15 \\
\hline 1.57 & 0.39 & 0.06 & & 0.21 & 0.27 & 0.11 & 0.18 & 0.08 & 0.07 & 0.17 \\
\hline 1.69 & 0.35 & 0.10 & & 0.19 & 0.24 & 0.09 & 0.18 & 0.11 & 0.08 & 0.17 \\
\hline 1.81 & 0.16 & 0.17 & & 0.20 & 0.29 & 0.12 & 0.34 & 0.13 & 0.09 & 0.19 \\
\hline 1.93 & 0.13 & 0.17 & 0.08 & 0.26 & 0.30 & 0.16 & 0.53 & 0.13 & 0.10 & 0.21 \\
\hline 2.05 & 0.14 & & 0.17 & & 0.51 & & 0.65 & 0.18 & 0.15 & 0.29 \\
\hline 2.17 & 0.20 & 0.34 & 0.23 & 0.46 & 0.75 & 0.23 & 0.76 & 0.31 & 0.23 & 0.39 \\
\hline 2.29 & 0.27 & 0.37 & 0.21 & 0.52 & 0.85 & 0.28 & 0.65 & 0.39 & 0.29 & 0.43 \\
\hline 2.41 & 0.24 & & 0.15 & 0.67 & 0.91 & & 0.50 & 0.45 & 0.36 & 0.43 \\
\hline 2.53 & 0.26 & 0.09 & 0.12 & 0.82 & 0.91 & 0.68 & 0.55 & 0.53 & 0.33 & 0.48 \\
\hline 2.66 & 0.33 & 0.10 & 0.13 & 0.91 & 0.90 & 0.73 & 0.66 & 0.50 & 0.33 & 0.51 \\
\hline 2.78 & 0.36 & 0.10 & 0.30 & 0.90 & 0.94 & 0.79 & 0.67 & 0.43 & 0.32 & 0.53 \\
\hline 2.90 & 0.62 & 0.21 & 0.58 & 0.95 & 0.91 & 0.75 & 0.88 & 0.38 & 0.36 & 0.63 \\
\hline 3.02 & 0.93 & 0.58 & 0.89 & 0.96 & 0.97 & 0.95 & 0.98 & 0.51 & 0.62 & 0.82 \\
\hline 3.14 & 1.00 & 1.00 & 0.90 & 0.97 & 1.00 & 1.00 & 1.00 & 0.63 & 0.75 & 0.92 \\
\hline 3.26 & 0.70 & 0.97 & 0.57 & 0.93 & 0.83 & 0.80 & 0.89 & 0.61 & 0.70 & 0.78 \\
\hline 3.38 & 0.62 & 0.70 & 0.33 & 0.69 & 0.51 & 0.78 & 0.58 & 0.55 & 0.58 & 0.59 \\
\hline 3.50 & 0.56 & 0.60 & 0.27 & 0.43 & 0.41 & 0.77 & 0.33 & 0.40 & 0.57 & 0.48 \\
\hline 3.62 & 0.49 & 0.60 & 0.25 & 0.41 & 0.35 & 0.59 & 0.20 & 0.28 & 0.41 & 0.40 \\
\hline 3.74 & 0.44 & 0.42 & 0.22 & 0.39 & 0.34 & 0.47 & 0.16 & 0.25 & 0.30 & 0.33 \\
\hline 3.86 & 0.33 & 0.35 & 0.24 & 0.33 & 0.38 & 0.38 & 0.16 & 0.22 & 0.25 & 0.29 \\
\hline 3.98 & 0.23 & 0.27 & 0.17 & 0.28 & 0.33 & 0.24 & 0.16 & 0.17 & 0.24 & 0.23 \\
\hline 4.10 & 0.18 & 0.15 & 0.15 & 0.16 & 0.25 & 0.18 & 0.13 & 0.14 & 0.26 & 0.18 \\
\hline 4.22 & 0.14 & 0.17 & 0.14 & 0.11 & 0.23 & 0.19 & 0.13 & 0.12 & 0.21 & 0.16 \\
\hline 4.35 & 0.13 & 0.38 & 0.19 & 0.11 & 0.29 & 0.23 & 0.13 & 0.15 & 0.20 & 0.20 \\
\hline 4.47 & 0.21 & 0.42 & 0.37 & 0.09 & 0.32 & 0.24 & 0.14 & 0.20 & 0.21 & 0.24 \\
\hline 4.59 & 0.33 & 0.36 & 0.56 & 0.09 & 0.24 & 0.17 & 0.11 & 0.15 & 0.27 & 0.25 \\
\hline 4.71 & 0.27 & 0.37 & 0.66 & 0.13 & 0.19 & 0.16 & 0.13 & 0.14 & 0.26 & 0.26 \\
\hline 4.83 & 0.22 & 0.30 & 0.75 & 0.14 & 0.13 & 0.17 & 0.15 & 0.16 & 0.19 & 0.24 \\
\hline 4.95 & 0.21 & 0.35 & 0.59 & 0.12 & 0.12 & 0.15 & 0.13 & 0.19 & 0.28 & 0.24 \\
\hline
\end{tabular}


Appendix

Normalized fluorescence intensities of Armadillo signal for Figure 37C - continuation

\begin{tabular}{|c|c|c|c|c|c|c|c|c|c|c|}
\hline \multirow{3}{*}{ 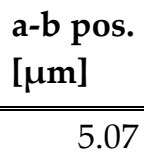 } & \multicolumn{10}{|c|}{ Normalized fluorescence intensity of Arm signal [a.u.] wild type } \\
\hline & \multicolumn{3}{|c|}{ Embryo 1} & \multicolumn{3}{|c|}{ Embryo 2} & \multicolumn{3}{|c|}{ Embryo 3} & \multirow{2}{*}{$\begin{array}{r}\text { Average } \\
0.23\end{array}$} \\
\hline & 0.14 & 0.44 & 0.45 & 0.10 & 0.11 & 0.16 & 0.13 & 0.23 & 0.32 & \\
\hline 5.19 & 0.09 & 0.31 & 0.34 & 0.11 & 0.11 & 0.16 & 0.13 & 0.26 & 0.22 & 0.19 \\
\hline 5.31 & 0.09 & 0.14 & 0.24 & 0.15 & 0.11 & 0.18 & 0.14 & 0.27 & 0.21 & 0.17 \\
\hline 5.43 & 0.11 & 0.08 & 0.26 & 0.15 & 0.10 & 0.22 & 0.17 & 0.26 & 0.26 & 0.18 \\
\hline 5.55 & 0.11 & 0.08 & 0.26 & 0.16 & 0.08 & 0.21 & 0.22 & 0.32 & 0.24 & 0.19 \\
\hline 5.67 & 0.16 & 0.11 & 0.25 & 0.16 & 0.09 & 0.21 & 0.32 & 0.32 & 0.19 & 0.20 \\
\hline 5.79 & 0.15 & 0.16 & 0.21 & 0.15 & 0.10 & 0.23 & 0.27 & 0.29 & 0.25 & 0.20 \\
\hline 5.91 & 0.10 & 0.19 & 0.18 & 0.15 & 0.12 & 0.28 & 0.18 & 0.30 & 0.22 & 0.19 \\
\hline 6.04 & 0.07 & 0.15 & 0.17 & 0.13 & 0.14 & 0.25 & 0.13 & 0.28 & 0.19 & 0.17 \\
\hline 6.16 & 0.07 & 0.10 & 0.20 & 0.10 & 0.14 & 0.20 & 0.19 & 0.27 & 0.15 & 0.16 \\
\hline 6.28 & 0.08 & 0.09 & 0.21 & 0.09 & 0.11 & 0.15 & 0.27 & 0.25 & 0.19 & 0.16 \\
\hline 6.40 & 0.06 & 0.10 & 0.25 & 0.07 & 0.09 & 0.11 & 0.28 & 0.25 & 0.21 & 0.16 \\
\hline 6.52 & 0.06 & 0.09 & 0.31 & 0.09 & 0.11 & 0.11 & 0.27 & 0.26 & 0.26 & 0.17 \\
\hline 6.64 & 0.08 & 0.07 & 0.34 & 0.14 & 0.13 & 0.13 & 0.15 & 0.25 & 0.30 & 0.18 \\
\hline 6.76 & 0.10 & 0.07 & 0.30 & 0.19 & 0.14 & 0.09 & 0.14 & 0.21 & 0.19 & 0.16 \\
\hline 6.88 & 0.11 & 0.09 & 0.19 & 0.30 & 0.17 & 0.09 & 0.18 & 0.22 & 0.16 & 0.17 \\
\hline 7.00 & 0.15 & 0.11 & 0.13 & 0.37 & 0.21 & 0.12 & 0.19 & 0.25 & 0.18 & 0.19 \\
\hline 7.12 & 0.17 & 0.09 & 0.13 & 0.27 & 0.22 & 0.12 & 0.23 & 0.32 & 0.19 & 0.19 \\
\hline 7.24 & 0.16 & 0.10 & 0.17 & 0.16 & 0.16 & 0.12 & 0.38 & 0.34 & 0.22 & 0.20 \\
\hline 7.36 & 0.20 & 0.10 & 0.22 & 0.13 & 0.16 & 0.10 & 0.44 & 0.26 & 0.23 & 0.21 \\
\hline 7.48 & 0.18 & 0.10 & 0.25 & 0.14 & 0.19 & 0.10 & 0.32 & 0.17 & 0.29 & 0.19 \\
\hline 7.60 & 0.14 & 0.15 & 0.25 & 0.17 & 0.18 & 0.12 & 0.25 & 0.14 & 0.24 & 0.18 \\
\hline 7.73 & 0.20 & 0.14 & 0.16 & 0.17 & 0.17 & 0.16 & 0.23 & 0.12 & 0.21 & 0.17 \\
\hline 7.85 & 0.26 & 0.08 & 0.13 & 0.16 & 0.16 & 0.24 & 0.27 & 0.17 & 0.23 & 0.19 \\
\hline 7.97 & 0.24 & 0.07 & 0.15 & 0.14 & 0.15 & 0.21 & 0.36 & 0.23 & 0.21 & 0.20 \\
\hline 8.09 & 0.20 & 0.09 & 0.17 & 0.11 & 0.14 & 0.18 & 0.42 & 0.26 & 0.32 & 0.21 \\
\hline 8.21 & 0.23 & 0.15 & 0.17 & 0.10 & 0.15 & 0.14 & 0.35 & 0.33 & 0.29 & 0.21 \\
\hline 8.33 & 0.23 & 0.19 & 0.23 & 0.14 & 0.16 & 0.10 & 0.28 & 0.39 & 0.24 & 0.22 \\
\hline 8.45 & 0.20 & 0.15 & 0.31 & 0.16 & 0.16 & 0.10 & 0.31 & 0.31 & 0.26 & 0.22 \\
\hline 8.57 & 0.19 & 0.16 & 0.30 & 0.12 & 0.18 & 0.12 & 0.31 & 0.23 & 0.26 & 0.21 \\
\hline 8.69 & 0.16 & 0.13 & 0.19 & 0.12 & 0.17 & 0.13 & 0.26 & 0.22 & 0.34 & 0.19 \\
\hline 8.81 & 0.19 & 0.09 & 0.14 & 0.14 & 0.12 & 0.26 & 0.22 & 0.23 & 0.50 & 0.21 \\
\hline 8.93 & 0.22 & 0.12 & 0.13 & 0.13 & 0.12 & 0.38 & 0.24 & 0.23 & 0.53 & 0.23 \\
\hline 9.05 & 0.19 & 0.13 & 0.14 & 0.15 & 0.17 & 0.37 & 0.23 & 0.24 & 0.40 & 0.22 \\
\hline 9.17 & 0.19 & 0.09 & 0.21 & 0.18 & 0.27 & 0.33 & 0.22 & 0.25 & 0.32 & 0.23 \\
\hline 9.29 & 0.24 & 0.07 & 0.32 & 0.27 & 0.33 & 0.25 & 0.29 & 0.24 & 0.26 & 0.25 \\
\hline 9.42 & 0.20 & 0.09 & 0.32 & 0.45 & 0.34 & 0.22 & 0.33 & 0.25 & 0.32 & 0.28 \\
\hline 9.54 & 0.14 & 0.07 & 0.25 & 0.58 & 0.47 & 0.22 & 0.28 & 0.30 & 0.50 & 0.31 \\
\hline 9.66 & 0.13 & 0.06 & 0.23 & 0.56 & 0.55 & 0.35 & 0.20 & 0.37 & 0.51 & 0.33 \\
\hline 9.78 & 0.15 & 0.09 & 0.26 & 0.55 & 0.45 & 0.39 & 0.20 & 0.41 & 0.62 & 0.35 \\
\hline 9.90 & 0.15 & 0.08 & 0.30 & 0.67 & 0.38 & 0.25 & 0.20 & 0.46 & 0.59 & 0.34 \\
\hline 10.02 & 0.13 & 0.10 & 0.29 & 0.82 & 0.41 & 0.22 & 0.27 & 0.54 & 0.53 & 0.37 \\
\hline 10.14 & 0.16 & 0.11 & 0.25 & 0.85 & 0.50 & 0.28 & 0.36 & 0.52 & 0.55 & 0.40 \\
\hline
\end{tabular}


Normalized fluorescence intensities of Armadillo signal for Figure 37C - continuation

\begin{tabular}{|c|c|c|c|c|c|c|c|c|c|c|}
\hline \multirow{3}{*}{ 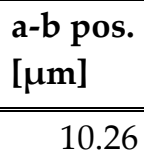 } & \multicolumn{10}{|c|}{ Normalized fluorescence intensitiy of Arm signal [a.u.] wild type } \\
\hline & \multicolumn{3}{|c|}{ Embryo 1} & \multicolumn{3}{|c|}{ Embryo 2} & \multicolumn{3}{|c|}{ Embryo 3} & \multirow{2}{*}{$\begin{array}{r}\text { Average } \\
0.40\end{array}$} \\
\hline & 0.17 & 0.10 & 0.30 & 0.91 & 0.50 & 0.38 & 0.35 & 0.43 & 0.49 & \\
\hline 10.38 & 0.18 & 0.08 & 0.30 & 1.00 & 0.52 & 0.51 & 0.39 & 0.41 & 0.45 & 0.43 \\
\hline 10.50 & 0.17 & 0.09 & 0.20 & 0.88 & 0.55 & 0.53 & 0.39 & 0.56 & 0.33 & 0.41 \\
\hline 10.62 & 0.16 & 0.09 & 0.18 & 0.58 & 0.53 & 0.50 & 0.27 & 0.67 & 0.36 & 0.37 \\
\hline 10.74 & 0.17 & 0.10 & 0.25 & 0.31 & 0.46 & 0.45 & 0.24 & 0.68 & 0.64 & 0.36 \\
\hline 10.86 & 0.21 & 0.17 & 0.33 & 0.16 & 0.42 & 0.43 & 0.18 & 0.61 & 1.00 & 0.39 \\
\hline 10.98 & 0.20 & 0.20 & 0.47 & 0.14 & 0.37 & 0.59 & 0.11 & 0.58 & 0.77 & 0.38 \\
\hline 11.11 & 0.19 & 0.22 & 0.57 & & 0.32 & 0.85 & & 0.57 & 0.47 & 0.45 \\
\hline 11.23 & 0.19 & 0.24 & 0.38 & & 0.26 & 0.92 & & 0.67 & 0.41 & 0.44 \\
\hline 11.35 & 0.18 & 0.21 & 0.25 & & & 0.81 & & 0.78 & 0.44 & 0.45 \\
\hline 11.47 & 0.15 & 0.22 & 0.24 & & & 0.55 & & 0.91 & 0.53 & 0.43 \\
\hline 11.59 & 0.12 & 0.17 & 0.27 & & & 0.32 & & 1.00 & 0.51 & 0.40 \\
\hline 11.71 & 0.12 & 0.13 & 0.24 & & & 0.19 & & 0.76 & 0.45 & 0.31 \\
\hline 11.83 & & 0.14 & 0.20 & & & 0.11 & & 0.47 & 0.52 & 0.29 \\
\hline 11.95 & & 0.16 & 0.21 & & & & & 0.32 & 0.49 & 0.29 \\
\hline 12.07 & & 0.29 & 0.24 & & & & & & & 0.27 \\
\hline \multirow{2}{*}{$\begin{array}{l}\text { a-b pos. } \\
{[\mu \mathrm{m}]}\end{array}$} & \multicolumn{10}{|c|}{ Normalized fluorescence intensitiy of Arm signal [a.u.] dia } \\
\hline & \multicolumn{3}{|c|}{ Embryo 1} & \multicolumn{3}{|c|}{ Embryo 2} & \multicolumn{3}{|c|}{ Embryo 3} & Average \\
\hline 0.00 & 0.13 & 0.19 & 0.11 & 0.11 & 0.08 & 0.09 & 0.09 & 0.12 & 0.14 & 0.12 \\
\hline 0.12 & 0.18 & 0.33 & 0.19 & 0.13 & 0.10 & 0.10 & 0.09 & 0.14 & 0.17 & 0.16 \\
\hline 0.24 & 0.23 & 0.21 & 0.15 & 0.12 & 0.08 & 0.09 & 0.12 & 0.18 & 0.18 & 0.15 \\
\hline 0.36 & 0.32 & 0.14 & 0.20 & 0.13 & 0.09 & 0.08 & 0.17 & 0.19 & 0.17 & 0.17 \\
\hline 0.48 & 0.32 & 0.13 & 0.14 & 0.13 & 0.09 & 0.08 & 0.15 & 0.20 & 0.16 & 0.16 \\
\hline 0.60 & 0.27 & 0.15 & 0.14 & 0.14 & 0.07 & 0.08 & 0.14 & 0.17 & 0.18 & 0.15 \\
\hline 0.72 & 0.27 & 0.27 & 0.09 & 0.13 & 0.06 & 0.09 & 0.11 & 0.18 & 0.23 & 0.16 \\
\hline 0.85 & 0.25 & 0.52 & 0.08 & 0.13 & 0.08 & 0.11 & 0.14 & 0.20 & 0.20 & 0.19 \\
\hline 0.97 & 0.35 & 0.88 & 0.09 & 0.17 & 0.08 & 0.13 & 0.15 & 0.18 & 0.12 & 0.24 \\
\hline 1.09 & 0.40 & 0.70 & 0.11 & 0.15 & 0.17 & 0.14 & 0.20 & 0.30 & 0.17 & 0.26 \\
\hline 1.21 & 0.40 & 0.28 & 0.18 & 0.17 & 0.20 & 0.19 & 0.28 & 0.51 & 0.17 & 0.26 \\
\hline 1.33 & 0.28 & 0.26 & 0.16 & 0.19 & 0.12 & 0.22 & 0.35 & 0.35 & 0.20 & 0.24 \\
\hline 1.45 & 0.19 & 0.30 & 0.16 & 0.16 & 0.11 & 0.20 & 0.38 & 0.26 & 0.23 & 0.22 \\
\hline 1.57 & 0.21 & 0.27 & 0.25 & 0.16 & 0.11 & 0.17 & 0.34 & 0.28 & 0.24 & 0.23 \\
\hline 1.69 & 0.28 & 0.24 & 0.31 & 0.19 & 0.11 & 0.16 & 0.39 & 0.37 & 0.23 & 0.25 \\
\hline 1.81 & 0.37 & 0.35 & 0.25 & 0.19 & 0.13 & 0.14 & 0.42 & 0.34 & 0.29 & 0.28 \\
\hline 1.93 & 0.42 & 0.47 & 0.21 & 0.17 & 0.12 & 0.13 & 0.33 & 0.32 & 0.32 & 0.28 \\
\hline 2.05 & 0.29 & 0.38 & 0.14 & 0.15 & 0.09 & 0.13 & 0.22 & 0.28 & 0.30 & 0.22 \\
\hline 2.17 & 0.19 & 0.21 & 0.17 & 0.19 & 0.06 & 0.13 & 0.21 & 0.30 & 0.23 & 0.19 \\
\hline 2.29 & 0.19 & 0.21 & 0.20 & 0.19 & 0.11 & 0.16 & 0.21 & 0.34 & 0.18 & 0.20 \\
\hline 2.41 & 0.18 & 0.18 & 0.14 & 0.18 & 0.15 & 0.15 & 0.21 & 0.47 & 0.15 & 0.20 \\
\hline 2.53 & 0.14 & 0.19 & 0.15 & 0.23 & 0.20 & 0.14 & 0.21 & 0.69 & 0.13 & 0.23 \\
\hline 2.66 & 0.13 & 0.37 & 0.13 & 0.28 & 0.19 & 0.14 & 0.22 & 0.65 & 0.12 & 0.25 \\
\hline 2.78 & 0.12 & 0.74 & 0.10 & 0.41 & 0.24 & 0.15 & 0.24 & 0.39 & 0.11 & 0.28 \\
\hline
\end{tabular}


Appendix

Normalized fluorescence intensities of Armadillo signal for Figure 37C - continuation

\begin{tabular}{|c|c|c|c|c|c|c|c|c|c|c|}
\hline \multirow{3}{*}{$\begin{array}{l}\begin{array}{l}\text { a-b pos. } \\
{[\mu \mathrm{m}]}\end{array} \\
2.90\end{array}$} & \multicolumn{10}{|c|}{ Normalized fluorescence intensity of Arm signal [a.u.] dia } \\
\hline & \multicolumn{3}{|c|}{ Embryo 1} & \multicolumn{3}{|c|}{ Embryo 2} & \multicolumn{3}{|c|}{ Embryo 3} & \multirow{2}{*}{$\begin{array}{r}\text { Average } \\
0.35\end{array}$} \\
\hline & 0.11 & 0.94 & 0.13 & 0.51 & 0.29 & 0.18 & 0.33 & 0.53 & 0.12 & \\
\hline 3.02 & 0.11 & 0.98 & 0.14 & 0.46 & 0.22 & 0.21 & 0.48 & 0.47 & 0.10 & 0.35 \\
\hline 3.14 & 0.12 & 1.00 & 0.18 & 0.30 & 0.19 & 0.23 & 0.55 & 0.25 & 0.10 & 0.33 \\
\hline 3.26 & 0.10 & 0.73 & 0.31 & 0.26 & 0.15 & 0.22 & 0.48 & 0.19 & 0.09 & 0.28 \\
\hline 3.38 & 0.13 & 0.22 & 0.31 & 0.33 & 0.16 & 0.18 & 0.56 & 0.22 & 0.08 & 0.24 \\
\hline 3.50 & 0.10 & 0.10 & 0.20 & 0.47 & 0.18 & 0.16 & 0.66 & 0.29 & 0.09 & 0.25 \\
\hline 3.62 & 0.10 & 0.15 & 0.35 & 0.60 & 0.22 & 0.13 & 0.49 & 0.36 & 0.09 & 0.28 \\
\hline 3.74 & 0.13 & 0.31 & 0.37 & 0.62 & 0.30 & 0.14 & 0.32 & 0.30 & 0.09 & 0.29 \\
\hline 3.86 & 0.14 & 0.23 & 0.18 & 0.46 & 0.37 & 0.14 & 0.26 & 0.28 & 0.09 & 0.24 \\
\hline 3.98 & 0.10 & 0.17 & 0.11 & 0.38 & 0.38 & 0.16 & 0.25 & 0.30 & 0.10 & 0.22 \\
\hline 4.10 & 0.08 & 0.17 & 0.16 & 0.52 & 0.31 & 0.24 & 0.35 & 0.31 & 0.13 & 0.25 \\
\hline 4.22 & 0.07 & 0.24 & 0.12 & 0.63 & 0.28 & 0.40 & 0.42 & 0.35 & 0.15 & 0.30 \\
\hline 4.35 & 0.06 & 0.25 & 0.19 & 0.59 & 0.37 & 0.48 & 0.36 & 0.36 & 0.14 & 0.31 \\
\hline 4.47 & 0.11 & 0.21 & 0.27 & 0.52 & 0.40 & 0.51 & 0.26 & 0.28 & 0.14 & 0.30 \\
\hline 4.59 & 0.14 & 0.33 & 0.27 & 0.47 & 0.41 & 0.44 & 0.19 & 0.28 & 0.13 & 0.30 \\
\hline 4.71 & 0.12 & 0.62 & 0.21 & & 0.40 & & & 0.22 & .12 & 0.30 \\
\hline 4.83 & 0.10 & 0.75 & 0.35 & 0.63 & 0.40 & 0.26 & 0.11 & 0.26 & 0.13 & 0.33 \\
\hline 4.95 & 0.09 & 0.56 & 0.37 & 0.67 & 0.38 & 0.25 & 0.11 & 0.24 & 0.16 & 0.31 \\
\hline 5.07 & 0.12 & 0.42 & & & 0.42 & & & 0.16 & .18 & 0.31 \\
\hline 5.19 & 0.18 & 0.44 & 0.46 & 0.73 & 0.44 & 0.38 & 0.10 & 0.10 & 0.15 & 0.33 \\
\hline 5.31 & 0.17 & 0.35 & 0.31 & 0.74 & 0.42 & 0.51 & 0.12 & 0.12 & 0.12 & 0.32 \\
\hline 5.43 & 0.19 & 0.29 & 0.15 & 0.71 & 0.34 & 0.69 & 0.14 & 0.16 & 0.12 & 0.31 \\
\hline 5.55 & 0.21 & 0.38 & 0.14 & 0.52 & 0.36 & 0.71 & 0.22 & 0.15 & 0.16 & 0.32 \\
\hline 5.67 & 0.16 & 0.28 & 0.30 & 0.35 & 0.32 & 0.58 & 0.31 & 0.15 & 0.29 & 0.30 \\
\hline 5.79 & 0.21 & 0.16 & 0.37 & 0.36 & 0.33 & 0.43 & 0.39 & 0.15 & 0.38 & 0.31 \\
\hline 5.91 & 0.21 & 0.14 & 0.25 & 0.40 & 0.35 & 0.29 & 0.41 & 0.18 & 0.27 & 0.28 \\
\hline 6.04 & 0.22 & 0.19 & 0.22 & 0.44 & 0.37 & 0.21 & 0.45 & 0.22 & 0.22 & 0.28 \\
\hline 6.16 & 0.17 & 0.40 & 0.40 & 0.47 & 0.38 & 0.19 & 0.53 & 0.30 & 0.20 & 0.34 \\
\hline 6.28 & 0.15 & 0.49 & 0.38 & 0.47 & 0.37 & 0.24 & 0.54 & 0.34 & 0.25 & 0.36 \\
\hline 6.40 & 0.12 & 0.60 & 0.35 & 0.40 & 0.32 & 0.36 & 0.56 & 0.39 & 0.27 & 0.37 \\
\hline 6.52 & 0.16 & 0.58 & 0.58 & 0.40 & 0.32 & 0.45 & 0.70 & 0.37 & 0.25 & 0.42 \\
\hline 6.64 & 0.18 & 0.38 & 0.92 & 0.43 & 0.30 & 0.54 & 0.65 & 0.36 & 0.30 & 0.45 \\
\hline 6.76 & 0.22 & 0.58 & 1.00 & 0.44 & 0.30 & 0.63 & 0.46 & 0.45 & 0.47 & 0.51 \\
\hline 6.88 & 0.26 & 0.71 & 0.74 & 0.45 & 0.33 & 0.62 & 0.38 & 0.60 & 0.56 & 0.52 \\
\hline 7.00 & 0.30 & 0.41 & 0.63 & 0.52 & 0.40 & 0.58 & 0.35 & 0.79 & 0.39 & 0.49 \\
\hline 7.12 & 0.30 & 0.21 & 0.75 & 0.60 & 0.47 & 0.59 & 0.41 & 0.85 & 0.21 & 0.49 \\
\hline 7.24 & 0.31 & 0.17 & 0.81 & 0.56 & 0.46 & 0.52 & 0.52 & 0.82 & 0.21 & 0.49 \\
\hline 7.36 & 0.27 & 0.18 & 0.78 & 0.47 & 0.49 & 0.46 & 0.55 & 0.59 & 0.21 & 0.44 \\
\hline 7.48 & 0.36 & 0.18 & 0.57 & 0.43 & 0.49 & 0.63 & 0.51 & 0.39 & 0.21 & 0.42 \\
\hline 7.60 & 0.53 & 0.15 & 0.52 & 0.51 & 0.46 & 0.71 & 0.40 & 0.44 & 0.30 & 0.45 \\
\hline 7.73 & 0.63 & 0.21 & 0.53 & 0.55 & 0.45 & 0.52 & 0.36 & 0.64 & 0.43 & 0.48 \\
\hline 7.85 & 0.61 & 0.38 & 0.56 & 0.49 & 0.40 & 0.33 & 0.34 & 0.81 & 0.44 & 0.48 \\
\hline 7.97 & 0.48 & 0.58 & 0.62 & 0.41 & 0.43 & 0.24 & 0.34 & 0.79 & 0.34 & 0.47 \\
\hline
\end{tabular}


Appendix

Normalized fluorescence intensities of Armadillo signal for Figure 37C - continuation

\begin{tabular}{|c|c|c|c|c|c|c|c|c|c|c|}
\hline \multirow{3}{*}{ 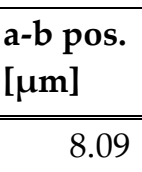 } & \multicolumn{10}{|c|}{ Normalized fluorescence intensity of Arm signal [a.u.] dia } \\
\hline & \multicolumn{3}{|c|}{ Embryo 1} & \multicolumn{3}{|c|}{ Embryo 2} & \multicolumn{3}{|c|}{ Embryo 3} & \multirow{2}{*}{$\begin{array}{r}\text { Average } \\
0.44\end{array}$} \\
\hline & 0.40 & 0.36 & 0.66 & 0.37 & 0.50 & 0.25 & 0.33 & 0.75 & 0.36 & \\
\hline 8.21 & 0.49 & 0.23 & 0.66 & 0.28 & 0.57 & 0.36 & 0.35 & 0.72 & 0.41 & 0.45 \\
\hline 8.33 & 0.55 & 0.19 & 0.70 & 0.20 & 0.51 & 0.34 & 0.42 & 0.93 & 0.39 & 0.47 \\
\hline 8.45 & 0.56 & 0.17 & 0.51 & 0.19 & 0.41 & 0.26 & 0.58 & 1.00 & 0.29 & 0.44 \\
\hline 8.57 & 0.55 & 0.16 & 0.60 & 0.24 & 0.40 & 0.22 & 0.72 & 0.96 & 0.19 & 0.45 \\
\hline 8.69 & 0.54 & 0.19 & 0.51 & 0.33 & 0.30 & 0.24 & 0.72 & 0.94 & 0.15 & 0.43 \\
\hline 8.81 & 0.39 & 0.19 & 0.45 & 0.44 & 0.23 & 0.27 & 0.70 & 0.80 & 0.13 & 0.40 \\
\hline 8.93 & 0.25 & 0.21 & 0.65 & 0.59 & 0.21 & 0.26 & 0.72 & 0.72 & 0.15 & 0.42 \\
\hline 9.05 & 0.35 & 0.18 & & 0.67 & 0.20 & 0.25 & 0.78 & 0.55 & 0.18 & 0.39 \\
\hline 9.17 & 0.45 & 0.20 & & 0.51 & 0.24 & 0.24 & 1.00 & 0.35 & 0.21 & 0.40 \\
\hline 9.29 & 0.53 & 0.34 & & 0.34 & 0.33 & 0.23 & 0.98 & 0.18 & 0.22 & 0.39 \\
\hline 9.42 & 0.58 & 0.47 & & 0.25 & 0.39 & 0.22 & 0.82 & 0.12 & 0.20 & 0.38 \\
\hline 9.54 & 0.48 & 0.48 & & 0.28 & 0.31 & 0.20 & 0.67 & 0.08 & 0.16 & 0.33 \\
\hline 9.66 & 0.33 & 0.60 & & 0.37 & 0.33 & 0.20 & 0.63 & 0.08 & 0.12 & 0.33 \\
\hline 9.78 & 0.26 & 0.32 & & 0.43 & 0.38 & 0.20 & 0.68 & & 0.13 & 0.34 \\
\hline 9.90 & 0.24 & 0.33 & & 0.44 & 0.45 & 0.20 & 0.72 & & 0.19 & 0.37 \\
\hline 10.02 & 0.23 & & & 0.34 & 0.42 & 0.21 & 0.66 & & 0.34 & 0.37 \\
\hline 10.14 & 0.27 & & & 0.26 & 0.33 & 0.24 & 0.51 & & 0.50 & 0.35 \\
\hline 10.26 & 0.35 & & & 0.29 & 0.29 & 0.30 & 0.40 & & 0.78 & 0.40 \\
\hline 10.38 & 0.51 & & & 0.39 & 0.28 & 0.38 & & & 1.00 & 0.51 \\
\hline 10.50 & 0.72 & & & 0.46 & 0.34 & 0.49 & & & 0.92 & 0.59 \\
\hline 10.62 & 0.75 & & & 0.46 & 0.40 & 0.56 & & & 0.66 & 0.56 \\
\hline 10.74 & 0.70 & & & 0.53 & 0.38 & 0.53 & & & 0.44 & 0.51 \\
\hline 10.86 & 0.92 & & & 0.57 & 0.35 & 0.48 & & & 0.38 & 0.54 \\
\hline 10.98 & 1.00 & & & 0.56 & 0.28 & 0.52 & & & 0.31 & 0.54 \\
\hline 11.11 & 0.78 & & & 0.47 & 0.29 & 0.53 & & & 0.22 & 0.46 \\
\hline 11.23 & & & & 0.44 & 0.27 & 0.59 & & & 0.15 & 0.36 \\
\hline 11.35 & & & & 0.43 & 0.22 & 0.72 & & & 0.16 & 0.38 \\
\hline 11.47 & & & & 0.48 & 0.24 & 0.83 & & & 0.17 & 0.43 \\
\hline 11.59 & & & & 0.53 & 0.25 & 0.89 & & & 0.14 & 0.45 \\
\hline 11.71 & & & & 0.58 & 0.29 & 0.92 & & & & 0.60 \\
\hline 11.83 & & & & 0.61 & 0.37 & 1.00 & & & & 0.66 \\
\hline 11.95 & & & & 0.59 & 0.55 & 1.00 & & & & 0.71 \\
\hline 12.07 & & & & 0.57 & 0.73 & & & & & 0.65 \\
\hline
\end{tabular}


Table 13 Normalized fluorescence intensities of Baz signal measured for Figure 38C

\begin{tabular}{|c|c|c|c|c|c|c|c|c|c|c|}
\hline \multirow{3}{*}{$\begin{array}{l}\begin{array}{l}\text { a-b pos. } \\
{[\mu \mathrm{m}]}\end{array} \\
\\
0.00\end{array}$} & \multicolumn{10}{|c|}{ Normalized fluorescence intensity of Baz signal [a.u.] wild type } \\
\hline & \multicolumn{3}{|c|}{ Embryo 1} & \multicolumn{3}{|c|}{ Embryo 2} & \multicolumn{3}{|c|}{ Embryo 3} & \multirow{2}{*}{$\begin{array}{r}\text { Average } \\
0.10\end{array}$} \\
\hline & 0.05 & 0.11 & & & & & 0.15 & & & \\
\hline 0.12 & 0.04 & 0.11 & & & & & 0.13 & & & 0.09 \\
\hline 0.24 & 0.06 & 0.15 & & & & & 0.21 & & & 0.14 \\
\hline 0.36 & 0.08 & 0.17 & 0.07 & & & & 0.23 & & & 0.14 \\
\hline 0.48 & 0.06 & 0.13 & 0.06 & & & & 0.19 & 0.11 & & 0.11 \\
\hline 0.60 & 0.03 & 0.10 & 0.06 & 0.11 & & & 0.32 & 0.17 & & 0.13 \\
\hline 0.72 & 0.04 & 0.11 & 0.07 & 0.09 & & & 0.41 & 0.20 & & 0.15 \\
\hline 0.85 & 0.03 & 0.16 & 0.07 & 0.11 & & & 0.27 & 0.21 & & 0.14 \\
\hline 0.97 & 0.04 & 0.20 & 0.06 & 0.10 & 0.05 & 0.18 & 0.29 & 0.18 & & 0.14 \\
\hline 1.09 & 0.06 & 0.22 & 0.07 & 0.12 & 0.07 & 0.18 & 0.27 & 0.24 & & 0.15 \\
\hline 1.21 & 0.10 & 0.22 & 0.08 & 0.18 & 0.08 & 0.27 & 0.36 & 0.28 & 0.31 & 0.21 \\
\hline 1.33 & 0.16 & 0.20 & 0.11 & 0.13 & 0.11 & 0.20 & 0.32 & 0.29 & 0.30 & 0.20 \\
\hline 1.45 & 0.22 & 0.20 & 0.12 & 0.18 & 0.12 & 0.28 & 0.30 & 0.29 & 0.31 & 0.22 \\
\hline 1.57 & 0.25 & 0.23 & 0.15 & 0.12 & 0.13 & 0.47 & 0.30 & 0.26 & 0.34 & 0.25 \\
\hline 1.69 & 0.15 & 0.22 & 0.16 & 0.09 & 0.13 & 0.36 & 0.25 & 0.22 & 0.39 & 0.22 \\
\hline 1.81 & 0.08 & 0.29 & 0.16 & 0.12 & 0.20 & 0.23 & 0.32 & 0.22 & 0.28 & 0.21 \\
\hline 1.93 & 0.08 & 0.29 & 0.14 & 0.10 & 0.14 & 0.28 & 0.44 & 0.19 & 0.26 & 0.21 \\
\hline 2.05 & 0.11 & 0.39 & 0.11 & 0.11 & 0.17 & 0.31 & 0.45 & 0.14 & 0.27 & 0.23 \\
\hline 2.17 & 0.14 & 0.77 & 0.14 & 0.16 & 0.18 & 0.34 & 0.34 & 0.13 & 0.24 & 0.27 \\
\hline 2.29 & 0.15 & 0.81 & 0.14 & 0.26 & 0.22 & 0.46 & 0.53 & 0.12 & 0.28 & 0.33 \\
\hline 2.41 & 0.14 & 0.89 & 0.13 & 0.30 & 0.18 & 0.38 & 0.72 & 0.20 & 0.25 & 0.35 \\
\hline 2.53 & 0.14 & 0.96 & 0.19 & 0.36 & 0.19 & 0.45 & 0.79 & 0.29 & 0.33 & 0.41 \\
\hline 2.66 & 0.15 & 0.90 & 0.24 & 0.37 & 0.20 & 0.58 & 0.70 & 0.41 & 0.29 & 0.43 \\
\hline 2.78 & 0.30 & 0.85 & 0.20 & 0.42 & 0.26 & 0.52 & 0.87 & 0.61 & 0.38 & 0.49 \\
\hline 2.90 & 0.51 & 0.69 & 0.25 & 0.41 & 0.26 & 0.52 & 0.78 & 0.73 & 0.52 & 0.52 \\
\hline 3.02 & 0.52 & 0.74 & 0.28 & 0.40 & 0.38 & 0.77 & 0.86 & 0.66 & 0.59 & 0.58 \\
\hline 3.14 & 0.55 & 0.70 & 0.48 & 0.48 & 0.53 & 0.76 & 0.83 & 0.50 & 0.73 & 0.62 \\
\hline 3.26 & 0.64 & 0.76 & 0.80 & 0.58 & 0.71 & 0.70 & 0.86 & 0.59 & 0.90 & 0.73 \\
\hline 3.38 & 0.72 & 0.97 & 0.99 & 0.90 & 0.86 & 0.83 & 0.97 & 0.80 & 0.88 & 0.88 \\
\hline 3.50 & 1.00 & 1.00 & 1.00 & 1.00 & 1.00 & 1.00 & 1.00 & 1.00 & 1.00 & 1.00 \\
\hline 3.62 & 0.85 & 0.92 & 0.72 & 0.81 & 0.74 & 0.74 & 0.89 & 0.72 & 0.74 & 0.79 \\
\hline 3.74 & 0.46 & 0.80 & 0.37 & 0.61 & 0.59 & 0.46 & 0.87 & 0.50 & 0.83 & 0.61 \\
\hline 3.86 & 0.27 & 0.64 & 0.25 & 0.45 & 0.52 & 0.37 & 0.64 & 0.33 & 0.65 & 0.46 \\
\hline 3.98 & 0.13 & 0.46 & 0.17 & 0.33 & 0.44 & 0.47 & 0.43 & 0.29 & 0.75 & 0.39 \\
\hline 4.10 & 0.11 & 0.43 & 0.14 & 0.25 & 0.34 & 0.32 & 0.30 & 0.26 & 0.73 & 0.32 \\
\hline 4.22 & 0.10 & 0.37 & 0.11 & 0.28 & 0.34 & 0.31 & 0.38 & 0.21 & 0.58 & 0.30 \\
\hline 4.35 & 0.13 & 0.26 & 0.08 & 0.18 & 0.28 & 0.35 & 0.31 & 0.19 & 0.81 & 0.29 \\
\hline 4.47 & 0.12 & 0.23 & 0.08 & 0.16 & 0.24 & 0.38 & 0.32 & 0.19 & 0.73 & 0.27 \\
\hline 4.59 & 0.10 & 0.22 & 0.09 & 0.14 & 0.20 & 0.46 & 0.34 & 0.14 & 0.80 & 0.28 \\
\hline 4.71 & 0.11 & 0.25 & 0.14 & 0.18 & 0.19 & 0.61 & 0.43 & 0.14 & 0.65 & 0.30 \\
\hline 4.83 & 0.14 & 0.17 & 0.21 & 0.19 & 0.19 & 0.44 & 0.40 & 0.19 & 0.55 & 0.28 \\
\hline 4.95 & 0.21 & 0.16 & 0.22 & 0.18 & 0.16 & 0.37 & 0.35 & 0.20 & 0.47 & 0.26 \\
\hline
\end{tabular}


Normalized fluorescence intensities of Baz signal for Figure 37C - continuation

\begin{tabular}{|c|c|c|c|c|c|c|c|c|c|c|}
\hline \multirow{3}{*}{$\begin{array}{l}\text { a-b pos. } \\
\text { [ } \mu \mathrm{m}]\end{array}$} & \multicolumn{10}{|c|}{ Normalized fluorescence intensity of Arm signal [a.u.] wild type } \\
\hline & \multicolumn{3}{|c|}{ Embryo 1} & \multicolumn{3}{|c|}{ Embryo 2} & \multicolumn{3}{|c|}{ Embryo 3} & \multirow{2}{*}{$\begin{array}{r}\text { Average } \\
0.27\end{array}$} \\
\hline & 0.24 & 0.13 & 0.18 & 0.20 & 0.13 & 0.38 & 0.42 & 0.19 & 0.54 & \\
\hline 5.19 & 0.17 & 0.14 & 0.16 & 0.17 & 0.13 & 0.28 & 0.43 & 0.19 & 0.61 & 0.25 \\
\hline 5.31 & 0.16 & 0.19 & 0.16 & 0.11 & 0.15 & 0.34 & 0.48 & 0.13 & 0.49 & 0.25 \\
\hline 5.43 & 0.10 & 0.19 & 0.13 & 0.10 & 0.14 & 0.41 & 0.45 & 0.16 & 0.49 & 0.24 \\
\hline 5.55 & 0.07 & 0.19 & 0.09 & 0.09 & 0.16 & 0.47 & 0.41 & 0.15 & 0.63 & 0.25 \\
\hline 5.67 & 0.08 & 0.23 & 0.10 & 0.10 & 0.13 & 0.54 & 0.37 & 0.13 & 0.40 & 0.23 \\
\hline 5.79 & 0.06 & 0.29 & 0.15 & 0.14 & 0.11 & 0.54 & 0.35 & 0.12 & 0.42 & 0.24 \\
\hline 5.91 & 0.09 & 0.38 & 0.21 & 0.12 & 0.10 & 0.40 & 0.26 & 0.14 & 0.42 & 0.24 \\
\hline 6.04 & 0.11 & 0.49 & 0.25 & 0.09 & 0.08 & 0.32 & 0.42 & 0.18 & 0.40 & 0.26 \\
\hline 6.16 & 0.11 & 0.49 & 0.27 & 0.08 & 0.07 & 0.30 & 0.52 & 0.18 & 0.41 & 0.27 \\
\hline 6.28 & 0.07 & 0.36 & 0.26 & 0.08 & 0.10 & 0.32 & 0.50 & 0.16 & 0.42 & 0.25 \\
\hline 6.40 & 0.07 & 0.27 & 0.22 & 0.08 & 0.10 & 0.24 & 0.57 & 0.17 & 0.34 & 0.23 \\
\hline 6.52 & 0.10 & 0.21 & 0.13 & 0.08 & 0.10 & 0.17 & 0.51 & 0.14 & 0.38 & 0.20 \\
\hline 6.64 & 0.06 & 0.20 & 0.12 & 0.10 & 0.11 & 0.19 & 0.31 & 0.11 & 0.36 & 0.17 \\
\hline 6.76 & 0.07 & 0.21 & 0.16 & 0.12 & 0.07 & 0.27 & 0.36 & 0.13 & 0.43 & 0.20 \\
\hline 6.88 & 0.08 & 0.21 & 0.25 & 0.12 & 0.09 & 0.26 & 0.30 & 0.16 & 0.38 & 0.20 \\
\hline 7.00 & 0.11 & 0.22 & 0.26 & 0.13 & 0.10 & 0.21 & 0.30 & 0.17 & 0.37 & 0.21 \\
\hline 7.12 & 0.12 & 0.13 & 0.19 & 0.11 & 0.08 & 0.17 & 0.29 & 0.15 & 0.32 & 0.17 \\
\hline 7.24 & 0.10 & 0.11 & 0.15 & 0.09 & 0.05 & 0.26 & 0.25 & 0.17 & 0.38 & 0.17 \\
\hline 7.36 & 0.07 & 0.10 & 0.12 & 0.06 & 0.06 & 0.25 & 0.30 & 0.15 & 0.38 & 0.17 \\
\hline 7.48 & 0.08 & 0.08 & 0.10 & 0.08 & 0.10 & 0.19 & 0.29 & 0.15 & 0.35 & 0.16 \\
\hline 7.60 & 0.10 & 0.11 & 0.07 & 0.09 & 0.09 & 0.22 & 0.30 & 0.14 & 0.37 & 0.17 \\
\hline 7.73 & 0.09 & 0.15 & 0.07 & 0.07 & 0.09 & 0.23 & 0.28 & 0.14 & 0.37 & 0.16 \\
\hline 7.85 & 0.07 & 0.15 & 0.07 & 0.07 & 0.07 & 0.23 & 0.28 & 0.13 & 0.41 & 0.16 \\
\hline 7.97 & 0.06 & 0.13 & 0.08 & 0.06 & 0.08 & 0.19 & 0.27 & 0.12 & 0.32 & 0.15 \\
\hline 8.09 & 0.04 & 0.10 & 0.11 & 0.08 & 0.07 & 0.31 & 0.37 & 0.16 & 0.32 & 0.17 \\
\hline 8.21 & 0.05 & 0.09 & 0.12 & 0.10 & 0.09 & 0.15 & 0.30 & 0.17 & 0.35 & 0.16 \\
\hline 8.33 & 0.04 & 0.11 & 0.12 & 0.09 & 0.10 & 0.15 & 0.38 & 0.09 & 0.52 & 0.18 \\
\hline 8.45 & 0.05 & 0.11 & 0.13 & 0.06 & 0.08 & 0.20 & 0.25 & 0.10 & 0.58 & 0.17 \\
\hline 8.57 & 0.06 & 0.11 & 0.14 & 0.05 & 0.13 & 0.12 & 0.21 & 0.11 & 0.58 & 0.17 \\
\hline 8.69 & 0.08 & 0.15 & 0.13 & 0.07 & 0.10 & 0.10 & 0.29 & 0.13 & 0.49 & 0.17 \\
\hline 8.81 & 0.09 & 0.14 & 0.12 & 0.06 & 0.08 & 0.12 & 0.38 & 0.12 & 0.35 & 0.16 \\
\hline 8.93 & 0.10 & 0.16 & 0.16 & 0.09 & 0.12 & 0.15 & 0.28 & 0.11 & 0.33 & 0.17 \\
\hline 9.05 & 0.09 & 0.19 & 0.17 & 0.10 & 0.13 & 0.17 & 0.28 & 0.13 & 0.27 & 0.17 \\
\hline 9.17 & 0.06 & 0.16 & 0.15 & 0.09 & 0.07 & 0.19 & 0.21 & 0.13 & 0.41 & 0.17 \\
\hline 9.29 & 0.07 & 0.09 & 0.16 & 0.10 & 0.06 & 0.23 & 0.18 & 0.11 & 0.31 & 0.15 \\
\hline 9.42 & 0.09 & 0.13 & 0.14 & 0.07 & 0.07 & 0.24 & 0.20 & 0.10 & 0.25 & 0.14 \\
\hline 9.54 & 0.08 & 0.13 & 0.12 & 0.07 & 0.08 & 0.26 & 0.13 & 0.12 & 0.33 & 0.15 \\
\hline 9.66 & 0.08 & 0.20 & 0.16 & 0.11 & 0.08 & 0.24 & 0.16 & 0.11 & 0.34 & 0.17 \\
\hline 9.78 & 0.08 & 0.19 & 0.16 & 0.11 & 0.09 & 0.18 & 0.17 & 0.14 & 0.26 & 0.15 \\
\hline 9.90 & 0.06 & 0.23 & 0.16 & 0.11 & 0.11 & 0.17 & 0.28 & 0.14 & 0.29 & 0.17 \\
\hline 10.02 & 0.06 & 0.23 & 0.13 & 0.11 & 0.14 & 0.20 & 0.32 & 0.13 & 0.43 & 0.20 \\
\hline 10.14 & 0.06 & 0.15 & 0.13 & 0.13 & 0.14 & 0.16 & 0.28 & 0.13 & 0.41 & 0.17 \\
\hline
\end{tabular}


Appendix

Normalized fluorescence intensities of Armadillo signal for Figure 37C - continuation

\begin{tabular}{|c|c|c|c|c|c|c|c|c|c|c|}
\hline \multirow{3}{*}{$\begin{array}{l}\begin{array}{l}\text { a-b pos. } \\
{[\mu \mathrm{m}]}\end{array} \\
\frac{10.26}{}\end{array}$} & \multicolumn{10}{|c|}{ Normalized fluorescence intensity of Baz signal [a.u.] wild type } \\
\hline & \multicolumn{3}{|c|}{ Embryo 1} & \multicolumn{3}{|c|}{ Embryo 2} & \multicolumn{3}{|c|}{ Embryo 3} & \multirow{2}{*}{$\begin{array}{r}\text { Average } \\
0.19 \\
\end{array}$} \\
\hline & 0.07 & 0.16 & 0.16 & 0.18 & 0.12 & 0.17 & 0.25 & 0.13 & 0.51 & \\
\hline 10.38 & 0.09 & 0.12 & 0.21 & 0.21 & 0.10 & 0.22 & 0.28 & 0.16 & 0.52 & 0.21 \\
\hline 10.50 & 0.09 & 0.14 & 0.16 & 0.16 & 0.09 & 0.18 & 0.33 & 0.18 & 0.29 & 0.18 \\
\hline 10.62 & 0.07 & 0.13 & 0.10 & 0.10 & 0.07 & 0.18 & 0.30 & 0.16 & 0.37 & 0.17 \\
\hline 10.74 & 0.09 & 0.21 & 0.10 & 0.11 & 0.11 & 0.23 & 0.26 & 0.12 & 0.47 & 0.19 \\
\hline 10.86 & 0.09 & 0.15 & 0.11 & 0.12 & 0.08 & 0.22 & 0.35 & 0.13 & 0.37 & 0.18 \\
\hline 10.98 & 0.10 & 0.16 & 0.12 & 0.14 & 0.06 & 0.23 & 0.28 & 0.10 & 0.36 & 0.17 \\
\hline 11.11 & 0.11 & 0.17 & 0.11 & 0.12 & 0.09 & 0.19 & 0.27 & 0.12 & 0.30 & 0.17 \\
\hline 11.23 & 0.11 & 0.22 & 0.08 & 0.11 & 0.11 & 0.11 & & 0.13 & 0.29 & 0.14 \\
\hline 11.35 & 0.10 & 0.26 & 0.09 & & 0.12 & 0.11 & & 0.15 & 0.26 & 0.16 \\
\hline 11.47 & 0.09 & 0.21 & 0.10 & & 0.10 & 0.17 & & 0.14 & 0.34 & 0.17 \\
\hline 11.59 & 0.09 & 0.26 & 0.13 & & & 0.25 & & 0.15 & 0.28 & 0.19 \\
\hline 11.71 & 0.09 & 0.24 & 0.18 & & & 0.24 & & 0.12 & 0.36 & 0.20 \\
\hline 11.83 & & 0.24 & 0.18 & & & & & 0.12 & 0.25 & 0.20 \\
\hline 11.95 & & 0.22 & 0.17 & & & & & 0.10 & 0.35 & 0.21 \\
\hline 12.07 & & 0.20 & 0.12 & & & & & 0.06 & 0.52 & 0.23 \\
\hline \multirow{2}{*}{$\begin{array}{l}\text { a-b pos. } \\
{[\mu \mathrm{m}]}\end{array}$} & \multicolumn{10}{|c|}{ Normalized fluorescence intensity of Baz signal [a.u.] dia } \\
\hline & \multicolumn{3}{|c|}{ Embryo 1} & \multicolumn{3}{|c|}{ Embryo 2} & \multicolumn{3}{|c|}{ Embryo 3} & Average \\
\hline 0.00 & 0.42 & 0.28 & 0.76 & 0.37 & 0.37 & 0.22 & 0.33 & 0.35 & 0.29 & 0.38 \\
\hline 0.12 & 0.39 & 0.32 & 0.86 & 0.41 & 0.32 & 0.29 & 0.35 & 0.34 & 0.38 & 0.41 \\
\hline 0.24 & 0.55 & 0.26 & 0.70 & 0.48 & 0.37 & 0.34 & 0.38 & 0.37 & 0.39 & 0.43 \\
\hline 0.36 & 0.54 & 0.28 & 0.80 & 0.42 & 0.37 & 0.39 & 0.43 & 0.39 & 0.44 & 0.45 \\
\hline 0.48 & 0.67 & 0.30 & 0.72 & 0.51 & 0.47 & 0.37 & 0.46 & 0.44 & 0.44 & 0.49 \\
\hline 0.60 & 0.68 & 0.34 & 0.63 & 0.45 & 0.42 & 0.41 & 0.50 & 0.45 & 0.45 & 0.48 \\
\hline 0.72 & 0.69 & 0.51 & 0.61 & 0.49 & 0.45 & 0.41 & 0.43 & 0.49 & 0.46 & 0.50 \\
\hline 0.85 & 0.72 & 0.54 & 0.73 & 0.44 & 0.58 & 0.45 & 0.43 & 0.53 & 0.50 & 0.55 \\
\hline 0.97 & 0.72 & 0.58 & 0.83 & 0.38 & 0.59 & 0.46 & 0.46 & 0.52 & 0.51 & 0.56 \\
\hline 1.09 & 0.73 & 0.47 & 0.80 & 0.41 & 0.55 & 0.42 & 0.47 & 0.61 & 0.53 & 0.55 \\
\hline 1.21 & 0.73 & 0.39 & 0.89 & 0.34 & 0.48 & 0.47 & 0.46 & 0.76 & 0.55 & 0.56 \\
\hline 1.33 & 0.77 & 0.34 & 0.99 & 0.33 & 0.61 & 0.55 & 0.57 & 0.79 & 0.47 & 0.60 \\
\hline 1.45 & 0.74 & 0.35 & 1.00 & 0.34 & 0.58 & 0.51 & 0.58 & 0.79 & 0.42 & 0.59 \\
\hline 1.57 & 0.89 & 0.39 & 0.88 & 0.49 & 0.56 & 0.63 & 0.48 & 0.76 & 0.50 & 0.62 \\
\hline 1.69 & 0.76 & 0.38 & 0.94 & 0.55 & 0.60 & 0.72 & 0.50 & 0.62 & 0.41 & 0.61 \\
\hline 1.81 & 0.86 & 0.40 & 0.83 & 0.61 & 0.62 & 0.64 & 0.49 & 0.62 & 0.40 & 0.61 \\
\hline 1.93 & 0.92 & 0.42 & 0.75 & 0.55 & 0.65 & 0.62 & 0.46 & 0.60 & 0.47 & 0.60 \\
\hline 2.05 & 0.86 & 0.45 & 0.74 & 0.53 & 0.67 & 0.58 & 0.42 & 0.69 & 0.53 & 0.61 \\
\hline 2.17 & 0.76 & 0.40 & 0.52 & 0.56 & 0.60 & 0.54 & 0.39 & 0.71 & 0.63 & 0.57 \\
\hline 2.29 & 0.73 & 0.40 & 0.59 & 0.58 & 0.70 & 0.62 & 0.49 & 0.68 & 0.67 & 0.61 \\
\hline 2.41 & 0.68 & 0.45 & 0.44 & 0.55 & 0.73 & 0.58 & 0.58 & 0.68 & 0.65 & 0.59 \\
\hline 2.53 & 0.52 & 0.51 & 0.60 & 0.52 & 0.61 & 0.63 & 0.57 & 0.74 & 0.69 & 0.60 \\
\hline 2.66 & 0.52 & 0.65 & 0.59 & 0.52 & 0.60 & 0.65 & 0.52 & 0.72 & 0.59 & 0.59 \\
\hline 2.78 & 0.68 & 0.75 & 0.64 & 0.59 & 0.53 & 0.65 & 0.58 & 0.60 & 0.49 & 0.61 \\
\hline
\end{tabular}


Normalized fluorescence intensities of Armadillo signal for Figure 37C - continuation

\begin{tabular}{|c|c|c|c|c|c|c|c|c|c|c|}
\hline \multirow{3}{*}{$\begin{array}{l}\begin{array}{l}\text { a-b pos. } \\
{[\mu \mathrm{m}]}\end{array} \\
2.90\end{array}$} & \multicolumn{10}{|c|}{ Normalized fluorescence intensity of Baz signal [a.u.] dia } \\
\hline & \multicolumn{3}{|c|}{ Embryo 1} & \multicolumn{3}{|c|}{ Embryo 2} & \multicolumn{3}{|c|}{ Embryo 3} & \multirow{2}{*}{$\begin{array}{r}\text { Average } \\
0.64\end{array}$} \\
\hline & 0.71 & 0.93 & 0.74 & 0.57 & 0.60 & 0.62 & 0.58 & 0.66 & 0.36 & \\
\hline 3.02 & 0.66 & 1.00 & 0.74 & 0.50 & 0.55 & 0.59 & 0.62 & 0.74 & 0.31 & 0.63 \\
\hline 3.14 & 0.57 & 0.90 & 0.75 & 0.48 & 0.60 & 0.60 & 0.59 & 0.52 & 0.23 & 0.58 \\
\hline 3.26 & 0.57 & 0.66 & 0.76 & 0.46 & 0.57 & 0.53 & 0.62 & 0.54 & 0.28 & 0.56 \\
\hline 3.38 & 0.43 & 0.55 & 0.77 & 0.47 & 0.67 & 0.51 & 0.59 & 0.44 & 0.25 & 0.52 \\
\hline 3.50 & 0.48 & 0.38 & 0.83 & 0.47 & 0.63 & 0.70 & 0.54 & 0.44 & 0.19 & 0.52 \\
\hline 3.62 & 0.57 & 0.32 & 0.73 & 0.41 & 0.63 & 0.69 & 0.58 & 0.32 & 0.25 & 0.50 \\
\hline 3.74 & 0.68 & 0.31 & 0.65 & 0.43 & 0.65 & 0.80 & 0.51 & 0.36 & 0.26 & 0.52 \\
\hline 3.86 & 0.64 & 0.31 & 0.64 & 0.52 & 0.64 & 0.90 & 0.57 & 0.50 & 0.22 & 0.55 \\
\hline 3.98 & 0.49 & 0.37 & 0.61 & 0.48 & 0.55 & 1.00 & 0.53 & 0.44 & 0.22 & 0.52 \\
\hline 4.10 & 0.47 & 0.35 & 0.76 & 0.48 & 0.70 & 0.91 & 0.53 & 0.51 & 0.24 & 0.55 \\
\hline 4.22 & 0.50 & 0.37 & 0.78 & 0.60 & 0.70 & 0.80 & 0.54 & 0.37 & 0.34 & 0.55 \\
\hline 4.35 & 0.66 & 0.36 & 0.74 & 0.65 & 0.66 & 0.76 & 0.57 & 0.42 & 0.30 & 0.57 \\
\hline 4.47 & 0.57 & 0.42 & 0.70 & 0.58 & 0.71 & 0.85 & 0.50 & 0.37 & 0.28 & 0.55 \\
\hline 4.59 & 0.48 & 0.35 & 0.74 & 0.70 & 0.60 & 0.83 & 0.50 & 0.40 & 0.32 & 0.55 \\
\hline 4.71 & 0.44 & 0.37 & 0.58 & 0.70 & 0.61 & 0.88 & & 0.40 & 0.38 & 0.54 \\
\hline 4.83 & 0.41 & 0.42 & 0.55 & 0.84 & 0.76 & 0.97 & 0.40 & 0.43 & 0.40 & 0.58 \\
\hline 4.95 & 0.40 & 0.34 & 0.55 & 1.00 & 0.68 & 0.98 & 0.42 & 0.39 & 0.45 & 0.58 \\
\hline 5.07 & 0.43 & 0.26 & 0.64 & 0.85 & 0.73 & 0.98 & & 0.44 & 0.42 & 0.58 \\
\hline 5.19 & 0.33 & 0.25 & 0.66 & 0.73 & 0.86 & 0.96 & 0.46 & 0.56 & 0.45 & 0.59 \\
\hline 5.31 & 0.40 & 0.28 & 0.60 & 0.65 & 0.94 & 0.89 & 0.50 & 0.62 & 0.41 & 0.59 \\
\hline 5.43 & 0.48 & 0.26 & 0.57 & 0.57 & 1.00 & 0.97 & 0.53 & 0.61 & 0.45 & 0.60 \\
\hline 5.55 & 0.60 & 0.25 & 0.52 & 0.58 & 0.86 & 0.89 & 0.76 & 0.71 & 0.39 & 0.62 \\
\hline 5.67 & 0.51 & 0.25 & 0.60 & 0.66 & 0.76 & 1.00 & 0.83 & 0.77 & 0.45 & 0.65 \\
\hline 5.79 & 0.61 & 0.28 & 0.50 & 0.73 & 0.62 & 0.98 & 0.81 & 0.73 & 0.31 & 0.62 \\
\hline 5.91 & 0.60 & 0.37 & 0.48 & 0.74 & 0.72 & 0.80 & 0.78 & 0.84 & 0.29 & 0.62 \\
\hline 6.04 & 0.64 & 0.41 & 0.59 & 0.76 & 0.65 & 0.64 & 0.66 & 0.79 & 0.38 & 0.61 \\
\hline 6.16 & 0.62 & 0.46 & 0.55 & 0.89 & 0.74 & 0.66 & 0.67 & 0.85 & 0.43 & 0.65 \\
\hline 6.28 & 0.57 & 0.59 & 0.62 & 0.95 & 0.81 & 0.72 & 0.51 & 0.96 & 0.40 & 0.68 \\
\hline 6.40 & 0.53 & 0.72 & 0.61 & 0.82 & 0.80 & 0.72 & 0.50 & 1.00 & 0.31 & 0.67 \\
\hline 6.52 & 0.61 & 0.83 & 0.63 & 0.76 & 0.72 & 0.78 & 0.47 & 0.75 & 0.25 & 0.65 \\
\hline 6.64 & 0.56 & 0.70 & 0.70 & 0.75 & 0.78 & 0.88 & 0.47 & 0.71 & 0.40 & 0.66 \\
\hline 6.76 & 0.55 & 0.62 & 0.59 & 0.71 & 0.96 & 0.90 & 0.44 & 0.67 & 0.30 & 0.64 \\
\hline 6.88 & 0.46 & 0.59 & 0.63 & 0.60 & 0.86 & 0.86 & 0.40 & 0.64 & 0.30 & 0.59 \\
\hline 7.00 & 0.45 & 0.57 & 0.69 & 0.53 & 0.74 & 0.84 & 0.33 & 0.73 & 0.28 & 0.57 \\
\hline 7.12 & 0.52 & 0.53 & 0.69 & 0.44 & 0.67 & 0.76 & 0.37 & 0.65 & 0.26 & 0.54 \\
\hline 7.24 & 0.61 & 0.39 & 0.66 & 0.47 & 0.74 & 0.67 & 0.37 & 0.66 & 0.19 & 0.53 \\
\hline 7.36 & 0.70 & 0.38 & 0.53 & 0.55 & 0.65 & 0.58 & 0.40 & 0.65 & 0.16 & 0.51 \\
\hline 7.48 & 0.79 & 0.37 & 0.45 & 0.53 & 0.72 & 0.61 & 0.46 & 0.75 & 0.18 & 0.54 \\
\hline 7.60 & 0.88 & 0.33 & 0.45 & 0.55 & 0.70 & 0.54 & 0.42 & 0.89 & 0.20 & 0.55 \\
\hline 7.73 & 1.00 & 0.31 & 0.57 & 0.57 & 0.58 & 0.55 & 0.37 & 0.94 & 0.22 & 0.57 \\
\hline 7.85 & 0.96 & 0.32 & 0.58 & 0.47 & 0.69 & 0.54 & 0.45 & 0.79 & 0.27 & 0.56 \\
\hline 7.97 & 0.95 & 0.32 & 0.59 & 0.36 & 0.70 & 0.57 & 0.42 & 0.59 & 0.32 & 0.53 \\
\hline
\end{tabular}


Appendix

Normalized fluorescence intensities of Armadillo signal for Figure 37C - continuation

\begin{tabular}{|c|c|c|c|c|c|c|c|c|c|c|}
\hline \multirow{3}{*}{$\begin{array}{l}\begin{array}{l}\text { a-b pos. } \\
{[\mu \mathrm{m}]}\end{array} \\
8.09\end{array}$} & \multicolumn{10}{|c|}{ Normalized fluorescence intensity of Baz signal [a.u.] dia } \\
\hline & \multicolumn{3}{|c|}{ Embryo 1} & \multicolumn{3}{|c|}{ Embryo 2} & \multicolumn{3}{|c|}{ Embryo 3} & \multirow{2}{*}{$\begin{array}{r}\text { Average } \\
0.52\end{array}$} \\
\hline & 0.87 & 0.35 & 0.53 & 0.38 & 0.68 & 0.61 & 0.33 & 0.63 & 0.30 & \\
\hline 8.21 & 0.81 & 0.38 & 0.52 & 0.39 & 0.65 & 0.61 & 0.36 & 0.48 & 0.25 & 0.49 \\
\hline 8.33 & 0.94 & 0.35 & 0.54 & 0.36 & 0.68 & 0.53 & 0.38 & 0.60 & 0.18 & 0.51 \\
\hline 8.45 & 0.88 & 0.40 & 0.52 & 0.41 & 0.78 & 0.49 & 0.54 & 0.51 & 0.17 & 0.52 \\
\hline 8.57 & 0.70 & 0.32 & 0.65 & 0.47 & 0.76 & 0.52 & 0.74 & 0.46 & 0.18 & 0.53 \\
\hline 8.69 & 0.60 & 0.28 & 0.65 & 0.51 & 0.82 & 0.42 & 1.00 & 0.40 & 0.17 & 0.54 \\
\hline 8.81 & 0.51 & 0.36 & 0.77 & 0.60 & 0.78 & 0.49 & 1.00 & 0.40 & 0.19 & 0.57 \\
\hline 8.93 & 0.40 & 0.36 & 0.70 & 0.67 & 0.77 & 0.55 & 0.89 & 0.37 & 0.24 & 0.55 \\
\hline 9.05 & 0.35 & 0.32 & & 0.62 & 0.69 & 0.38 & 0.82 & 0.56 & 0.27 & 0.50 \\
\hline 9.17 & 0.41 & 0.26 & & 0.52 & 0.62 & 0.35 & 0.99 & 0.47 & 0.24 & 0.48 \\
\hline 9.29 & 0.39 & 0.32 & & 0.49 & 0.76 & 0.39 & 0.92 & 0.30 & 0.27 & 0.48 \\
\hline 9.42 & 0.34 & 0.36 & & 0.44 & 0.74 & 0.46 & 0.81 & 0.36 & 0.35 & 0.48 \\
\hline 9.54 & 0.41 & 0.39 & & 0.44 & 0.83 & 0.55 & 0.72 & 0.45 & 0.32 & 0.51 \\
\hline 9.66 & 0.45 & 0.33 & & 0.46 & 0.76 & 0.44 & 0.53 & 0.38 & 0.38 & 0.47 \\
\hline 9.78 & 0.39 & 0.30 & & 0.45 & 0.72 & 0.45 & 0.42 & & 0.45 & 0.45 \\
\hline 9.90 & 0.39 & 0.24 & & 0.39 & 0.71 & 0.48 & 0.40 & & 0.62 & 0.46 \\
\hline 10.02 & 0.42 & & & 0.43 & 0.74 & 0.46 & 0.35 & & 0.95 & 0.56 \\
\hline 10.14 & 0.45 & & & 0.45 & 0.64 & 0.47 & 0.37 & & 1.00 & 0.56 \\
\hline 10.26 & 0.49 & & & 0.46 & 0.64 & 0.56 & 0.37 & & 0.70 & 0.53 \\
\hline 10.38 & 0.52 & & & 0.51 & 0.61 & 0.59 & & & 0.51 & 0.55 \\
\hline 10.50 & 0.51 & & & 0.54 & 0.55 & 0.54 & & & 0.35 & 0.50 \\
\hline 10.62 & 0.58 & & & 0.47 & 0.66 & 0.57 & & & 0.29 & 0.52 \\
\hline 10.74 & 0.63 & & & 0.48 & 0.72 & 0.53 & & & 0.31 & 0.53 \\
\hline 10.86 & 0.57 & & & 0.44 & 0.61 & 0.53 & & & 0.28 & 0.49 \\
\hline 10.98 & 0.48 & & & 0.39 & 0.67 & 0.50 & & & 0.25 & 0.46 \\
\hline 11.11 & 0.51 & & & 0.37 & 0.56 & 0.54 & & & 0.24 & 0.45 \\
\hline 11.23 & & & & 0.39 & 0.54 & 0.57 & & & 0.20 & 0.42 \\
\hline 11.35 & & & & 0.49 & 0.50 & 0.65 & & & 0.25 & 0.47 \\
\hline 11.47 & & & & 0.50 & 0.64 & 0.65 & & & 0.26 & 0.51 \\
\hline 11.59 & & & & 0.58 & 0.64 & 0.49 & & & & 0.57 \\
\hline 11.71 & & & & 0.56 & 0.74 & 0.54 & & & & 0.61 \\
\hline 11.83 & & & & 0.58 & 0.76 & 0.51 & & & & 0.62 \\
\hline 11.95 & & & & 0.53 & 0.67 & 0.43 & & & & 0.54 \\
\hline 12.07 & & & & 0.51 & 0.69 & & & & & 0.60 \\
\hline
\end{tabular}


Table 14 Normalized fluorescence intensity of Cno signal measured for Figure 40C.

\begin{tabular}{|c|c|c|c|c|c|c|c|c|c|c|}
\hline \multirow{3}{*}{$\begin{array}{l}\begin{array}{l}\text { a-b pos. } \\
{[\mu \mathrm{m}]}\end{array} \\
\\
\end{array}$} & \multicolumn{10}{|c|}{ Normalized fluorescence intensity of Cno signal [a.u.] dia } \\
\hline & \multicolumn{3}{|c|}{ Embryo 1} & \multicolumn{3}{|c|}{ Embryo 2} & \multicolumn{3}{|c|}{ Embryo 3} & \multirow{2}{*}{$\begin{array}{r}\text { Averag } \\
0.2\end{array}$} \\
\hline & & & & & & & 0.26 & & & \\
\hline 0.19 & 0.17 & & & & & & 0.32 & & & 0.24 \\
\hline 0.38 & 0.29 & & 0.09 & & & & 0.31 & 0.28 & 0.10 & 0.21 \\
\hline 0.56 & 0.23 & 0.05 & 0.22 & & & & 0.41 & 0.50 & 0.18 & 0.26 \\
\hline 0.75 & 0.25 & 0.10 & 0.39 & & & 0.16 & 0.49 & 0.53 & 0.26 & 0.31 \\
\hline 0.94 & 0.36 & 0.15 & 0.58 & & & 0.31 & 0.49 & 0.48 & 0.34 & 0.39 \\
\hline 1.13 & 0.60 & 0.23 & 0.46 & 0.11 & 0.22 & 0.37 & 0.56 & 0.62 & 0.46 & 0.40 \\
\hline 1.32 & 0.74 & 0.33 & 0.64 & 0.36 & 0.37 & 0.55 & 0.49 & 0.71 & 0.53 & 0.52 \\
\hline 1.51 & 0.86 & 0.51 & 0.77 & 0.77 & 0.80 & 0.80 & 0.73 & 0.88 & 0.67 & 0.76 \\
\hline 1.69 & 1.00 & 1.00 & 1.00 & 0.91 & 1.00 & 1.00 & 1.00 & 1.00 & 1.00 & 0.99 \\
\hline 1.88 & 0.92 & 0.94 & 0.73 & 0.97 & 0.98 & 0.67 & 0.78 & 0.69 & 0.52 & 0.80 \\
\hline 2.07 & 0.69 & 0.71 & 0.76 & 1.00 & 0.95 & 0.52 & 0.69 & 0.60 & 0.38 & 0.70 \\
\hline 2.26 & 0.56 & 0.52 & 0.75 & 0.63 & 0.93 & 0.34 & 0.67 & 0.49 & 0.31 & 0.58 \\
\hline 2.45 & 0.46 & 0.51 & 0.74 & 0.39 & 0.66 & 0.23 & 0.70 & 0.43 & 0.20 & 0.48 \\
\hline 2.64 & 0.48 & 0.38 & 0.54 & 0.24 & 0.37 & 0.15 & 0.53 & 0.43 & 0.18 & 0.37 \\
\hline 2.82 & 0.18 & 0.30 & 0.15 & 0.16 & 0.23 & 0.15 & 0.25 & 0.38 & 0.21 & 0.22 \\
\hline 3.01 & 0.09 & 0.25 & 0.13 & 0.08 & 0.16 & 0.14 & 0.17 & 0.31 & 0.16 & 0.17 \\
\hline 3.20 & 0.06 & 0.21 & 0.28 & 0.05 & 0.15 & 0.13 & 0.17 & 0.23 & 0.13 & 0.16 \\
\hline 3.39 & 0.14 & 0.13 & 0.57 & 0.05 & 0.11 & 0.13 & 0.19 & 0.18 & 0.15 & 0.18 \\
\hline 3.58 & 0.24 & 0.07 & 0.33 & 0.03 & 0.09 & 0.10 & 0.18 & 0.19 & 0.14 & 0.15 \\
\hline 3.77 & 0.18 & 0.07 & 0.20 & 0.05 & 0.11 & 0.10 & 0.14 & 0.19 & 0.12 & 0.13 \\
\hline 3.95 & 0.16 & 0.09 & 0.14 & 0.04 & 0.06 & 0.07 & 0.14 & 0.19 & 0.15 & 0.11 \\
\hline 4.14 & 0.08 & 0.12 & 0.14 & 0.02 & 0.06 & 0.08 & 0.18 & 0.19 & 0.14 & 0.11 \\
\hline 4.33 & 0.04 & 0.12 & 0.06 & 0.03 & 0.05 & 0.12 & 0.20 & 0.20 & 0.13 & 0.11 \\
\hline 4.52 & 0.12 & 0.12 & 0.04 & 0.05 & 0.07 & 0.12 & 0.17 & 0.19 & 0.15 & 0.11 \\
\hline 4.71 & 0.15 & 0.09 & 0.04 & 0.03 & 0.07 & 0.08 & 0.17 & 0.19 & 0.14 & 0.11 \\
\hline 4.89 & 0.07 & 0.06 & 0.10 & 0.02 & 0.05 & 0.15 & 0.16 & 0.16 & 0.13 & 0.10 \\
\hline 5.08 & 0.07 & 0.05 & 0.10 & 0.00 & 0.05 & 0.14 & 0.17 & 0.16 & 0.11 & 0.09 \\
\hline 5.27 & 0.06 & 0.05 & 0.12 & 0.01 & 0.09 & 0.11 & 0.21 & 0.18 & 0.10 & 0.10 \\
\hline 5.46 & 0.08 & 0.03 & 0.18 & 0.03 & 0.05 & 0.11 & 0.20 & 0.14 & 0.09 & 0.10 \\
\hline 5.65 & 0.10 & 0.03 & 0.23 & 0.03 & 0.03 & 0.07 & 0.17 & 0.13 & 0.09 & 0.10 \\
\hline 5.84 & 0.13 & 0.04 & 0.32 & 0.03 & 0.04 & 0.08 & 0.15 & 0.14 & 0.11 & 0.11 \\
\hline 6.02 & 0.22 & 0.10 & 0.19 & 0.02 & 0.04 & 0.08 & 0.15 & 0.12 & 0.11 & 0.11 \\
\hline 6.21 & 0.24 & 0.09 & 0.13 & 0.01 & 0.11 & 0.06 & 0.18 & 0.11 & 0.12 & 0.12 \\
\hline 6.40 & 0.19 & 0.08 & 0.09 & 0.03 & 0.05 & 0.10 & 0.22 & 0.16 & 0.11 & 0.11 \\
\hline 6.59 & 0.19 & 0.05 & 0.14 & 0.02 & 0.05 & 0.09 & 0.19 & 0.21 & 0.12 & 0.12 \\
\hline 6.78 & 0.20 & 0.09 & 0.11 & 0.01 & 0.03 & 0.07 & 0.14 & 0.23 & 0.15 & 0.11 \\
\hline 6.97 & 0.15 & 0.07 & 0.15 & 0.01 & 0.01 & 0.14 & & 0.18 & 0.18 & 0.11 \\
\hline 7.15 & 0.13 & 0.07 & 0.16 & 0.02 & 0.03 & 0.24 & & 0.13 & 0.19 & 0.12 \\
\hline 7.34 & & 0.09 & 0.18 & 0.05 & 0.08 & & & & 0.22 & 0.12 \\
\hline 7.53 & & 0.05 & & 0.11 & 0.08 & & & & 0.21 & 0.11 \\
\hline 7.72 & & & & 0.11 & 0.09 & & & & & 0.10 \\
\hline
\end{tabular}


Appendix

Normalized fluorescence intensities of Cno signal for Figure 40C - continuation

\begin{tabular}{|c|c|c|c|c|c|c|c|c|c|c|}
\hline \multirow{3}{*}{$\begin{array}{l}7.91 \\
\text { a-b pos. } \\
{[\mu \mathrm{m}]}\end{array}$} & & & & 0.13 & 0.15 & & & & & 0.14 \\
\hline & \multicolumn{10}{|c|}{ Normalized fluorescence intensity of Cno signal [a.u.] dia } \\
\hline & \multicolumn{3}{|c|}{ Embryo 1} & \multicolumn{3}{|c|}{ Embryo 2} & \multicolumn{3}{|c|}{ Embryo 3} & Average \\
\hline 0.00 & 0.58 & & & 0.41 & & & & & & 0.50 \\
\hline 0.19 & 0.76 & & & 0.51 & 0.50 & & & & & 0.59 \\
\hline 0.38 & 0.63 & & & 0.66 & 0.57 & & & & & 0.62 \\
\hline 0.56 & 0.63 & & & 0.56 & 0.64 & & & & & 0.61 \\
\hline 0.75 & 0.54 & & & 0.59 & 0.66 & & & & & 0.59 \\
\hline 0.94 & 0.50 & 0.30 & 0.53 & 0.62 & 0.75 & & & & 0.14 & 0.47 \\
\hline 1.13 & 0.56 & 0.62 & 0.77 & 0.56 & 0.75 & 0.22 & 0.34 & 0.48 & 0.27 & 0.51 \\
\hline 1.32 & 0.65 & 0.79 & 0.72 & 0.63 & 0.79 & 0.47 & 0.51 & 0.61 & 0.59 & 0.64 \\
\hline 1.51 & 0.80 & 0.86 & 0.79 & 0.85 & 0.89 & 0.78 & 0.84 & 0.83 & 0.85 & 0.83 \\
\hline 1.69 & 1.00 & 1.00 & 0.85 & 1.00 & 0.91 & 0.91 & 1.00 & 1.00 & 0.98 & 0.96 \\
\hline 1.88 & 0.94 & 0.97 & 0.85 & 0.88 & 0.99 & 0.97 & 0.82 & 0.85 & 1.00 & 0.92 \\
\hline 2.07 & 0.75 & 0.83 & 0.73 & 0.56 & 0.88 & 1.00 & 0.72 & 0.60 & 0.98 & 0.78 \\
\hline 2.26 & 0.57 & 0.80 & 0.79 & 0.42 & 0.85 & 0.98 & 0.75 & 0.55 & 0.99 & 0.74 \\
\hline 2.45 & 0.45 & 0.81 & 0.75 & 0.41 & 0.87 & 0.76 & 0.66 & 0.51 & 0.93 & 0.68 \\
\hline 2.64 & 0.42 & 0.74 & 0.65 & 0.38 & 0.44 & 0.49 & 0.52 & 0.42 & 0.82 & 0.54 \\
\hline 2.82 & 0.50 & 0.60 & 0.64 & 0.41 & 0.34 & 0.38 & 0.57 & 0.48 & 0.73 & 0.52 \\
\hline 3.01 & 0.37 & 0.56 & 0.76 & 0.41 & 0.32 & 0.59 & 0.61 & 0.57 & 0.67 & 0.54 \\
\hline 3.20 & 0.40 & 0.65 & 0.71 & 0.40 & 0.25 & 0.93 & 0.49 & 0.58 & 0.76 & 0.57 \\
\hline 3.39 & 0.42 & 0.79 & 0.67 & 0.33 & 0.29 & 0.92 & 0.42 & 0.56 & 0.81 & 0.58 \\
\hline 3.58 & 0.40 & 0.48 & 0.70 & 0.32 & 0.55 & 0.65 & 0.40 & 0.63 & 0.79 & 0.55 \\
\hline 3.77 & 0.33 & 0.29 & 0.63 & 0.44 & 0.74 & 0.54 & 0.36 & 0.79 & 0.74 & 0.54 \\
\hline 3.95 & 0.26 & 0.45 & 0.53 & 0.45 & 0.82 & 0.46 & 0.34 & 0.69 & 0.67 & 0.52 \\
\hline 4.14 & 0.24 & 0.72 & 0.53 & 0.40 & 1.00 & 0.42 & 0.35 & 0.57 & 0.77 & 0.56 \\
\hline 4.33 & 0.24 & 0.71 & 0.58 & 0.35 & 0.92 & 0.31 & 0.39 & 0.48 & 0.58 & 0.51 \\
\hline 4.52 & 0.28 & 0.49 & 0.49 & 0.31 & 0.49 & 0.24 & 0.42 & 0.45 & 0.47 & 0.40 \\
\hline 4.71 & 0.33 & 0.40 & 0.41 & 0.23 & 0.35 & 0.20 & 0.39 & 0.46 & 0.47 & 0.36 \\
\hline 4.89 & 0.38 & 0.38 & 0.41 & 0.18 & 0.38 & 0.23 & 0.40 & 0.39 & 0.40 & 0.35 \\
\hline 5.08 & 0.33 & 0.30 & 0.39 & 0.20 & 0.33 & 0.19 & 0.39 & 0.37 & 0.33 & 0.31 \\
\hline 5.27 & 0.25 & 0.25 & 0.56 & 0.24 & 0.34 & 0.25 & 0.37 & 0.40 & 0.31 & 0.33 \\
\hline 5.46 & 0.29 & 0.26 & 0.83 & 0.35 & 0.47 & 0.26 & 0.38 & 0.41 & 0.30 & 0.39 \\
\hline 5.65 & 0.31 & 0.35 & 1.00 & 0.39 & 0.68 & 0.21 & 0.39 & 0.36 & 0.28 & 0.44 \\
\hline 5.84 & 0.26 & 0.53 & 0.90 & & 0.49 & 0.17 & 0.47 & 0.31 & 0.29 & 0.43 \\
\hline 6.02 & 0.35 & 0.57 & 0.69 & & 0.36 & 0.18 & 0.58 & 0.31 & 0.28 & 0.42 \\
\hline 6.21 & 0.53 & 0.28 & 0.58 & & 0.27 & 0.22 & 0.57 & 0.34 & 0.32 & 0.39 \\
\hline 6.40 & 0.55 & 0.17 & 0.58 & & 0.25 & 0.30 & 0.50 & 0.41 & 0.44 & 0.40 \\
\hline 6.59 & 0.54 & 0.18 & 0.51 & & & 0.21 & 0.42 & 0.46 & 0.42 & 0.39 \\
\hline 6.78 & & 0.14 & 0.38 & & & 0.15 & 0.43 & 0.51 & 0.30 & 0.32 \\
\hline 6.97 & & 0.15 & 0.38 & & & 0.16 & 0.53 & 0.48 & 0.33 & 0.34 \\
\hline 7.15 & & 0.17 & 0.46 & & & 0.23 & 0.53 & 0.49 & & 0.38 \\
\hline 7.34 & & 0.16 & 0.56 & & & 0.27 & & 0.50 & & 0.37 \\
\hline 7.53 & & & 0.73 & & & 0.28 & & & & 0.50 \\
\hline
\end{tabular}


Normalized fluorescence intensities of Cno signal for Figure 40C - continuation

\begin{tabular}{|c|c|c|c|c|c|c|}
\hline \multirow{2}{*}{$\begin{array}{l}\text { a-b pos. } \\
{[\mu \mathrm{m}]}\end{array}$} & \multicolumn{6}{|c|}{ Normalized fluorescence intensity of Cno signal [a.u.] dia } \\
\hline & Embryo 1 & & Embryo & & Embryo 3 & Average \\
\hline 7.72 & & 0.82 & & 0.29 & & 0.55 \\
\hline 7.91 & & 0.81 & & 0.27 & & 0.54 \\
\hline
\end{tabular}


Table 15 Normalized fluorescence intensities of Spg singal measured for Figure 41C.

\begin{tabular}{|c|c|c|c|c|c|c|c|c|c|c|c|c|c|c|c|c|c|c|c|c|}
\hline \multirow{3}{*}{$\begin{array}{l}\begin{array}{l}\text { a-b } \\
\text { pos. }[\mu \mathrm{m}] \\
-1.69\end{array}\end{array}$} & \multicolumn{20}{|c|}{ Normalized fluorescence intensities of Spg signal [a.u.] wild type } \\
\hline & \multicolumn{4}{|c|}{ Embryo 1} & \multicolumn{5}{|c|}{ Embryo 2} & \multicolumn{5}{|c|}{ Embryo 3} & \multicolumn{5}{|c|}{ Embryo 4} & \multirow{2}{*}{$\begin{array}{r}\text { Average } \\
0.49\end{array}$} \\
\hline & 0.47 & & & & & 0.65 & & & & & & & 0.33 & & & & 0.72 & & 0.26 & \\
\hline-1.51 & 0.59 & & & & & 0.66 & & & & & & & 0.36 & & & & 0.77 & & 0.40 & 0.55 \\
\hline-1.13 & 0.86 & & 0.49 & 0.19 & & 0.78 & 0.33 & & & & & & 0.64 & & & & 0.88 & & 0.52 & 0.58 \\
\hline-0.94 & 0.80 & 0.50 & 0.67 & 0.35 & 0.16 & 0.72 & 0.41 & & 0.28 & & & & 0.66 & 0.44 & 0.47 & 0.50 & 0.80 & & 0.52 & 0.52 \\
\hline-0.38 & 0.80 & 0.83 & 0.88 & 0.75 & 0.64 & 0.85 & 0.76 & 0.45 & 0.75 & 0.84 & 0.66 & & 0.78 & 0.82 & 0.87 & 0.87 & 0.87 & 0.84 & 0.87 & 0.79 \\
\hline-0.19 & 0.93 & 0.94 & 1.00 & 0.91 & 0.91 & 0.90 & 0.98 & 0.97 & 0.93 & 0.90 & 0.98 & 0.90 & 0.97 & 0.91 & 1.00 & 1.00 & 1.00 & 0.97 & 1.00 & 0.95 \\
\hline 0.00 & 1.00 & 1.00 & 0.91 & 1.00 & 1.00 & 1.00 & 1.00 & 1.00 & 0.92 & 1.00 & 1.00 & 1.00 & 1.00 & 0.93 & 0.89 & 0.76 & 0.82 & 1.00 & 0.86 & 0.95 \\
\hline 0.19 & 0.75 & 0.95 & 0.70 & 0.89 & 0.93 & 0.89 & 0.88 & 0.81 & 1.00 & 0.80 & 0.99 & 0.98 & 0.79 & 1.00 & 0.87 & 0.78 & 0.85 & 0.93 & 0.85 & 0.88 \\
\hline 0.38 & 0.65 & 0.92 & 0.61 & 0.86 & 0.89 & 0.70 & 0.68 & 0.88 & 0.98 & 0.98 & 0.78 & 0.92 & 0.71 & 0.92 & 0.85 & 0.83 & 0.75 & 0.87 & 0.76 & 0.82 \\
\hline 1.32 & 0.59 & 0.63 & 0.53 & 0.73 & 0.66 & 0.48 & 0.63 & 0.89 & 0.69 & 0.65 & 0.47 & 0.93 & 0.39 & 0.49 & 0.52 & 0.55 & 0.44 & 0.46 & 0.47 & 0.59 \\
\hline 1.51 & 0.51 & 0.61 & 0.62 & 0.57 & 0.69 & 0.48 & 0.56 & 0.91 & 0.66 & 0.48 & 0.55 & 0.95 & 0.34 & 0.40 & 0.44 & 0.41 & 0.37 & 0.50 & 0.59 & 0.56 \\
\hline 1.69 & 0.44 & 0.61 & 0.56 & 0.47 & 0.75 & 0.39 & 0.64 & 0.82 & 0.74 & 0.36 & 0.55 & 0.71 & 0.33 & 0.25 & 0.33 & 0.50 & 0.51 & 0.57 & 0.47 & 0.53 \\
\hline 1.88 & 0.42 & 0.68 & 0.49 & 0.43 & 0.70 & 0.35 & 0.74 & 0.61 & 0.63 & 0.47 & 0.51 & 0.54 & 0.33 & 0.21 & 0.26 & 0.54 & 0.51 & 0.75 & 0.29 & 0.50 \\
\hline 2.07 & 0.40 & 0.76 & 0.46 & 0.46 & 0.62 & 0.36 & 0.52 & 0.47 & 0.63 & 0.50 & 0.46 & 0.64 & 0.37 & 0.21 & 0.29 & 0.51 & 0.46 & 0.65 & 0.26 & 0.48 \\
\hline 2.26 & 0.48 & 0.57 & 0.46 & 0.45 & 0.55 & 0.40 & 0.41 & 0.42 & 0.54 & 0.47 & 0.44 & 0.70 & 0.47 & 0.21 & 0.33 & 0.50 & 0.37 & 0.63 & 0.34 & 0.46 \\
\hline
\end{tabular}


Appendix

Normalized fluorescence intensities of Spg singal measured for Figure 41C. - continuation

\begin{tabular}{|c|c|c|c|c|c|c|c|c|c|c|c|c|c|c|c|c|c|c|c|c|}
\hline \multirow{3}{*}{ 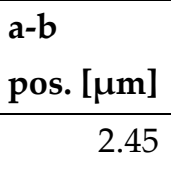 } & \multicolumn{20}{|c|}{ Normalized fluorescence intensities of Spg signal [a.u.] wild type } \\
\hline & \multicolumn{4}{|c|}{ Embryo 1} & \multicolumn{5}{|c|}{ Embryo 2} & \multicolumn{5}{|c|}{ Embryo 3} & \multicolumn{5}{|c|}{ Embryo 4} & \multirow{2}{*}{$\begin{array}{r}\text { Average } \\
0.45\end{array}$} \\
\hline & 0.45 & 0.45 & 0.54 & 0.45 & 0.45 & 0.41 & 0.46 & 0.51 & 0.59 & 0.49 & 0.65 & 0.68 & 0.34 & 0.22 & 0.30 & 0.33 & 0.34 & 0.43 & 0.43 & \\
\hline 2.64 & 0.35 & 0.37 & 0.58 & 0.43 & 0.42 & 0.35 & 0.49 & 0.56 & 0.55 & 0.41 & 0.60 & 0.74 & 0.31 & 0.21 & 0.30 & 0.31 & 0.28 & 0.47 & 0.39 & 0.43 \\
\hline 3.01 & 0.44 & 0.39 & 0.41 & 0.48 & 0.42 & 0.53 & 0.33 & 0.55 & 0.60 & 0.42 & 0.38 & 0.54 & 0.42 & 0.25 & 0.24 & 0.36 & 0.37 & 0.52 & 0.21 & 0.41 \\
\hline 3.20 & 0.48 & 0.38 & 0.37 & 0.43 & 0.44 & 0.60 & 0.31 & 0.53 & 0.50 & 0.40 & 0.34 & 0.40 & 0.39 & 0.18 & 0.27 & 0.45 & 0.44 & 0.50 & 0.29 & 0.41 \\
\hline 3.77 & 0.57 & 0.35 & 0.36 & 0.49 & 0.42 & 0.47 & 0.32 & 0.42 & 0.49 & 0.56 & 0.49 & 0.44 & 0.34 & 0.14 & 0.34 & 0.32 & 0.37 & 0.48 & 0.28 & 0.40 \\
\hline 3.95 & 0.64 & 0.26 & 0.29 & 0.51 & 0.56 & 0.41 & 0.34 & 0.42 & 0.32 & 0.63 & 0.35 & 0.62 & 0.39 & 0.15 & 0.45 & 0.32 & 0.58 & 0.37 & 0.23 & 0.41 \\
\hline 4.14 & 0.62 & 0.23 & 0.35 & 0.50 & 0.66 & 0.58 & 0.31 & 0.48 & 0.34 & 0.54 & 0.48 & 0.57 & 0.40 & 0.16 & 0.50 & 0.33 & 0.57 & 0.41 & 0.28 & 0.44 \\
\hline 4.33 & 0.58 & 0.27 & 0.33 & 0.44 & 0.58 & 0.49 & 0.31 & 0.40 & 0.44 & 0.39 & 0.43 & 0.49 & 0.39 & 0.20 & 0.39 & 0.35 & 0.39 & 0.40 & 0.30 & 0.40 \\
\hline 4.52 & 0.65 & 0.31 & 0.33 & 0.47 & 0.44 & 0.45 & 0.29 & 0.31 & 0.51 & 0.42 & 0.43 & 0.47 & 0.32 & 0.25 & 0.34 & 0.39 & 0.28 & 0.33 & 0.28 & 0.38 \\
\hline 5.46 & 0.69 & 0.28 & 0.60 & 0.56 & 0.42 & 0.60 & 0.40 & 0.45 & 0.55 & 0.61 & 0.49 & 0.38 & & 0.18 & 0.45 & & & 0.37 & & 0.47 \\
\hline 5.65 & 0.50 & 0.28 & 0.50 & 0.49 & 0.38 & & 0.31 & 0.34 & 0.45 & 0.73 & 0.50 & 0.52 & & 0.28 & 0.47 & & & 0.52 & & 0.45 \\
\hline 5.84 & 0.42 & 0.37 & 0.43 & 0.56 & 0.39 & & 0.33 & 0.46 & 0.56 & & 0.46 & 0.51 & & & 0.51 & & & 0.53 & & 0.46 \\
\hline 6.02 & 0.45 & 0.44 & & 0.57 & 0.40 & & & 0.55 & & & & 0.41 & & & 0.43 & & & 0.50 & & 0.47 \\
\hline 6.21 & & 0.37 & & 0.58 & 0.37 & & & 0.54 & & & & 0.58 & & & & & & & & 0.49 \\
\hline 6.40 & & 0.34 & & & 0.41 & & & 0.37 & & & & 0.54 & & & & & & & & 0.42 \\
\hline
\end{tabular}

$-192-$ 
Normalized fluorescence intensities of Spg singal measured for Figure 41C. - continuation

\begin{tabular}{|c|c|c|c|c|c|c|c|c|c|c|c|c|c|c|c|c|c|c|c|c|}
\hline 6.59 & & 0.42 & & & 0.45 & & & 0.48 & & & & 0.45 & & & & & & & & 0.45 \\
\hline \multicolumn{21}{|l|}{6.78} \\
\hline \multicolumn{21}{|l|}{6.97} \\
\hline $\begin{array}{l}\text { a-b } \\
\text { pos. }[\mu \mathrm{m}]\end{array}$ & \multicolumn{20}{|c|}{ Normalized fluorescence intensities of Spg signal [a.u.] dia } \\
\hline-1.69 & & & & 0.61 & & & & & & 0.53 & & & 0.62 & & & & 0.80 & & & 0.64 \\
\hline-1.51 & & & & 0.77 & & & & & & 0.63 & & & 0.70 & & & & 0.86 & & & 0.74 \\
\hline-0.94 & & 0.26 & 0.63 & 0.77 & 0.62 & 0.33 & & 0.54 & & 0.73 & 0.53 & & 0.80 & 0.48 & & & 0.74 & & & 0.59 \\
\hline-0.75 & 0.46 & 0.38 & 0.62 & 0.75 & 0.73 & 0.56 & & 0.72 & 0.40 & 0.73 & 0.67 & 0.64 & 0.85 & 0.71 & 0.78 & & 0.77 & & 0.42 & 0.64 \\
\hline-0.56 & 0.74 & 0.55 & 0.64 & 0.68 & 0.84 & 0.82 & 0.52 & 0.79 & 0.74 & 0.69 & 0.73 & 0.76 & 0.80 & 0.83 & 0.81 & 0.38 & 0.81 & 0.68 & 0.66 & 0.71 \\
\hline-0.38 & 0.83 & 0.70 & 0.78 & 0.76 & 0.87 & 0.88 & 0.74 & 0.90 & 0.88 & 0.71 & 0.83 & 0.89 & 0.82 & 0.83 & 0.88 & 0.69 & 0.81 & 0.84 & 0.84 & 0.81 \\
\hline-0.19 & 0.98 & 1.00 & 0.90 & 0.91 & 0.93 & 0.98 & 1.00 & 0.93 & 1.00 & 0.74 & 0.96 & 0.90 & 1.00 & 0.91 & 1.00 & 0.93 & 0.85 & 1.00 & 0.94 & 0.94 \\
\hline 0.75 & 1.00 & 0.71 & 0.81 & 0.93 & 0.80 & 0.87 & 0.76 & 0.87 & 0.84 & 0.77 & 0.66 & 0.90 & 0.72 & 0.80 & 0.80 & 0.63 & 0.88 & 0.74 & 0.99 & 0.81 \\
\hline 0.94 & 0.84 & 0.75 & 0.98 & 0.80 & 0.69 & 0.87 & 0.78 & 0.90 & 0.86 & 0.65 & 0.51 & 0.98 & 0.57 & 0.61 & 0.76 & 0.61 & 0.74 & 0.65 & 0.80 & 0.76 \\
\hline 1.13 & 0.69 & 0.78 & 0.86 & 0.67 & 0.68 & 0.74 & 0.80 & 0.86 & 0.81 & 0.54 & 0.58 & 0.78 & 0.55 & 0.64 & 0.76 & 0.54 & 0.86 & 0.69 & 0.65 & 0.71 \\
\hline 1.32 & 0.83 & 0.72 & 0.79 & 0.61 & 0.74 & 0.64 & 0.73 & 0.74 & 0.79 & 0.56 & 0.60 & 0.66 & 0.64 & 0.75 & 0.79 & 0.47 & 0.88 & 0.73 & 0.66 & 0.70 \\
\hline 1.51 & 0.61 & 0.65 & 0.90 & 0.63 & 0.78 & 0.59 & 0.69 & 0.67 & 0.71 & 0.69 & 0.55 & 0.77 & 0.55 & 0.70 & 0.54 & 0.47 & 0.73 & 0.71 & 0.70 & 0.66 \\
\hline 1.69 & 0.62 & 0.60 & 0.80 & 0.70 & 0.73 & 0.58 & 0.70 & 0.74 & 0.76 & 0.76 & 0.52 & 0.83 & 0.53 & 0.65 & 0.50 & 0.58 & 0.62 & 0.62 & 0.77 & 0.66 \\
\hline
\end{tabular}


Normalized fluorescence intensities of Spg singal measured for Figure 41C. - continuation

\begin{tabular}{|c|c|c|c|c|c|c|c|c|c|c|c|c|c|c|c|c|c|c|c|c|}
\hline \multirow{3}{*}{$\begin{array}{l}\begin{array}{l}\text { a-b } \\
\text { pos. }[\mu \mathrm{m}] \\
1.88\end{array}\end{array}$} & \multicolumn{20}{|c|}{ Normalized fluorescence intensities of Spg signal [a.u.] dia } \\
\hline & \multicolumn{4}{|c|}{ Embryo 1} & \multicolumn{5}{|c|}{ Embryo 2} & \multicolumn{5}{|c|}{ Embryo 3} & \multicolumn{5}{|c|}{ Embryo 4} & \multirow{2}{*}{$\begin{array}{r}\text { Average } \\
0.65\end{array}$} \\
\hline & 0.64 & 0.63 & 0.61 & 0.71 & 0.81 & 0.60 & 0.66 & 0.61 & 0.72 & 0.82 & 0.54 & 0.62 & 0.46 & 0.77 & 0.49 & 0.65 & 0.61 & 0.59 & 0.85 & \\
\hline 2.07 & 0.65 & 0.60 & 0.62 & 0.71 & 0.78 & 0.69 & 0.64 & 0.59 & 0.80 & 0.73 & 0.55 & 0.49 & 0.47 & 0.84 & 0.43 & 0.75 & 0.60 & 0.56 & 0.93 & 0.65 \\
\hline 2.45 & 0.93 & 0.53 & 0.54 & 0.62 & 0.59 & 0.71 & 0.54 & 0.55 & 0.91 & 0.70 & 0.49 & 0.53 & 0.62 & 0.90 & 0.59 & 0.57 & 0.43 & 0.66 & 0.82 & 0.64 \\
\hline 2.64 & 0.91 & 0.59 & 0.46 & 0.56 & 0.67 & 0.69 & 0.48 & 0.57 & 0.89 & 0.68 & 0.52 & 0.58 & 0.72 & 0.94 & 0.60 & 0.53 & 0.43 & 0.59 & 0.71 & 0.64 \\
\hline 3.20 & 0.55 & 0.77 & 0.54 & 0.61 & 0.78 & 0.71 & 0.57 & 0.66 & 0.70 & 0.45 & 0.40 & 0.67 & 0.50 & 0.89 & 0.66 & 0.41 & 0.60 & 0.50 & 0.68 & 0.61 \\
\hline 3.39 & 0.46 & 0.64 & 0.56 & 0.65 & 0.79 & 0.65 & 0.63 & 0.66 & 0.77 & 0.32 & 0.42 & 0.65 & 0.62 & 0.95 & 0.64 & 0.33 & 0.45 & 0.44 & 0.65 & 0.59 \\
\hline 3.58 & 0.53 & 0.70 & 0.59 & 0.57 & 0.79 & 0.71 & 0.62 & 0.72 & 0.73 & 0.29 & 0.55 & 0.66 & 0.79 & 0.82 & 0.55 & 0.31 & 0.43 & 0.53 & 0.67 & 0.61 \\
\hline 3.77 & 0.50 & 0.65 & 0.65 & 0.57 & 0.95 & 0.78 & 0.56 & 0.64 & 0.69 & 0.39 & 0.60 & 0.69 & 0.69 & 0.82 & 0.63 & 0.34 & 0.42 & 0.49 & 0.76 & 0.62 \\
\hline 3.95 & 0.58 & 0.50 & 0.80 & 0.58 & 0.93 & 0.79 & 0.52 & 0.58 & 0.66 & 0.75 & 0.58 & 0.50 & 0.67 & 0.69 & 0.62 & 0.47 & 0.46 & 0.47 & 0.67 & 0.62 \\
\hline 4.89 & 0.65 & 0.40 & 0.84 & & 0.76 & 0.71 & 0.51 & 0.39 & 0.74 & & 0.38 & 0.45 & & 0.41 & 0.46 & 0.71 & & 0.46 & 0.57 & 0.56 \\
\hline 5.08 & & 0.54 & 0.74 & & 0.68 & 0.68 & 0.52 & 0.45 & 0.74 & & 0.44 & 0.40 & & 0.52 & & & & 0.46 & 0.56 & 0.56 \\
\hline 5.27 & & 0.51 & 0.73 & & 0.60 & 0.70 & 0.58 & 0.54 & 0.66 & & 0.47 & 0.42 & & 0.59 & & & & 0.51 & 0.58 & 0.57 \\
\hline 5.46 & & & 0.77 & & 0.52 & 0.62 & 0.75 & 0.57 & 0.52 & & 0.58 & & & 0.63 & & & & 0.51 & 0.52 & 0.60 \\
\hline 5.65 & & & 0.60 & & 0.50 & 0.62 & 0.81 & 0.49 & 0.46 & & & & & 0.74 & & & & & 0.57 & 0.60 \\
\hline 5.84 & & & 0.57 & & 0.50 & 0.60 & 0.71 & 0.50 & & & & & & 0.70 & & & & & 0.61 & 0.60 \\
\hline
\end{tabular}

$-194-$ 
Normalized fluorescence intensities of Spg singal measured for Figure 41C. - continuation

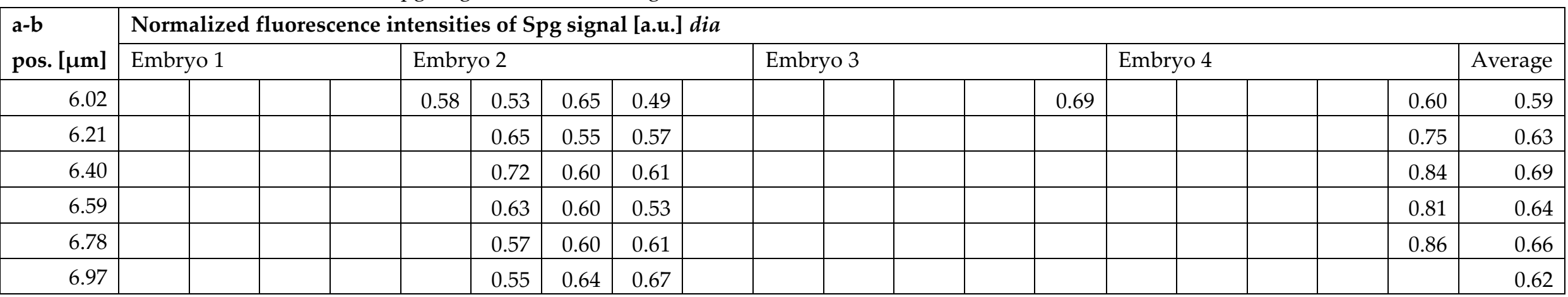


Table 16 Normalized fluorescence intensities of CnoYFP signal measured for Figure 45C.

\begin{tabular}{|c|c|c|c|c|c|c|c|c|}
\hline \multirow{2}{*}{$\begin{array}{l}\text { Time } \\
\text { [s] }\end{array}$} & \multicolumn{4}{|c|}{ Norm. fluorescence int. [a.u.] wild type } & \multicolumn{4}{|c|}{ Norm. fluorescence int. [a.u.] dia } \\
\hline & E1 & E2 & E3 & Average & E1 & E2 & E3 & Average \\
\hline-30.00 & 1.00 & 1.00 & 1.00 & 1.00 & 1.00 & 1.00 & 1.00 & 1.00 \\
\hline 0.00 & 0.13 & 0.12 & 0.18 & 0.14 & 0.16 & 0.14 & 0.16 & 0.15 \\
\hline 30.00 & 0.32 & 0.29 & 0.35 & 0.32 & 0.33 & 0.35 & 0.27 & 0.32 \\
\hline 60.00 & 0.35 & 0.44 & 0.49 & 0.43 & 0.52 & 0.48 & 0.44 & 0.48 \\
\hline 90.00 & 0.45 & 0.68 & 0.60 & 0.58 & 0.54 & 0.48 & 0.59 & 0.54 \\
\hline 120.00 & 0.54 & 0.68 & 0.78 & 0.67 & 0.70 & 0.70 & 0.71 & 0.70 \\
\hline 150.00 & 0.69 & 0.78 & 1.01 & 0.82 & 0.69 & 0.65 & 0.93 & 0.76 \\
\hline 180.00 & 0.72 & 0.77 & 0.95 & 0.81 & 0.77 & 0.78 & 0.94 & 0.83 \\
\hline 210.00 & 0.81 & 0.81 & 0.88 & 0.83 & 0.90 & 0.80 & 1.09 & 0.93 \\
\hline 240.00 & 0.85 & 0.67 & 0.89 & 0.80 & 0.76 & 0.70 & 1.22 & 0.89 \\
\hline 270.00 & 91 & 0.66 & 0.91 & 0.82 & 0.69 & 0.68 & 1.00 & 0.79 \\
\hline 300.00 & 0.94 & 0.74 & 0.94 & 0.87 & 0.80 & 0.77 & 1.17 & 0.91 \\
\hline 330.00 & 0.91 & 0.85 & 1.04 & 0.93 & 0.81 & 0.91 & 1.44 & 1.06 \\
\hline 360.00 & 90 & 0.89 & 1.04 & 0.94 & 0.75 & 0.88 & 1.51 & 1.05 \\
\hline 390.00 & 1.02 & 0.94 & 1.23 & 1.06 & 0.82 & 0.81 & 1.10 & 0.91 \\
\hline 420.00 & 1.00 & 0.86 & 1.10 & 0.99 & 0.95 & 0.59 & 1.05 & 0.86 \\
\hline 450.00 & 1.20 & 0.97 & 1.18 & 1.12 & 0.82 & 0.63 & 1.00 & 0.82 \\
\hline 480.00 & 1.12 & 0.99 & 1.01 & 1.04 & 0.82 & 0.72 & 1.09 & 0.88 \\
\hline 510.00 & 1.19 & 1.19 & 1.00 & 1.13 & 1.03 & 0.70 & 0.95 & 0.89 \\
\hline 540.00 & 1.12 & 1.14 & 1.10 & 1.12 & 0.98 & 0.67 & 1.07 & 0.91 \\
\hline 570.00 & 0.97 & 1.18 & 1.01 & 1.06 & 1.18 & 0.50 & 1.00 & 0.89 \\
\hline 600.00 & 0.91 & 1.15 & 1.16 & 1.07 & 1.10 & 0.71 & 1.24 & 1.02 \\
\hline 630.00 & 1.06 & 1.16 & 0.95 & 1.06 & 0.99 & 0.69 & 1.37 & 1.01 \\
\hline 660.00 & 1.03 & 1.03 & 1.02 & 1.02 & 1.00 & 0.81 & 1.31 & 1.04 \\
\hline 690.00 & 1.08 & 1.01 & 1.11 & 1.07 & 0.87 & 0.68 & 1.44 & 0.99 \\
\hline 720.00 & 1.05 & 0.95 & 1.14 & 1.05 & 0.87 & 0.67 & 1.56 & 1.03 \\
\hline 750.00 & 0.91 & 0.98 & 1.13 & 1.01 & 1.04 & 0.64 & 1.32 & 1.00 \\
\hline 780.00 & 0.99 & 0.99 & 1.16 & 1.05 & 1.09 & 0.60 & 1.43 & 1.04 \\
\hline 810.00 & 0.99 & 1.06 & 1.14 & 1.06 & 1.11 & 0.85 & 1.28 & 1.08 \\
\hline 840.00 & 1.02 & 1.04 & 1.15 & 1.07 & 0.87 & 0.87 & 1.47 & 1.07 \\
\hline
\end{tabular}

Table 17 Normalized fluorescence intensities of 117GFP signal measured for Figure 46B.

\begin{tabular}{|r|r|r|r|r|r|r|r|r|}
\hline \multirow{2}{*}{$\begin{array}{l}\text { Time } \\
\text { [s] }\end{array}$} & \multicolumn{7}{|c|}{ Normalized fluorescence intensity of 117GFP [a.u.] wild type } \\
\cline { 2 - 10 } & E1 & E2 & E3 & E4 & E5 & E6 & E7 & Average \\
\hline \hline-5.00 & 1.00 & 1.00 & 1.00 & 1.00 & 1.00 & 1.00 & 1.00 & 1.00 \\
\hline 0.00 & 0.15 & 0.16 & 0.17 & 0.14 & 0.11 & 0.12 & 0.11 & 0.13 \\
\hline 5.00 & 0.22 & 0.25 & 0.33 & 0.25 & 0.21 & 0.25 & 0.25 & 0.25 \\
\hline 10.00 & 0.31 & 0.35 & 0.42 & 0.38 & 0.28 & 0.35 & 0.36 & 0.35 \\
\hline 15.00 & 0.37 & 0.35 & 0.57 & 0.44 & 0.36 & 0.40 & 0.48 & 0.42 \\
\hline 20.00 & 0.38 & 0.39 & 0.61 & 0.54 & 0.40 & 0.43 & 0.51 & 0.47 \\
\hline 25.00 & 0.42 & 0.42 & 0.61 & 0.60 & 0.43 & 0.53 & 0.61 & 0.52 \\
\hline
\end{tabular}


Appendix

Normalized fluorescence intensities of 117GFP signal for Figure 46B - continuation

\begin{tabular}{|r|r|r|r|r|r|r|r|r|}
\hline 30.00 & 0.46 & 0.50 & 0.69 & 0.65 & 0.48 & 0.54 & 0.64 & 0.57 \\
\hline 35.00 & 0.51 & 0.47 & 0.76 & 0.66 & 0.55 & 0.60 & 0.78 & 0.62 \\
\hline 40.00 & 0.59 & 0.54 & 0.87 & 0.75 & 0.58 & 0.61 & 0.76 & 0.67 \\
\hline 45.00 & 0.55 & 0.51 & 0.84 & 0.73 & 0.61 & 0.65 & 0.87 & 0.68 \\
\hline 50.00 & 0.66 & 0.58 & 0.84 & 0.70 & 0.66 & 0.74 & 0.81 & 0.71 \\
\hline 55.00 & 0.67 & 0.55 & 0.93 & 0.71 & 0.68 & 0.61 & 0.89 & 0.72 \\
\hline 60.00 & 0.72 & 0.59 & 0.99 & 0.73 & 0.68 & 0.64 & 0.90 & 0.75 \\
\hline 65.00 & 0.79 & 0.59 & 1.05 & 0.68 & 0.76 & 0.67 & 0.87 & 0.77 \\
\hline 70.00 & 0.84 & 0.58 & 1.00 & 0.70 & 0.78 & 0.70 & 0.83 & 0.78 \\
\hline 75.00 & 0.82 & 0.57 & 1.14 & 0.65 & 0.94 & 0.71 & 0.82 & 0.81 \\
\hline 80.00 & 0.78 & 0.56 & 1.20 & 0.62 & 1.04 & 0.77 & 0.73 & 0.81 \\
\hline 85.00 & 0.85 & 0.60 & 1.21 & 0.84 & 1.04 & 0.79 & 0.68 & 0.86 \\
\hline 90.00 & 0.92 & 0.65 & 1.15 & 0.88 & 1.06 & 0.83 & 0.63 & 0.87 \\
\hline 95.00 & 0.83 & 0.65 & 1.20 & 0.91 & 1.04 & 0.89 & 0.66 & 0.88 \\
\hline 100.00 & 0.89 & 0.69 & 1.27 & 0.88 & 1.10 & 0.90 & 0.65 & 0.91 \\
\hline 105.00 & 0.95 & 0.67 & 1.06 & 0.94 & 1.15 & 0.84 & 0.70 & 0.90 \\
\hline 110.00 & 0.87 & 0.72 & 1.05 & 0.81 & 1.23 & 0.97 & 0.67 & 0.90 \\
\hline 115.00 & 0.94 & 0.65 & 0.99 & 0.80 & 1.25 & 0.89 & 0.66 & 0.88 \\
\hline & 0.96 & & 0.96 & & \\
\hline
\end{tabular}

\begin{tabular}{|c|c|c|c|c|c|c|c|}
\hline \multirow{2}{*}{$\begin{array}{l}\text { Time } \\
\text { [s] }\end{array}$} & \multicolumn{7}{|c|}{ Normalized fluorescence intensity of 117GFP [a.u.] dia } \\
\hline & E1 & E2 & E3 & E4 & E5 & E6 & Average \\
\hline-5.00 & 1.00 & 1.00 & 1.00 & 1.00 & 1.00 & 1.00 & 1.00 \\
\hline 0.00 & 0.12 & 0.26 & 0.15 & 0.10 & 0.16 & 0.11 & 0.15 \\
\hline 5.00 & 0.23 & 0.39 & 0.22 & 0.21 & 0.29 & 0.23 & 0.26 \\
\hline 10.00 & 0.30 & 0.52 & 0.26 & 0.30 & 0.46 & 0.27 & 0.35 \\
\hline 15.00 & 0.36 & 0.58 & 0.34 & 0.38 & 0.51 & 0.35 & 0.42 \\
\hline 20.00 & 0.48 & 0.66 & 0.39 & 0.46 & 0.59 & 0.44 & 0.50 \\
\hline 25.00 & 0.49 & 0.69 & 0.51 & 0.53 & 0.64 & 0.47 & 0.55 \\
\hline 30.00 & 0.54 & 0.67 & 0.56 & 0.58 & 0.67 & 0.49 & 0.59 \\
\hline 35.00 & 0.51 & 0.64 & 0.57 & 0.61 & 0.77 & 0.50 & 0.60 \\
\hline 40.00 & 0.61 & 0.69 & 0.62 & 0.70 & 0.76 & 0.49 & 0.64 \\
\hline 45.00 & 0.67 & 0.72 & 0.59 & 0.71 & 0.79 & 0.52 & 0.67 \\
\hline 50.00 & 0.67 & 0.80 & 0.61 & 0.78 & 0.72 & 0.60 & 0.70 \\
\hline 55.00 & 0.72 & 0.92 & 0.67 & 0.84 & 0.74 & 0.66 & 0.76 \\
\hline 60.00 & 0.79 & 0.96 & 0.69 & 0.81 & 0.78 & 0.70 & 0.79 \\
\hline 65.00 & 0.79 & 0.89 & 0.78 & 0.90 & 0.82 & 0.71 & 0.81 \\
\hline 70.00 & 0.73 & 1.00 & 0.81 & 0.99 & 0.80 & 0.67 & 0.83 \\
\hline 75.00 & 0.71 & 0.81 & 0.80 & 0.99 & 0.77 & 0.75 & 0.81 \\
\hline 80.00 & 0.77 & 0.84 & 0.85 & 0.88 & 0.68 & 1.00 & 0.84 \\
\hline 85.00 & 0.79 & 0.89 & 0.92 & 0.96 & 0.73 & 1.00 & 0.88 \\
\hline 90.00 & 0.87 & 0.91 & 0.84 & 0.86 & 0.76 & 1.03 & 0.88 \\
\hline 95.00 & 0.91 & 0.91 & 0.84 & 0.76 & 0.71 & 1.22 & 0.89 \\
\hline 100.00 & 0.93 & 0.93 & 0.86 & 0.74 & 0.67 & 1.24 & 0.89 \\
\hline 105.00 & 0.82 & 0.92 & 0.84 & 0.64 & 0.72 & 1.54 & 0.91 \\
\hline 110.00 & 0.70 & 0.86 & 0.76 & 0.68 & 0.75 & 1.48 & 0.87 \\
\hline
\end{tabular}


Normalized fluorescence intensities of 117GFP signal for Figure 46B - continuation

\begin{tabular}{|l|l|l|l|l|l|l|l|l|}
\hline 115.00 & 0.78 & 0.93 & 0.77 & 0.75 & 0.73 & 1.40 & & 0.89 \\
\hline
\end{tabular}

Table 18 Averages of normalized fluorescence intensities per embryo measured for Figure 54B.

\begin{tabular}{|c|c|c|c|c|c|c|}
\hline \multirow{2}{*}{$\begin{array}{l}\text { Position } \\
{[\mu \mathrm{m}]}\end{array}$} & \multicolumn{6}{|c|}{ Normalized fluorescence intensities averaged per embryo [a.u.] } \\
\hline & WT 1 & WT 2 & WT 3 & dia 1 & dia 2 & dia 3 \\
\hline-2.90 & 0.58 & 0.47 & 0.43 & 0.63 & 0.58 & 0.52 \\
\hline-2.77 & 0.56 & 0.48 & 0.44 & 0.64 & 0.55 & 0.51 \\
\hline-2.64 & 0.57 & 0.49 & 0.44 & 0.63 & 0.55 & 0.52 \\
\hline-2.50 & 0.56 & 0.50 & 0.45 & 0.62 & 0.57 & 0.52 \\
\hline-2.37 & 0.57 & 0.51 & 0.44 & 0.62 & 0.59 & 0.53 \\
\hline-2.24 & 0.58 & 0.51 & 0.45 & 0.62 & 0.59 & 0.53 \\
\hline-2.11 & 0.58 & 0.52 & 0.45 & 0.63 & 0.60 & 0.54 \\
\hline-1.98 & 0.57 & 0.54 & 0.44 & 0.64 & 0.60 & 0.55 \\
\hline-1.85 & 0.57 & 0.54 & 0.45 & 0.65 & 0.60 & 0.55 \\
\hline-1.71 & 0.58 & 0.54 & 0.46 & 0.66 & 0.61 & 0.57 \\
\hline-1.58 & 0.59 & 0.53 & 0.46 & 0.67 & 0.62 & 0.60 \\
\hline-1.45 & 0.60 & 0.53 & 0.47 & 0.68 & 0.63 & 0.62 \\
\hline-1.32 & 0.61 & 0.53 & 0.48 & 0.71 & 0.65 & 0.65 \\
\hline-1.19 & 0.62 & 0.53 & 0.50 & 0.72 & 0.68 & 0.67 \\
\hline-1.05 & 0.63 & 0.55 & 0.52 & 0.74 & 0.71 & 0.69 \\
\hline-0.92 & 0.63 & 0.57 & 0.55 & 0.76 & 0.74 & 0.72 \\
\hline-0.79 & 0.65 & 0.59 & 0.56 & 0.79 & 0.78 & 0.76 \\
\hline-0.66 & 0.68 & 0.63 & 0.59 & 0.81 & 0.81 & 0.79 \\
\hline-0.53 & 0.71 & 0.67 & 0.62 & 0.84 & 0.84 & 0.82 \\
\hline-0.40 & 0.74 & 0.72 & 0.69 & 0.87 & 0.88 & 0.86 \\
\hline-0.26 & 0.82 & 0.81 & 0.79 & 0.91 & 0.93 & 0.91 \\
\hline-0.13 & 0.93 & 0.93 & 0.93 & 0.97 & 0.98 & 0.97 \\
\hline 0.00 & 1.00 & 1.00 & 1.00 & 1.00 & 1.00 & 1.00 \\
\hline 0.13 & 0.95 & 0.93 & 0.91 & 0.97 & 0.97 & 0.97 \\
\hline 0.26 & 0.83 & 0.83 & 0.74 & 0.91 & 0.93 & 0.93 \\
\hline 0.40 & 0.74 & 0.74 & 0.64 & 0.88 & 0.88 & 0.88 \\
\hline 0.53 & 0.69 & 0.67 & 0.59 & 0.85 & 0.85 & 0.84 \\
\hline 0.66 & 0.66 & 0.63 & 0.56 & 0.83 & 0.82 & 0.80 \\
\hline 0.79 & 0.63 & 0.60 & 0.54 & 0.80 & 0.78 & 0.77 \\
\hline 0.92 & 0.62 & 0.59 & 0.52 & 0.76 & 0.74 & 0.74 \\
\hline 1.05 & 0.60 & 0.57 & 0.51 & 0.74 & 0.71 & 0.71 \\
\hline 1.19 & 0.58 & 0.55 & 0.49 & 0.74 & 0.69 & 0.69 \\
\hline 1.32 & 0.58 & 0.53 & 0.48 & 0.73 & 0.67 & 0.67 \\
\hline 1.45 & 0.57 & 0.53 & 0.47 & 0.70 & 0.65 & 0.65 \\
\hline 1.58 & 0.55 & 0.53 & 0.47 & 0.68 & 0.62 & 0.63 \\
\hline 1.71 & 0.55 & 0.54 & 0.47 & 0.66 & 0.60 & 0.62 \\
\hline 1.85 & 0.56 & 0.55 & 0.48 & 0.66 & 0.59 & 0.60 \\
\hline 1.98 & 0.56 & 0.55 & 0.48 & 0.65 & 0.59 & 0.60 \\
\hline
\end{tabular}


Averages of normalized fluorescence intensities per embryo measured for Figure 54B. - continuation

\begin{tabular}{|l|l|l|l|l|l|l|}
\hline 2.11 & 0.55 & 0.53 & 0.48 & 0.64 & 0.58 & 0.59 \\
\hline 2.24 & 0.54 & 0.52 & 0.48 & 0.64 & 0.57 & 0.58 \\
\hline 2.37 & 0.55 & 0.51 & 0.47 & 0.63 & 0.57 & 0.56 \\
\hline 2.50 & 0.57 & 0.52 & 0.46 & 0.64 & 0.57 & 0.55 \\
\hline 2.64 & 0.57 & 0.53 & 0.47 & 0.63 & 0.55 & 0.52 \\
\hline 2.77 & 0.57 & 0.52 & 0.48 & 0.61 & 0.54 & 0.51 \\
\hline 2.90 & 0.54 & 0.49 & 0.47 & 0.59 & 0.54 & 0.52 \\
\hline 3.03 & 0.52 & 0.52 & 0.48 & 0.59 & 0.54 & 0.52 \\
\hline
\end{tabular}

Table 19 Width of Cpa signal curves measured for Figure 54C.

\begin{tabular}{|c|c|c|c|c|c|}
\hline \multicolumn{6}{|c|}{ Width of fluorescence intensity curves of Cpa signal at $80 \%$ signal intensity $[\mu \mathrm{m}]$} \\
\hline \multicolumn{3}{|l|}{ Wild type } & \multicolumn{3}{|l|}{ dia } \\
\hline Embryo 1 & Embryo 2 & Embryo 3 & Embryo 1 & Embryo 2 & Embryo 3 \\
\hline 0.65 & 0.96 & 0.75 & 2.31 & 1.56 & 1.82 \\
\hline 0.77 & 0.70 & 0.46 & 2.32 & 1.84 & 1.43 \\
\hline 0.63 & 0.48 & 0.48 & 1.57 & 1.80 & 1.95 \\
\hline 0.55 & 0.53 & 0.55 & 1.35 & 1.19 & 1.59 \\
\hline 0.70 & 0.45 & 0.31 & 0.92 & 0.92 & 1.46 \\
\hline 0.74 & 1.27 & 0.67 & 1.36 & 1.74 & 1.07 \\
\hline 0.60 & 0.95 & 0.67 & 1.26 & 1.61 & 1.27 \\
\hline 0.58 & 0.71 & 0.41 & 2.48 & 1.40 & 1.57 \\
\hline 0.54 & 0.48 & 0.50 & 1.69 & 0.94 & 0.94 \\
\hline 0.37 & 0.51 & 0.39 & 2.03 & 1.14 & 0.81 \\
\hline 0.91 & 1.13 & 0.43 & 2.15 & 0.86 & 1.49 \\
\hline 1.02 & 0.63 & 0.52 & 1.58 & 1.69 & 1.05 \\
\hline 0.65 & 0.90 & 0.40 & 1.27 & 1.46 & 1.86 \\
\hline 0.81 & 1.04 & 0.39 & 1.44 & 1.85 & 1.25 \\
\hline 1.00 & 0.97 & 0.47 & 1.86 & 2.00 & 0.86 \\
\hline 0.82 & 1.16 & 0.56 & 2.23 & 1.71 & 1.44 \\
\hline 0.81 & 0.86 & 0.38 & 2.27 & 1.87 & 1.48 \\
\hline 0.87 & 0.49 & 0.46 & 1.48 & 1.75 & 1.12 \\
\hline 0.56 & 0.58 & 0.41 & 2.25 & 0.68 & 1.97 \\
\hline 0.33 & 0.71 & 0.35 & 1.20 & 1.13 & 1.16 \\
\hline 1.37 & 0.83 & 0.68 & 1.81 & 2.33 & 1.12 \\
\hline 1.03 & & 0.48 & 1.06 & 1.71 & 1.81 \\
\hline 0.79 & & 0.46 & 1.71 & 1.20 & 0.88 \\
\hline 0.45 & & 0.48 & 1.13 & 1.45 & 1.01 \\
\hline 0.78 & & 0.55 & 0.86 & 1.78 & 1.69 \\
\hline 0.56 & & 0.52 & 1.57 & 1.43 & 1.83 \\
\hline 0.94 & & 0.60 & 1.81 & 1.27 & 1.32 \\
\hline 0.91 & & 0.76 & 1.52 & 1.09 & 1.28 \\
\hline 0.60 & & 0.48 & 0.90 & 1.19 & 1.26 \\
\hline 0.53 & & 0.40 & 1.10 & 1.41 & 1.71 \\
\hline
\end{tabular}


Width of Cpa signal curves measured for Figure 54C. - continuation

\begin{tabular}{|r|r|r|r|r|r|}
\hline 0.48 & & 0.88 & 2.13 & 1.75 & 1.26 \\
\hline 0.90 & & 1.10 & 1.77 & 2.11 & 1.67 \\
\hline 0.69 & 1.03 & 2.09 & 1.57 & 1.33 \\
\hline 0.52 & 0.93 & 1.10 & 1.41 & 1.11 \\
\hline 0.43 & 0.90 & 2.01 & 1.62 & 1.08 \\
\hline 0.48 & 0.51 & & 1.43 & 0.93 \\
\hline 0.35 & 0.45 & & & 1.54 \\
\hline 0.46 & 0.37 & & & \\
\hline 1.08 & & & & & \\
\hline
\end{tabular}

Table 20 Exponential decay of Cpa curves fitted for Figure 55C.

\begin{tabular}{|r|r|}
\hline \multicolumn{2}{|l|}{ Exponential decay [a.u.] } \\
\hline WT & dia \\
\hline \hline 0.59 & 1.19 \\
\hline 0.54 & 1.46 \\
\hline 0.44 & 1.15 \\
\hline 0.51 & 1.31 \\
\hline 0.39 & 1.68 \\
\hline 0.62 & 1.34 \\
\hline Average \\
\hline \multicolumn{2}{|c|}{} \\
\hline 0.51 & 1.36 \\
\hline
\end{tabular}

Table 21 Fluorescence intensities of Phalloidin measured for Figure 56C.

\begin{tabular}{|r|r|}
\hline \multicolumn{2}{|l|}{ Fluorescence intensity [a.u.] } \\
\hline WT & dia \\
\hline \hline 1528.40 & 1596.08 \\
\hline 1446.49 & 1436.46 \\
\hline 1521.25 & 1654.39 \\
\hline 1481.74 & 1667.82 \\
\hline 1945.86 & 1870.67 \\
\hline 1821.08 & 1626.99 \\
\hline 1996.18 & 1251.53 \\
\hline 1782.33 & 1324.88 \\
\hline 1370.34 & 1244.74 \\
\hline 1341.67 & 1238.17 \\
\hline 1278.47 & 1352.77 \\
\hline 1473.23 & 1280.93 \\
\hline \multicolumn{2}{|c|}{ Average } \\
\hline 1582.25 & 1462.12 \\
\hline
\end{tabular}


Table 22 Distance of flies measured in negative geotaxis assay for Figure 64 .

\begin{tabular}{|r|l|r|r|r|r|r|r|}
\hline & & \multicolumn{3}{|c|}{ P/ TM3 Distance [cm] } & \multicolumn{3}{|c|}{ P/ Def Distance [cm] } \\
\hline$\#$ & sex & $\mathbf{0 : 3 0} \mathbf{~ m i n}$ & $\mathbf{1 : 0 0} \mathbf{~ m i n}$ & $\mathbf{1 : 3 0} \mathbf{~ m i n}$ & $\mathbf{0 : 3 0} \mathbf{~ m i n}$ & $\mathbf{1 : 0 0} \mathbf{~ m i n}$ & $\mathbf{1 : 3 0} \mathbf{~ m i n}$ \\
\hline \hline 1 & $\mathrm{m}$ & 19,00 & 19,00 & 19,00 & 0,00 & 0,00 & 0,00 \\
\hline 2 & $\mathrm{~m}$ & 0,00 & 0,00 & 0,00 & 0,00 & 0,00 & 0,00 \\
\hline 3 & $\mathrm{~m}$ & 16,00 & 19,00 & 19,00 & 0,00 & 0,00 & 0,00 \\
\hline 4 & $\mathrm{~m}$ & 19,00 & 19,00 & 19,00 & 0,00 & 0,00 & 0,00 \\
\hline 5 & $\mathrm{~m}$ & 13,00 & 17,00 & 19,00 & 0,00 & 0,00 & 0,00 \\
\hline 6 & $\mathrm{~m}$ & 6,50 & 8,00 & 5,00 & 4,00 & 4,00 & 6,00 \\
\hline 7 & $\mathrm{~m}$ & 15,00 & 16,00 & 18,00 & 0,00 & 0,00 & 0,00 \\
\hline 8 & $\mathrm{f}$ & 19,00 & 19,00 & 19,00 & 0,00 & 0,00 & 2,00 \\
\hline 9 & $\mathrm{f}$ & 17,00 & 19,00 & 19,00 & 0,00 & 0,00 & 0,00 \\
\hline 10 & $\mathrm{f}$ & 17,00 & 2,00 & 13,00 & 0,00 & 0,00 & 0,00 \\
\hline 11 & $\mathrm{f}$ & 15,00 & 19,00 & 19,00 & 2,00 & 2,00 & 2,00 \\
\hline 12 & $\mathrm{f}$ & 19,00 & 19,00 & 19,00 & & & \\
\hline \multicolumn{2}{|c|}{ Average } & 14,63 & 14,67 & 15,67 & 0,55 & 0,55 & 0,91 \\
\hline
\end{tabular}

